\title{
Mapping of electrically induced atrial fibrillation in humans
}

Citation for published version (APA):

Konings, K. T. S. (1999). Mapping of electrically induced atrial fibrillation in humans. [Doctoral Thesis, Maastricht University]. Universiteit Maastricht. https://doi.org/10.26481/dis.19990122kk

Document status and date:

Published: 01/01/1999

DOI:

10.26481/dis.19990122kk

Document Version:

Publisher's PDF, also known as Version of record

\section{Please check the document version of this publication:}

- A submitted manuscript is the version of the article upon submission and before peer-review. There can be important differences between the submitted version and the official published version of record.

People interested in the research are advised to contact the author for the final version of the publication, or visit the DOI to the publisher's website.

- The final author version and the galley proof are versions of the publication after peer review.

- The final published version features the final layout of the paper including the volume, issue and page numbers.

Link to publication

\footnotetext{
General rights rights.

- You may freely distribute the URL identifying the publication in the public portal. please follow below link for the End User Agreement:

www.umlib.nl/taverne-license

Take down policy

If you believe that this document breaches copyright please contact us at:

repository@maastrichtuniversity.nl

providing details and we will investigate your claim.
}

Copyright and moral rights for the publications made accessible in the public portal are retained by the authors and/or other copyright owners and it is a condition of accessing publications that users recognise and abide by the legal requirements associated with these

- Users may download and print one copy of any publication from the public portal for the purpose of private study or research.

- You may not further distribute the material or use it for any profit-making activity or commercial gain

If the publication is distributed under the terms of Article $25 \mathrm{fa}$ of the Dutch Copyright Act, indicated by the "Taverne" license above, 
| Mapping of EleCtracally induced atrial fHBRILLATION IN HUMANS | 


\section{Conorow}

01999 K.T.S. Konings, Mastricht

$430100-5278-244 n$

Vormgeving: Milianthe Wouters, Lenting en Terlingen Grafsch Onwerpers BNO, Matstricht Drak: Datawyse I Uniwersitaire Pers Maastricht 


\section{Mapping of electrically induced atrial

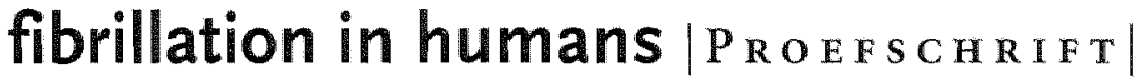

ter verkriging van de graad wan doctor aan de Universiteit Mastricht, op gezag wan de Rector Magnificus, Prof. Dr. A. Nieuwenhujizen Kruseman, volgens het besluit wan het College van Decanen, in het openbaar te verdedigen op vrijdag, 22 januari 1999 on 14.00 uur

dixan

Karen Theodore Susan Konings geboren op 12 Juni 1967 te Groningen

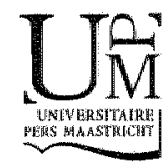


Fronorom:

Prof. Dr. M.A.Allessie

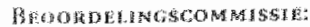

Prof. Dr. H.J.). Wellens (Voorzitter)

Prof. Dr. H.. J.G.M. Criljns (Rjjksuniversiteit Groningen)

Prof. Dr. H.J. Jongsma (Rijkstmiversiteit Utrecht)

Prof. Dr. R.S. Reneman

Dr. I.I. R.M. Smeets

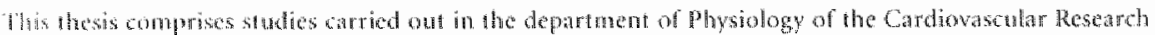

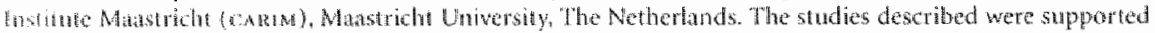

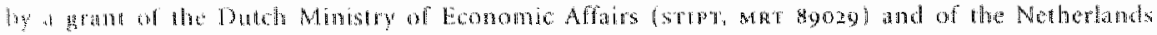

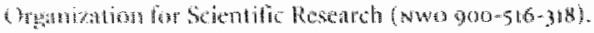

Financial support supplied by the Netherlands Heart Foundation for the publication of this thesis is graterully acknowledged.

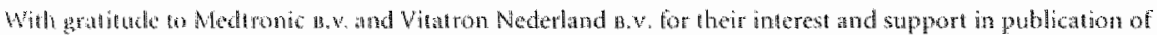
this thesist. 
"It is a mistake to believe that a science consists in nothing but exclusively proved propositions, and it is tanjust to demand that it should."

"Hypothesis has its right place, it forms a working basis; but it is an acknowledged whakeshifi, and as a fittal expression of opinion, it is an open confession either of fallute or at the best of purpose uninacounplished."

Sir Thonas L.chin 


$$
\text { , }
$$




\section{CHAPTER}

General Introduction

Historical Remarks

Sir Thomas Lenwis

Gordon K Moe

Chapter 2 |

High-Density Mapping of Electrically Induced Atrial Fibrillation in Humans

$\mid$ Chapter $3 \mid$

Configuration of Unipolar Atrial Electrograms during Electrically Induced Atrial

Fibrillation in Humans

\section{CHAPTER 4}

Mapping of the Right Atrium during Acute and Chronic Atrial Fibrillation in the Goat

\section{CHAPTER 5}

General Discussion

Mechanisms of Atrial ribrillation

Mapping of Aarial Fibrillation

ffathoyphysiology of atrial Fibrillation

Therapeatical Optons

\section{APPENDIX}

Summary

Samenvatting.

Nawoord

Curriculum Vitae 



\section{Chapter 1 | General Introduction |}

"The history of the recognition of fibrillation of the auricles will impress you

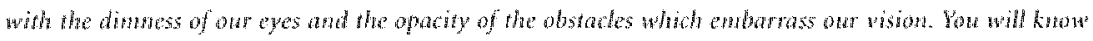

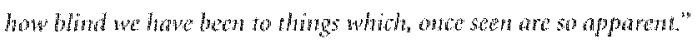

Sir Thomas Lewis' 
Almost 150 years ago fibrillation in the ventricles was first described by Hoffa and Ludwig. ${ }^{2}$ At that time it was considered to be an experimental condition with no clinical rellevance. However, 37 years later a condition in the human heart with similar characteristics was described by McWilliam ${ }^{3}$ in patients having a "conspicuously irregular arterial pulse", which was attributed to "delirimm of the heart" "On examination these patients suffered from palpitations, exercise intolerance, easy exhaustion, and a general feeling of ill-being. The radial pulse revealed a totally disordered rhythm; heart action being highly rapid and irregullar, strong pulsations taking turns with runs of amost imperceptible beats, and the interwal between beats varying markedly. 1,4 The heart rate varied greatly from case to case (30-220 beats per minute), although a fast pulse, approximately double the nomal rate of the ventricle, was the rule. Comparison of venous pulse curves (representing atrial contractions) with anterial pulse curves (ventricular contractions) showed, that there was no clear relation between the activity of atria and ventricles. A variable number of small atrial contractions occurred during each wentricular cycle (Figure 1.1). Notwithstanding these striking symptoms, it took until 19a. before the conspicuous irregularity was considered to be a specific arrhythmia." This cardiac arrhythmia was given many different names: pulsus arrhythmicus, pulsus itregularis perpetuus," paralysis of the auricle, nodal rhythm, irregular tachycardia, chronic arrhythmia, arrhythmia perpetua, and auricular fibrillation. It was characterized in a classic but obsolete nomenclature with the adjectives "inaequalis", "deficiens" and "intermittens". Clearly, this irregular heart beat caused confusion. Now we know that all these terms were attempts to describe atrial fibrillation; the changing terminology being the result of new observations and better understanding. Only after the galvanometric exanination of the heart became possible, the atral nature of this irregular heart thythm became evident.

Athough by that time it was already known that cardiac contractions are caused by electrical impulses, "i the electrophysiology of the heart could not be properly investigated until 1903, when Einthoven"s invented the galvanometer as a tool to recond the electrical activity of the heart. In a normal electrocandiogram (EcG) (Figure 1.2, upper panel) the ventricular depolarization is visualized by a large and narrow deflection from bastine, named the ars-complox, after which ventricular repolarization is represented by a wide hump, mamed the Twwave. Atrial activity, 


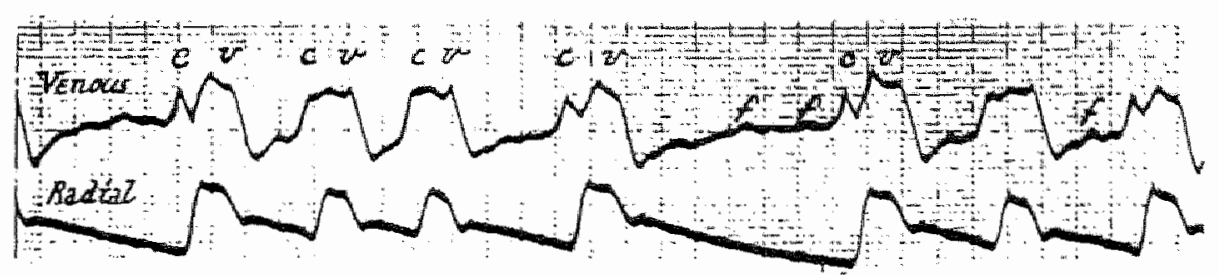

Simultaneous venous and radial curves from a case of atrial fibrillation. The venous curve shows the undulations $f, f$, in the longest diastoles. The radiall curve displays severe irregularity of the pulse. From Lewis: Chapter 23 . Figure 272.

which in normal conditions begins and ends shortly before the ventricular depolarization, inscribes a small mound ( $p$-wave) on the electrocatdiogram. During atrial fibrillation, this normal regular pattern is totally disturbed (Figure 1.2 , lower panel). The first clinical electrocardiogram displaying atrial fibrillation was published in 1906. ${ }^{16}$ However, it was not until Rothberger and Winterbergly and Lewis ${ }^{3}$ published a series of such ecg tracings that uniforn criteria for the diagnosis of atrial fibrillation were formulated: total irregularity of the ventricular cycles ( $R-R$-intervals), absence of discrete atrial activation ( $\mathrm{P}$-waves), and irregular fast oscillations of the baseline.

With the galvanometer, Sir Thomas Lewis performed a series of elegant experiments on the mechanism and graphic registration of the heart-beat $(9904-1925)$. In an unparalleled eloquent style he deliberated upon the mechanisms of arrhythmias, and gave way to the important "Multiple wavelet hypothesis" of Gordon K Moe. ${ }^{18}$ Sir Thonas Lewis and Gordon K Moe did their investigations over 90 and 30 years ago with, in our wiew, quite sinple methods. Nonetheless, they created major breakthroughs in our understanding of the mechanisms of atrial fibrillation. Since the work of these wo early researchers provided the basis for the modem studics presented in this thesis, their research on the mechanisms of at rial fibrillation will first be reviewed in some detail.

SHETHOMAS LEREYS

The absence of any electrical sign of organized atrial activity ( $p$-waves) and the apparent standstill of the atria when inspected in vivo, originally led to the idea that during "complete irregularity of the heart" the atria were paralyzed," and 
that the ventricular thythm originated in the aw-nodes or the ventricles themselvers. Later Lewis and Mackenzie assumed that the atria were actually active, because at autopsy they were found to be hypertrophied, and because cases were observed in which a normal rhythm reasserted itself." A paper of Cushny and Edmunds, 20 in which the similarity was described between arterial curves of atrial fibrillation under experimental conditions and in patients wrth complete irregularity of the heart, drew Lewis's attention to the arrhythmia. Careful examination of $\mathrm{ECG}$-recordings during atrial fibrillation, led to the idea that the origin of the wentricular contraction during 'complete cardiac irregularity" lies in the atriat; the shape of the wentricular complexes (QRS and $T$-waves) are the same during sinus rhythm or atrial fibrillation, showing that in the ventricles the excitation wave spreads allong nomal paths (Figure 1.2), Lewis observed that the p-waves were replaced by oscillations of greater or lesser amplitude. These "peculiar and distinctive" oscillations were variable from case to case in prominence, shape, and frequency (400-600 beats per minute). Often they were less marked, appearing only occasionally (Figure 1.2). Such oscillations were also observed in venous pulse curves, but were erroneously interpreted as being caused by tremor of the body muscles. Nevertheless, they were always found during atrial fubrillation, and not under other conditions. The atrial source of the arrhythmia was demonstrated by precordial ecg leads. At the portion of the chest overlying the atria, the amplitude of the rapid irregular oscillations was maximal, whereas at ventricular sites this was less prominent. Their persistence throughout the candiac cycle showed that the atrial activity was continuous. Altogether these results pointed to an uncoordinated but continuous electrical activity in the atria during "complete cardiac irregularity"? Howewer, at that time this view was not generally accepted and the opinion prevailed that inegularity of the heart was a result of distention of its walls. It was inconceivable to those "obsessed" by a purely mechanical doctrine of heart disease, that the insignificant atrial function could be so profoundly and persistently disturbed, so as to result in a disorder that could continue for many years.

Lewis attempted to obtain further ewidence "that would silence all criticism", and therefore turned to animal studies, hoping to discover atrial tibrillation as a manifestation of a disease in them." In camine experiments he induced an atrial rhythm, that lagely resembled the clinical syndrom by application of faradic current. This experimental arrhythmia was already known as atrial fibrillation, and always prowoked an outspoken ventricular irregularity. The ventricular 

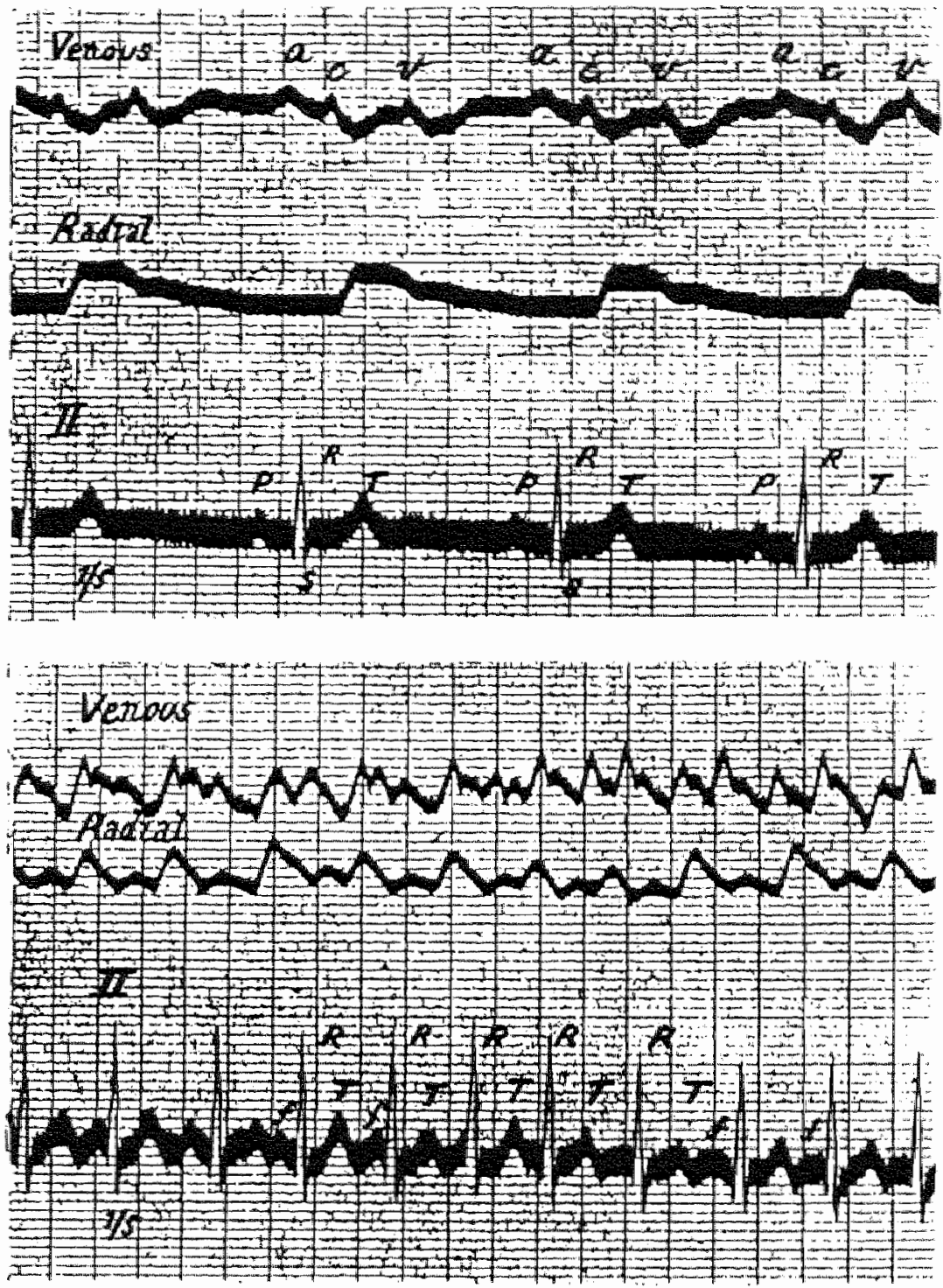

Simultaneous venous, padial and electrocardiographic curves from a patient with atrial fibrillation. In a period of sinus rhythm (upper panel), the heart action is regular and slow, the Figure 1.2 wenous and electrocardiographic curves demonstrating a perfectly normal mechanism. During the attacks of atriall fibrillation (lower panell), the ventricle beats fast and irregular. The venous curve shows fine and course undulations. In the electracardiogram the characteristic features of atriall fibrillation are visible; The R-R interval is totally irregular and there are no p-waves. In this case also fine oscillations $(f, f)$ are wisible in the short diastoles that often fall within the $T$ wave and distort them. From Lewis ${ }^{\prime \prime}$ ' Chapter 23 , Figures 275 and 276. 
irregularty and incoordination betwen atria and ventricles used to be interpreted as the result of a partial atrioventricular block due to the fast atrial rate. By comparing ECG $\$$, epicardial etectrograms, and radial and venous curves, he could prove that the clinical disorder and the experimentally induced arrhythmia were one and the same. The ventricular complexes were similar in shape and irregularity, and the atrial rate was equally tapid. The unavoidable conclusion was that complete irregularity of the heart indeed was the result of atrial fibrilation. ${ }^{7}$ In his experimental model, Lewis also found further proof for atrial activity during atrial fibrilation. When atrial contraction was measured with a myocardiographic lever, no coordinate atrial activity could be demonstrated. However, on closer inspection, the muscle appeared to be broken up in to many small areas, each of which seemed to be contracting energetically, with no relation to the contraction in surrounding areas. Lewis compared it to a surface of water disturbed by a downpour of ram" (while during sinus rhythm the electrical impulse propagates in a radial fashion from the sinus node, as the spread of fluid poured upon an almost flat surface ${ }^{22}$ ).

He showed that, although atrial fibrillation was often associated with orher cardiac diseases such as mitral stenosis, ventricular dilatation and infarction, it was not necessarily linked to structural changes in the heart. He noted that atrial fibrillation was absent in many cases of severe myocandial disease, while a patient without any myocardial disease or dilatation, could show perfect fibrillatory undulations. Furthermore, fibrillation of the atria could be paroxysmal, and he did not think it possible to distinguish between tissue taken from hearts during attacks or during periods of sinus rhythm. "Therefore, he concluded that atrial fibrillation is a functional rather than a structural disorder. ${ }^{7}$

\section{Ectopic Foci?}

It remained unsolwed what causes of atrial fibrillation. Lewis supported the view that dibrillation is caused by numerous electrical impulses in the atriall walls. Initially. he regarded it as a state in which stimuli are generated at many separate points. The incoordination of the contracting fibers then will be the result of multiple umpulses that block locally. However, a satisfactory proof was not given since the known causes of to new impulses, such as increased cardiac pressure, fibrosis, and ischemia bore no constant relationship to the arthythmia. For the time being, Lewis reganded atrial fibrillation as extrasystolic in nature, probably on the basis of abnormal impulse formation. "Supposing that two or more ectopic impulses 
are started at the same time at different points in the musculature of an irritable atrium, are not those factors present which are calculated to produce a condition of absolute incoordination? It is possible that fibrillation, when once produced under suitable circumstances, may itself maintain the increased irritability, and thereby tend to the continuation of the incoondinate state." "Whe concept that atrial fibrillation is due to multiple ectopic foci was deriwed from the observations made in wentricular fibrillation by Engelmann ${ }^{23}$ and Winterberg. ${ }^{24}$ Further investigation was temporarily postponed by the first word war. ${ }^{25}$ Only later Lewis realized that another possible mechanism had to be taken into account: an excitation wave may pass more than once through the same tissue, and thus two or more beats may result from a single circulating impulse.

\section{Theory of Circus Movement}

The first study of circus movement was performed by Mayer in the bell of jelly fish, ${ }^{26}, 27$ and continued by Mines in ring preparations cut from the atria and ventricles of large rays, turtles ${ }^{25}$ and dog ventricles. ${ }^{30}$ Mines showed that under certain conditions, local stimulation can start a wave in one direction. In the other direction a local block can occur, for instance by increasing the rate of stimulation." Such a wave can run around the ring so slowly that the refiactory phase has ended in each part of the ring when the wave approaches it. Thus, the wave circulates and may continue to do so infinitely (Figure 1.3). The rate of beating is then controlled by three factors: the conduction velocity and the duration of the refractory period on one hand, and the size of the circuit on the other. ${ }^{30}$ At the same time, Garrey ${ }^{32}$ produced circus contractions in turtle wentricle, for which unidirectional conduction block was essential, and saw this as the basic mechanism of fibrillation. The general idea that fibrillation might be explaned as a simple circus movement, was strongly contested by Lewis "at first.

It was not until he attempted to follow the path taken by the excitation wave in atrial flutter, that he acknowledged the significance of experiments on ring preparations. He mapped atrial flutter in dogs with a single roving probe and after visualizing a probable circuit around the orifices of the caval veins, he accepted the idea that the excitation wave followed a circular path, reentering its own track and thus perpetuating the arrhythmia. ${ }^{34}$ 

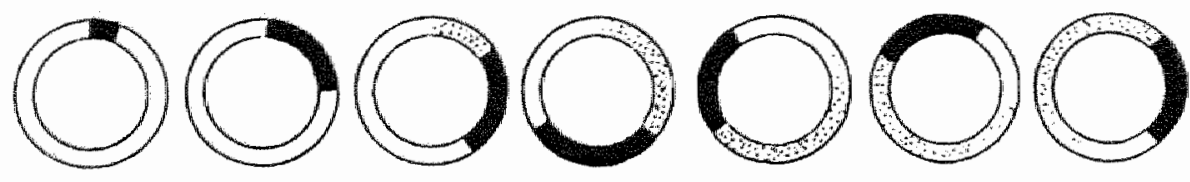

Nigure 1.3

A diagram to illustrate the establishment of circus movement in a ring of muscle. The ring is stimulated at the top. Conduction is blocked towards the left due to the high rate of stimulation, but cain continue to the right in a clockwise fashion (black area). Where the wavefront has past, it leaves 4 tall of relative refractory tissue (dotted area). Propagation of the wavefront has been sufficiently slow to allow the tissue at the top to recover from refractoriness: (white area). Therefore, the wawe of excitation may spread a second time ower the same tract of tissue, and may remain to do so unless it is interfered with. From Mines ${ }^{23}$ Figure $24 b$.

\section{Mother Wave - Daughter Waves}

The study of atrial fibrillation ${ }^{25}$ was a natural follow-up to the analysis of flutter. Jewis regarded atrial fibrillation as an advanced state of impure flutter. He knew that the central "mother wave", following a constant anatomical path, caused the regular atrial complexes of pure atrial flutter. The rate of impure atrial flutter was shown to be faster, causing irregular atrial complexes. Lewis noted that these irregularities were probably caused by disturbances of conduction of centrifugal "daughter waves", and concluded that the oscillations of fibrillation had a similar origin." He showed that at high rates the waves propagated through areas containing refractory fibers; when a wave approached tissue in its period of partial refractoriness, only a percentage of fibers responded, tesulting in an irregular recovery of responsiveness. In such cases the centrifugal daughter waves were forced to travel a sinuous course through the atria. ${ }^{35}$ Nonetheless, Lewis still contested the notion that a simple circus mowement around an anatowical obstacle sufficed to describe atrial fibrillation. Instead he now adopted the view that in fibrillation the sinuosity of course was generalized and affected not only the daughter waves but also the central mother wave. The obserwed differences between coarse and fine atrial fibrillation, ${ }^{5,}, x_{n}$ when would be explained by an increase in the amount of simuosity. ${ }^{2, n}$

As was discussed previously, Lewis initially hypothesized that fibrillation consisted of numerous ectopic impulses in the atrial walls? The probability of the simultaneous presence of multiple changing circuits within one mass of muscle 
was mot commented upon by Garrey" and Mines" but remained a possibility in their theories. When Lewis accepted the concept of circus movement ine questioned whether an increased variation in the single mother wave would be sufficient to acconnt for the phenomena of fibrillation. ${ }^{2}$ He considered severat types of circus movements: from multiple circuits, completely or transienty independent of each other, to a single fully erratic circuit, sometimes transiently breaking into different circuits. In his view, the available dala at that time indicated that the excitation waves during fibrillation spread as coordinate waves. though they traveled along paths that were often wery sinuous. This wiew was supported by his observation that electrograms from simultancous epicandial leads showed deflections that appeared simultaneously. He only saw one exception: from time to time a single excitation wave appeared to produce not one but two deflections (Figure 1.4). He noted that the electrognams in atrial fibrillation became very complex from time to time, suggesting that the twin deflections originated from more than one source. However, he found it difficult to imagine that such double potentials represented two excitation waves activating the same muscle fibers: the interwal between the two was often too short for the refactory period. He suggested that both deflections were produced by two excitation waves from opposite directions that conducted through the same sheet of tissue, but not precisely through the same strands of fibers. Fle explaned this phenomenon by partial or complete hindrance (block) of excitation waves traveling in partially refractory muscle. He assumed that a single excitation wave striking refractory tissue would split on such a bartier and that the two crests would meet again later on. Another possible explanation was that the wave would turn around the barrier until it became responsive. This is actually the first suggestion of a possible reentrant circuit around a functional obstade. ${ }^{25}$

Lewis then compared fibrillation electograms to those of impure flutter, and rejected the idea of multiple circuits. He conduded that the complexilies of the curres were compatible with a single source of origin and were to be explained on the ground of sinuous circus movement. In Lew is 's eyes, the most convincing argument against simultaneous circus movements, was the appearance of wellformed oscillations in the Eco during fibrillation, waves which in shape, incidence, and rate were very much like flutter waves. This finding left him no doubt that they were similarly derived. ${ }^{25}$ Another argument he brought forward was that the frequency of atrial fibrillation is approximately $50 \%$ faster than fluter (450 ws 300 beats/min). He believed that multiple circuits would yield movements 

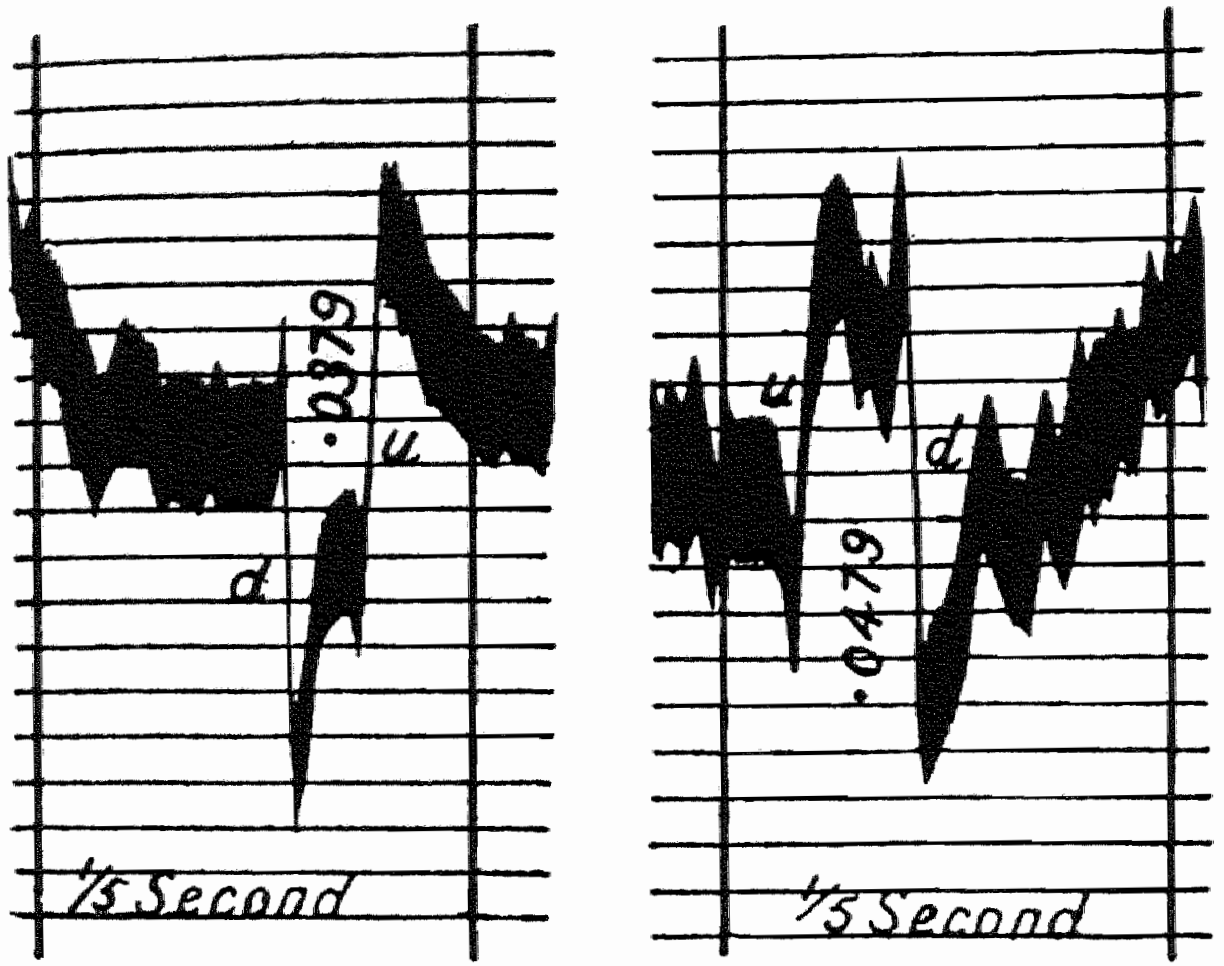

Figure 1.4

Double or "twin" deflections in two electrograms from a fibrillating dog heart. From Lew is Figure 4.

at much higher rates, whereas on the other hand he lelt that the variations in frequency $(10 \%$, coud be adequately explained by variations of a single circuit alone. This last argument seems illogical when one takes into account the rate related slowing of conduction. Lewis had already shown that conduction velocity

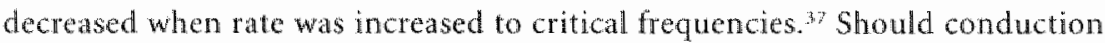
velocity have remained stable, then a circuit with hall the diameter of a fluter cincut would have a revolution time of half the duration. This would indeed result in a rate much faster than atrial fibrillation (600 beats/min). However, at that rate a decrease in conduction velocity of $25 \%$ is feasible. ${ }^{37}$ This would increase the revolution time by $25 \%$, resulting in a rate of 450 beats/min. Since a circuit of hath 
the diameter occupies only a quarter of the space, four of these circuits might coexist at a normal fibrillation rate. Lewis did not take into account the rate dependent decrease in conduction velocity, and therefore mistakenly discarded the possibility of multiple circuits. However, this does not exclude he possibility of a single sinuous circuit as the perpetuating mechanism of atrial fibrillation.

It is to be undersitood that the work so far described, left the ultimate nuchanism of fibrillation still in the stage of hypothesis. Actual circuits were never completely visualized. In an attempt to do so, Lewis used the indirect method of vector analysis to demonstrate circus movement in two patients with atrial fibrillation and concluded that a single shifting circult existed around the two atrial cavities.

Thus, Lewis's understanding of atrial fibrillation was, that it is not necessarily dependent upon structural abnormalities of the atria. As the perpetuating mechanism he considered both theories of multiple ectopic foci and multiple circuits, but rejected them after careful reasoning and precise experimental work. He finally came to the conclusion that atrial fibrillation was caused by angle mother wave circulating through a more or less irregular track. He was inconclusive about the question whether these circuits were functionally or anatomically determined. "The striking irregularity of atrial fibrillation was explained by the high rate causing the mother wave and her offsprings to travel through partially refractory tissue, thus creating a sinuous course.

Despite Lewis's arguments in favor of the theory of circus movement as the cause for atrial fibrillation, many still believed in the concept of ectopic foci, which originally was also supported by Lewis. Only in 1947 was the actual presence of both ectopic foci ${ }^{39}$ and circus movement around an anatomic obstacle accurately demonstrated experimentally in vivo hearts. Broth theories differentiated between flutter and fibrillation in terms of the frequency of the governing agent. However, becase of the irregular atrial activity in fibrillation it was difficult to prove any of the several mechanisms. It was not until 1959 that more conclusive data on this matter were published by Moe.4:

\section{GondON R MOR}

Moe was of opinion that both ectopic foci and circus movement could have their role in clinical atrial arrhythmias. Since both had been produced experimentally, "nature might sometimes perform similar experiments". He found it conceivable that a circus movement could exist in a path in which the refractory period was 
shorter than in the rest of the atrum. In this situation the circuit length might be short enough to result in al frequency of impulse discharge exceding the capacity of the atria to follow regularly. It was equally possible to him that an ectopic pacemaker could fire at a frequency taxing the properties of the surrounding atrial muscle. However, Moe found it difficult to believe that either of these medhanisms would be sufficiently stable to persist for years as fibrillation often does."

\section{Atrial Fibrillation is Independent of its Initiating Agent}

Moe rasoned that although fibrillation might be started by ectopic impulses or even by an early sinus beat, fibrillation might persist after the initiating mechanism had stopped. It had been shown that the initiating agent could be a stimulus from electrodes, ${ }^{40}$ or a rapidly discharging aconitine focus ${ }^{39}$ Spontaneous occurrence of atrial fibrilation was thought to be initiated by a premature beat or an impulse circulating around an obstacle. All of these methods of inducing fibrillation had one feature in common: the atrium was reexcited early in its relative refractory period. ${ }^{\prime}$ Moe and Abildskov demonstrated that under certain conditions fibrillation, once induced, indeed could continue after removal of its initiating agent. An open chest dogs, atrial fibrillation was induced during vagal stimulation with electrical stimuli delivered to the tip of the right atrial appendage. It was shown that fibrilation persisted after the preciptating focus (the external stimulator) was turned off. "The possibility was considered that rapid atrial stimulation created an ectopic focus that continued to fire after the stimulator had been stopped. In such a case, isolation of the atrial appendage by clamping its base must result in immediate cessation of atrial fibrillation in the body of the atria, whereas rapid activity in the appendage should continue. However, the experimental results showed the opposite; applying the clamp had no eflect on the body of the atria, it continued to fibrillate, whereas in the appendage the rate slowed or the beating stopped to beat altogether. This effect was independent of the site of stimulation."

In another experinent atrial fibrillation was induced by injection of small wolumes of aconitine solution into the atrial muscle near the tip of the right atrial appendage. When in this case the clamp was applied to the base of the atrial appendage, the body of the atria immediaty resumed sinus rhythm, whereas in the clamped off appendage fibrillation was usually replaced by a regular tachycardia. The experiment was extended with vagal stimulation, thereby shortening the atrial refractory period. Under these conditions, application of the 
clamp did not result in cessation of fibrillation in the body of the atrium. Instead, fibrillation induced by either agent was self-perpetuating in the body of the atrium. Moe, therefore considered the arrhythmia a stable state conpatible with the normal parameters of the atria but independent of its origin regardless whether this was a circus path or an ectopic focus. An explanation was needed, not in terms of the initiating agent, but in terms of properties of the atria to permit independent and self-sustaining fibrillation. ${ }^{41}$

\section{The Multiple Wavelet Hypothesis}

Earlier it had been shown that, although depolarization in fully excitable tissue propagated uniformly in all directions from its site of origin, repolarization did mot progress so evenly because the refractory period does not have precisely the same duration in all atrial fibers. ${ }^{24}, 32,+10$ Moe reasoned that, if the spread of recovery is inhomogeneous, some fibers would already be excitable while nearby fibers would still be refractory. ${ }^{1 k}$, 41 Therefore a second premature response would propagate irregularly. Those fibers that where in an advanced state of recovery would conduct rapidly, whereas those fibers that were still partially or totally refractory would be delayed or altogether blocked. Further repetition of impulses would only increase the spatial and temporal dispersion of refractoriness, and an orderly spread of excitation would no longer be possible. In such conditions a "grossly irregular wavefront becomes fractionated as it divides about islets of refractory tissue, and each of the daughter wavelets might now be considered as independent offspring. Such a wayelet may accelerate or decelerate as it encounters tissue in a more or less advanced state of recovery. It may become extinguished as it encounters refractory tissue; it may divide again or combine with a neighbor; it may be expected to fluctuate in size and change in direction. Its course, though determined by the excitability or refractoriness of surrounding tissue, would appear to be as random as a Brownian motion. Fully developed fibrillation would then be a state in which many such randonly wandering wavelets coexist." ".1

Perpetuation of atrial fibrillation would then depend upon the number of wavelets simultaneously present in the atria. A small number of wavelets would have a high chance of fusing or dying out, resulting in restoration of sinus rhythm. On the other hand, a large number of wavelets would have a high chance of perpetuating the arrhythmia, since it seems unlikely that all elements would die out simultaneously. According to this multiple wavelet hypothesis, perpetuation of 
atrial fibrillation is dependent upon factors that influence the average number of wavelets suchas atrial tissue mass, refractory period, and conduction velocity. A large atrial surface can contain a larger number of wavelets, whereas a shor refractory period or a low conduction velocity would also allow more wavelets to cocxist in the atria. On the other hand, a long refractory period would soon leave the atria totally refractory, thereby interupting fibrillation. Also, if exery impulse would conduct rapidly to the borders of the atria, the disorganization of atrial activity would not occur. ${ }^{3.4}$ "Mo did not comment upon the possibility that a wavelet might be blocked ewen when conduction is fast. This happens when the tissue is still refractory from activation by another wavelet. However, also in such a situation conduction velocity is an important factor since a slowly conducting wavelet gives the tissue ahead enough time to regain excitability. Therefore, when conduction velocity is low, a wavelet can circulate in a smaller mass of tissue.

Moe stated that multiple wavelets could already exist in normal atria, since a certain degree of natural inhomogeneity exists. ${ }^{29}, 32$, whe refractory period was found to be a function of cycle length, and as a result premature excitation led to irregular wavefronts. Expermentally it had been shown that cutting cardiac tissue into picces smaller than 1 gram promptly stopped ventricular fibrillation ${ }^{32}$ and intraatrial reentry. Comparison between large adult cattle and horses, young calves, and adult goats and sheep, showed that sustained atrial fibrillation was only inducable in the langer animals. Atrial fibrillation seemed to perpetuate as a function of atrial tissue mass rather than age related structural changes in the atria. Adult human atria are somewhat smaller than the atria of adult catte but larger than those of sheep and goat. This may explain why atrial fibrillation usually terminates spontaneously within minutes when it is produced in a normal human heart. However, atrial fibrillation becomes persistent when the atrial refractory period is shortened by vagal stimulation or administration of acetylcholine. Aabbit atria, which under normal conditions are too small to fibrilate, will exhbit sustained fbrillation when the refractory period is reduced by drugs" or dilatation. ${ }^{4 * 4}$

\section{A Computer Model of Atrial Fibrillation}

A direct test of the multiple wavelet bypothesis was difficult, and at that time experimentally impossible. Circumstantial evidence was sough by means of a computer model." sid a mathematical model, a two-dimensional piece of 
cardiac tissue with nomal electrical properties was simulated. Discrete units were arranged in a regular hexagonal array, thus providing each unit with six neighbors (Figure 1.5). A unit conducted evenly to each of its neighbors when excited. If excitable, the neighbors fired in turn after a delay determined by their state of excitability. If refractory, they were not influenced. The model tissue was given a nonhomogeneous distribution of refractory periods that was dependent upon the preceding cycle length. This was calculated as: $R=K \sqrt{C}$. R representing the duration of refractoriness in an individual "unit", $K$ being a random range of values, and $C$ the duration of the preceding cycle length. During the period of relative refractoriness, the conduction velocity was a function of the level of excitability. The values that Moe assigned to these parameters, were based upon estimates made in dog atria. An attempt was made to assign a "fibrillation number" that, like the Reynolds number; would define the critical circumstances required for atrial fibrillation. Such a "fibrillation number" F was calculated as follows:

$$
F=\frac{L \delta_{\mathrm{r}}}{K^{2}}
$$

$L$ is the linear dimension characteristic of size and shape of the matrix, $\delta$, represents the standard deviation of local conduction velocities, and $\bar{K}$ is the average value of $K$ 's in the refractory period. "The results of the model mimicked the features of fibrillation in the living system in many respects. Self-sustained activity could be induced by a brief period of repetitive stimulation, and it maintained itself after the external stimulus was withdrawn. The electrical activity was highly irregular. Periodic repetition of excitation patterns did not occur; instead multiple $(23-40)$ small wavefronts meentered already previously activated cells at varying sites (Figure 1.5). The reentrant circuits constantly died out and were replaced by others, resulting in a continuous shift in position, frequency, width, direction, number, and velocity of the circuits. In the computer model, ir regular activity was only possible if the refactory period was non uniformly distributed, whereas, like in true fibrillation, prolongation of the average refractory period ( $K$-values) led to a coarsening and eventual termination of $A$. When conduction velocity was made homogeneous, the arthythmia became more regular. Reduction of the tissue mass could stop the arthythmia, but when the original area was large enough, fibrillation continued in both halves after bisection of the tissue. When an obstacle of sufficient size was made in the tisstue sheet, atrial frbrillation was replaced by a regular circus movement. 40.52 

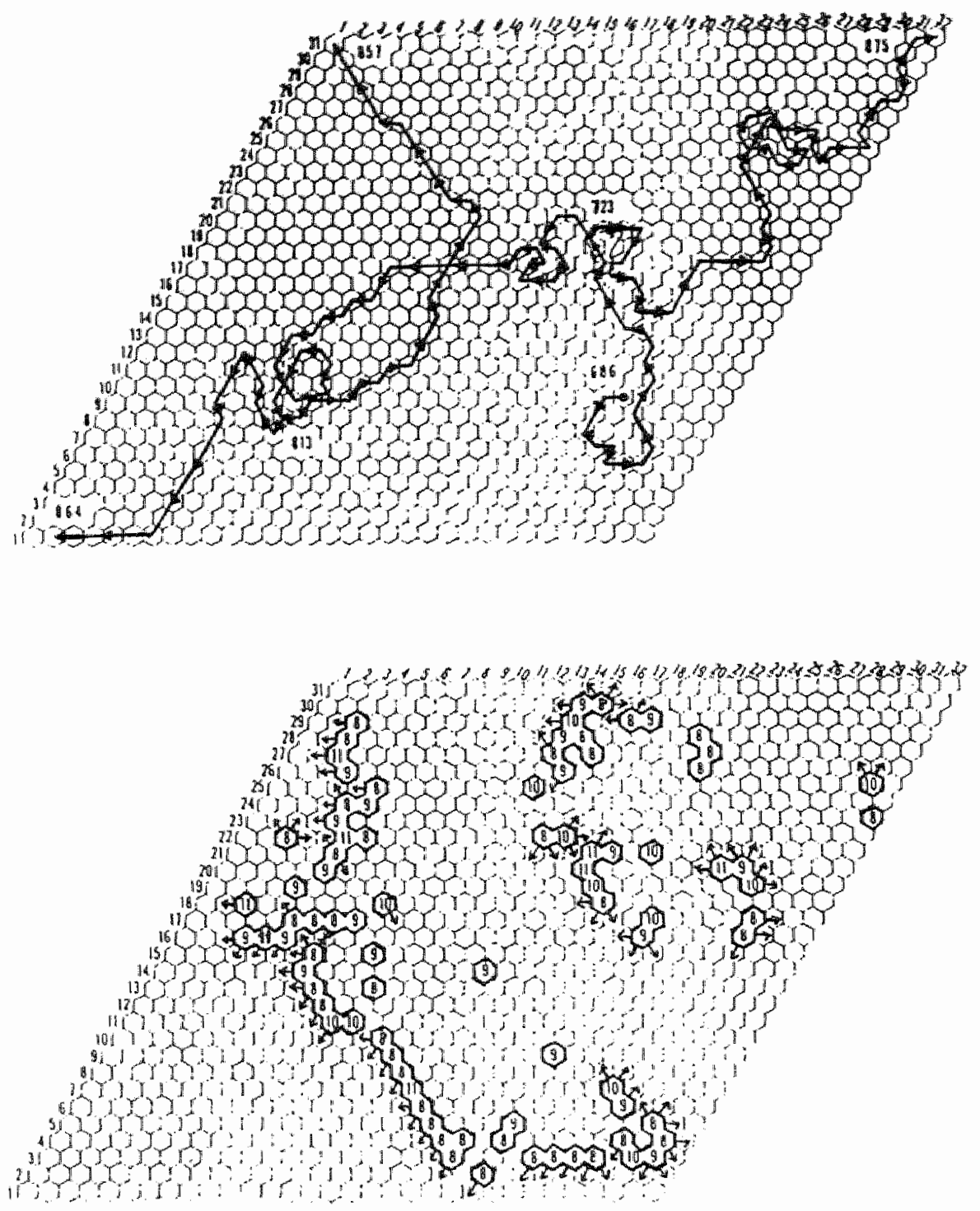

Figure 1.5 A computer model of atrial fibrillation. Upper panel: Pathways of three wavelets that arise from excitation of one unit at time 686 Arrows show the direction of condwction. Lower panel: wavefronts mapped at time 528 . The numbers indicate firing time $(8=528,9=529$, etc) At this moment, 30 wavefronts are simultaneously present. 20 of which are propagated further (indicated by small arrows), and 10 die out (no arrows). The mathematical tissue has an irhomogeneous distribution of refractory periods, that is dependent upon the cycle length of the preceding activation. This results in variable functional blocks, causing an erratic course of wavelets and fractionation of wavefronts into independent offsprings. From Moe, as Figures 6 and 7. 
The computer model of course could not determine the mechanism of the naturally occurring disorder, nor did it exclude possibilites other than the multiple wavel hypothesis. It did, however, show that something akin to atrial fibrillation could sustan itself in a limited system; featuring inhomogeneities, conparable to those observed in normal dog atria. Alterations expected to reduce the persistence of Af (increased refractory period, decreased tissue size) reduced the number of wavelets. ${ }^{49-5}$ The model also illustrated that reentrant circuits could be generated without the involvement of an anatomical obstacle. It was assumed that islands of refractory tissue could provide the substrate for citcuit formation, but it was thought that such islands resulted from pathological alteration of the properties of the tissue. In such cases the circuits would be located at certain anatomically preferential sites. The model showed that completely functionally determined circuits could exist, that continuously shifted in position.4

Moe regarded atrial fibrillation as an arrhythmia that was independent from its origin. As long as the local refractory periods were sufficiently short and heterogeneous, a premature impullse could generate multiple wavelets. During stable atrial fibrillation many simultaneous wavelets would propagate in constantly changing directions, their path being determined by functional obstacles. "Arrest of fibrillation may be considered as a probability phenomenon. If the total number of wavelets which course randomly throughout the atrial muscle is large, the likelihood that they may fuse with each other to render the whole of the atria excitable or inexcitable is small; if, on the other hand, the number is small, the chance of fusion becomes correspondingly large. Any lactor therefore, which increases the average number of wavelets will tend to perpetuate the arthythmia, while any agent which reduces the number will favor chance recovery. The parameters which may be assumed to be important are total mass of the atria; refractory period; conduction velocity; and excitability." $A t$ this time, still many aspects remained unclear; the critical number of wavelets necessary to perpetuate fibrilation and, mote importantly whether multiple independent waveletts existed at al].

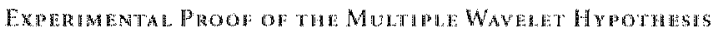

To prove the multiple wavelet hypothesis direct testing of the theory whas required. However, this had to wait unt progress in electronics and computer technology enabled sufficiend simultaneous recordings of the electrical activity in order to 
reconstruct the actiwation patterns during atrial fibrillation. Such a mapping rechmique was first used by Allessie et al. ${ }^{53}$. acetylcholline-induced atrial flutter and atrial fibrillation in isolated Langendorf perfused canine hearts. 37 The results of these studies were later confirmed and extended by others. 5 tion

An example of the activation maps acquired during atrial fobrillation is given in Figure 1.6. Seven consecutive maps from both the right (RA) and the left atria (LA) are shown, depicting the electrical activity on the endocardial surface during atrial fibrillation in the dog. The propagation of impulses is wisualized by isochrones of 10 miliseconds in a gray scale code. The general direction of the wavelets is indicated by white arrows. Asterisks indicate sites of new impulses originating from the other atrium. For comparison, at the lower right corner of each panel, the activation sequence during normal sinus rhythm is given. Each map shows multiple small wavelets that are constantly changing in width, ranging from 0.5 to $4 \mathrm{~cm}$. These wavelets propagate randomly through each atrium, and from one atrium to the other. They constantly change direction, turning around functional arcs of conduction block, or they divide, collide, or fuse. Frequently wavelets die out by collision or fusion with another wavelet or because they meet refractory tissue or the anatomical borders of the atria. The lifetime of the individual wavelets is rather short, not more than few hundred milliseconds on the average. At time 300 milliseconds the two wavelets in the right atrium are extinguished simultaneously (RA, map $1:$ ). However, atrial fibrillation continues in the left atrium, after a short time a new wavelet enters the right atrium and atrial fibrillation restarts ( $\mathrm{kA}$, map $\mathrm{F}$ ). New wavelets are also formed by division of an existing wavelet at a line of conduction block. Other studies showed that new wavelets can also arise from the sinus node area ${ }^{62-6}$ or from ectopic foci. Complete circuits are rarely visible ( $\mathrm{R}$, map $\mathrm{C}$ ), and an impulse rarely follows the same circular route more than once. In most cases wavelets re-excite an area that was activated before by another propagating wavelet. This type of reentry was described by Hofman and Rosen"s as "random reentry". On the average, six wavel ats simultancously present; three in the left and three wavelets in the right atrium. It was estimated that the critical number of wavelets for perpetuation of atrial fibrillation is between three and six.s.5.

'This estimate is in contrast to the 23 to 40 wavelets shown by Moe in his computer model of arrial fibrillation (Figure 1.5) and it was concluded that the computer model used a tissue mass that was a number of times greater than the climensions 
of canine atria. This is in concondance wrth Moes finding that division of the computer tissue in half does not intermpt the arrhythmia.

From these experimental mapping studies it was concluded that Moe's multiple wavelet hypothesis was inded an important mechanism of atrial fibrillation. ${ }^{\text {s }}$ However, these findings do not exclude that other mechanisns are involved as well. In another mapping study of atrial fibrillation in dogs and w Pw patients in some cases the fibrillatory wavelets appeared to be daughter waves from a single reentrant mother wave.

Detailed investigation of activation patterns during fibrillation, so far, has been limited to experimentally induced atrial fibrillation in animal hearts. ${ }^{5-4}$ in humans, only a few cases have been studied. 5 . Also, still little is known about activation patterns during chromic atrial fibrillation. The main objective of the present thesis is to systematically analyze electrical activation during induced atrial fibrillation in humans and to compare the atrial excitation during acute and chronic atrial fibrillation in goats. 
A

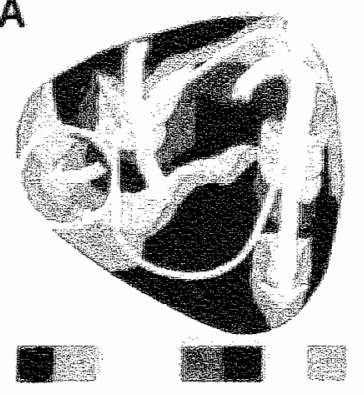

0

80

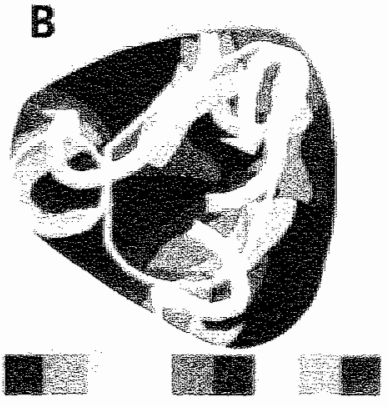

80

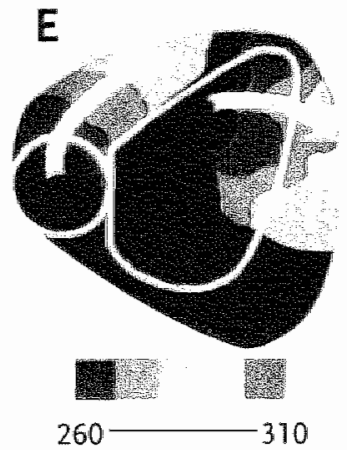

D
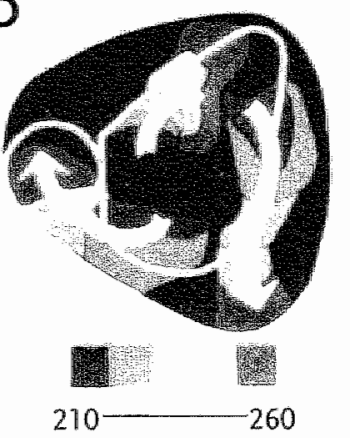

Q

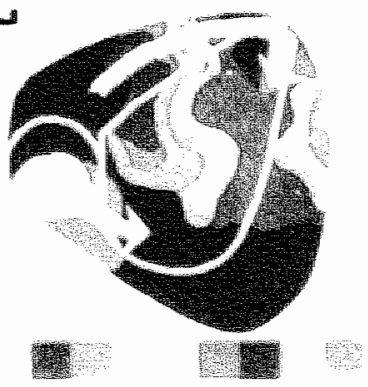

410 490
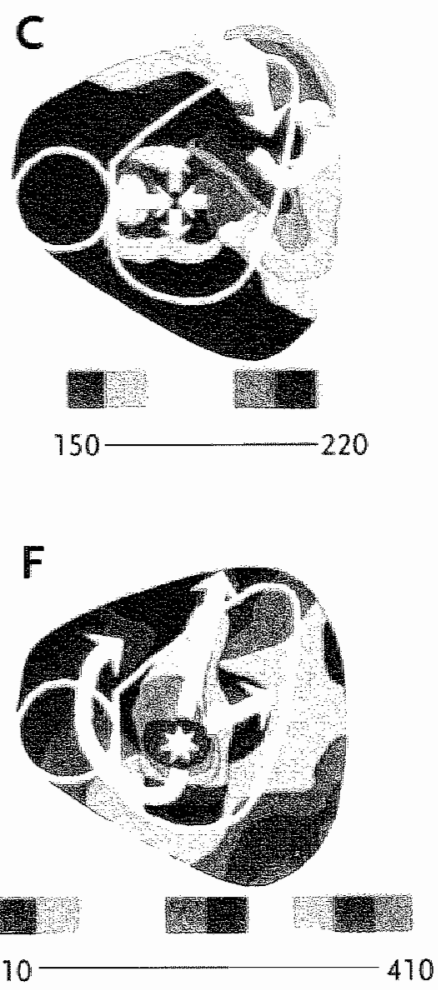

SINUS BEAT

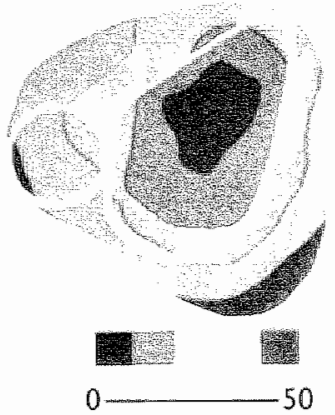

Figure 1.6 A series of consecutive activation maps covering the spread of excitation in the right and left atria during half a second of atrial fibrillation in the dog. Numbers at the bars below the maps are in milliseconds and indicate the time of activation of the isochrones. See text for further description. From Allesie,"s Figures 7 and 8 
A

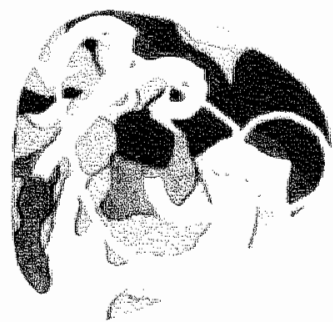

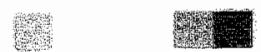

0

D

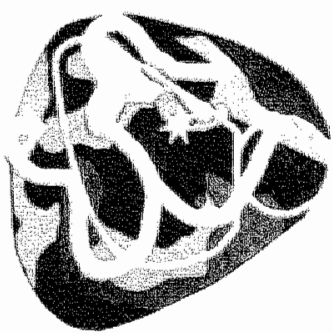

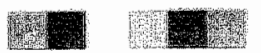

B
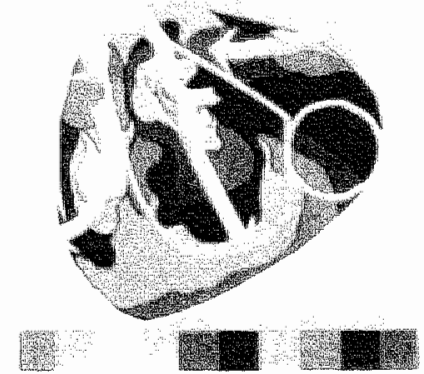

170

E

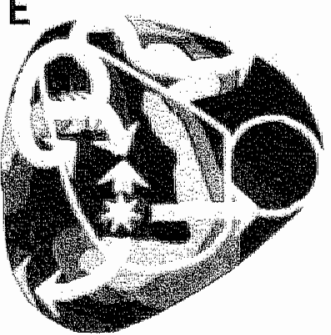

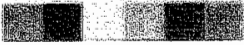

C
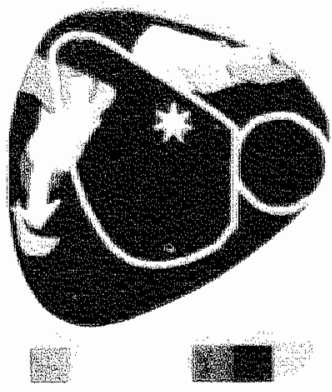

140

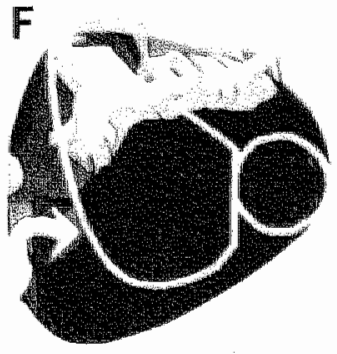

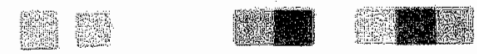
$410 \quad 380$

SINUS BEAT

G
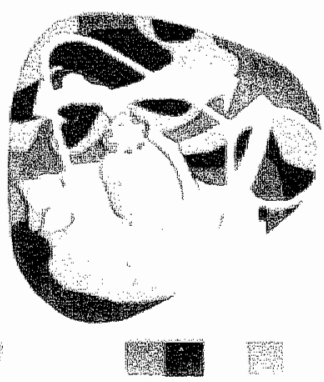

410 490
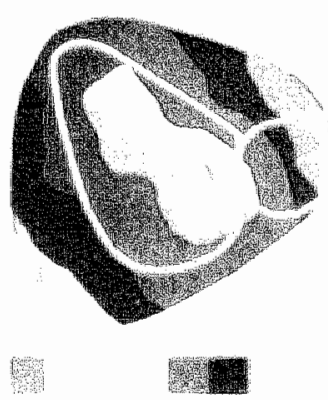

0 $-50$ 



\section{Chapter 2 | High-Density Mapping of}

Electrically Induced Atrial Fibrillation IN HUMANS |

1.

Karen T.S. Konings, MD*; Charles J.H.J. Kirchhof, MD, PhD; Joep L.R.M. Smeets, MD, PhD*; Hein J.J. Wellens, MD, PhD; Olaf C. Penn, MD, PhD; ;nd Maurits A. Allessie, MD, PhD

From the deparments of Phisiologyt, Cardiology and Cordiopulmonary Sugery "Candiowasculan Reseatch Institute Matastricht 
The mechanisms underlying atrial fbrillation in humans are not yet fully understood. In the beginning of this century, it was believed that the irregular contractions of "the atria were calsed by either single or multiple ectopic foci.67.69 "This concept was first challenged in 1920 by Lewis" who stated that during atrial fibrillation, the incoordination of the contracting fibers may be held to result from the impact of contraction waves and the production of localized areas of block." Also, Garrey concluded that reentry had to be the mechanism of atrial fibrillation. The debate about ectopic foci versus reentry continued, and on the basis of computer simulations, Moe" hypothesized in 1962 that a "grossly irregular wave front becomes fractionated as it divides about islers or strands of refractory tissue, and each of the daughter wavelets may now be considered as independent offspring. Fully developed fibrillation would then be a state in which many such randomly wandering wavelets coexist." Experimental evidence for this multiple wavelet hypothesis was provided by a number of studies in which the excitation of the atria during atrial fibrillation was actually mapped ${ }^{-6}$. these studies showed multiple wavelets wandering around natural anatomical obstacles and functional arcs of conduction block. In some cases, the wavelets appeared to be offsprings of a single reentrant circuit. ${ }^{36}$ However, until now mapping of atrial. fibrillation in humans has been limited to a small number of patients, and no systematic analysis of mapping data has been done.

With the use of the surface ECG, atrial fibrillation has been divided clinically in "coarse" and "fine" fibrillation." atrial fibrillation based on the characteristics of the bipolar atrial electrogram.

The objective of the present study was to systematically analyze the patterns of activation using high resolution mapping during electrically induced atrial fibrillation in a relatively large group of young pattents being operated on for transsection of an accessory atrioventricular pathway.

\section{Mernoos}

\section{Patient Population}

The study included 25 patients with Wolf-Parkinson white (w w w) syndrome who were undergoing surgery for symptomatic or drug refractory tachycardia at the Academic Hospital of Mastricht between March 1988 and June 1990 . Their 
average age was $32 \pm n$ years (range, 12 to 57 years). Sixteen patients were male and nine were femali. In none of the patients were cardiac abnomalities other than the wPw-syndrome found by evaluation of the chest radiograph and cotonary catheterization. The left atrial size as detemined by echocandiography was notmal in most patients. In three patients $(22,23$ and 25$)$ the left atrium was moderitely enlarged.

In all patients, an invasive electrophysiologic study was performed before surgery. Durng this study, circus movement tachycardia was induced (except in patierts 5 and 19) and the location of the accessory pathway(s) was determined. A left lateral accessory pathway was present in 15 patients, a left posteroseptal pathway in four, a right lateral pathway in wo, a posteroseptal pathway in two, and a right posteroseptal pathway in one. In one patient, two accessory pathways existed; one located in the right free wall and one located left laterally. The atrial refractory period during pacing at 500-millisecond intervals was nomal. In seven patients, paroxysms of atrial fibrillation were documented by $\mathrm{ECG}$ or Holter recordings. These 7 patients did not have a significantly different atrial refractory period or left atrial sizes $(p>.462)$. In Table 2.1 , clinical chatacteristics of the patients are given. Antiarthythmic medication was discontinued four to five days before surgery. None of the patients were taking amiodarone. Informed consent for induction of atrial fibrillation and mapping was obtained before surgery.

\section{Experimental Protocol}

The patients were anaesthetized with fentanyl (50 to $100 \mu \mathrm{g} \cdot \mathrm{kg}^{-1}$ ) and allentanil (2 $\left.\mu g \cdot \mathrm{kg}^{-1} \cdot \mathrm{min}^{-1}\right)$ and propofol $\left(2 \mathrm{mg} \cdot \mathrm{kg}^{\cdot 3} \cdot \mathrm{h}^{-1}\right)$. The chest was opened by median sternotomy and a pericardial cradle was made. Four bipolar electrodes were attached to the right and left atria and to each of the ventricles for bipolar recording and stimulation. Epicardial mapping of the free wall of the right atrum was performed during sinus rhythm, rapid atrial pacing, and induced atrial fibrillation, before the patients were put on cardiopumonary bypass and before cryo-ablation of the accessory pathway(s). A spoon shaped clectrode (diameter. $3.6 \mathrm{~cm}$ ) was used that consisted of 244 unipolar electrodes (silver wires; diameter, $0.3 \mathrm{~mm}$ ) arranged in a regular octagonal array (interelectroda distance, $2.25 \mathrm{~mm}$ ) (Figwre 2.1). A silver plate (diameter, $2.5 \mathrm{~cm}$ ) positioned in the thoracic cavily was used as an indifferent electrode. The mapping electrode was positioned in the middle of the right atrial free wall and was kept in place manually by the surgeon applying light constant pressure to the atrium. In this way, usually more than $90 \%$ 
Table 2.1 Characteristics of Patients with Electrically Induced Atrial Fibrillation

\begin{tabular}{|c|c|c|c|c|c|c|c|}
\hline & Age & ser & $\begin{array}{l}\text { Lonation of } \\
\text { Pathway }\end{array}$ & AESRP & LAs size & $\begin{array}{c}\text { Documented } \\
\text { Af }\end{array}$ & $\begin{array}{l}\text { Duration } \\
\text { of Analyred } \\
\text { Episode of Afy }\end{array}$ \\
\hline Patrow wo. & $(v)$ & & & $(\mathrm{ms})$ & (mira) & 7 & (s) \\
\hline 1 & 18 & $\mathbb{p}$ & $R L+L L$ & $\mathrm{NA}$ & 39 & - & $>120$ \\
\hline 2 & 27 & $M$ & LL & 230 & $\mathrm{NA}$ & - & 420 \\
\hline 3 & 23 & $M$ & $R L$ & 260 & NA & - & 40 \\
\hline 4 & 16 & $M$ & RL & $\mathrm{NA}$ & NA & - & 60 \\
\hline 5 & 28 & $t^{*}$ & $\llcorner\mathrm{PP}$ & 190 & 28 & - & $>120$ \\
\hline 6 & 27 & $M$ & $L$ & 220 & 43 & - & $>120$ \\
\hline 7 & 45 & M & $\mathscr{L L}$ & 230 & 40 & 4 & 540 \\
\hline 8 & 20 & $M$ & LP & 260 & 38 & - & 120 \\
\hline 9 & 57 & $M$ & $L L$ & 200 & 42 & $\stackrel{4}{2}$ & 30 \\
\hline 10 & 18 & $M$ & $L L$ & 210 & 40 & - & 60 \\
\hline II & 36 & F & $L L$ & NHA & 32 & - & 35 \\
\hline 12 & 43 & $M$ & LL. & 220 & 36 & + & $>120$ \\
\hline 13 & 45 & $\mathrm{M}$ & LL & 200 & 36 & + & $>300$ \\
\hline 4 & 25 & $M$ & $L L$ & 220 & $\mathrm{NA}$ & + & $>60$ \\
\hline 15 & 27 & $M$ & LL & $\mathrm{NA}$ & 43 & - & $>1.80$ \\
\hline 16 & 37 & $M$ & LL & 250 & $\mathrm{NA}$ & - & 60 \\
\hline 17 & 29 & $M$ & L.P & 180 & 30 & - & 180 \\
\hline 18 & 34 & $M$ & $\mathrm{LL}$ & 160 & 44 & - & $>180$ \\
\hline 19 & 12 & $\mathrm{~F}$ & ps & 260 & 37 & + & $>600$ \\
\hline 20 & 31 & $F$ & $\mathrm{RE}^{\mathrm{P}}$ & 200 & $\mathrm{NA}$ & - & $>60$ \\
\hline 21 & $5 \pi$ & $\mathrm{F}$ & LLL. & 240 & 32 & 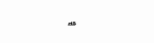 & 300 \\
\hline 22 & 3.8 & F & PS & 200 & 45 & - & $>260$ \\
\hline 23 & 3.4 & $M$ & LiL & 260 & 50 & + & $>110$ \\
\hline 2. 새 & 44 & F & LP & NA & $\mathrm{NA}$ & - & 180 \\
\hline 25 & 33 & f & $\mathrm{LL}$ & 220 & 46 & - & 60 \\
\hline Avgres & $32 \pm 11$ & $16 m+9 z^{2}$ & & $221 \pm 29$ & $39 \pm 6$ & $7+$ & $>173 \pm 154$ \\
\hline
\end{tabular}

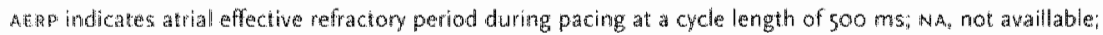

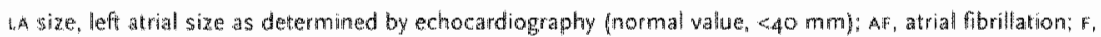

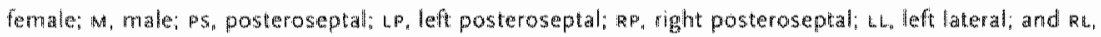
right lateral. 
of the electrodes recorded adequate electrograms of sufficient amplitude without motion artifacts. At the edge of the mapping electrode, some electrodes sometimes yielded low-amplitude signals, either due to fat at the atrioventricular sulcus or because the edge of the electrode did not make good contact with the atrium. Because this only occurred at the edge of the electrode, the only consequence was that the map became slightly smaller than the actual size of the electrode array. We also tried to record from the free wall of the left atrium. However, because of the poor exposure of the left atrum, the left atrial recordings were of sufficient quality in only eight of 25 patients. All electrograms were individually amplified (gain, 150 to 1000 ), filtered (bandwidth, $210500 \mathrm{~Hz}$ ), multiplexed (sampling rate, $1 \mathrm{kHz}$ and a converted ( 8 bits). Bipolar reference electrograms from the right and left atria and the right and left ventricles and aco leads I, II, II and avr, were recorded simultaneously. All recordings were stored on videotape (Sony sL-CoEs) for subsequent analysis.

Atrial fibrillation was induced either by incremental atrial pacing or by introduction of one to thee early atrial premature beats. Constant current pulses of 2 millisecond duration and twice-diastolic threshold were used. The duration of electrically induced atrial fibrillation varied from less than three seconds to more than ten minutes. In all patients, atrial fibrillation terminated spontaneously, except in one patient, in whom it was terminated by cardioversion. Only episodes of atrial fibrillation lasting longer than 30 seconds were used for analysis. In most patients ( $n=20$ ), only one episode of "sustained" atrial fibrillttion was induced. In case of multiple induction of atrial fibrillation, the longest episode was selected for analysis. The average duration of the analyzed episodes was more than three minutes.

\section{Activation Maps and Interval Histograms}

During off-line analysis time windows of 22 seconds of atrial fibrillation were selected from tape, and the signals were transferred to a personal computer (Olivett 386 ) for detaled analysis. In three patients, only four seconds were analyzed because of short duration of atrial fibrillation. Because it is feasible that both during initiation and before termination of fbrillation, transients may occur between more or less complex patterns of fibrillation, the first and last 12 seconds of atrial fibrillation were excluded from analysis. During the 12 second sample of fibrillation selected for analysis, the pattern of atrial fibrillation appeared to be stable as judged from the cycle length and degree of irregularity of the different 
dectrograms. The software used for analysis of the 244 recorded unipolar electrograms included an algorithm for automatic detection of the intrinsic negative deflections of the electrograms, ${ }^{73}$ generation of color-coded activation maps, and interactive editing of local activation times. Isochrones of 10millisecond intervals were drawn by hand. Intra-atrial conduction block was defined as an apparent local conduction velocity less than $7.5 \mathrm{~cm} / \mathrm{s}$ associated with a change in direction of propagation distal to the line of block. ${ }^{74}$ A detailed description of the mapping system has been given.75.76 Fibrillation interval histograms of the right atrium were made using five electrograms of the mapping area; one recorded at the center and the others at the middle of each quadrant of the mapping electrode. The wariation in local fibrillation interval was expressed as the difference between the $5^{\text {th }}$ and $95^{\text {th }}$ percentiles in the fibrillation histogram $\left(\mathrm{P}_{4, y_{5}}\right)$. The conduction velocity of uniformly propagating wavefronts during sinus rhythm, rapid pacing, and atrial fibrillation were calculated from the total conduction time across the mapping electrode. The average conduction velocity during atrial fibrillation in the free wall of the right atrium was measured as follows. During each beat, a conduction velocity map was calculated from the local activation times of four neighboring electrodes. The distribution of local conduction velocities was plotted in a histogram. The average of the median conduction velocities during a period of at least four seconds of fibrillation was used as a measure of average intra-atrial conduction velocities during atrial fibrillation.

\section{Statistical Analysis}

Results are expressed as average $5 \mathrm{SD}$. Bonferroni's modification of the t-test was used to compare differences between groups. The chi-square test was used to compare the characteristics of patients and the incidence of atrial fibrillation. Spearman's rank correlation test was used to test the correlation between median interval and variation of fibrillation with the degree of complexity of atrial activation. A value of $p<.05$ was considered statistically significant.

Rersutits

\section{Activation During Sinus Rhythm and Atrial Pacing}

Both during sinus thythm and during rapid atrial pacing ( 330 beats per minute), the free wall of the right atrium was activated uniformly by a single broad activation 

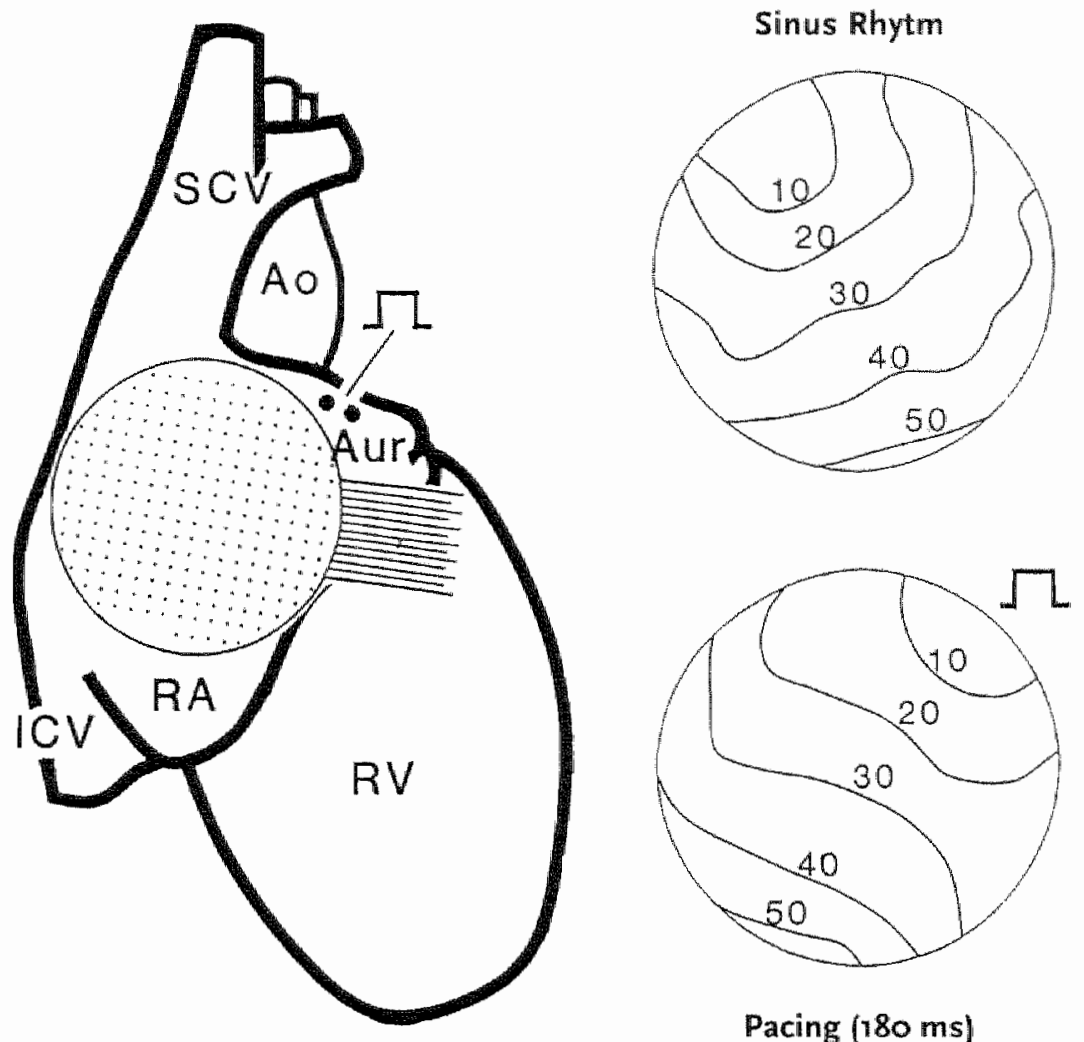

\section{Pacing (180 ms)}

Mapping of the free wall of the right atrium using a 244 lead epicardial mapping electrode (interelectrode distance, $2.25 \mathrm{~mm}$ ). Atrial pacing was performed through a separate pair of electrodes sutured to the right auricle (Aur). The electrograms recorded by 5 electrodes one in the center and four in the middle of each quadrant of the mapping area were used for making fibrillation interval histograms. Right panels, Activation maps during sinus ribythm and right atrial pacing (interval, 180 millseconds). No areas of slow conduction ar conduction block were present. 1 sochrones are drawn at 10 milisecond intervals. ao indicates aorta; $1 \mathrm{cw}$ inferior

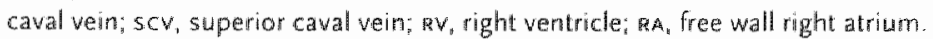



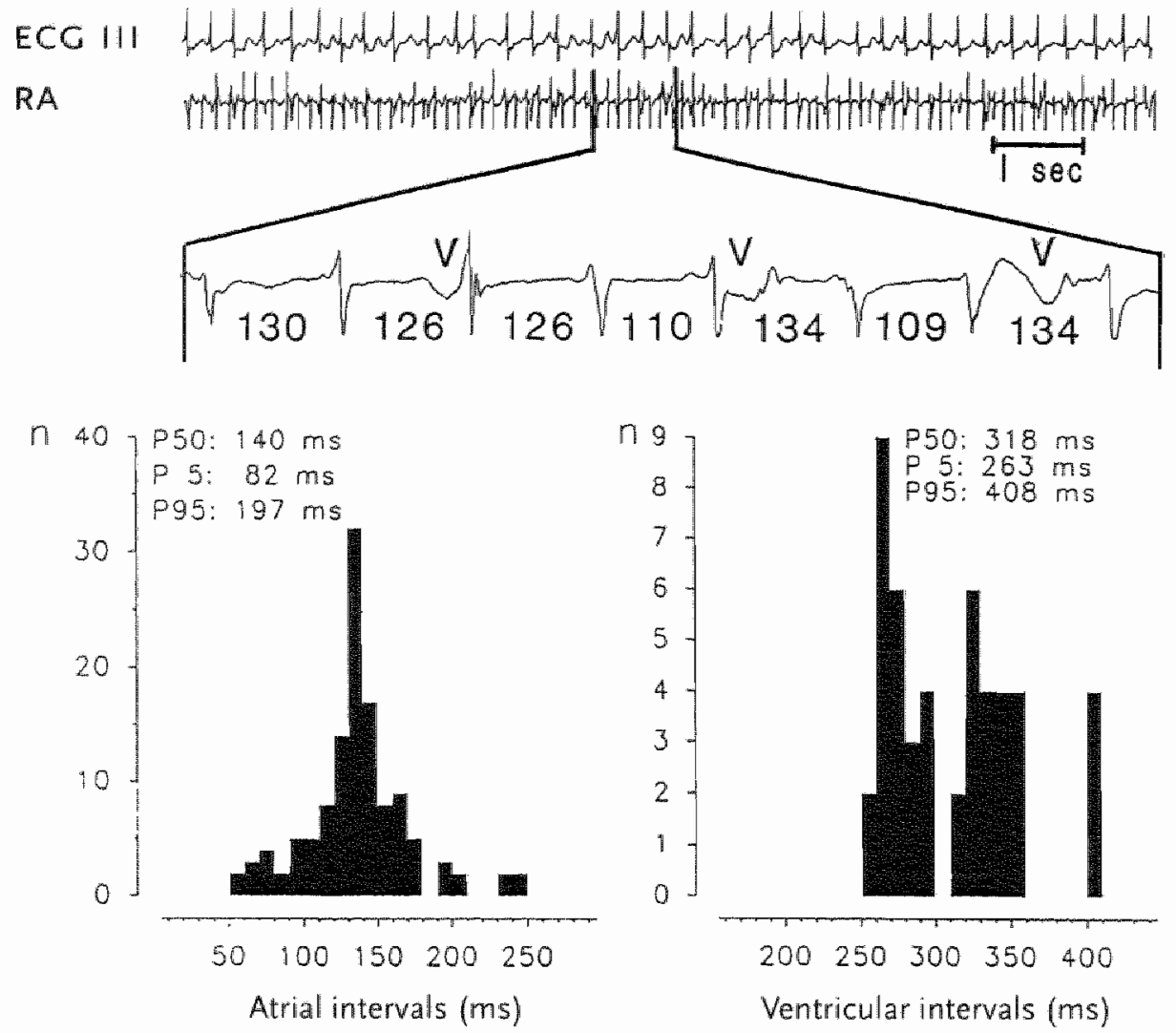

mgure 22

Ecc (lead $1 \mathrm{H}$ ) and a single unipolar right atrial electrogram (RA) during an episode of electrically induced atrial fibrillation lasting longer than two minutes (patient 12). Bottom, histograms are plotted of the atrial fibrilation intervals (left) and the RR intervals (right). The atrial fibrillation interval histogram was made from the intervals of 5 electrograms, reconded from the mapping area (see Figure 2.1). In this patient, the median fibrillation interval was 155 milliseconds $\left(P_{,}, 102\right.$ milliseconds; $P_{9 g^{\prime}}, 98$ milliseconds). The ventricular interval histogram showed a median walue of 301 milliseconds ( $p_{3,}, 256$ milliseconds: $p_{5, g^{\prime \prime}} 352$ milliseconds). $v$ indicates ventricular response. 
wave (Figure 2.1). No areas of slow conduction or conduction block were found. Activation of the right atrial free wall was always completed within 65 milliseconds, and only a minor beat-to-beat variation in the spread of activation was observed. The average conduction velocily during sinus rhythm was $73 \pm 5$ $\mathrm{cm} / \mathrm{s}(\mathrm{n}=25)$, and during rapid pacing $68 \pm 5 \mathrm{~cm} / \mathrm{s}(\mathrm{n}=18)(p=.002)$.

\section{Characteristics of Electrically Induced Atrial Fibrillation}

An example of electrically induced atrial fibrillation is given in Figure 2.2. The surface ECG showed an irregullar ventricular rhythm with pre-excited QRs-complexes without regular $\mathrm{p}$ - or $\mathrm{f}$-waves. The unipolar right atrial electrogram showed a rapid irregular thythm with a contimuous beat-to-beat variation in electrogran morphology and cycle length. The atrial fibrillation intervall histogram showed a consideralble variation in cycle length ( $\mathrm{P}_{5.95}, 96$ milliseconds) with a median value of 155 milliseconds. In this patient, the ventricular rhythm had a median interval of 301 milliseconds, varying from 256 milliseconds $\left(\mathrm{p}_{5}\right)$ to 352 milliseconds ( $\mathrm{p}$ ). In all patients $(n=25)$, the median fibrillation interval was $155+26$ milliseconds with a $p_{5.25}$ of intervals of $81 \pm 31$ milliseconds. The $k-n$ intervals varied between $249 \pm 81$ milliseconds ( $p_{5}$ ) and $468+67$ milliseconds ( $p_{95}$ ) (median, $379 \pm 5 n$ milliseconds).

\section{Mapping of Atrial Fibrillation}

In contrast to the uniform activation during sinus rhyth and atrial pacing, the free wall of the right atrium was during atrial fibrillation activated by more or less fragmented wavefronts. Although in all cases the right atrium was activated nonuniformly, the degree of fragmentation showed large inter-individual variations. In some patients, the right atrium was activated by single broad wavefronts exhibiting only a minimal degree of intra-atrial conduction block. In others. activation of the right atrium was highly fragmented by various arcs of intra-atrial conduction block dividing the depolarization wave into mulfiple wavelets.

In Figures 2.3 through 2.5, three cases of arral fibrillation are shown with an increasing degree of complexity in the pattern of activation. A relatively simple pattern of activation is illustrated in Figure 2.3 (patient 4). In this case, the median fibrillation interval was 174 milliseconds, with a $p_{\{, y s}$ of 38 milliseconds. During fibrillation, the right atrium was repeatedly activated by a single actiwation wave entering the mapping area from the right atrial appendage (at two oclock in the map). During sinus rhythm, the sinus impulse entered the mapping anea at 12 a'clock (not shown). Because of beat-to-beat variations in conduction velocity 


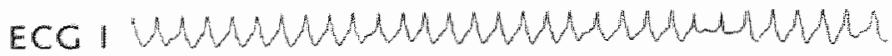
RA

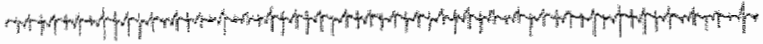
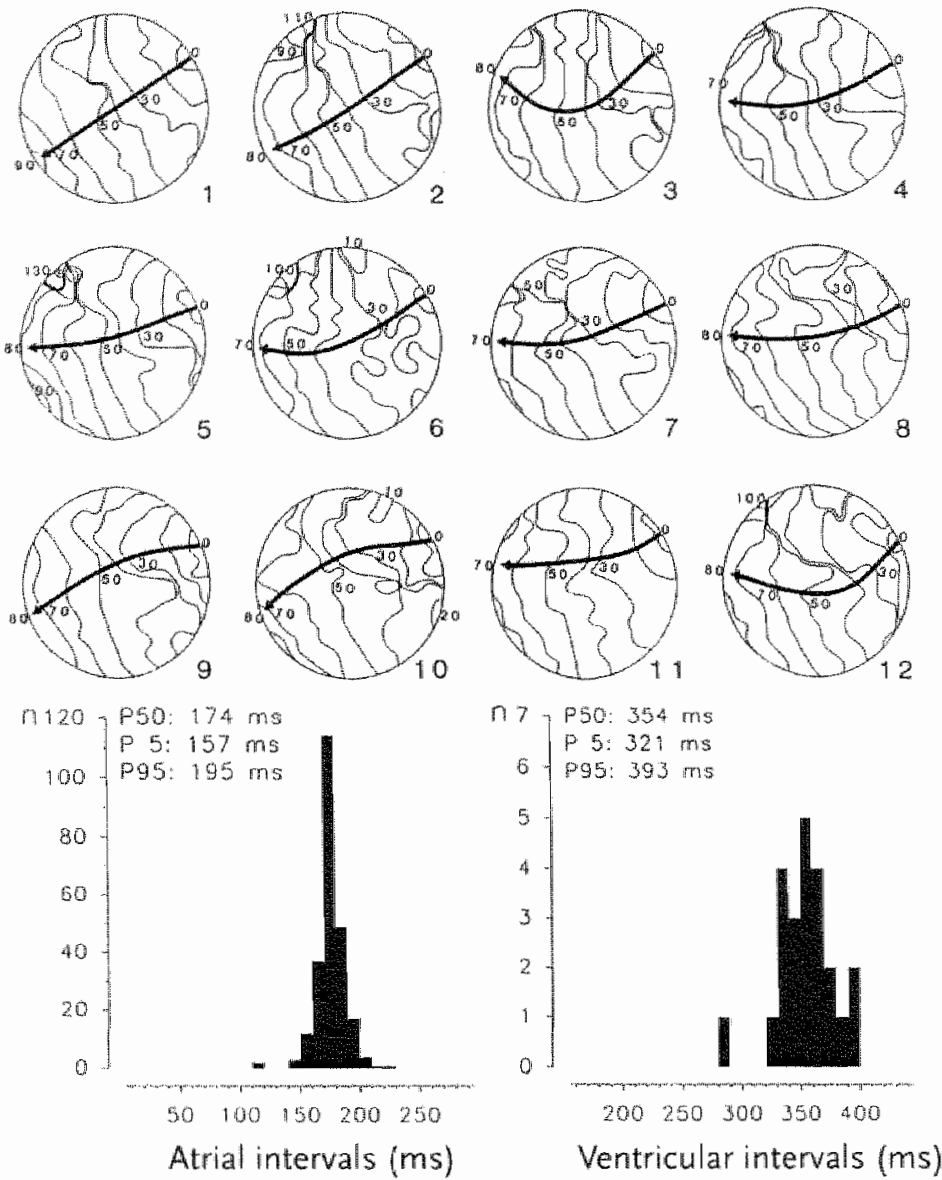

ligure 2.3 Ecc (lead 1), a right atrial unipolar electrogram (RA), activation maps of the free wall of the right atrium, and right atrial and wentricular interval histograms during atrial fibrillation (patient 4). Atrial fibrillation intervals in this patient ranged from 157 milliseconds ( $\left.p_{s}\right)$ to 195 milliseconds $\left(P_{95}\right)$, with a median value of 774 milliseconds. The median ventricular interval was 354 milliseconds ( $P_{y, g y}, 72$ milliseconds). The twelve activation maps of the free wall of the right atrium were taken at one-second interwals. Because the median fibrillation interval of this patient was 174 milliseconds, the maps of about one out of six beats are plotted. Isochrones have been drawn at 10 millisecond intervals. Thick lines represent arcs of conduction block. Arrows indicate the main directions of activation. In this case of atrial fibrillation, the right atrium was activated rather unformly. Single broad waves entered the Free wall from the atrial appendage and only small arcs of block were found. The total conduction time of the myocardium under the mapping electrode varied between 70 and 130 milliseconds. 
during fibrillation, the conduction time across the mapping area varied between 50 and 90 milliseconds, representing a conduction velocity between 40 and 72 $\mathrm{cm} / \mathrm{s}$. Only small arcs of intra-atrial conduction block were present (i.e., maps 2,4 through 7,10 , and 12). Because of delayed activation of areas distal to these arcs of conduction block, the total conduction time of the mapping area could be prolonged up to 130 milliseconds (beat 5).

In Figure 2.4, another case of atrial fibrillation is shown (patient 19), in which the median fibrillation interval was 147 milliseconds with a variation of 78 milliseconds $\left(p_{5,4.5}\right)$. In contrast to the case shown in Figure 2.3 , this patient showed a continuously changing pattern of excitation of the right atrium. The site of entrance of the fibrillation waves $(t=0)$ differed from beat-to-beat, whereas most of the time the right atrium was activated by two wavelets (maps 1, 3 through 5,7 and 11). During some beats, three different wavefronts could be iclentified (maps 10 and 12). Frequently long arcs of conduction block were present when depolarization waves encountered areas still refractory from activation by a previous wavelet. Also, many examples of collision or fusion of wavelets (maps 3 through 5,7 and 10 ) and local slowing of conduction (crowding of isochrones) can be seen (maps 2, 4 and 9). Only occasionally was the mapping area activated by a single depolarization wave (maps 6 and 8). In that case, the conduction time across the area was still relatively short ( 70 to 80 milliseconds) indicating that the intrinsic conduction properties of the atrium were not markedly depressed. The prolonged activation times of the right atrium were caused either by the turning of wavefronts around local arcs of conduction block (map 9) or by different wavelets entering the right atrium out of phase (map 1).

In Figure 2.5, the maps of atrial fibrillation are shown from a patient (3), in whom the activation of the right atrium was extremely complex. Also, during sinus rhythm or rapid pacing (interwal, 180 milliseconds), no signs of slow conduction or intraatrial conduction block were found. During atrial fibrillation, only one out of 12 maps showed a more or less uniform activation pattern with a conduction time across the mapping area of about 60 milliseconds (map 2). Thus, even in this case of highly complex atrial fibrillation, the intrinsic conduction properties of the atrium were still normal $(60 \mathrm{~cm} / \mathrm{s})$. Most of the time, the right atrium was activated by multiple narrow wavelets entering and leaving the mapping area at different sites. The conduction velocity of these wavelets varied considerably, as can be seen from the varying clistances between isochrones. Crowling of isochrones (conduction velocity $<10 \mathrm{~cm} / \mathrm{s}$ ), and multiple arcs of conduction block 


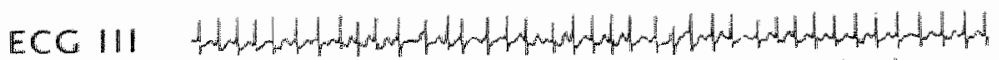
RA

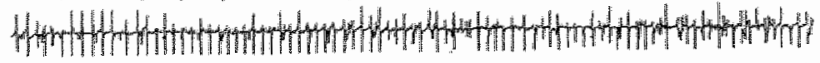
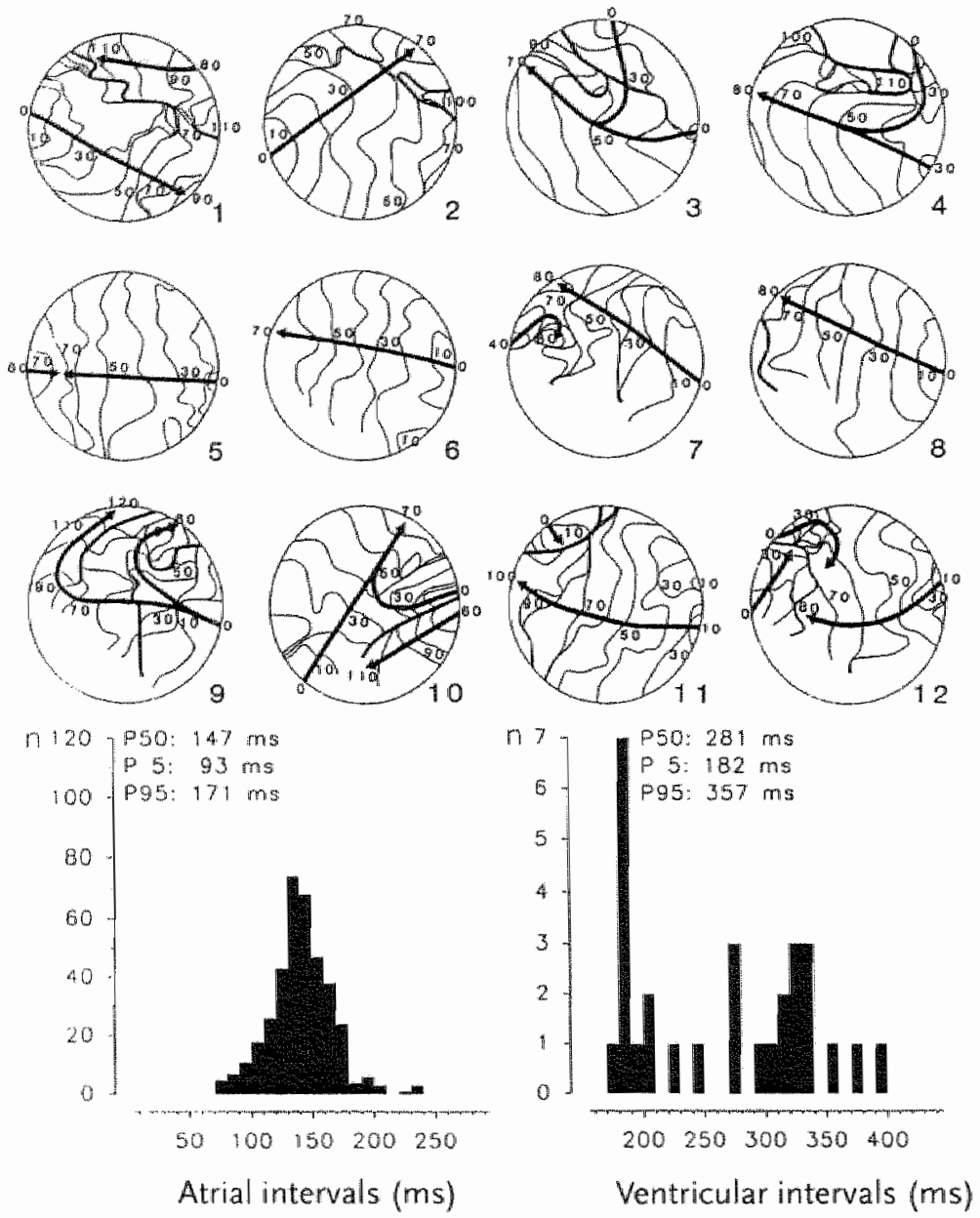

Figure 2.4

Ecc (lead iI), a right atrial unipolar electrogram (RA), activation maps of the right atrial free wall, and a right atrial and ventricular interval histogram during atrial fibrillation (patient 19 ). Atrial fibrillation interwals in this patient ranged from 93 milliseconds ( $p$ ) to 171 milliseconds $(F)$. with a median value of 147 milliseconds. The median ventricular interval was 287 milliseconds ( $p_{5,35}, 75$ milliseconds). The twelve actiwation maps were taken at one-second intervals. Compared with the activation patterns in Figure 2.3, in this case, the actiwation of the right atriurr was more complex. During most beats, two wavelets entered the mapping area from different directions. Frequently, areas of slow conduction, as indicated by crowding of isochrones, were present. Only incidentally was the right atrium activated by a single wavefront conducting at high speed (map 6). 


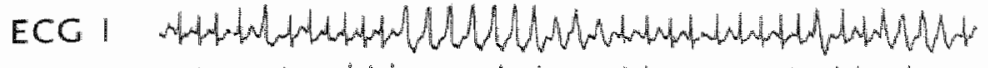
RA
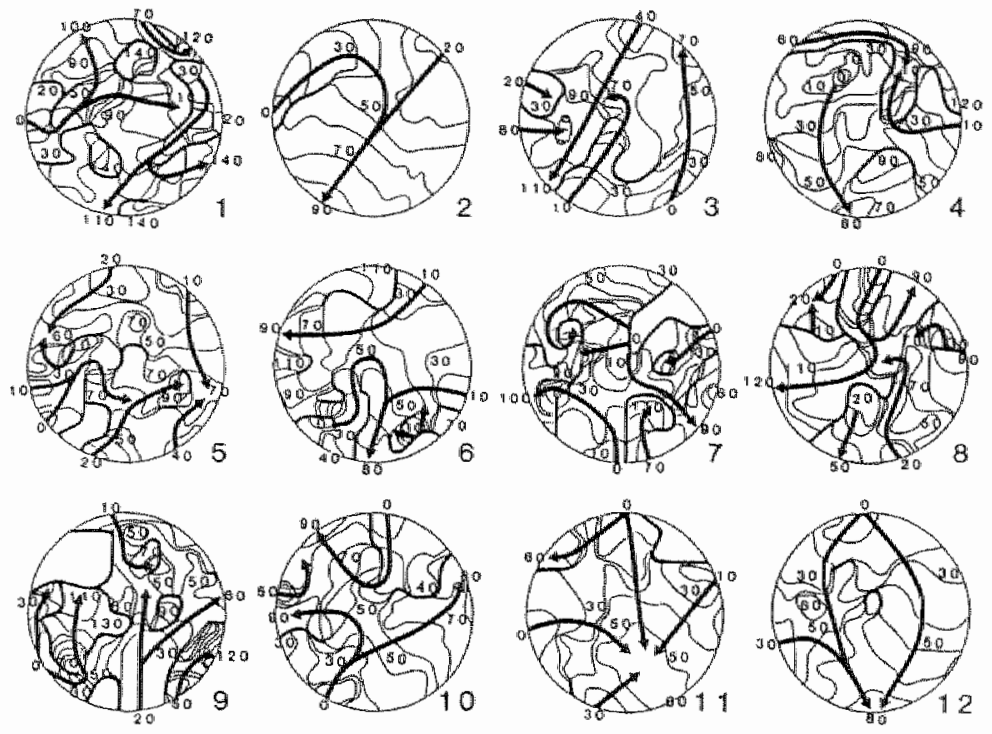

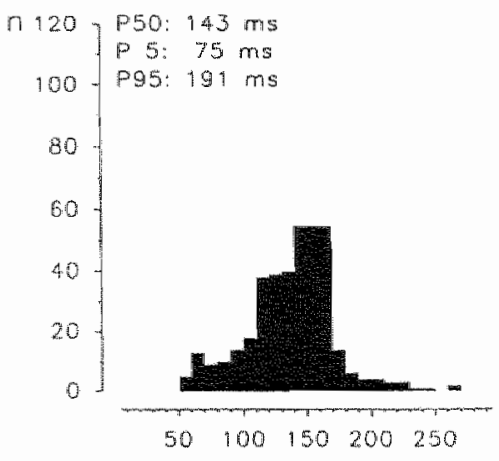

Atrial intervals (ms)

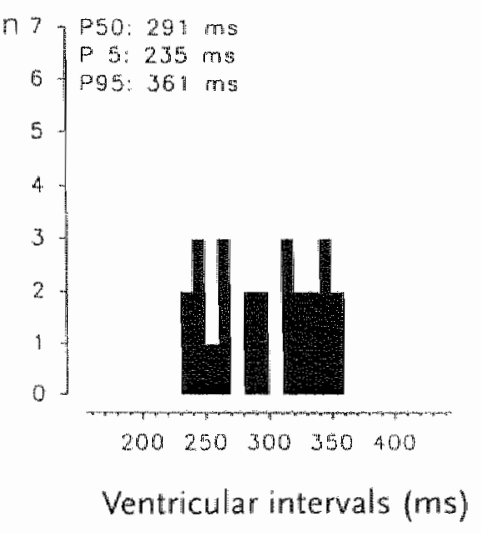

Ecs (lead i), a right atrial unipolar electrogram (na), activation maps of the right atrial free Figure 2.5 wall, and a right atrial and ventricular interval histogram diuring atrial fibrillation in patient 3 . The warying morphology of the QRS complex is caused by a varying degree of premexcitation. The activation maps were taken at one-second intervals. In this case, the right atrium showed highly fragmented activation by multiple wavelets. 
can be found in almost every map. After collision and fusion of wavelets, branching of a wavefront into wo separate wavelets occurred most frequently (maps 1 and 7 through 11 ). Often, areas which had been activated by one of these wavelets where reexcited by another wavelet (random reentry). In this patient, the fibrillation intervals varied widely between 75 milliseconds $\left(\mathrm{s}_{\mathrm{s}}\right)$ and 191 milliseconds $\left(p_{\text {g }}\right)$ with a median interval of 143 milliseconds.

\section{Continuous Electrical Activity}

In Figure 2.6, the percentage of the mapping area activated during atrial fibrillation for the three patients shown in Figures 2.3 through 2,5 , is shown for ach 10 millisecond time window. These graphs express the temporal variation of right atrial tissue mass that is being depolarized during fibrillation. In patient 4 (top), electrical activation of the right atrium clearly alternated with periods during which no depolarization waves were present under the mapping electrode $139 \%$ of the time). In patient 19 (middle), no electrical activity was recorded during $23 \%$ of the time, whereas in patient 3 (bottom) the right atrium was free of depolarization waves only $7 \%$ of the time. In this patient, during atrial fibrillation long episodes of continuous electrical activity were recorded from the right atrium. One of these episodes of continuous electrical activity is analyzed in Figure 2.7. At the top, the main trajectory of the fibrillation waves is plotted, and at the bottom, the individual isochrone maps are given. In the first map, the right atrium was activated by a single wave entering the mapping area from below and leaving it at the top at $t=1.40$ milliseconds. While this activation wave was still present, a new wave entered the mapping area at $t=120$ milliseconds (map 2). As can be seen from the trajectory diagram, this wave started to circulate in the mapping area for a period of longer than one second. Maps 2 through yo show the details of this continuously shifting leading circuit. Frequenty offsprings from this wandering circuit propagated away from the mapping area towards the temainder of the atria. At the same time, other fibrillation waves entered the mapping area and fused with the wandering leading circuit. At $t=1200$ milliseconds, the circulating wavefront was blocked (double bar) and the circuit was interrupted. However continuous electical activity persisted because in the meartime ( 1130 milliseconds) another wavelet had already entered the mapping area. Thus, continuous electrical activity during atrial fibrillation was based on wo mechanisms: (1) the presence of a continuously reentering impulse (shifting leading circuit), and (2) temporal overlap of different waxes entering the mapping area at different times. 


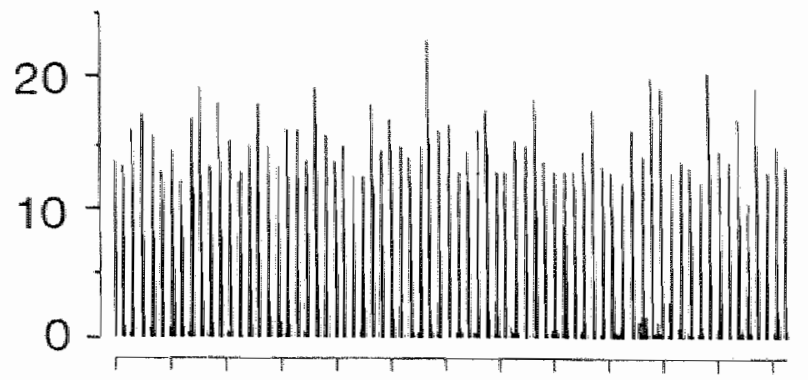

$39 \%$
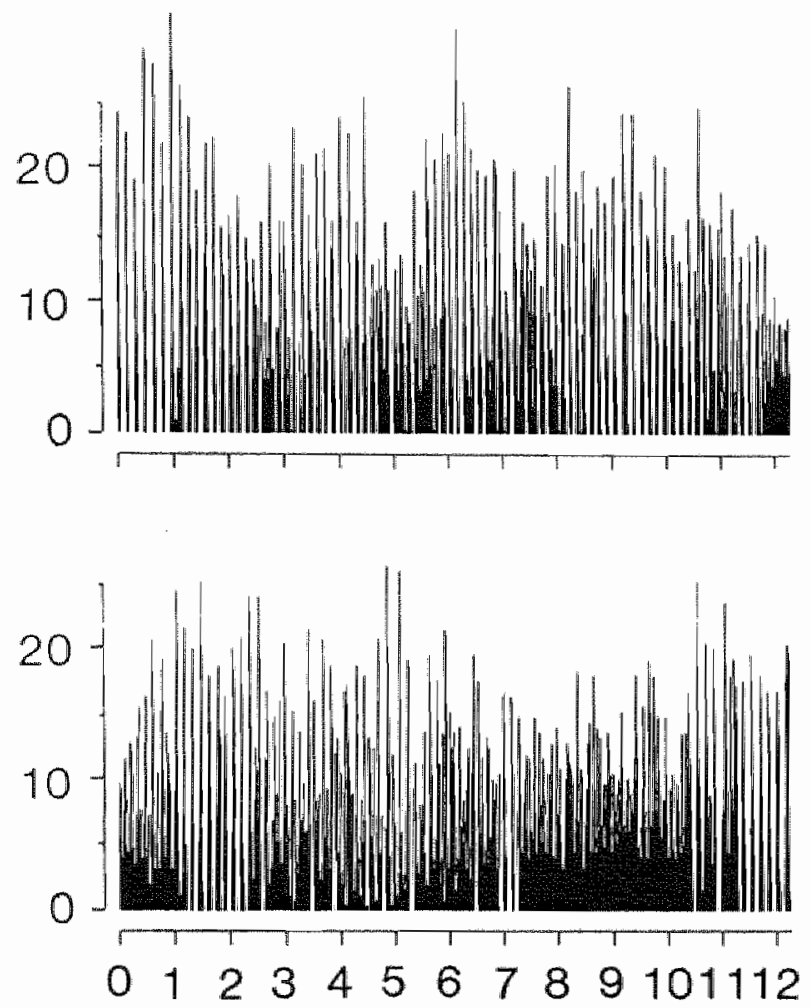

Time (s)

Temporal variation in right atrial tissue mass activated during atrial fibrillation in the three patients shown in Figures 2.3 through 2.5 . In each diagram, the percentage of the mapping area (ordinatte) depolarized during each isochrone of 10 milliseconds is plotted sequentially (abscissa). Going from the simplest case of atrial fibrillation (top) to a more complex one (bottom), the time that no electrical activity was present in the free wall of the right atrium decreased from $39 \%$ to $7 \%$. During complex fibrillation, frequently periods of continuous electrical activity occurred lasting several seconds. 


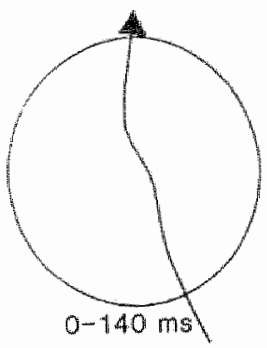

Trajectory
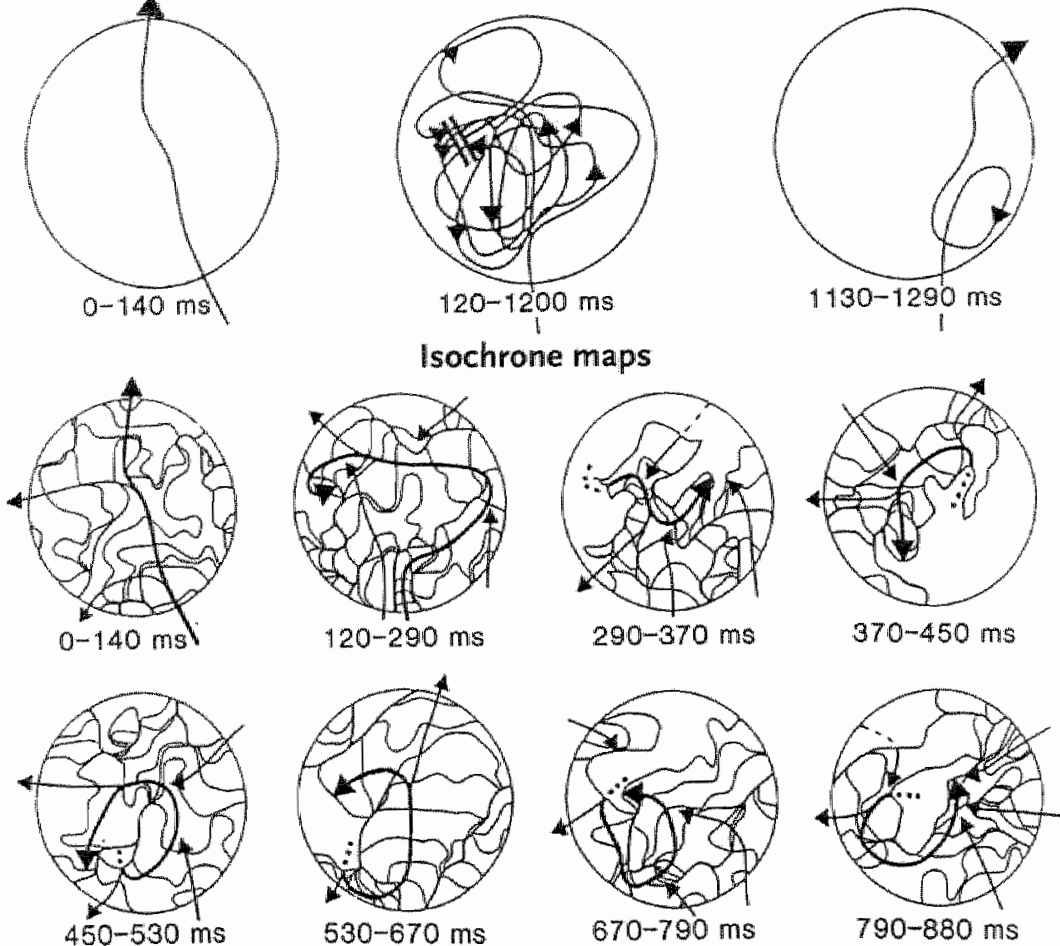

$530-670 \mathrm{~ms}$
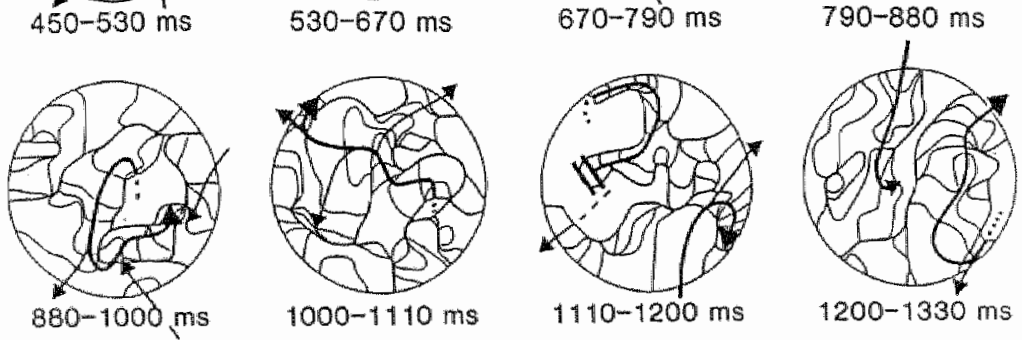

Figure 2.7 Right atrial activation during a period of continuous electrical activity (patient 3). The diagrams at the top show the trajectory of the main fibrillatory wavefronts. Below the corresponding isochrone maps during this period of continuous electrical activity are given. The first trajectory and isochrone map show a single wave activating the mapping area from $l=0$ to thm 40 milliseconds. While this activation wave was still present, another wave entered the mapping area at $t=120$ milliseconds. As can be seen from the second trajectory diagram and isochrone maps 2 through 10 , this wave started to circulate in the mapping area for more than ome second. At t= 200 this wave was blocked (double bar) and the wandering circuit was interrupted (map 11). However, continuous electrical activity persisted since at the same time (t=n130), another wave entered the mapping area (right trajectory and maps 11 and 12). 


\section{Classification of Atrial Fibrillation}

In an attempt to quantify the degree of complexity of atrial activation during fibrillation, all maps ( $n=1500$ ) ware classified into three categories. The criter ia used for classification were as follows (Figure 2.8).

\section{Type I}

Single broad wavefronts propagating without significant conduction delay, exhibiting only short ares of conduction block or small areas of slow conduction not disturbing the main course of propagation, were considered to describe type $\mathrm{t}$.

\section{Type II}

Activation patterns characterized either by single waves associated with considerable amount of conduction block and/or slow conduction or the presence of two wavelets, were considered to describe type il.

\section{Type III}

The presence of three or more wawelets associated with areas of slow conduction ( <10 $\mathrm{cm} / \mathrm{s}$ ) and multiple arcs of conduction block, were considered to describe type nif.

\section{Three types of atrial fibrillation}
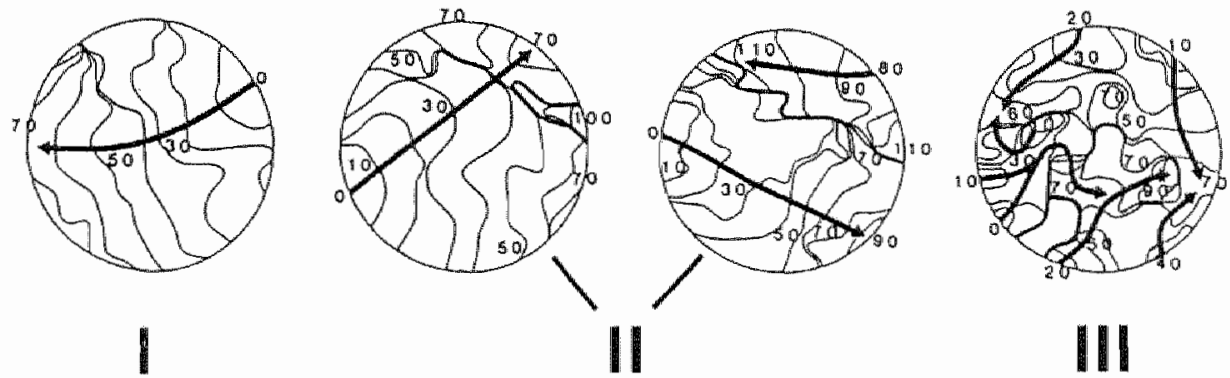

II

III

Mapping criteria for cllassification of atrial fibrillation. Type lis characterized by single, uniformly propagating waves. During type 11 , single non-uniformly conducting waves or two wavelets are present. Type 111 is characterized by the presence of three or more wavelets. associated with multiple areas of slow conduction and arcs of conduction block. 
In Table 2.2, the 25 patients are classified according to the degree of complexity of atrial fibrillation. An incidence of more than $50 \%$ of type 1 or type m beats was chosen as the boundary between the types of fibrillation. In this way, ten patients $(40 \%)$ were classified as having type $i_{3}$ eight patients $(32 \%)$ as having type II and severi patients (28\%), as having type ri fibrillation.

No statistical significant difference was found among the three groups of patients with respect to age $(p>.897)$, sex $(p>477)$, location of the accessory pathwayl $s$ ) $(p>199)$, atrial refractory period $(P>859)$, left atrial size $(p>.455)$, incidence of documented atrial fibrillation $(P>165$ ), and duration of electrically induced atrial. fibrillation $(p>.184$ ). However, because of the small sample size and the large variation of the various parameters in each group, the confidence limits were rather wide and consequently the ability to discern differences was limited. Thus, in this case, the absence of statistical. significance does not necessarily mean that no clinical differences exist between patients with different types of atrial fibrillation.

The conduction properties of the right atrium during sinus rhythm $(p=.371)$, rapid atrial pacing $(p=.478)$, and uniform propagation of fibrillation waves $(p=.38 \mathrm{~s})$ did not differ in patients with types 1 , rr or ruf fibrillation (Table 2.2). Because the standard deviations of these parameters were small, the confidence limits were quite narrow, and in this case, the statistical analysis indicates a true absence of differences between the groups.

Compared to sinus rhythm, during atrial fibrillation intra-atrial conduction was clearly depressed $(53 \pm 12 \mathrm{~cm} / \mathrm{s}$ compared with $73 \pm 5 \mathrm{~cm} / \mathrm{s})$. In patients with type I fibrillation, the average median conduction velocity ( $\mathrm{F}_{\mathrm{awg}}$ ) was still relatively high $(61 \pm 6 \mathrm{~cm} / \mathrm{s})$. During type in fibrillation, average conduction velocity was $54 \pm 4$ $\mathrm{cm} / \mathrm{s}(p<.02)$, whereas during type un fibrillation, conduction velocity was decreased to $38 \pm 10 \mathrm{~cm} / \mathrm{s}(\mu<.001)$.

The type of fibrillation was correlated to the fibrillation interwal. In type 1 , the average median interval was $174 \pm 28$ milliseconds, whereas in types il and 11, the median fibrillation intervals were $150 \pm 14$ and $136 \pm 16$ malliseconds $(P<.05)$. When the median fibrillation interval was shorter, the wariation in fibrillation intervals was larger. In type I fibrillation, the $P_{5,95}$ of the fibrillation intervals was $54 \pm 25$ milliseconds, whereas in lypes 11 and $u$ these values were 94121 and 104122 milliseconds, respectively $(p<.005)$.

Also, the time during which no propagating waves were present in the mapping area became shorter with increasing complexity of fibrillation. In typer, most beats 
were separated by a clear period of electrical mactivity and during about half of

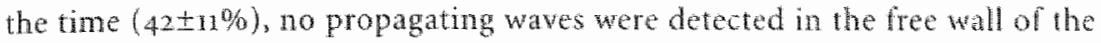
right atrium. Electrical inactivity was still frequently observed in type u fibrillation $(21+4 \% ; P<.001)$, although short periods of continuous clectical activity also were observed. In contrast, during type m fubrillation, episodes of continuots right atrial activity were predomimant, and propagating wavklets were absent during only $8 \pm 4 \%$ of the time $(p<.001)$.

Athough the above classiffcation of atrial fibrillation may be useful, we want to emphasize that the three types of fibrillation stould not be regarded as separate entities. The different types of fibrillation should rather be regarded as part of a continuous spectrum of increasing complexity. This is illustrated in Figure 2.9. in which the 25 patients ane ranked according to the degree of complexity of atrial fibrilation. On the ordinate, the median fibrillation interval and the wariation in fibrillation interval $\left(\mathrm{p}_{5}\right.$ ) $)$ are plotted. As can be seen, going from the simplest type I to the most complex type ur patient, the median interval gradually shortens from 212 to 120 milliseconds, whereas the $P_{5}$ increases from 21 to 123 milliseconds. No clear separation exists between the different rypes of attial Gibrillation.

\section{Differences Between Right and Left Atrium}

In eight patients, we mapped the free wall of the left atrium simulaneously during the same episode of atrial tibrillation. In Table 2.3, the type of fibrillation in each atrium is compared. In five of eight patients, the right and left atrial free walls showed the same type of atrial fibrillation. In two patients, the left atrium showed a more disorganized activation pattern, whereas in one patient, the left alrum was more organized than the right. No differences in fibrillation interval or wariation in fibrillation intervals $\left(\mathrm{P}_{5}, \mathrm{se}_{\mathrm{s}}\right)$ were found between the right and the keft atrat $(P>492)$.

\section{Incidence of Reentry}

During atrial fibrillation, wo different kinds of reentrant excitation were observed in the free wall of the right atrium. One type of reentry (random reenury) was defined by Hoffman and Rosen"s. as "depending on multiple areas in wich the impulse blocks in a undirectional manner lalthough the sites of block change from moment to moment and on the continuous propagation of the impulse [over random paths] so that individual groups of fibers are excited repetitively." 


\section{Table 2.2 Classification of Atrial Fibrillation}

\begin{tabular}{|c|c|c|c|c|c|c|c|c|}
\hline \multirow[b]{2}{*}{ Tryotang } & \multirow[b]{2}{*}{ 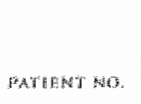 } & \multicolumn{3}{|c|}{ 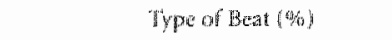 } & \multicolumn{4}{|c|}{ Conduction Velocity (cms) } \\
\hline & & : & и & m & sk & PACTMG & AFuni & Afarg \\
\hline \multirow[t]{10}{*}{$r$} & 24 & 1100 & 0 & 0 & 76 & 73 & 71 & 69 \\
\hline & 8 & 100 & 0 & 0 & 75 & $=$ & 72 & 70 \\
\hline & 21 & 100 & 0 & 0 & 73 & 70 & 60 & 59 \\
\hline & 17 & 100 & 0 & 0 & 62 & - & 51 & 51 \\
\hline & 4 & 96 & 4 & 0 & 79 & 69 & 65 & 56 \\
\hline & 14 & 93 & 7 & 0 & 76 & 65 & 61 & 59 \\
\hline & 10 & 91 & 9 & 0 & 76 & - & 62 & 55 \\
\hline & 11 & 90 & 10 & 0 & 77 & $7 \|$ & 69 & 66 \\
\hline & 15 & 79 & 21 & 0 & 76 & 69 & 66 & 62 \\
\hline & 9 & 84 & 10 & 6 & 78 & 69 & 68 & 65 \\
\hline Avg 150 & & $93 \pm 7$ & $6 \pm 7$ & $1 \pm 2$ & $75 \div 5$ & $69 \pm 3$ & $64 \pm 6$ & $61 \pm 6^{*}$ \\
\hline \multirow[t]{8}{*}{ il } & 5 & 27 & 69 & 4 & 66 & 65 & 60 & 57 \\
\hline & 19 & 33 & 50 & 17 & 78 & 73 & 54 & 50 \\
\hline & 12 & 31 & 49 & 20 & 63 & - & 55 & 51 \\
\hline & 7 & 20 & 68 & 12 & 75 & 70 & 69 & 59 \\
\hline & 6 & 30 & 33 & 31 & 71 & 66 & 63 & 50 \\
\hline & 23 & 0 & 89 & : & 79 & - & 70 & 58 \\
\hline & 25 & 0 & 65 & 35 & 70 & 66 & 60 & 53 \\
\hline & 20 & 0 & 56 & 44 & 77 & $\cdots$ & 64 & 56 \\
\hline Avgetso & & $18 \pm 16$ & $60 \pm 17$ & $22 \pm 14$ & $72 \pm 6$ & $68 \pm 3$ & $62 \pm 6$ & $54+44^{*}$ \\
\hline \multirow[t]{7}{*}{ nir } & 18 & 3 & 28 & 69 & 75 & 71 & 60 & 53 \\
\hline & 3 & 6 & 10 & 84 & 78 & 68 & 64 & 32 \\
\hline & 2 & 2 & 8 & 90 & 74 & 70 & 55 & 50 \\
\hline & 13 & 0 & 6 & 94 & 70 & - & 53 & 35 \\
\hline & 16 & 0 & 5 & 95 & 66 & 62 & $6 i$ & 38 \\
\hline & 22 & 0 & 4 & 96 & 69 & 63 & 66 & 34 \\
\hline & 1 & 0 & $i$ & 99 & 72 & 66 & 60 & 25 \\
\hline Avgtso & & $2 \pm 2$ & $9 \pm 9$ & $90 \pm 10$ & $72 \pm 4$ & $67 \pm 4$ & $60 \pm 5$ & $38 \pm 10^{*}$ \\
\hline \multicolumn{5}{|c|}{ Total Akgtso } & $73 \pm 5$ & $68 \pm 3$ & $62 \pm 6$ & $53 \pm 12$ \\
\hline
\end{tabular}

SR indicates sinus thythm; ar, atrial fibritlation: Aftini, conduction velocity of uniform beats during atrial fibrillation; Afavg. average median conduction velocity during atrial fibrillation; ", p<., o5 compared with

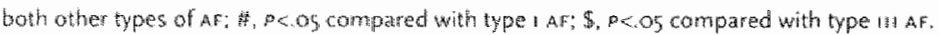




\begin{tabular}{|c|c|c|c|c|c|c|}
\hline \multirow[b]{2}{*}{ WATEENT NO } & \multicolumn{2}{|c|}{ Interval (ns) } & \multirow{2}{*}{ 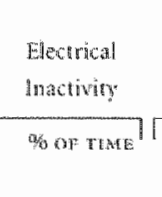 } & \multicolumn{2}{|c|}{$\begin{array}{c}\text { Inciderwe of kent } y^{2} \\
\text { (os of beass) }\end{array}$} & \multirow{2}{*}{ 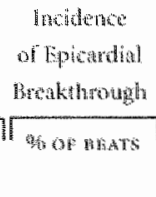 } \\
\hline & MEDLAN & $y^{\mathbf{z}}$ & & Renom & $\begin{array}{l}\text { HABDING } \\
\text { CINethe }\end{array}$ & \\
\hline 24 & 212 & 21 & 60 & 0 & 0 & 9 \\
\hline 8 & 204 & 19 & 55 & 0 & 0 & 0 \\
\hline 21 & .193 & 42 & 32 & 0 & 0 & 0 \\
\hline 17 & 184 & 80 & 42 & 0 & 0 & 0 \\
\hline 4 & 174 & 38 & 39 & 0 & 0 & 0 \\
\hline 14 & 155 & 79 & 35 & 0 & 28 & 0 \\
\hline 10 & 132 & 46 & 24 & 0 & 13 & 10 \\
\hline 11 & 150 & 59 & 45 & 0 & 0 & 0 \\
\hline 15 & 140 & 87 & 39 & 0 & 0 & 0 \\
\hline 9 & 191 & 69 & 52 & 0 & 0 & 0 \\
\hline & $\pm 28^{*}$ & $54+25^{*}$ & $42 \pm 11^{*}$ & $0 \pm 0$ & $3+7^{2}$ & $0 \pm 04$ \\
\hline
\end{tabular}

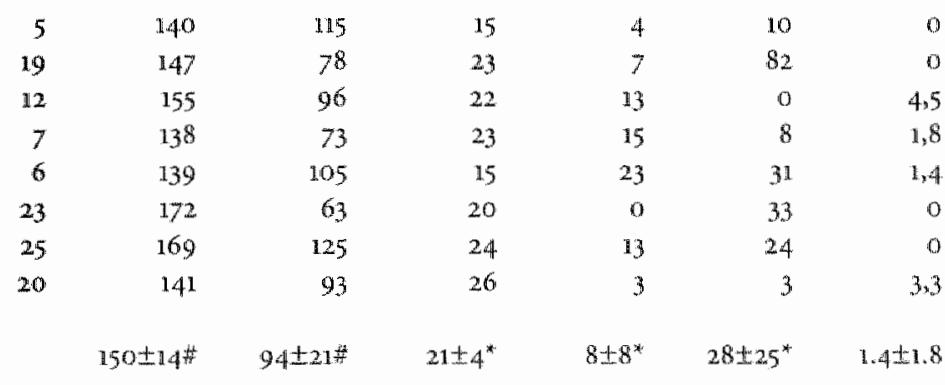

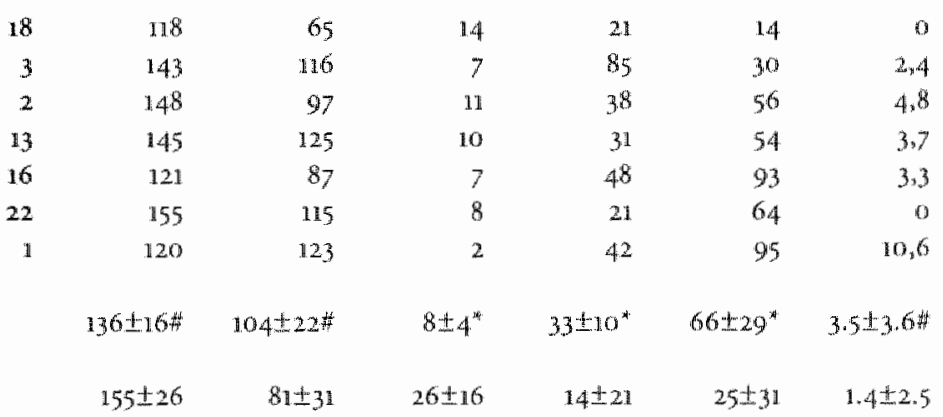


Table 2.3 Comparison of Right and Left Atria

\begin{tabular}{|c|c|c|c|c|c|c|}
\hline \multirow[b]{3}{*}{ PATHET KNO } & \multicolumn{3}{|c|}{ Ragh Atrium } & \multicolumn{3}{|c|}{ Lef Arōtmes } \\
\hline & \multirow{2}{*}{ TYPE GE AF } & \multicolumn{2}{|c|}{ Inerwall (mis) } & \multirow[b]{2}{*}{ ThPe of A } & \multicolumn{2}{|c|}{ interyal thot } \\
\hline & & MEBAR & $P_{5}-95$ & & Medman & $p_{5}, 95$ \\
\hline 4 & $\mathbb{1}$ & 174 & 38 & 1 & 197 & 84 \\
\hline 14 & I & 155 & 79 & $I$ & 167 & 24 \\
\hline 9 & 1 & 191 & 69 & H & 167 & 92 \\
\hline 12 & II & 155 & 96 & II & 150 & 109 \\
\hline 6 & $\|$ & 139 & 105 & III & 133 & 126 \\
\hline 25 & II & 169 & 125 & a & 156 & 107 \\
\hline 2 & III & 1,48 & 97 & II & 154 & 112 \\
\hline 16 & III & 121 & 87 & III & 143 & 126 \\
\hline Aggtsu & & $57 \pm 22$ & $87 \pm 26$ & & $158 \pm 19$ & $98 \pm 13$ \\
\hline
\end{tabular}

Af indicates atria fibrillation

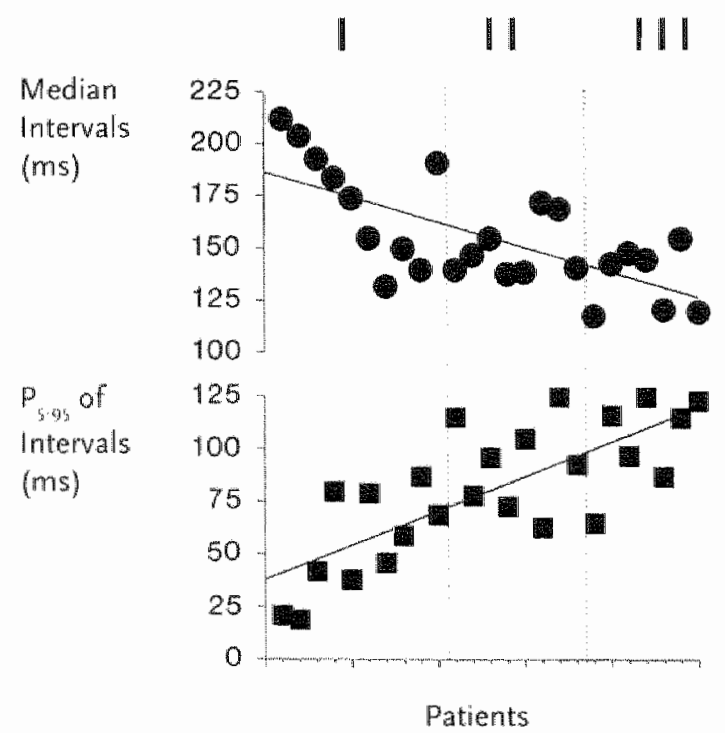

Figure 2.9 Three lypes of atrial fibrillation as a continuous spectrum of atrial fibrillation. On the abscissa, the 25 patients are ranked in order of increasing complexity of their activation patterns of atrial fibrillation. On the ordinate, the median fibrilation interval and the variation in fibrillation interval $\left(p_{5, g}\right)$ are plotted. Going from the most simple case (type 1$)$ to type 11 fibrillation, both the interval and the variation in fibrillation interwals changed progressively. There were no abrupt changes in these variables between the different types of fibrillation. 
We narrowed the definition of random reentry to the phenomenon of an propagating wavelet re-excing an area that shorty before liad been activated by another present wavelet. An example of random reentry is given in Figure 2.10 (left panels). In the apper map, a fibrillatory wavellet is entering the mapping area from the left ( $t=0$ ) activating electrodes 1,2 and 3 , in that order. This wavelet was blocked between electrodes 3 and 4 . Later ( $t=70$ milliseconds), another wavelet entered the mapping area at electrode 6 and propagated all the way from right to left from electrodes 6 to 1 . Thus, sites 3 to 1 , which were previously activated from left to right, were now reentered by the second wave from right to left. As a result, at the site of unidirectional block, a short interval of 76 milliseconds was found that gradually became longer at more distant electrodes ( 105 and 136 milliseconds at electrodes 2 and 1). An example of the other type of reentry is illustrated in the middle panels of Figure 2.10 (leading circle reentry). In this case the impulse circulated in a clockwise direction from electrodes 110 around a central arc of functional conduction block of about two cm long. While the revolution time of the first beat was 138 milliseconds, during the next beat (lower map) the central arc of conduction block shifted slightly, resulting in a revolution time of 127 milliseconds. Fixed reentrant circuits were never observed in the free wall of the right atrium.

The incidence of both random reentry and leading circle reentry were different in the three types of fibrillation (Table 2.2). In type I fibrilation, random reentry was not observed, and leading circle reentry occurred in only two patients. The number of beats that a leading circle persisted in the free wall of the right atrium was small ( $2.4 \pm 1.4$ beats). In type rl, random reentry occurred in $8 \pm 8 \%$, and also shifing leading circles were more common ( 28 土25\%). In type $1 \mathrm{~m}$, randon reentry occurred in $33 \pm 10 \%$ of the beats, whereas a shifting leading circle was observed during $66 \pm 29 \%$ of the fobrillation cycles. During type un, the average persistence of a shifing leading circle in the free wall of the right atrium was $5.4+3.4$ beats. The incidence of both shitting circles and random reentry was significantly different in all groups $\left(p \alpha_{\text {. }} 005\right)$.

\section{Epicardial Breakthrough}

Incidentally a 'new' wavelet appeared to originate from somewhere in the free wall of the right atrium. The right panels in Figure 2.10 give an example of this phenomenon. The upper map shows a focal pattern of activation starting at the site indicated by the asterisk. From the mapping data, no evidence could be 


\section{Random Reentry}
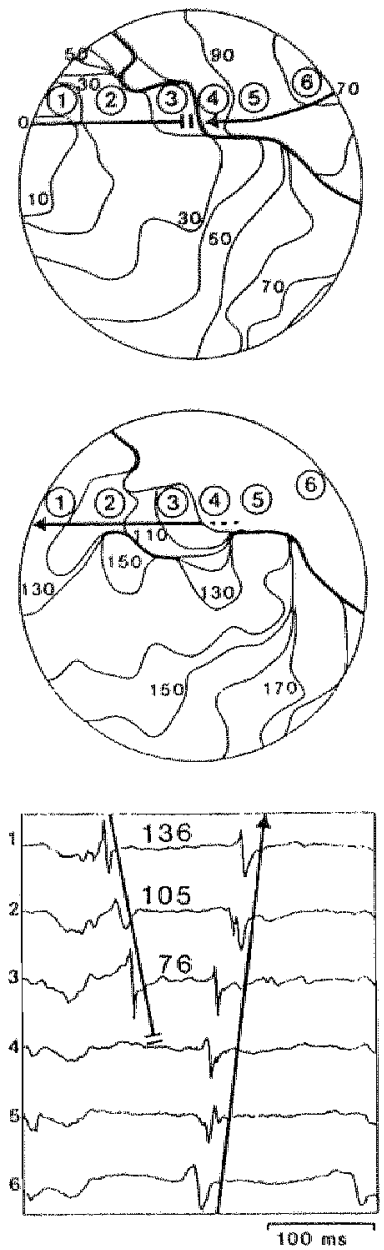

Leading Circle Reentry
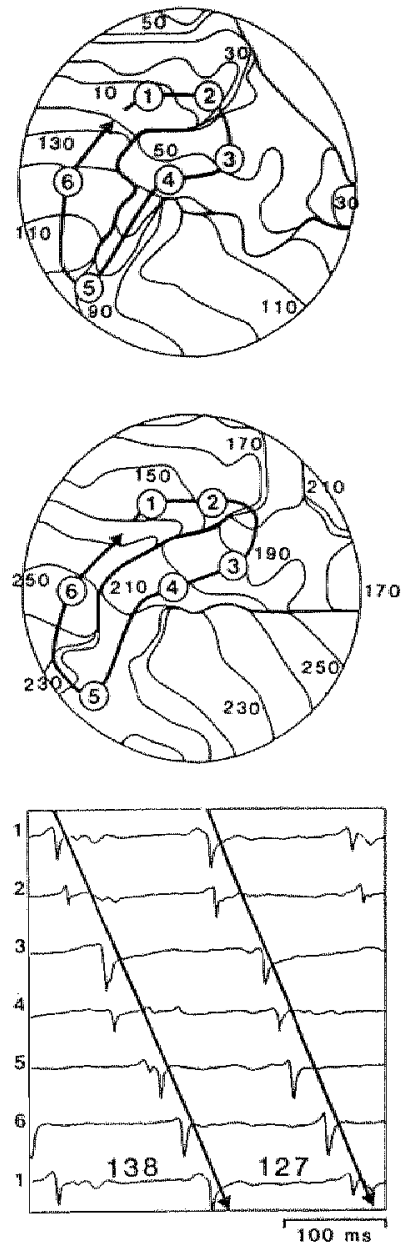

\section{Epicardial Breakthrough}
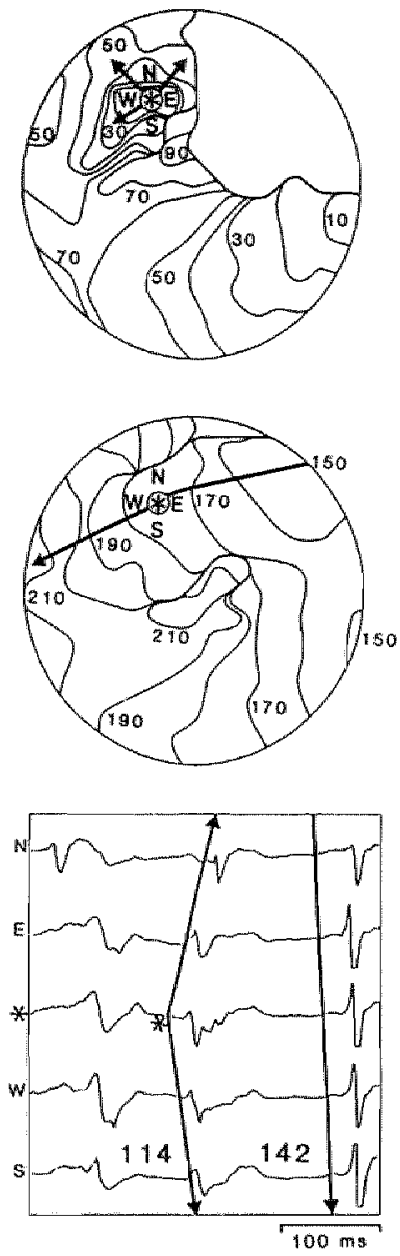

Figure 2.10

Two different types of reentry (random reentry and leading circle reentry) and a focal type of activation during atrial fibrillation. The sites of the unipolar electrograms given below the maps are indicated on the maps. During random reentry, a wavelet reexcites tissue which shortly before has been activated by another simultaneously present wawelet. During leading circle reentry, the impulse circulates around a shifting central line of functional conduction block. A focal pattern (right) was preceded by a small $\mathbb{R}$ wave at the earliest electrogram (*) and was never observed during more than one beat. See text for discussion. 
obtaned for epicardial propagation towards this earliest site of activation. It seems unlikely that these focal patterns of activation originated from the simus node because during nomal sinus rhythm, the sinus impulses always originated at a point outside the mapping area. Although abnormal automaticity camnot be completely excluded, the most likely explanation for this excitation pattern is epicardial breakthrough of a fibrillation wave propagating in a free running endocardial trabecula. ${ }^{60}$ In agreement with this explanation is the fact that the electrograms at the earliest sites of activation usually exhibited a small i wave before the intrinsic negative deflection. In addition, epicardial breakthrough only occurred at different sites as solitary events. Repecitive focall responses were never observed. In Table 2.2, the incidence of epicardial breakthrough is given for the three types of atrial fibrillation. In type 1 , a focal pattern was never seen. Also, in types $u$ and $\mu$, epicardial breakthrough was a rare event, occurring in $1.4 \pm 1.8 \%$ and $3.5 \pm 3.6 \%$ of the beats, respectively.

\section{Discussion}

\section{Classification of Atrial Fibrillation}

Based on the morphology of a single bipolar atrial electrogram (electrode distance 0.5 $101.0 \mathrm{~cm}$ ) Wells et al. ${ }^{72}$ distinguished four types of atrial fibrillation. In type 1 fibrillation, the electrogram showed discrete complexes of variable morphology separated by a clear isoelectric baseline. Type u fibrillation was also characterized by discrete atrial beat-to-beat complexes of variable morphology but differed from type $\mathrm{i}$ in that the baseline showed continuous perturbations of varying degrees. During type in fibrillation highly fragmented atrial electrograms were recorded that showed no discrete complexes or isoelectric intervals. Type iv fibrillation was characterized by alternation between types in and the other types. In our opinion this should not be regarded as a separate type of fibrillation but rather indicates the general property of atrial fibrillation to exhibit temporal variations both in rate and irregularity.

Although we used the same terminology for classification as Wells et al. ${ }^{2}$ (i.e., types 1 , In, and In), our criteria for classification of atrial fibrillation were completely different. Instead of the morphology of a single bipolar atrial electrogratin, we used the complete pattern of activation of the free wall of the right atrium as obtained by high resolution mapping (244 points). In type r fibrillation the right atrium was activated by broad wavefronts propagating rapidly and without 
significant conduction delay. The activation during type u was more complex showing a higher degree of delayed conduction and intra-atrial conduction block. During type ni, actiwation was highly complex, with the right atrium being activated by three or more wavelets that frequently reentered either themselves (leading circle reentry) or each other (random reentry).

In both studies, the average atrial rate increased from type I to type m. Wells et al, ${ }^{22}$ measured an average atrial cycle length of 179 milliseconds in type 1 and 151 milliseconds in type rr fibrillation, whereas in our patient population the different types of fibrillation showed an average median fibrillation interval of 174, 150 and 136 milliseconds with an average range of 54,94 and 104 milliseconds, respectively $\left(\mathrm{P}_{5.95}\right)$. Because of the totally chaotic nature of the bipolar recordings in the study of Wells et al., the atrial rate during type in fibrillation could not be accurately determined. This points to an important limitation in the use of widely spaced bipolar electrodes for the characterization of atrial fibrillation. As shown by our activation maps, during type in fibrillation atrial acrivation is highly dissociated. Consequently, two distant electrodes will frequently record from different activation waves rather than record a differential signal of a single depolarization wave. Under these circumstances, a widely spaced bipolar electrogram should be regarded as a dual unipolar electrogram representing two irregular clissociated rhythms that can not be used to determine the fibrillation rate. Thus, for the recording of endocartial or epicardial electrograms of atrial fibrillation either unipolar or closely spaced bipolar electrodes $(<1 \mathrm{~mm})$ should be used.

\section{Mapping of Atrial Fibrillation}

Mapping of atrial fibrillation has been performed in a number of studies. ${ }^{5+5 i n}$ ar In some of these studies, atrial fibrillation was sustained either by cholinergic stimulation, ${ }^{5,45,54}$ hypokalemia, ${ }^{56}$ or atrial enlargement due to mitral valve incompetence. These studies indicate that atrial fibrillation is based on reentry. The various cases disclose a wide spectrum of activation patterns, ranging from a single macro-reentrant circuit to numerous mutually reentering wavelets.

In our studies of cholinergic fibrillation in canine hearts ${ }^{54,55}$ and in those by Wang et al, ${ }^{54}$ total mapping of both atria exhibited complex activation patterns resulting from the interference of an average of about six individual wavelets. Obviously, "fine" atrial fibrillation as present during vagal or cholinergic stimulation is associated with the most complex activation patterns, classified in this study as type in fibrillation. 
In humans, only one other mapping study of atrial fibrilation has been reported. ${ }^{5 *}$ This study described findings in 13 patients with wip syndrome (age, $1.5-36$ years) who were operated on for division of an accessory atrioventricular connection. In all patients, atriall fibrillation was induced by burst atrial pacing. In six patients, atrial activation patterns were found that were suggestive of reentry in the vight atrium. In some of them, functional conduction block appeared to be associated with an underlying structure, such as the crista terminalis. The cycle length of right atrial reentrant circuits ranged between 180 and 210 milliseconds. "The left atrium was activated non-uniformly by wavefronts emerging either from the right atrial reentrant circuit or the accessory atrioventricular pathway. Documentation of complete left atrial reentry was rare. In the remaining seven patients, reentry could not be condusively demonstrated, but the repetitive sequence of activation of the atria was compatible with a large reentrant atrial pathway party through the inter-atriall septum. ${ }^{58}$

When we compare our results with the fibrillation maps shown in the paper of Cox et al., we conclude that in all of their cases, the pattern of activation of the right atrial free wall was consistent with type I fibrillation. This implicates that during type I fibrillation, the broad uniform wavefronts propagating across the free wall of the right atrium may be part of a large reentrant circuit around one of the natural anatomic obstacles present in the atria, with dimensions considerably larger than the diameter of our mapping electrode $(3.6 \mathrm{~cm})$. In contrast, during type m fibrillation, we frequently observed shifting leading circle reentry in the right atrium with a diameter of only one to two $\mathrm{cm}$. The fact that in the study of Cox et al. ${ }^{58}$ no maps of type mibrillation were shown, might be due to the lower spatial resolution used in that study, in which a total of 156 electrodes was divided over the total epicardial surface of both atria. The resulting relatively large interelectrode distance of about one cm makes it diffoult to reconstruct the more complex activation patterns of type un fibrillation, and the underlying shifting micro-reentrant circuits might be easily missed.

\section{Types I, II, and III Fibrillation: Do They Represent Different Mechanisms?}

Atrial fibrllation is a clinical diagnosis based on the absenct of regular $\mathrm{p}$ - or to waves and the presence of irregular $R-$ intervals. However, as emphasized by the present study, it is likely that from a pathophysiological point of view, atrial fibrillation is not a homogeneous arthythmia. The electrocardiographic characteristics of atrial fibrillation can be caused by several different electrophysiological mechanistms: (I) 
a single fixed source of rapid impulses that are not followed in a regular $1: 1$ fashion by all parts of the atrial myocardium (fibrillatory conduction), (2) a single shifting source of rapid impulses (wandering circuit), (3) two or more asymchronous sources of rapid impulses (para-systolic fibrillation), and (4) multiple propagating wavelets rentering each other or/and themselves (multiple wavelets).

The recent mapping studies, both in humans and dogs, have failed to demonstrate a significant role of antomatic foci in the perpetuation of atrial fibrillation. With the exception of its initiation, atrial fibrillation is shown to be entirely based on abnormaluties in intra-atrial conduction. Our present study shows that type in fibrillation in humans is based on multiple reentering wavelets. On the other hand, type 1 fibrillation might be more consistent with a single macro-reentrant circuit giving rise to irregular activation of other parts of the atria. From a pathophysiological point of view, type 1 fibrillation actually might also be regarded as a case of atrial flutter, (possibly type Ir flutter) with such a high rate that it can not be followed in a 1:1 fashion by all parts of the atria. If this were true, type $\mathrm{I}$ atrial fibrillation might be a good candidate for termination by rapid pacing. ${ }^{7}$

\section{Study Limitations}

In the present study, atrial fibrillation was mapped in a group of young patients (average age, 32 years) with the wp -syndrome. In these patients, the atria are apparently normal and showed a normal spread of activation during sinus rhythm. and rapid atrial pacing. Thus, the patient population of the present study is not representatiwe for the group of patients with clinical paroxysmal or chronic atrial fibrillation. The incidence of atrial fibrillation largely increases with age and is reported to be about $4 \%$ in the general population older than 60 years of age ${ }^{*}$ Also, the conduction properties of the atrium have been shown to change with age because of the development of intra-atrial fibrous septa, thus providing numerous anatomic substrates for conduction block and reentry. Alhowgh it has recently been demonstrated that the presence of an accessory atrioventricular connection does not affect the vulnerability of the atria to fibrillate in response to rapid atrial pacing, it is unknown to what extent the existence of an accessory pathway affects the characteristics of the fibrillation process. In addition, the mechanisms underlying spontaneous atrial fibrillation in the elderly or in cardiac patients may be different from electrically induced self-terminating atrial fibrillation as studied in our group of patients. 
Another important limitation of this study is that in most patients recordings were made of only one area (the right atrial free wall) during one episode of atrial fibrillation and that only one 12-5econdsample was analyzed. Obvously, this makes it hazardous to extrapolate our findings both to other parts of the atria and to other episodes of atrial fibrillation. In eight of 25 patients, we were able to analyze the patterns of excitation of both the right and left at rial free wall during the same episode of atrial fibrillation. In these patients, no najor differences were found between the right and left atria in type of fibrillation, local fibrillation intervals, and variation in fibrillation intervals. However, because the group of patients is rather small, general conclusions can not be drawn about spatial differences in activation patterns during atrial fibrillation. Because we analyzed only a single episode of atrial fibrillation in each patient, we can not address the question whether temporal variations among the three Iypes of fibrillation may occur.

\section{Clinical Implications}

At present, the clinical relevance of our observations is not clear. Is the pacing induced arrhythmia the same as spontaneously occurring atrial fibrillation, on is it more comparable to polymorphic ventricular tachycardia, which can be induced in the normal ventricle during aggressive ventricular pacing? In this respect, it is important that in 15 of the 25 patients the induced arrhythmia lasted more than two minutes, suggesting a sustained and stable nature of the arthythmia. In the seven patients who had clinical documentation of atrial fibrillation, no preference for one of the three types of induced atrial fibrillation was found, suggesting that during spontaneous atrial fibrillation, different numbers and dimensions of intraatrial circuits may be present. The question remains how often during atrial fibrillation fluctuations in number and dimension of intra-atrial circuits occur in the same patient. This might be relevant to ablative procedures to prevent those circuits from occurring. It is also of importance when pacing techniques are considered for termination and prevention of atrial fibrillation. Observations during spontaneously occurring episodes of atrial fibrillation are required to answer these questions. 



\section{Chapter 3 | Configuration of Unipolar}

Atrial Electrograms during Electrically Induced Atrial Fibrillation in Humans | by Karen T.S. Konings MD'; Joep L.R.M. Smeets MD, PhD*; Olaf C. Penn MD, PhD; Hein J.J. Wellens MD, PhD; Maurits A. Allessie MD, PhDt.

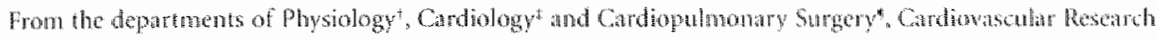
Instiute Manstrinht. 
On the basis of recording single bipolar atrial electrograms, in 1978 Wells et al. ${ }^{22}$ distinguished four different types of atrial fibrillation (Af) occurring within the first week after cardiac surgery. Type I was characterized by discrete complexes separated by an iso-electric basehne free of perturbations. Type In also showed discrete atrial complexes, but now the baseline between the major deflections, to a varying degree, was disturbed by smaller deflections. In type nir no discrete complexes could be distinguished and the bipolar electrograms were described as being "totally chaotic in nature." Type IV actually was a mixture of the other types, in which episodes of type in were alternating with electrogram morphologies as seen during type 1 or 11 fibrillation.

Using a high density mapping electrode with a diameter of $3.6 \mathrm{~cm}$ containing 244 unipolar electrodes, we mapped the actiwation of the free wall of the right and left atria during electrically induced atrial fibrillation in 25 wp patients undergoing cardiac surgery." On the basis of the degree of complexity of the activation maps, three types of atrial fibrillation were distinguished. In type 1 , the atria were activated by broad activation waves propagating uniformly with a still relatively high conduction velocity of about $60 \mathrm{~cm} / \mathrm{s}$. During type li fibrillation, arcs of functional intra-atrial conduction block were present and the mapping area was activated by two different fibrillation waves. In type rm the atria were activated in a highly complex manner by multiple slowly conducting wavelets $(38 \mathrm{~cm} / \mathrm{s})$ separated by multiple lines of functional conduction block, which continuously shifted in size and location. In this most complex type of a f, long episodes of continuous electrical activity were recorded in the free wall of the right and left atria. Like in the study of Wells et al. ${ }^{72}$ also temporal variations in the type of activation were conmonly observed, but instead of classifying this as a separate type of $A \mathrm{~B}$, we considered this as a general feature of atrial fibrillation.

Numerous studies have investigated the effects of factors like slow conduction, ${ }^{k 2}-88$

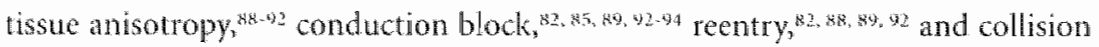
of activation waves, ${ }^{45}$ on the morphology of unipolar and bipolar electrograms. However, it is still unknown to what extent these various factors contribute to the temporal and spatial variation in electrogram morphology as observed during atrial fibrillation. Obviously this seriously limits the interpretation of fibrillation electrograms. Since high-density mapping can only be used in a limited and selected number of cases, it would be useful if single fibrillation electrograms 
recorded during catheterization could be "translated" into certain types of conduction disturbances. This would not only help to distinguish different types of atrial fibrillation, but it might also enable to ceter structural or functional abnormalities in the atrial walls responsible for perpetuation of atrial fibrillation. The aim of the present study was to analyze the various morphologies of unipolar atrial fibrillation electrograms and to deternine their relationship wh the underlying spatial patterns of activation.

METHODS

\section{Patient Population}

The study group consisted of the same 25 patients with a w $\mathrm{Pw}$-syndrome as described previously. ${ }^{8}$ Sixteen patients were malle and nine were female. Ages ranged from 12. to 57 years (average $32 \pm 11$ ). All patients underwent a pre-operative electrophysiologic study, during which the accessory pathway(s) were located. None of the patients had any other cardiac abnormalities than those related to the wPw-syndrome (as certified by electrocardiography, chest $x$-ray, echocardiography and coronary angiography). In seven patients atrial fibrillation was documented previously by EcG on Holter recordings. Antiarrhythmic medication was discontinued at least four days prior to surgery. None of the patients was taking amiodarone. All patients were operated before 1992 when radiofrequency ablation became the primary treatment of the wPw-syndrome. Informed consent was obtained prior to surgery.

\section{Experimental Protocol}

After anesthesia with fentanyl $\left(50-100 \mu \mathrm{g} \mathrm{kg}^{-1}\right)$, alfentanil ( $2 \mu \mathrm{g} \mathrm{kg}^{-8} \mathrm{~min}^{-1}$ ). and propofol ( $2 \mathrm{mg} \mathrm{kg}^{-1}$ hour-1) a median sternotomy was made and the heart was exposed. A hand-held spoon-shaped mapping electrode (diameter, $3.6 \mathrm{~cm} ; 244$ unipolar electrodes; interelectrode distance, $2.25 \mathrm{~mm}$ ) was used to accurately localize the accessory pathway(s) intra-operatively and to map the apicardial activation of the free wall of the right atria. Before the patients werc put on cardiopulmonary bypass, one or more episodes of atrial fibrillation were induced by rapid atrial pacing. In all patients the epicardial excitation pattern of the free wall of the right atrium was mapped both during sinus rhythm, rapid atrial pacing (interwal, 180 milliseconds) and a period of electrically induced $A{ }^{*}, A$ silver plate (diameter $2.5, \mathrm{~cm}$ ) in the thoracic cawity was used as indifferent 
electrode. Simultancously wh the 244 unipolar epicardial electrograms, a surface eco (leads,$I$, mand avr) and four bipolar reference electrograms were recorded from the right and left atria and from the ventricles. After amplification (gain, 500-1000), the 244 unipolar atrial electrograms were filtered (bandwidth, $1-500 \mathrm{~Hz}$ ), multiplexed (sampling rate, $1 \mathrm{kHz}$ ), $\mathrm{AD}$ converted (8 bits) and stored on videotape for subsequent analysis. ${ }^{25}$

\section{Data Analysis}

lin each patient one episode of atrial fibrillation was analyzed. From a period of stable atrial fibrillation a time frame of four seconds was selected and the 244 unipolar electrograms were transferred to a personal computet. The various morphologies of the unipolar atrial electrograms recorded during Af were classified into four categories: 1) single potentials, 2) short-double potentials, 3) long-double potentials, and 4) fragmented potentials. In Figure 3.1 an example of these types of electrograms is given. Single potentials are characterized by a single rapid negative deflection preceded by an $\mathbb{R}$-wave and smoothly returning to the baseline. Double potentials were defined as two negative deflections, the amplitude of the smallest being at least $25 \%$ of the amplitude of the largest. By using this criterion only double wavefronts within a distance of slightly more than about one space constant are included. Short-double potentials were defmed as double potentials with a time interval between the two deflections of less than 10 milliseconds. Long-double potentials were defined as electrograms showing two components separated by $10-50$ milliseconds. The upper limit of 50 milliseconds was based on the assumption that the atrial refractory period during $A$ and the interval between two successive fibrillation waves was at least 50 milliseconds. Fragmented potentials were defined as electrograms exhibiting more than two negative deflections within 50 milliseconds. All 244 electrograms recorded during four seconds of $\mathrm{Al}$ in 25 patients were displayed individually for inspection and each complex was classified according to the criteria given above. Electrograms that were difficult to judge, either due to superimposed high amplitude ventricular activity or poor tissue contact were discarded. The total data base of all unipolar ecterograns recorded during atral tibrillation comprised a total of more than 15 thousand individual complexes.

To compare the electrogram morphology with the spatial characteristics of activation, high density activation maps were reconstructed from the local activation times of all 244 recording sites. The software used for construction of 
Single Potentials

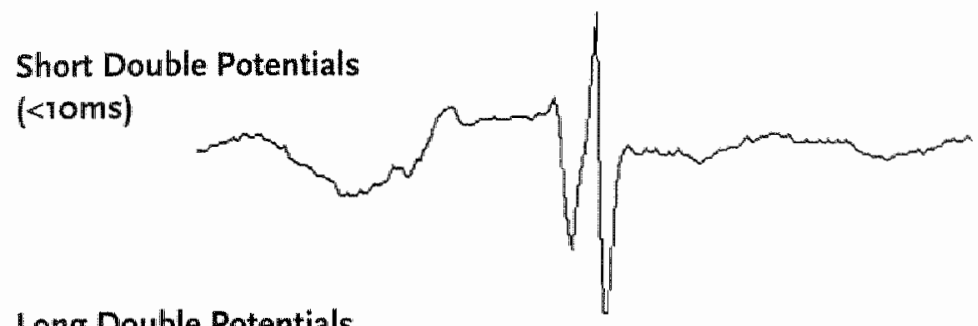

Long Double Potentials

(10-50 ms)

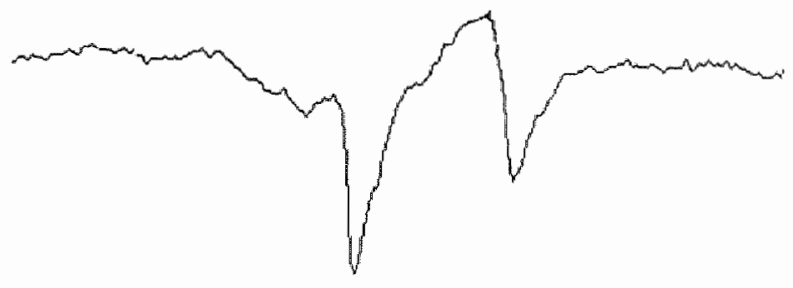

\section{Fragmented Potentials}

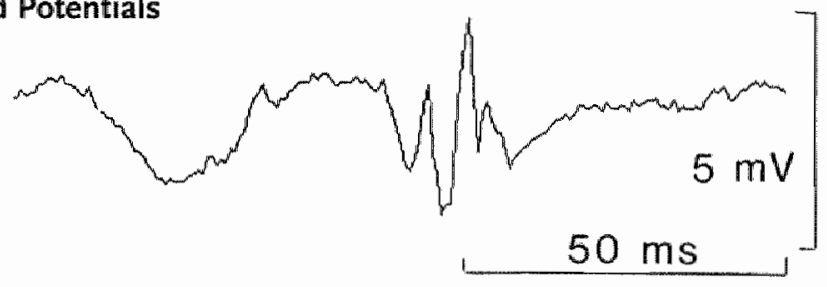

Classification of unipolar electrograms as recorded during atrial fibrillation. Single potentials are characterized by a single large negative deflection. Short-double potentials exhibit two negative deflections (the amplitude of the smallest being at least $25 \%$ of the largest) separated by less than 10 milliseconds. Long-double potentials are composed of two potentials separated by an interval of more than 10 milliseconds. Fragmented potentials show multiple negative deflections resulting in a prolonged duration of the activation complex. All unipolar electrograms were recorded by a spown-shaped mapping electrode (244 electrodes) positioned on the epicardium of the right atrial free wall, using a silver plate in the thoracic cavity as a common reference electrode. 
the activation maps included an automatic detection algorithm for the most negative intrinsic deflection, generation of color-coded activation maps, and interactive editing of local activation times..$^{75}$ Isochrones were drawn by hand at 10-milliseconds intervals. Inter-atrial conduction block was defined as a time difference of more than $30 \mathrm{~ms}$ between two neighboring electrodes (apparent local conduction velocity, $<7.5 \mathrm{~cm} / \mathrm{s}$ ) associated with a change in direction of propagation distal to the line of block (isochrones perpendicular to the line of block). Slow conduction was defined as a conduction time of more than 30 milliseconds associated with continuation of conduction in the same direction (isochrones parallel to the zone of slow conduction). ${ }^{88.95}$ A pivoting point was defined as the end of a line of functional block, where the impulse makes a $u-$ turn. If fibrillation waves propagated towards each other, the line of collision was defined by the electrodes that were activated later than each of their neighbors.

\section{Statistical Analysis}

Results are expressed as average $\pm S D$. Since the number of complexes varied in different patients, the proportion of the different categories of unipolar electrograms and their association with the local activation patterns are expressed as the percentage of the total number of complexes in each patient. Differences between groups were determined by Bonferroni's modification for small groups of the $t$-test. A P-value of less than .05 was considered statistically significant. The relationship between electrogram configuration and spatial activation characteristics was expressed by the sensitivity, specificity, and the negative and positive predictive values.

Resutirs

\section{Proportion of Different Types of Unipolar Atrial Electrograms}

In Figures 3.2 and 3.3 the proportion of the different types of electrograms is given during various atrial rhythms. During sinus rhythm and rapid pacing the far majority of electrograms showed single high amplitude rapid negative deflections $(93 \pm 3 \%$ and $92 \pm 3 \%$, respectively). During induced atrial fibrillation this high proportion of single potentials decreased to $77 \pm 12 \%(p<.05)$ (right upper panel Figure 3.2). In the different subgroups of Af (lower panels Figure 3.2) the percentage of single potentials decreased from $88 \pm 7 \%$ in type I fibrillation, to $76 \pm 6 \%$ in type 11, and to $6.4 \pm 7 \%$ in type $111(p<.05)$. The proportion of short- 


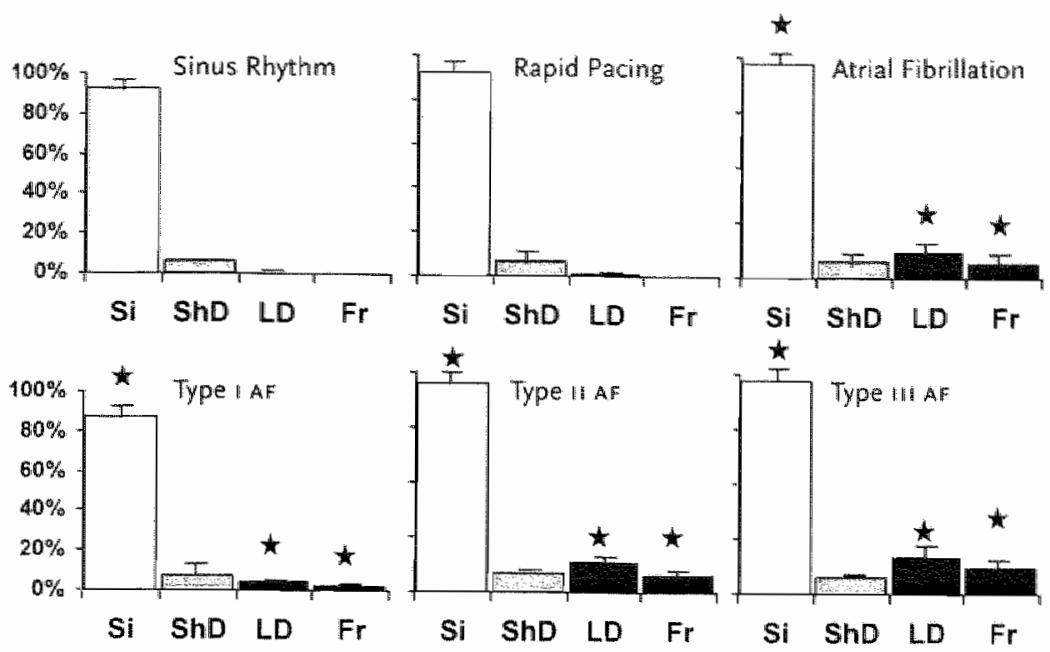

The distribution of single potentials (Si), short-double potentials (ShD), long-double potentials (LD), and fragmented electrograms (Fr) during various atrial rhythms in as WPWpatients with electrically induced AF. During atrial fibrillation the proportion of single potentials was significantly lower, and the proportion of long-double and fragmented potentials significantly higher, than during sinus thythm or rapid pacing (*, $P<.05)$. The percentage of short-double potentials was not different. From type I to type $111 \mathrm{AF}$, the proportion of long-double and fragmented electrograms progresisively increased (lower pamely $(*, P<.05)$.
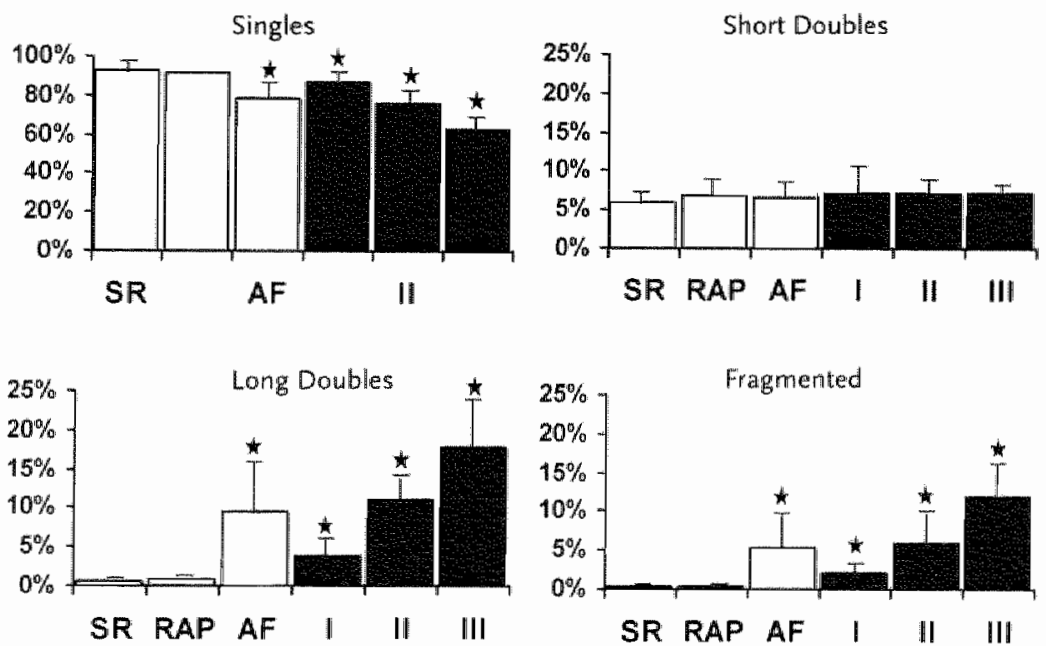

The proportion of each electrogram morphology during warious thythms, (sinus rhythm (sk), rapid atrial pacing (RAP), atrial fibrillation ( $A F)_{1}$ and type,$\|$ and $\| 11$ atrial fibrillation). AF was compared to SR and RAP (", $P<05$ ). The three subtypes of AF were compared to SR, AAP and the other two types of AF (", $P<05)$. See lext for description. 
double potentials during AF was the same as during sinus rhythm or rapid pacing, and averaged $7 \pm 3 \%$ (Figure 3.3 right upper panel). Whereas during type 1 fibrillation (ten patients) both long-double and fragmented complexes were relatively rare ( $4 \pm 2 \%$ and $2 \pm 2 \%$, respectively), in type in fibrillation (eight patients) these potentials occurred in $12 \pm 3 \%$ and $6 \pm 3 \%$. During type ar fibrillation (seven patients) long-double potentials comprised $18 \pm 7 \%$ of all complexes whereas $10 \pm 4 \%$ of the electrograms were fragmented (lower panels Figure 3.3). During sinus rhythm or rapid atrial pacing, no differences in electrogram morphology were found between patients with type I, II or III fibrillation, nor between the groups of patients with $(n=7)$ or without $(n=18)$ spontaneous episodes of af (Table 3.1).

As shown in Figure 3.4, the percentage of long-double and fragmented potentials was related to the atrial fibrillation rate. In this figure the relative proportion of longdouble and fragmented complexes in each of the 25 patients, is plotted against their median AF interval. Although there was a large interindividual variation, on the average the percentage of long-double and fragmented potentials was higher at shorter fibrillation cycle lengths. The correlation coefficients of the calculated linear regression lines were 0.47 and 0.38 , respectively, with $p$-values of .019 and.05, indicating a weak but significant correlation.

\section{Configuration of Unipolar Electrograms and Local Patterns of Activation}

To get more insight in the mechanisms behind the different morphologies of unipolar electrograms during atrial fibrillation, we correlated the shape of the electrograms with the local activation maps. In Figure 3.5 this relationship is illustrated for some specific patterns of activation like collision (A), conduction block ( $B$ ), pivot points (C), and slow conduction (D). All maps were recorded from the same area during a single episode of AF. Examples of the electrograms recorded during these events are given around the maps. In panel $\mathrm{A}$, four wavelets entering the mapping area from different directions (arrows), collided along the dashed line. The open squares on the map indicate electrodes where short-double potentials were recorded. As can be seen, almost all short-double potentials were located along either side of the line of collision. In panel $\mathbf{B}$, an example of functional conduction block is given. The wave entering the upper part of the mapping electrode at $t=0$ was blocked at $t=20$ milliseconds as indicated by the thick line. The other wave, entering the mapping area from the lower right at $t=10$ milliseconds, propagated rapidly from right to left along the line of block created 


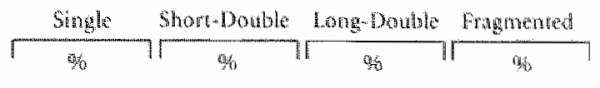

\begin{tabular}{|c|c|c|c|c|c|}
\hline \multirow[t]{3}{*}{ Sinus Rhythm } & SPONTANEOUS A $(n=18)$ & $93.2 \pm 3.0$ & $6.2 \pm 2.3$ & $0.5 \pm 0.3$ & $0.2 \pm 0.2$ \\
\hline & No DoGumented af $(\mathrm{n}=7)$ & $93.2+2.5$ & $6.0 \pm 2.3$ & $0.5 \pm 0.4$ & $0.2 \pm 0.2$ \\
\hline & $\mathbb{P}_{\text {in: }}$ & 0.88 & 0.85 & 0.97 & 0.98 \\
\hline \multirow[t]{3}{*}{ Rapid Aurial Pokcing } & SPONTAMEOUS AF $(N=18)$ & $92.3 \pm 3.7$ & $6.7 \pm 3.3$ & $0.7 \pm 0.5$ & $0.3 \pm 0.1$ \\
\hline & No DoCUAEMTED af $(n=7)$ & $92.0 \pm 2.3$ & $6.9 \pm 2.3$ & $0.8 \pm 0.6$ & $0.5 \pm 0.2$ \\
\hline & $\mathrm{P}=$ & 0.83 & 0.87 & 0.75 & 0.13 \\
\hline \multirow[t]{3}{*}{ Atrial Fibrillation } & SPONZANEOUS AR $\{n=18\}$ & $78.9 \pm 9.9$ & $5.9 \pm 1.8$ & $8.9 \pm 6.5$ & $5.1+4.7$ \\
\hline & No DOCUMENTED AE $(n=7)$ & $76.8 \pm 1.2 .6$ & $71 \pm 3.5$ & $10.3 \pm=7.7$ & $5.8 \pm 4.5$ \\
\hline & $P=$ & 0.69 & 0.41 & 0.67 & 0.73 \\
\hline
\end{tabular}

Af indicates atrial fibrillation.
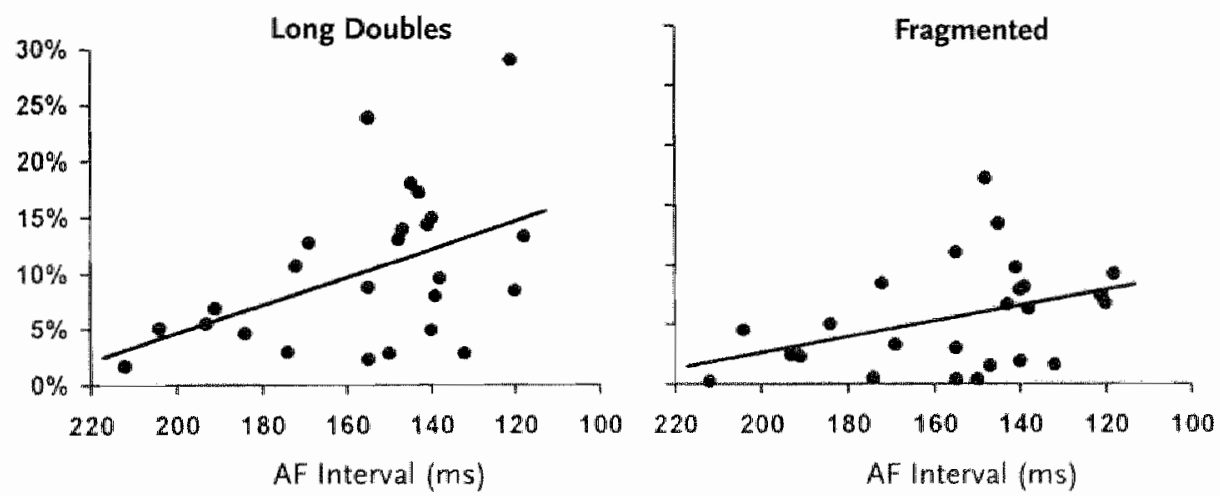

The relationship between the median atrial cycle length (AF interval) and the proportion of long-double and ragmented potentials during ellectrically induced atrial fibrillation. In the Figure 3.4 group of 25 patients the median fibrillation interwal waried between 212 and 118 milliseconds. Each data point represents the percentage of long-double (left panel) and fragmented potentials (right panel) during four seconds of AF in each individual patient. At higher rates of atrial fibrillation (shorter median Af intervals), the percentage of multi-component electrograms was higher than during slower fibrillation. The drawn lines are the calculated linear regression lines with correlation coefficients of 0.47 and 0.38 and p-values of 019 and 05 , respectively. 
by the first wave. The filled squares on the map indicate electrodes from which long-double potentials were recorded. Most long-double complexes, were recorded allong the line of conduction block. In panel $c$, an activation map is shown in which three wavelets turn around the ends of arcs of functional conduction block (curved arrows). The asterisks at these pivot points indicate electrodes from which fragmented potentials were recorded. Finally, in panel $D_{\text {, }}$ an example is given of slow conduction. In this example a single wave entered from the left $(t=0)$ and propagated to the right in 130 milliseconds. This long conduction time was caused by a zone of slow conduction in the middle of the mapping area where a crowding of 7 isochrones with a total conduction delay of $60 \mathrm{t}=20$ milliseconds can be seen. The asterisks in this band of crowded isochrones indicate the electrode sites where long fragmented complexes were recorded.

In Figure 3.6, the phenomena shown in Figure 3.5 are given in greater detail. All unipolar electrograms (interelectrode distance, $2.25 \mathrm{~mm}$ ) recorded in an area of about $1 \mathrm{~cm}^{2}$ are displayed as signal maps, with the specific electrogram configurations being highlighted. To illustrate the functional nature of the various specific electrogram configurations, and to show the temporal variation in configuration due to beat-to-beat changes in the underlying spatial pattern of propagation, at each panel a one-second tracing of one of the electrograms is shown. The site of recording of the specific electrogram configuration is indicated by a dot. In panel $\mathrm{A}$, the electrograms with short-double potentials can be accurately compared with the position of the line of collision (dashed line). The short-double potentials were recorded about one electrode distance $(2.25 \mathrm{~mm})$ from either side of the line of collision. Apparently one component is caused by actual passage of one of the colliding wavefronts under the electrode, while the other component must be an electrotonic potential. Note that the theoretically expected monophasic positive waveform at the site of collision"s was not seen. Actually the electrograms recorded from the exact site of collision showed single biphasic potentials.

From the signal map in panel $\beta$ it can be seen that the two components of the longdouble potentials coincide with the two waves at either side of the line of functional conduction block, the interval between the two components being proportional to the time difference in activation at either side. Since in this example the lower wave propagated from right to left, the interval between the two components along the arc of block also progressively increased from right to 


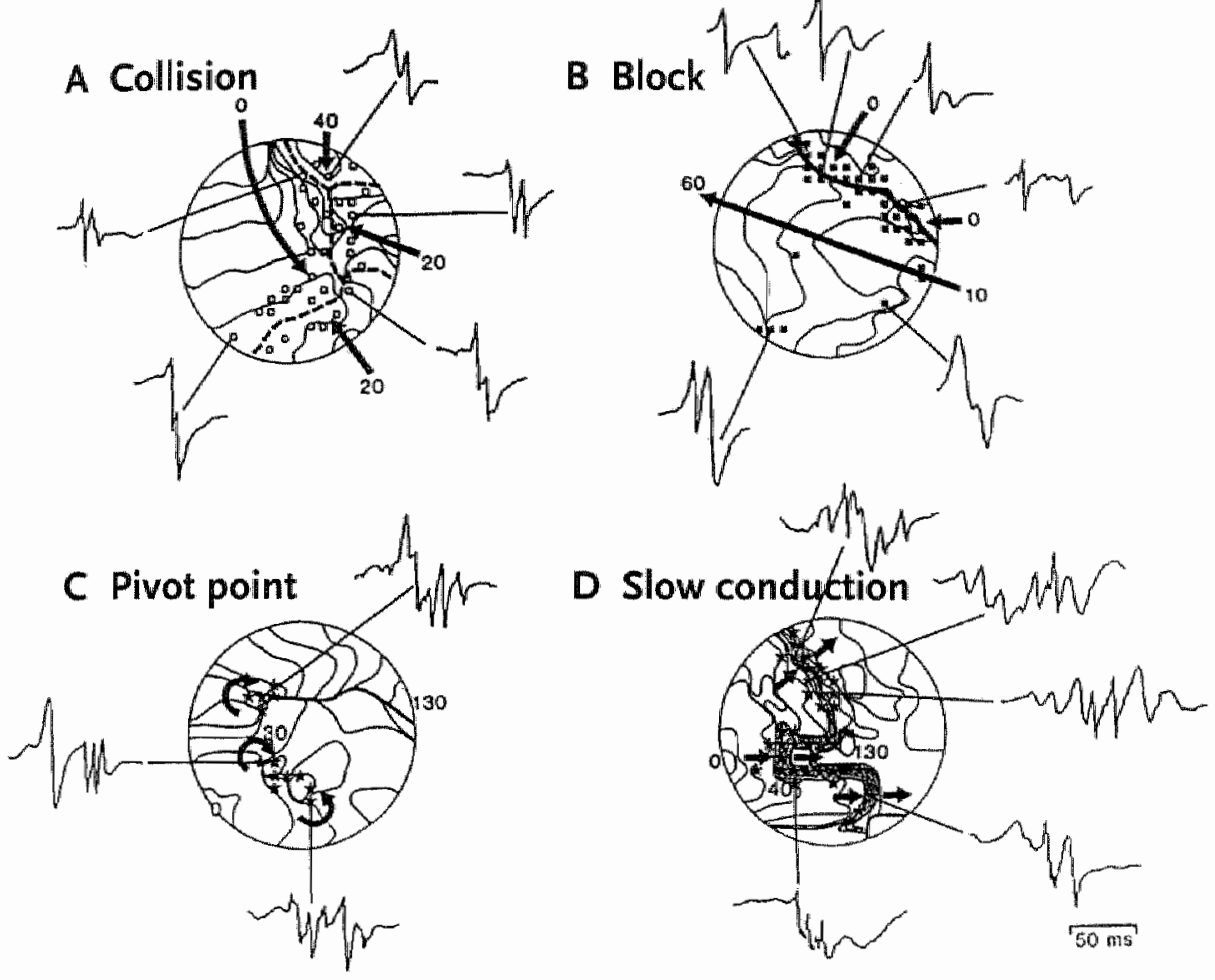

Comparison of the electrogram morphology and the underlying pattern of activation as Figure 3.5 recorded during Af. In panel $A_{*}$ four wawefronts (arrows?) invade the mapping area from different directions and collide along the dashed line. Hong either side of this line of collision short-double potentials were recorded ( $\square$ ). In panel s, long-double electrograms ( to be recorded at a line of functional conduction block (thick line). Fragmented electrograms (*) were recorded both at pivot points (panel c) and from areas with slow conduction (crowding of isochrones) (panel b). Isochrones were drawn at 10-milliseconds interval. Arrows indicate the direction of activation. Numbers indicate activation times sn milliseconds. 
A Short Doubles
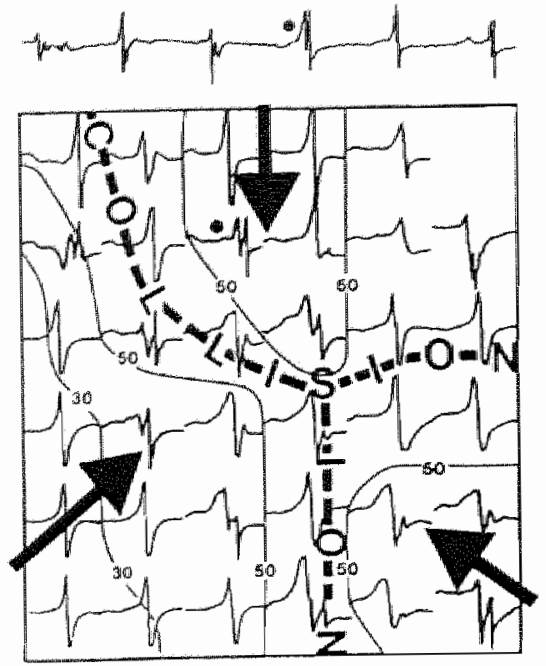

C Fragmented

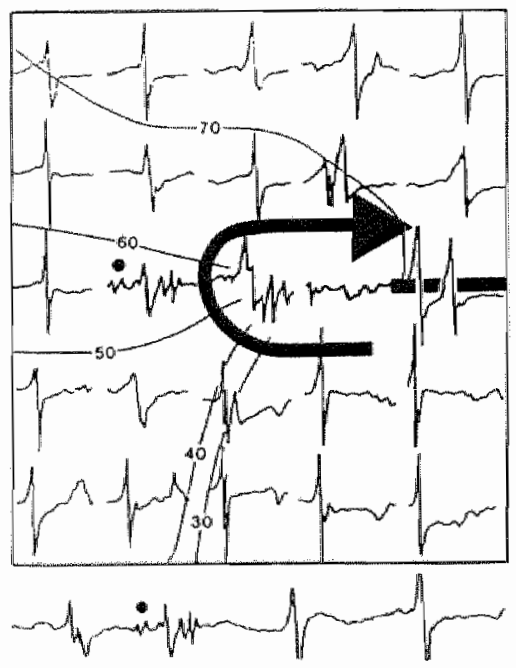

B Long Doubles
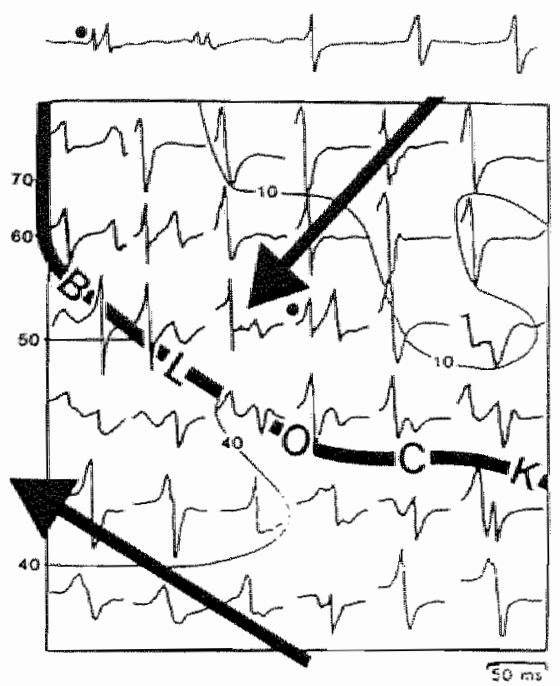

D Fragmented

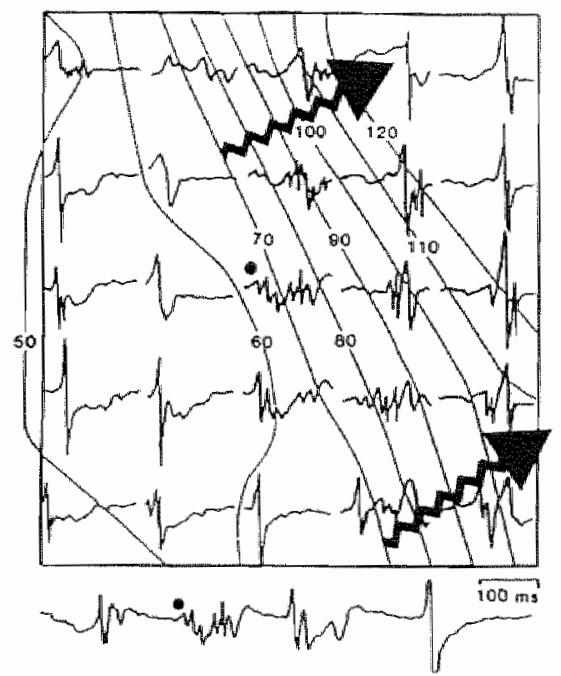

Figure 3.6

Signal maps from part of the isachrone maps given in figure 3.5 . The unipolar electrograms were recorded at relative distances of $2.25 \mathrm{~mm}$. isochrones are drawn by hand with the activation times given ir milliseconds. The dashed line in panel A indicates a line of collision. Intra-atrial conduction block is indicated by the thick line in panel a. The curved arrow in panel crepresemts the clockwise u-turn of a wawefront around the end of a line of functional conduction block. In panel a the crowding of isochrones between $t=60$ and t=120 illustrates an area of slow conduction of less than $6 \mathrm{~cm} / 5$. The electrograms exhibiting a specific configuration associated with these spatial patterns of activation are highlighted. To illustrate the functional nature of the specific electrogram configurations and their beat to beat changes in morphology, of each panel one electrogram is selected to show a number of successive beats (tracing of 880 milliseconds duration). The site of recording of these electrograms is indicated on the signal map by a black dot. See text for description. 
left (compare the double potentials at the lower right and the upper left part of the signal map).

Panel $c$ shows the signal map from a pivot point. At the site where the impulse makes

a sharp U-turn, the double component electrogram recorded at the line of block (thick line) is replaced by fragmented electrograms consisting of multiple discrete deflections. The various components of the fragmented electrograms at the pivot point coincide with the large single potentials recorded at some distance around the pivot point.

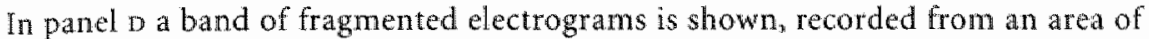
slow conduction $(6 \mathrm{~cm} / \mathrm{s}$ between isochrones 60 to 120$)$. The fragmentation of the electrograms was completely functional in nature; during sinus rhythm and rapid atrial pacing, this area demonstrated perfectly normal electrograms. Also during atrial fibrillation most of the time the complexes recorded from this area consisted of normal single potentials.

\section{Electrogram Morphology during Different Types of Atrial Fibrillation}

As described previously, the activation of the right atrium during atrial fibrillation shows large inter-individual differences. ${ }^{81}$ In Figure 3.7, examples are given of each of the three types of atrial fibriliation as distinguished on the basis of an increasing degree of complexity of atrial activation. Of each patient a single unipolar electrogram during four seconds of atrial fibrillation is shown, together with four activation maps at one-second intervals. In the diagrams below the maps the corresponding spatial distribution of the different electrogram morphologies are plotted. During type I fibrillation, the broad fibrillation waves predominantly produced single potential electrograms (indicated by dots). Scattered throughout the atrial wall and varying from beat-to-beat, also some short-double complexes were recorded (open squares). When two waves collided, short-double potentials were clustered at either side of the line of collision (map 3). However, as can be seen in map 1, some short-double potentials were also found during apparently uniform conduction (see later). The occasional occurrence of short arcs of conduction block (map 2) was associated with the recording of long-double potentials (filled squares). Fragmented electrograms (asterisks) were seen only rarely during type I fibrillation and mostiy as isolated spots at varying sites. During type in fibrillation (middle panel), more lines of functional conduction block were present and concomitantly the proportion of long-double potentials was higher (filled squares). Also more fragmented 
Type I

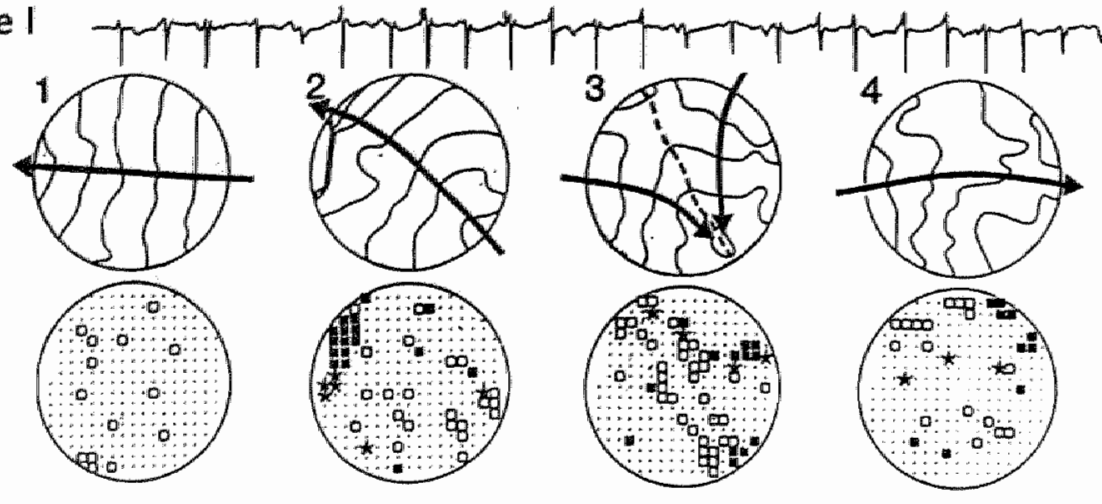

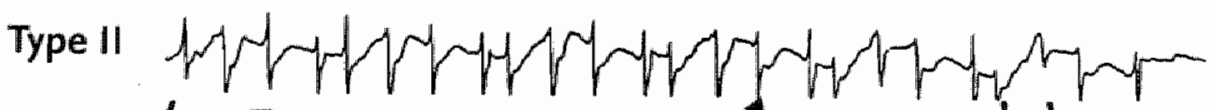
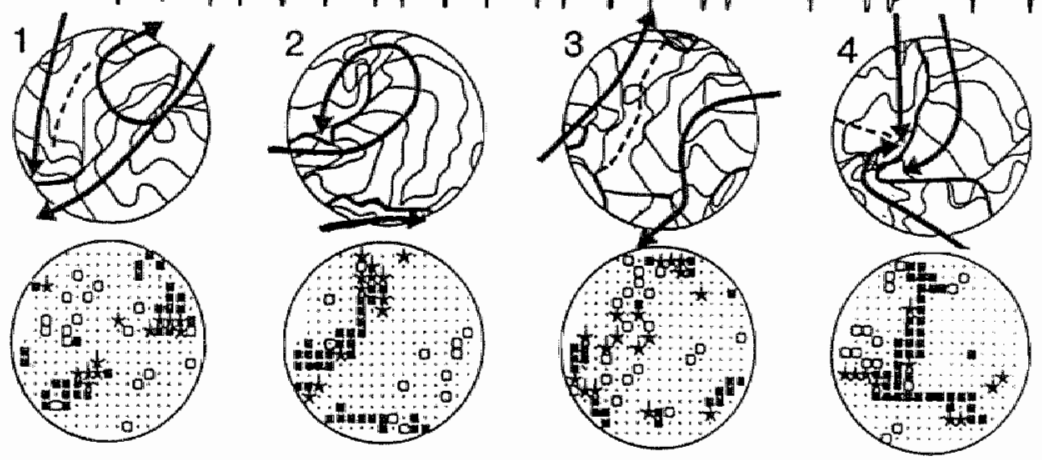

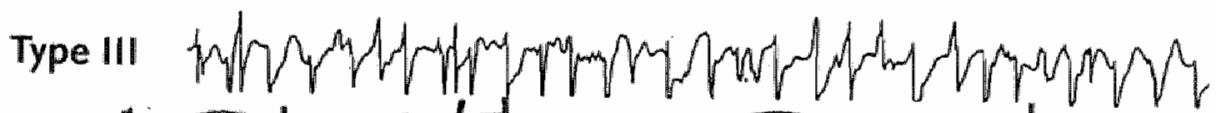
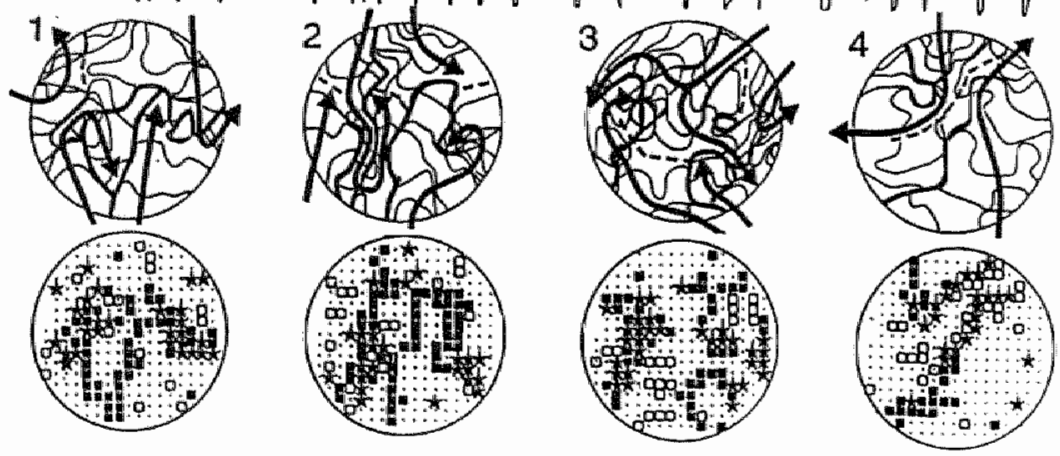
$\ll$ An example of the spatial distribution of the different electrogram morphologies during type I, type ill, and type 11 fibrillation. In each panel four seconds of af is shown. A right atrial unipolar electrogram is displayed together with four activation maps made at ane-second intervals (10- millisecond isochrones. Thick lines indicate block, dashed lines collision, alrows the direction of propagation). The diagrams below each map gives the spatial distribution of the electrogram configurations (- single potentials; $\square$ shortdouble potentials; longutouble potentials; * fragmented electrograms). During type i AF (upper panel) broad activation waves propagated rapidly in different directions across the free wall of the right atrium and the majority of the electrograms showed single potentials. Some short-double potentials (O) were found scattered throughout the myocardium (maps 1,2 and 4) or clustered along a line of collision (map 3). Only occasionally long-double potentials (were recorded at small arcs of conduction block (map 2). Fragmented electrograms were extremely rare. During type 11 fibrillation (middle pamel) a considerably larger number of long-double and fragmented potentials were recorded. However, no preferential areas for these multi-component electrograms were seen, and the beat to beat changing location of intra-atrial conduction block and slow conduction was distributed randomly. During type 41 fibrillation (lower panel), about one third of the electrograms had multiple components. This was due to the high degree of fragmentation of the fibrillation waves and the associated high incidence of slow conduction. conduction block, and piwoting of wavefronts. Also during type Ill fibrillation, no preferential sites for intra-atrial conduction block or sllow conduction were found in these patients.

electrograms (asterisks) were recorded, since during type u A slow conduction and pivoting fibrillation waves were more common. During type un fibrillation (lower panel), the free wall of the right atrium was activated by multiple fibrillation wavelets, which continuously collided, blocked, and pivoted around the ends of lines of block. Also areas of slow conduction were frequently seen. probably due to insufficient recovery of excitability during the short local fibrillation intervals. This complex pattern of activation resulted in a high number of long-double and fragmented potentials (together $28 \pm 6 \%$ of all complexes). Since the sites of block, pivot points, and slow conduction continuously changed, also the sites where long double and fragmented potentials were recorded changed from beat-to-beat. The spatial variation in activation patterns during atrial fibrillation thus, is expressed as temporal variations in unipolar electrogram characteristics recorded from a fixed site.

\section{Predictive Value of Electrogram Configuration}

In Figure 3.8 , for all 25 patients the different types of unipolar electrograms recorded during atrial fibrillation are correlated with the underlying local activation patterns. The data base consisted of a total of 517 fibrillation maps comptising 
more than 115 thousand complexes. Of these electrograms $79 \% 1 \%$ were recorded at sites showing uniform fast conduction, $3 \pm 2 \%$ during collision, $15 \pm 8 \%$ during conduction block, $2 \pm 2 \%$ at areas of slow conduction and $2 \pm 1 \%$ at pivot points. During broad fast conducting fibrillation waves, the greater majority of the recorded electrograms showed a single biphasic configuration $(94 \pm 4 \%)$. However, even during uniform conduction, $5 \pm 2 \%$ of the electrograms exhibited double potentials (see below). During collisior, conduction block, slow conduction or pivoting, the proportion of single potentials was only $22 \pm 13 \%, 14 \pm 7 \%, 33 \pm 27 \%$, and $17 \pm 12 \%$, respectively. Collision of wavefronts was characterized by shortdouble potentials $(65 \pm 5 \%)$. A lower proportion of short-double potentials $(15 \pm 6 \%)$ was found at lines of conduction block when the time difference in activation at either side of the line of block was relatively small (at the ends of the line of block). At arcs of intra-atrial conduction block long-double potentials prevailed (71 $18 \%$ ). Both during slow conduction and pivoting of fibrillation waves, fragmented potentials were most frequently recorded $(58 \pm 21 \%$ and $80 \pm 10 \%$, respectively). Of all recorded fragmented potentials, about half $(52 \pm 11 \%)$ was involved in a pivot point.

In Table 3.2 the sensitivity (se), specificity ( $\mathrm{sp}$ ) and positive and negative predictive values (PPV and NPV) of the electrogram morphology for the underlying pattern of activation are given. In the first columns of the table the relative proportion of the electrogram configurations associated with each pattern of activation is listed. As expected, single potentials were highly sensitive $(0.95)$ and specific $(0.84)$ to fast conduction, with a positive predictive value of 0.96 . Short-double potentials were most sensitive to collision $(0.69)$. The far majority $(98 \pm 2 \%)$ of the longdouble potentials recorded during collision of fibrillation waves (13 $\pm 9 \%$ ) had a time difference of less than 20 milliseconds between the two components.

Long-double potentials were highly sensitive to local conduction block $(0.75)$ with a positive predictive value of 0.84 . Fragmented electrograms were highly specific. both for slow conduction $(0.98)$ and pivot points $(0.99)$. The senstivity of fragmented electrograms was higher for pivot points than for slow conduction $(0.85$ versus 0.61$)$. From the high negative predictive value of 0.99 and 1.00 one. can conclude that in the absence of a fragmented electrogram the chance of slow conduction or turning of wavefronts is practically zero.

In Table 3.3 the positive predictive values of the different fibrillation electrogram morphologies for the various patterns of activation are summarized. Whereas single potentials, long-double potentials, and fragmented potentials were clearly 


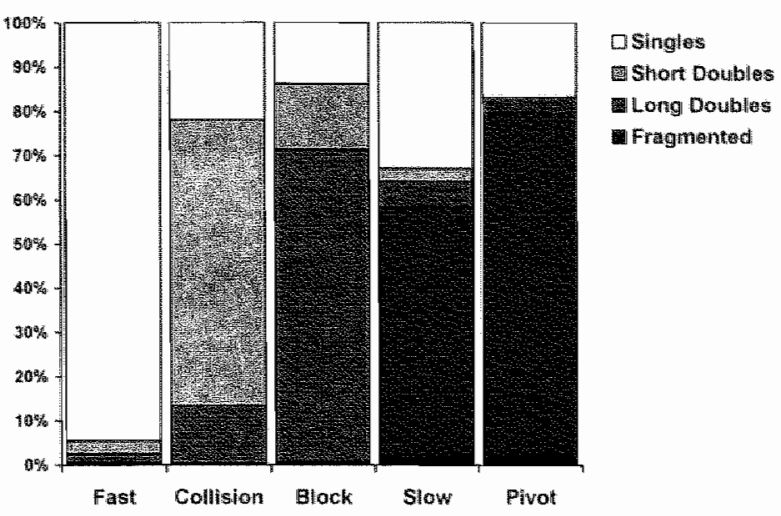

The association of different morphologies of unipolar electrograms recorded during spacific patterns of actiwation. The total data base consisted of more than 115 thousand electrograms. and 517 fibrillation maps, recorded in 25 patients. During uniform fast conduction, the electrograms almost exclusively had a single potential morphology $(94 \pm 4 \%)$. During collision, a large percentage of electrograms consisted of shott-double potentials (65 $\pm 15 \%)$. Longdouble potentials prevailed during conduction block $(71 \pm 8 \%)$, whereas fragmented electrograms were characteristic either for slow conduction $(58 \pm 21 \%)$ or pivot points. $(80 \pm 10 \%)$.

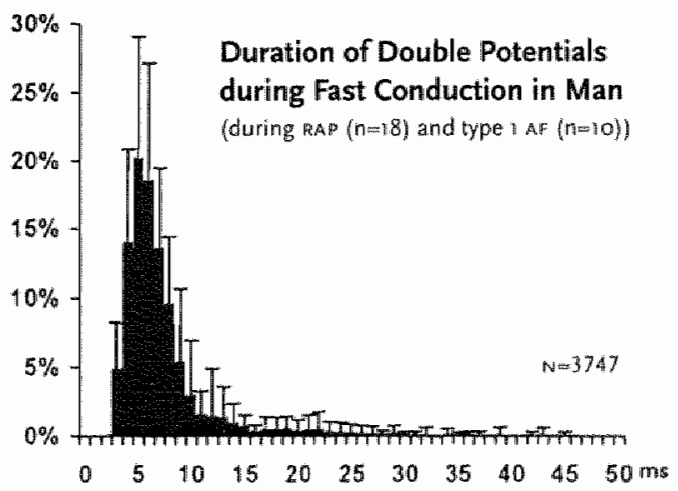

Histogram of the time interval between the two components of all double potentials recorded Figure 3.9 during fast uniform conduction during rapid atrial pacing (18 patients) and type I AF (10 patients). The median value of the time difference between the double potentials during uniform conduction was 6 milliseconds. The 90 and 95 percentile was 11 and 16 milliseconds respectivelly. 
indicative for a certain pattern of activation (fast conduction, conduction block, slow conduction/ pivot points), the presence of short-double potentials was about equally predictive for either fast conduction, collision, or conduction block (positive predictive values $0.36,0.33$ and 0.31 , respectively). As shown in Figure 3.9, double potential electrograms recorded during fast conduction exhibited only a small time difference between the two components with a median value of 7 milliseconds. The 90 and 95 percentile of the time differences between the double potentials during uniform conduction was 11 and 16 milliseconds respectively. This degree of spatio-temporal dissociation in intra-atrial conduction thus, obviously is a normal physiological property of human atria and short-double potentials should not be considered as a sign of abnormal atrial conduction.

\section{Identification of Areas with Intrinsic Conduction Disturbances}

In Figure 3.10 we have plotted the individual spatial distribution of all multicomponent electrograms (long-double plus fragmented potentials) recorded from the right atrial free wall during four seconds of atrial fibrillation. Shortdouble complexes were not taken into account. At each recording site, the relative percentage of long-double plus fragmented complexes is plotted in a gray scale (white $<0.25 \%$, black $>2.25 \%$ ). Patients with documented episodes of atrial fibrillation (indicated by asterisks) did not show an apparent different distribution than the others. During type a fibrillation the small number of nulticomponent electrograms tended to be clustered in certain parts of the mapping area. This is probably due to linking of the activation sequences ${ }^{97}$ and the relatively low number of multi-component electrograms recorded during type r fibrillation, rather than that it points to the presence of areas of impaired conduction. During type if and type in fibrillation the double and fragmented electrograms were more or less equally distributed over the mapping area and no preferential areas of conduction block, slow conduction or piwot points could be detected in the free wall of the right atrium. This was in agreement with the absence in these patients of any functional or structural intra-atrial conduction disturbances during sinus rhythm or rapid atrial pacing. ${ }^{\circ 1}$ Thus, in normal atrial myocardium the proportion of double and fragmented potentials during electrically induced AF was randomly distributed in space. 
Se indicates sensitivity: $S P_{2}$ Specificity: $P P_{0}$, positive predictive value: N $P V_{1}$ negatiwe predictive value.

\begin{tabular}{|c|c|c|c|c|c|c|}
\hline Fast Conduction. & SINGLES $(N=89790)$ & $94 \pm 4$ & 0.95 & 0.84 & 0.96 & 0.84 \\
\hline \multirow[t]{3}{*}{$(79 \pm 118 x)$} & SHORT-DOUBLES $(\mathrm{N}=7889)$ & $3^{\frac{5}{2} 2}$ & 0.03 & 0.82 & 0.36 & 0.19 \\
\hline & LoNG-Dovales (N= 1833) & $2 \pm 2$ & 0.02 & 0.48 & 0.12 & 0.12 \\
\hline & FRAGMENTED $(n=6496)$ & $0.4 \pm 0.5$ & 0.00 & 0.86 & 0.09 & 0.19 \\
\hline Collision & SingLeS & $22+13$ & 0.19 & 0.20 & 0.011 & 0.89 \\
\hline \multirow[t]{3}{*}{$(3+20 \%)$} & SHORTWDOUBLES & $65 \pm 15$ & 0.69 & 0.96 & 0.33 & 0.99 \\
\hline & LONG-DOUBHES & $13 \pm 9$ & 0.11 & 0.87 & 0.03 & 0.97 \\
\hline & FRAGMENTED & $0.3 \pm 0.8$ & 0.01 & 0.97 & 0.01 & 0.97 \\
\hline \multirow{4}{*}{$\begin{array}{l}\text { Conduction Block } \\
(15 \pm 84 \%)\end{array}$} & SIMGLeS & $14 \pm 7$ & 0.13 & 0.10 & 0,02 & 0.42 \\
\hline & \$HORT-DOURles & $15 \pm 6$ & 0.12 & 0.95 & 0.31 & 0.86 \\
\hline & LowG-Dovales & $71 \pm 8$ & 0.75 & 0.99 & 0.84 & 0.95 \\
\hline & FraGAENTED & $0.5 \pm 0.7$ & 0.01 & 0.96 & 0.03 & 0.85 \\
\hline \multirow{4}{*}{$\begin{array}{l}\text { Slow Gonduction } \\
(2+2 \%)\end{array}$} & SINGLES & $33 \pm 27$ & 0.32 & 0.21 & 0.01 & 0.93 \\
\hline & SHORT-DOUBLES & $3 \pm 5$ & 0.02 & 0.94 & 0.00 & 0.98 \\
\hline & LONG-DOUBLES & $6 \pm 9$ & 0.05 & 0.87 & 0.00 & 0.98 \\
\hline & FRAGMENTED & $58 \pm 21$ & 0.61 & 0.98 & 0.40 & 0.99 \\
\hline \multirow{4}{*}{$\begin{array}{l}\text { Piwot points } \\
\text { (2t) }\end{array}$} & SINGLES & $17 \pm 12$ & 0.10 & 0.20 & 0.00 & 0.92 \\
\hline & Srort-Dounlas & $0 \pm 0$ & 0.00 & 0.94 & 0.00 & 0.98 \\
\hline & Lomca-Donalies & $4 \pm 5$ & 0.05 & 0.87 & 0.10 & 0.90 \\
\hline & FRAGMENTBD & $80 \pm 10$ & 0.85 & 0.99 & 0.47 & 1.00 \\
\hline
\end{tabular}

Positive Predictive Value of Fibrillation Electrogram Morphology for Associated

"Wable 3.3

Patterns of Conduction

\begin{tabular}{|c|c|c|c|c|}
\hline & $\begin{array}{c}\text { Fust } \\
\text { Conduction }\end{array}$ & Collision & $\begin{array}{l}\text { Condution } \\
\text { Moeth }\end{array}$ & $\begin{array}{l}\text { Show } \\
\text { Condution } \\
\text { / Phom } \\
\text { Moints }\end{array}$ \\
\hline SIMALE POTENTAM & 0.96 & 0.01 & 0.02 & 0.01 \\
\hline 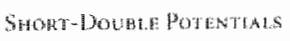 & 0.36 & 0.33 & 0.31 & $0.0 \%$ \\
\hline Long-DOUnLE POGENMALS & 0.12 & 0.03 & 0.84 & 0.01 \\
\hline WHAMENTED POURATILLS & 0.09 & 0.91 & 0.03 & 0.87 \\
\hline
\end{tabular}



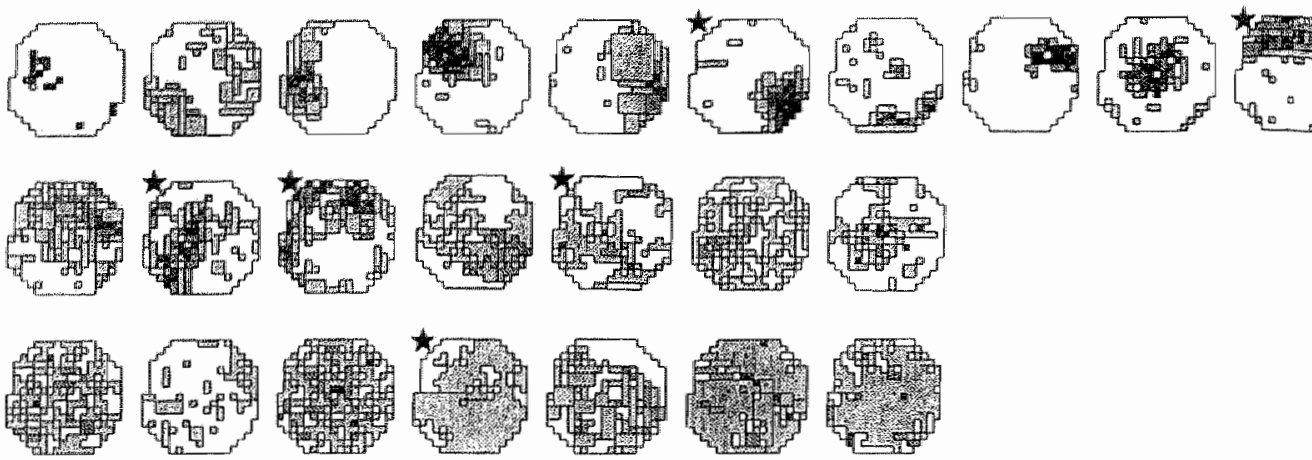

Figure 3.10

Spatial distribution of multi-component electrograms (long-double and fragmented potentials) during four seconds of electrically induced AF in the individual patients (types :-111). The mapping electrode with a diameter of $3.6 \mathrm{~cm}$ (244 electrodes) was positioned on the free wall of the right atrium. In the diagrams the percentage of multi-component electrograms recorded at each individual electrode is plotted in a gray scale. White areas indicate electrodes where less than $0.25 \%$ of the total number of multi-component potentials were recorded, light gray indicates a proportion between $0.25 \%$ and $2.25 \%$ and black is used to indicate sites where more than $2.25 \%$ of the multi-component electrograms were recorded. In patients with type I Af the total number of multi-component electrograms recorded during four seconds of fibrillation was rather small and the majority of the electrodes recorded less than $0.25 \%$ of the total number of multi-component electrograms (large white areas). Due to this small number, during type 1-Af, the spatial distribution of multh-component potentials could not be reliably determined. During type il and III fibrillation the multi-component electrograms were more or less equally distributed over the whole mapping area, indicating that in these patients no sites of preferential conduction block or slow conduction existed in the free wall of the right atrilum. Patients with spontaneous episodes of af are indicated by an asterisk. One of these patients (showing type 11 AF), was not included in this analysis because more than $20 \%$ of the atrial complexes were masked by high amplitude ventricular complexes. 


\section{Morphology of Unipolar Electrograms}

The waveform of extracellular electrograms recorded during propagation of a depolarization wave in atrial or ventricular myocardium has been shown to depend on many factors suchas the amplitude and upstroke velocity of the action potential, the direction of the wavefront relative to the fiber axis (anisotropy), discontinuities in electrical side-to-side coupling between (groups of) fibers, and asynchrony in activation of closely adjacent areas. ${ }^{79,87}$ The studies of Spach and coworkers showed that: "complex waveform shapes with multiple deflections ..... can be interpreted in terms of asynchronous excitation of small groups of fibers within distances of 50-100 $\mu \mathrm{m}^{\prime 3}$. Atrial fibrillation can be considered as an experiment by nature, in which a constant structure (the atrial myocardium) is subjected to a continuously changing pattern of excitation by multiple wavelets propagating at short coupling intervals and in different directions. Due to the accurrence of functional rate-dependent intra-atrial conduction block, frequently the activation of the atria becomes dissociated, either by turning of wavelets around a line of block, or by reentry by another wavelet traveling in a different direction (random reentry). In the present study the changes in configuration of unipolar fibrillation electrograms due to this continuously changing dissociation in conduction were studied.

\section{Short-Double Potentials}

During sinus rhythm and rapid atrial pacing $7-8 \%$ of the unipolar electrograms recorded from the free wall of the right atrium showed double potentials. The sires where these double potentials were recorded were scattered throughout the atrial wall and were not associated with any detectable slowing of the activation wave. Recently Schuesler et al.$^{60}$ have shown that during normal atrial activation of the canine right atrium, transmural differences in activation time up to 24 milliseconds occurred. Therefore, the short-double potentials recorded from the epicardium during fast propagation of broad uniform activation waves many reflect the normal physiologic dissociation in conduction between the transmural layers of the atrial wall. In the present study the double potentials recorded from the free wall of the human right atrium during uniform conduction, showed a median phase difference between the double components of 6 milliseconds. In 90 and $95 \%$ of the cases the time difference between double potentials recorded 
during normal conduction was less than 11 and 16 milliseconds respectiwely. 'Thus in our opinion shori-double potentials with a dissociation of not more than $10-15$ milliseconds should be considered as normal potentials, which express the physiological heterogeneity in architecture of the atrial wall.

During atrial fibrillation short-double potentials were also recorded at either side of lines of collision of tibrillation waves, reflecting a small phase difference between the wo waves, whereas at the exact sites of collision biphasic potentials were recorded. This was a somewhat surprising finding, since in other studies single monophasic positive potentials were found at sites of collision. 95 This discrepancy may be due to the different preparations used in these studies. Monophasic positive potentials were recorded in thin in vitro preparations, in which only the superficial cell layers remain intact. However, if recording from the epicandium of an intact perfused atrium, like in our study and that of Schuesler et al , ${ }^{60}$ also the intramural layers participate in the generation of epicardial potentials. This may explain why in the intact heart single biphasic potentials were recorded at sites of collision, flanked by dissociated short-double potentials. If at the moment of epicardial collision, in deeper layers of the atrial wall a depolarization wave is still propagating, this will generate a far field potential causing a negative deflection in the terminal part of the biphasic electrograms recorded from the epicardial line of collision.

\section{Long-Double Potentials}

Double potentials were first demonstrated at the central line of block of functionally determined circuits during mapping of rabbit and canine atriall reentry. ${ }^{53,88}$ Later studies showed that they can also be recorded from anatomical barriers both in the atria and the ventricles., ${ }^{93,94,99}$ Double spike electrograms can exist both during various tachyarthythmias and during sinus thythm or slow pacing ${ }^{\text {s.8 }}$, 114 there is general agreement now that they simply reflect asynchronous activation of tissue at either side of an area of block, regardless of its nature and independent of the presence of reentry. spatial distribution of double potentials during atrial fibrillation may be of value in two different ways: 3) The temporal distribution at a fxed recording site may give information about the proportion of conduction block and the degree of fragmentation of the fibrillation waves, without the need of high density mapping of the fibrillatory process. In this way, different types of fibrillation might be defined quantitatively and the effects of various antifibrillatory drugs could be 
evaluated. 2) Determination of the spatial distribution of double potentials might identify areas that are critical for perpetuation of atrial fibrillation. They may either point to anatomical abnormalities in the atrial wall (in case they occur at fixed sites) or to functional ellectrophysiological inhomogeneities. In this way double potentials might help to identify atrial areas that are critical for perpetuation of atrial fibrillation.

\section{Fragmented Electrograms}

Fractionated or fragmented electrograms have been recorded both in patients with atriall or ventricular arrhythmias and in animal models of tachyarrhythmias. ${ }^{5 .}$, at. $87-89 ; 94,98-102,106-117$ At present they are used to localize critical zones for atrial

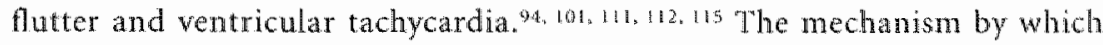
fragmented potentials are generated has been studied in detail by Wit et al. ${ }^{67.3 x^{3}, 18}$ and Spach et al. ${ }^{79}, 95$ These studies have shown that each component of a fractionated electrogram arises from action potentials in different muscte bundles, which are activated out of phase. In isochrone maps this results in crowding of isochrones. ${ }^{88}$ Lowal asynchronous activation can be due to various mechanisms; 1) spatial dispersion in refractory periods, 2) tissue anisotropy resulting in a zigzag course of the propagating depolarizing wavefront on a microscopic level due to a low number of electrical side-to-side connections, ${ }^{7 \%}, 11 \%$ 3) the presence of insulating collagenous septa between atrial muscle bundles. ${ }^{79}, 8$

\section{Clinical Implications}

Atrial fibrillation is the most common type of tachycardia with well known hemodynamic and thromboembolic implications. New information allowing possible cure from this arrhythmia is of obvious importance. Mapping studies, including our own, showed the rols of reentry in the genesis and perpetuation of atrial fibrillation. These observations led to an interest in approaches that prevent reentry mechanisms from occurring by creating anatomical batriers in the atriat wall. Examples of such approaches are the maze operation ${ }^{20}$ and the creation of lines in the atrium by catheter techniques. ${ }^{121}$ One of the challenges in dissecting the atrium to cure atrial fibrillation is to make the dissections in such a way that the contractility of the atrium is not seriously impaired. "That requires the smallest possible number of dissections and preferably in such a direction that synchronized atrial contraction is still possible. To do so the identification of possible critical areas for reentry during atrial fbrillation becomes mandatory. 
Athough the atria as a whole participate in the process of atrial fibrilation and each cell is rexcited at a high rate by the wandering wavelets, this does not necessarily mean that all parts of the atria contribute equally to the perpetuation of the fibrillatory process. It rather seems more likely that the pathophysiological changes associated with aging, or an underlying cardiac disease will affect different parts of the atria with a different time course and to a different degree. Although in our study population, patients who had experienced spontaneous episodes of atrial fibrillation showed no differences compared to patients in which af did not occur, in patients with chronic atrial fibrillation, parts of the atria showing a high proportion of multi-component potentials might point to structural or functional abnormalities of the myocardium which might play a role in the persistence of atrial fibrillation. As long as the structure of the atria does not show generalized pathological changes, selective ablation of the most seriously affected parts might be of value for the treatment of atrial fibrillation. In the light of what is presently known, arcs of intra-atrial conduction block, areas of slow conduction, and piwot points of turning wavelets, all seem to be important factors for perpetuation of fibrillation. Lines of conduction block separate the different wavelets and therefore are an index of the degree of fragmentation of activation. Areas of slow conduction shorten the wavelength of the wandering wavelets, thereby increasing the number of them that can coexist in the atria. Pivot points are crucial because, no matter how many wavelets are present, if they don't turn they will soon die out at the boundaries of the atria and fibrillation will terminate. Our present study shows that all these abnormal conduction patterns (slow conduction, functional conduction block, and pivot points) are "translated" into long-double and fragmented potentials. This offers the possibility to use these potentials recorded during Af as a guide to identify atrial areas eligible for radiofrequency ablation. In the future, selective ablation of such areas might offer an additional approach for the treatment of atrial fibrillation.

\section{Limitations of the Study}

The observations made during induced atrial fibrillation in wpw-patients with normal haemodyramics and normally sized atria can not be considered as characteristic for the arthythmia mechanisms in patients with paroxysmal or chronic atrial fibrillation. At present, we are not well informed whether preferential areas for reentry during Af exist in patients with spontaneous and/or persistent atrial fibrillation. If $\mathrm{s} 0$, we don't know where they are located and 
whether they are anatomically or functionally determined. It is not unlikely that those areas differ in location and properties in patients with "lone" atrial fibrillation and in patients with atrial fibrillation secondary to atrial enlargement as in mitral valve disease. Also, it is not known whether paroxysmal atrial fibrillation has the same arrhythmogenic mechanism as chronic atrial fibrillation. Since we only analyzed a single episode of atrial fibrillation in each patient and limited mapping was limited to the right atrial free wall, it remains unclear to what extent temporal or spatial variation in atrial fibrillation and electrogram configuration exists, and whether a single sample as analyzed in the present study is representative for the whole process of atrial fibrillation.

For all these reasons, one should be cautious to extrapolate the present data to clinical atrial fibrillation. Although it seems unlikely that the fundamental relationship between the various patterns of activation and the configuration of unipolar atrial electrograms would be basically different, it can not be excluded that in diseased atria different patterns of activation exist during atrial fibrillation and that atrial electrograms will show a much higher degree of fragmentation, either due to depressed action potentials or electrical uncoupling of fibers. Recently, "focal" patterns of activation have been reported in the free wall of the right atrium in some patients with chronic AF. ${ }^{122}$ Obviously, more mapping data in patients with atrial fibrillation of different etiologies and duration are needed, especially because such information may be a guide to design optimal dissecting interventions in the atria to cure atrial fibrillation. 



\section{Chapter 4 | Mapping of The Right}

Atrium during Acute and Chronic Atrial Fibrillation in The Goat | BY

Karen TS. Konings; Maurits C.E.F. Wijffels, MD,PhD Rick Dorland, BS; Frans Mast, PhD, Maurits A. Allessie, $M D, P h D$.

Department of Physiology Cardiowascular Research Institufe Malastricht. 
Atrial fibrillation is the most commonly encountered arrhythmia in clinical practice (6.3\% of emergency admissions ${ }^{2}$ ), being present in $0.4 \%$ to $0.9 \%$ of the population. ${ }^{24}$ The arrhythmia presents itself in various forms. In clinical likerature a classification in terms of the duration of attacks is used, that is, short lasting or acute atrial fibrillation (including paroxysmal, transient, recurrent, new, and recent-onset $A$ ) versus long lasting or chropic atrial fobrillation (including sustained, persistent or permanent af). In clinical practice it has been noted that paroxysmal atrial fibrillation often deteriorates into chronic fibrillation $(22 \%)$. ${ }^{125}$ 127 The transition is influenced by the underlying etiology (lone Af $18 \%,{ }^{126}$ rheumatic heart disease $66 \%$ ), ${ }^{129}$ and by the duration of paroxysms. 129 The pace of the transition from paroxysmal to chronic. Af is rarely investigated but seems to lie above $20 \%$ per decade. ${ }^{125-128}$ Apparently, also the incidence of paroxysms influences the speed of transition to chronic AF. Continuous induction of paroxysms of A in goats invariably induced chronic fibrillation within three weeks ${ }^{130}$ This is a serious problem since the risks of chronic af are higher than those of paroxysmal AF, 131.133 and in patients with chronic arrhythmia it is more difficult to restore the sinus rhythm. ${ }^{134}+1$ ast

The pattern of symptoms in many patients with paroxysmal AF suggests an etiologic role for the autonomic nervous system, whereas chronic AF is commonly secondary to structural cardiovascular dysfunction. 1435 The relation of chronic Af with structural changes is also reflected by the fact that patients with chronic Af are generally older than those with paroxysmal A F. Aging is associated with development of intra-atrial fibrous septa, ${ }^{79}$ providing an anatomic substrate for intra-atrialconduction block and reentry, and causing a greater stability of the arrhythmia.

Recently it has been shown, that artificial maintenance of Af in goats resulted in a progressive increase in the duration of A F, leading to sustained fibrilation $(>24$ hrs) after $1-3$ weeks. This deterioration was accompanied by an increase in af rate $(+26 \%)$ and inducibility of Af (from $24 \%$ to $76 \%$ ) ${ }^{\text {In }}$. In this study the wavelength of impulses during the short episodes of acute $\mathrm{Ar}$ was estimated to be 8 to $9 \mathrm{~cm}$, in contrast 105 to $6 \mathrm{~cm}$ during chronic Af Another recent study showed that the spatial coherence during chronic AF $(1.8 \mathrm{~cm})$ is less than during paroxysmal $A F$ $(2.8 \mathrm{~cm})$, indicating that the individual wavelets depolarize a smaller amount of tissue. These space constants were calculated to correspond to a wavelength of 15 
$\mathrm{cm}$ during acute AF and $12 \mathrm{~cm}$ during chronic fibrillation. In both studies the decreasing wavelength may contribute to the greater stability of the arrhythmit, by increasing the number of wavelets that can coexist in the atria. "We have previously used the number of wavelets in the right atrial free wall to classify atrial fibrillation into three types. However, we could not establish any relationship between the duration of the electrically induced episodes of atrial fibrillation and the degree of complexity of the activation pattern, due to the small number of patients studied. Nevertheless, it has been suggested that a higher degree of complexity of the atrial activity during atrial forillation may result in a greater stability of the arrhythmia, thus promoting its perpetuation.

The present study was designed to compare the activation patterns during acute and chronic af by high density mapping of atrial fibrillation in the goat model . ${ }^{130}$ "The results of this analysis support the hypothesis that an increase in the number of wavelets causes a higher stability of atrial fibrillation.

Methods

\section{Experimental protocol}

This study includes seven goats (weight $61.2 \pm 21.7 \mathrm{~kg}$ ) that were operated upon for implantation of multiple atrial electrodes and sacrificed $7.1 \pm 3.5$ months later. These goats took part in the chronic goat studies of Wiffels et al. $130,1+7-1850$ Animal handling was performed according to the guiding principles of the Dutch law and approved by the Animal Investigation Committee of Mastricht University. The

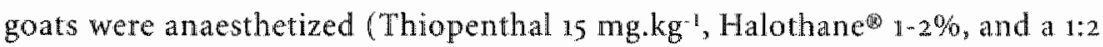
mixture of $\mathrm{O}_{2}$ and $\mathrm{N}_{2} \mathrm{O}$ ). A left intercostal thoracotomy was made, and the pericardium was opened to expose the heart. Threa silicon strips with platinnm electrodes were sutured to the lateral walls and the anterior transverse sinus between the atria and the aortic root. After closure of the chest, the electrode leads. were tunneled subcutaneously to the neck and exteniorized. Postoperatively, the animals received buprenorfine for $1-3$ days. Ampicilin (1000 mg) was given prophylactically both before and after surgery. "This procedure has been described in detail by Wijfels et al. ${ }^{130}$

Epicardial mapping of the free wall of the right atrium was performed first during acute atrial fibrilation induced before implantation of atrial electrodes, and repeated after chronic Af had persisted for $16.1 \pm 9.4$ weeks (range, 1 to 27 weeks). Mapping was performed with a hand-held spoon shaped electrode (diameter, 3.6 
cm) containing 244 unipolar electrodes (silver wires; diameter, $0.3 \mathrm{~mm}$ ) arranged

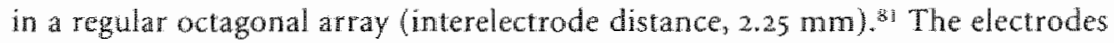
were connected to a multichannel recording unit (gain, 200-400; bandwidth, 1-500 Hz). ${ }^{75}$ All electrograms were stored on magnetic tape for subsequent analysis.

Acute af was induced by a one-second burst of biphasic stimuli (interval, 20 milliseconds; four times diastolic threshold). Chronic af was induced $2-3$ weeks after surgery by stimulation of the implanted epicardial electrodes by an automatic atrial fibrillator (a personal computer connected to a stimulator [Medtronic, SP3084]). When this automatic fibrillator detected simus rhythm, it promptly reinduced Af by burst pacing, thus maintaining AF for 24 hours a day, seven days a week. ${ }^{130}$ This resulted in a progressive increase in the duration of $\mathrm{Al}$ episodes, until ar became chronic within 13 to 63 days (average $29.4 \$ 16.5$ days).

\section{Data Analysis}

During off-line analysis, three time windows of four seconds of acute Af (taken at least four seconds after onset and tour seconds before termination of $A F$ ), one 12 * second sample of chronic AF, one four-second sample of sinus thythm and several frequencies of rapid pacing were selected from tape, and the signals were transferred to a personal computer for detailed analysis. Local activation times were marked and labeled as either single-, double- or fragmented potentials by the investigator according to the following criteria: Single potentials exhibit one or two sharp deflections in a maximum time window of 10 milliseconds. Double potentials exhibit two sharp deflections within 11-50 milliseconds, the amplitude of the smaller potential being at least $25 \%$ of the larger. Fragmented electrograms consist of three or more deflections. ${ }^{15}$ The total duration of the double potentials and fragmented electrograms was also registered.

Activation maps were reconstructed from the local activation times. Isochrones were drawn at 10-milliseconds intervals. Conduction block was defined as an apparent conduction velocity of less than $7.5 \mathrm{~cm} / \mathrm{s}$ (time difference between two adjacent electrodes, $>30$ milliseconds) combined with a clear change in the direction of conduction distal to the line of block. Slow conduction was defined as a local conduction velocity of less than $7.5 \mathrm{~cm} / \mathrm{s}$, with conduction continuing in the same direction distal to the area of slow conduction. Thus, the difference between blocked and slowed conduction was mainly the difference in direction of activation at either side of the arc of conduction disturbance. 
Each activation map was classified as type $r$, in or In activation according to the following criteria: Type activation was defined as a single wavelet conducting through the mapping area with only minor disturbances in conduction. During type rt activation, either a single wavelet was present combined with some considerable conduction block or slow conduction, or two wavelets were recorded simultaneously. Type rr was defmed as the presence of three or more wavelets under the mapping electrode. If more than $50 \%$ of the beats in an episode of atrial Abrillation was of type I or III, fibrillation was classified as type $\mathbb{I}$ or $1 \mathrm{H}$, respectively. Otherwise Af was classified as type ${ }^{\prime}$."

Fibrillation interval histograms were made from all 244 electrograms, and the median values were calculated. An indication of the Af refractory period was obtained by measurement of the shortest interval. The variation in local fibrillation intervals was expressed as the difference between the $5^{\text {th }}$ and $95^{\text {th }}$ percentile in the fibrillation histogram $\left(\mathrm{p}_{5-95}\right)$.

Conduction velocities (cv) were calculated in two different manners. During sinus rhythm, rapid atrial pacing and uniformly conducted beats during atrial fibrillation, the conduction velocity was calculated by taking three samples per beat, perpendicular to the isochrones. The distance was divided by the difference in activation times, and the average of the three was considered to be the conduction velocity. During sinus rhythm and several pacing rates in each goat, the average conduction velocity was calculated for a minimum of four beats. During Af, every uniformly conducted beat within 12 seconds was measured.

When conduction during atrial fibrillation was dissociated into multiple wavelets, the first method could not be applied, therefore another method was used which is illustrated in Figure 4.1. An example is given of a fragmented beat during chronic atrial fibrillation (goat 5). The local wectors of conduction were calculated by the difference in activation times of each triangle of electrodes (upper right pand). A histogram was then plotted from all local conduction welocities during the $12-$ second period that was analyzed (lower right panel). The advantage of this method is that the exact pathway of conduction needs not be determined. Nearly simultaneous activation of closely spaced electrodes sometimes resulted in apparent velocities of $>160 \mathrm{~cm} / \mathrm{s}$. This may happen when two wawes collide or when a wavelet appears somewhere in the middle of the mapping area (epicardial breakthrough). Since at these points there is no conduction from one electrode to the other, values $>160 \mathrm{~cm} / \mathrm{s}$ were discarded. The very slow apparent conduction welocities $(<7.5 \mathrm{~cm} / \mathrm{s}$ ) resulting from local conduction block were included, and 


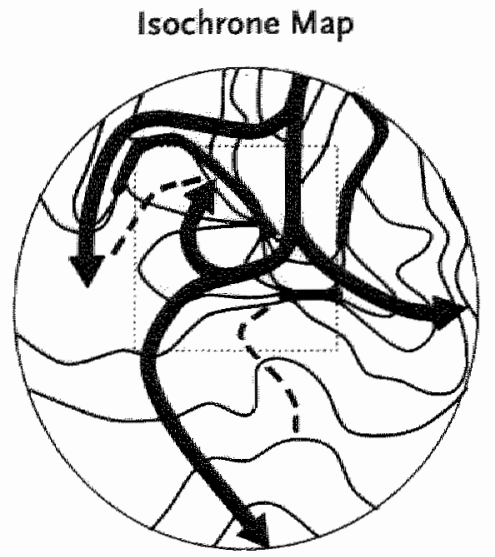

local Velocities $(\mathrm{cm} / \mathrm{s})$
Activation Times (ms)

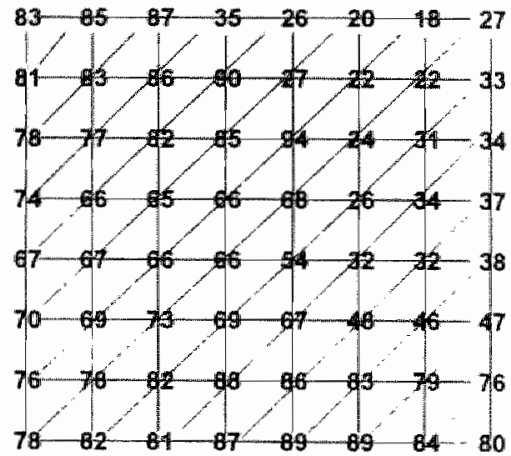

Histogram

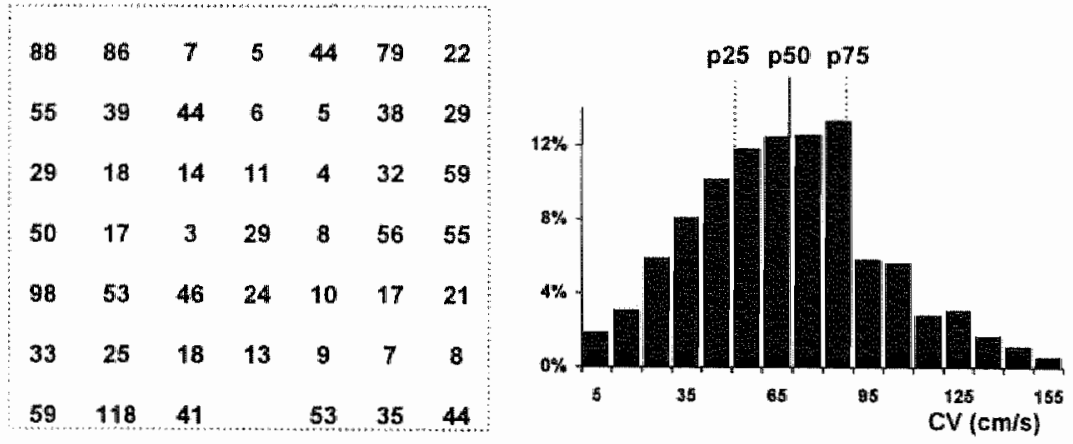

Figure 4.1

Method of calculation of conduction velocities during atrial fibrillation. Upper left: activation map of a fragmented beat during atrial fibrillation (goat 5). (sochrones are at 10-millisecond intervals (thin black lines). Several arcs of conduction block (thick black lines), a line of collision (dashed lines), and small areas of slow conduction (crowding of isochrones) can be seen. The general direction of conduction is indicated by arrows. The dotted square indicates the area of which activation times and local conduction velocities are showm. Upper right: activation times (in milliseconds), together with the triangles by which the conduction velocities were calculated. The distance between electrodes is $2.25 \mathrm{~mm}$. Lower left: the corresponding local conduction velocities (in $\mathrm{cm} / \mathrm{s}$ ). Only half of the values per four electrodes are plotted for clarity. Lower right: the histogram of all conduction velocities measured during 12 seconds of atrial fibrillation. The median value is indicated by a straight line $(67 \mathrm{~cm} / \mathrm{s})$, while the $P_{25}(44 \mathrm{~cm} / \mathrm{s})$ and $P_{35}(86 \mathrm{~cm} / \mathrm{s})$ are indicated by a dashed line. See text for further description. 
the median $\mathrm{cv}$ during the whole 12 -second sample of atrial fibrillation was calculated for each goat.

The average pathlength of fibrillation waves was estimated by multiplying the median fibrillation conduction velocity by the median fibrillation interval. "This pathlength represents a rough estimate of the average length of the route that the multiple wavelets travel before they reenter themselves or the tail of another wavelet.

\section{Statistical Analysis}

Data are presented as average $\pm S D$. Statistical analysis of the obtained results was performed using the paired Student's $t$-test. A corrected probability value of less than .05 was considered to be statistically significant.

ResuLTS

\section{Atrial Fibrillation Cycle Length}

In Figure 4.2 two representative unipolar right atrial electrograms during atrial fibrillation in the goat (7) are shown. Typically the electrograms of both acute AF (upper trace) and chronic fibrillation (lower trace) showed a rapid irregular rhythm with a continuous beat-to-beat variation in electrogram morphology and cycle length. The lower panel displays the summed interval histograms of all goats $(n=7)$. The average median interval during acute Af was $143 \pm 13$ milliseconds compared to $95 \pm 19$ milliseconds during chronic fibrillation $(-34 \%, p<.05)$. The width of the histograms remained the same $\left(P_{5-95}\right.$ during acute AF, 64 milliseconds versus 69 milliseconds during chronic $A F, P=43)$. The average shortest interval decreased from $66 \pm 19$ milliseconds during acute AF to $42 \pm 5$ milliseconds during chronic fibrillation $(-37 \%, p<05)$, indicating that the refractory period had shortened. The results of each individual goat are summarized in Table 4.1. The median intervals during acute $\mathrm{AF}$ were nearly the same as the mean interval (average difference, 2 milliseconds), indicating that the intervals were evenly distributed. After atrial fibrillation had been maintained for $4.2 \pm 2.4$ weeks, the difference between the median and the average fibrillation intervals increased slightly (to 8 milliseconds), indicating that the distribution of intervals had changed. Indeed the proportion of short intervals was larger during chronic AF. 
Acute AF

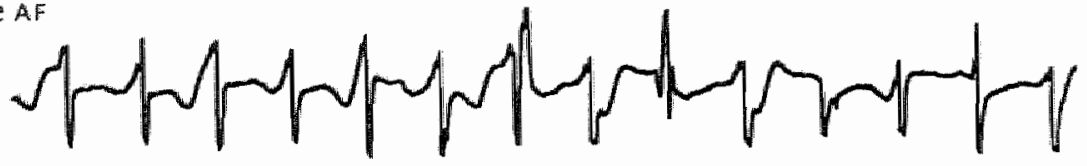

Chronic AF
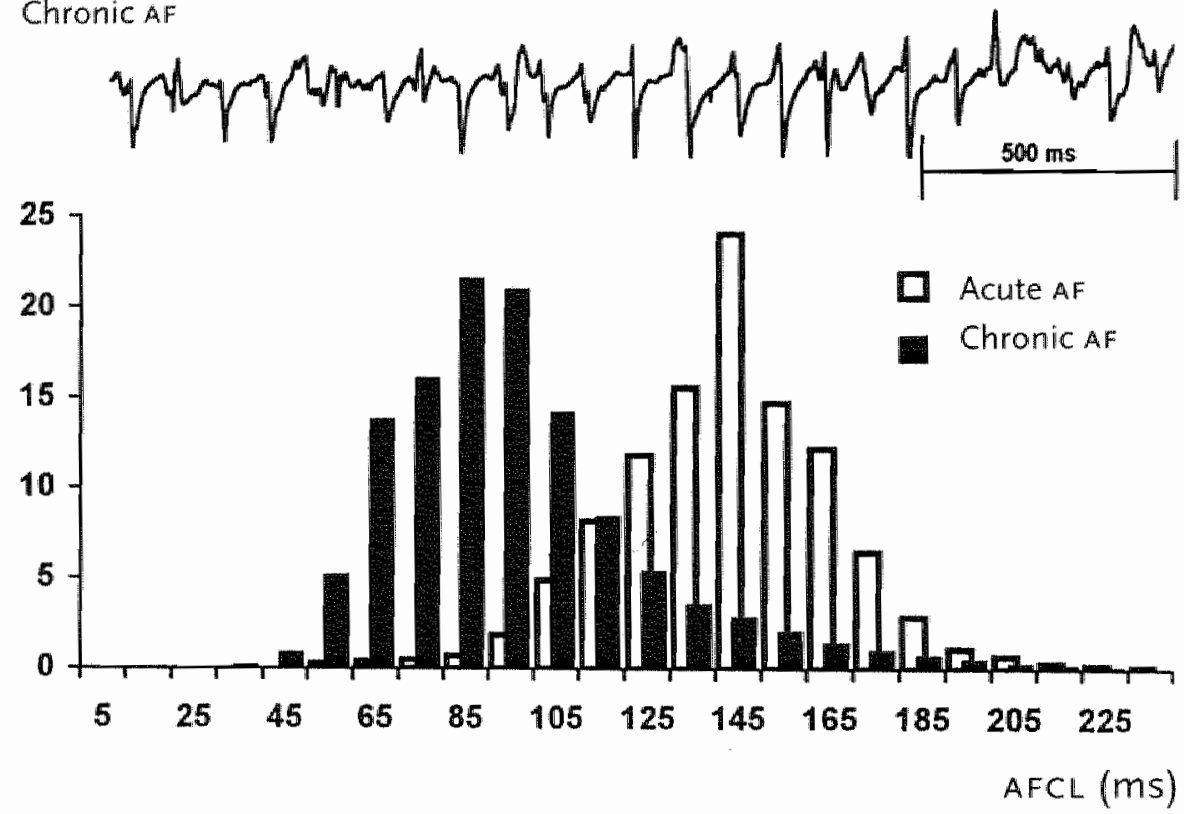

Figure 4.2

Right atrial unipolar electrograms of two seconds of acute AF (upper tracing) and chronic AF Nower tracingl in goat 7 . Below, the summed interval histograms of all goats are plotted. White bars represent the distribution during acute atrial fibrillation, black bars represent chronic AF. On the abscissa, the fibrillation interval (AFCL) is plotted. The median values are 143 milliseconds and 95 milliseconds respectively. On the ordinate, the number of measurements: $\times 1000$. 


\section{The Degree of Complexity of Activation}

Large differences between acute and chronic atrial fibrillation were observed in the degree of complexity of activation of the right atrial free wall. In Figures 4.3 an 4.4, two representative examples are given (goat 1). During acute atrial fibrillation (Figure 4.3) the activation pattern was still relatively uniform, and during the majority of beats the free wall of the right atrium was activated homogeneously by a single activation wave entering from above or from the right. During the whole period of 12 seconds, $74 \%$ of the activations were of type i Sometimes a second wavelet collided and/or fused with the other, or piwoted around the end of an arc of conduction block (beats $3,5,11$, and 12 ). In this goat, type IIr beats were not observed during acute AF. Because of the large beat-to-beat variation in conduction velocity, the total conduction time of the mapping area varied between 30 and 60 milliseconds.

After atrial fibrillation had become chronic, the activation patterns were much more complex (Figure 4.4). The median interval had shontened from 129 milliseconds to 1.07 milliseconds. Nearly every beat $(99 \%)$ now demonstrated three or more wavelets that were simultaneously present and collided and/or fused trequently (Type III). Several lines of conduction block were present, separating these wavelets. The lines of block constantly waried in location, indicating that they were functional in nature. Often the wavelets reentered tissue that had been activated before by another wavelet, and occasionally a wavelet reentered its own tail of refractoriness (dashed lines). Sometimes a wavelet arose de novo somewhere in the middle of the mapping area (asterisks), resulting in a focal spread of activation. The location of such focal points of entry varied. A measurement of the uniform conduction velocity could only be made during three beats (not shown), when one of the wavelets was broad and conducted along a straight line.

The distribution of the different types of activation during acute and chronic atrial fibrillation in the individual goats is given in Figure 4.5 and Table 4.1. In each goat the proportion of type 1 beats during chronic atrial fibrillation $(3 \pm 1 \%)$ was markedly lower than during acute $\mathrm{AP}(71 \pm 30 \%, \beta<.05)$. The amount of type 11 beats increased from $2 \pm 4 \%$ during acute $\mathrm{AP}$ to $70 \pm 38 \%$ during chronic fibrillation $(P<.05)$. The number of type in beats remained the same $(27 \pm 28 \%$ and $26 \pm 35 \%$ respectively, $p=61$ ). The overall type of fibrillation changed from Type a during acute af to Type in during chronic fibrillation. However, in the acute setting some goats exhibited Type II fibrillation ( 2 and 3 ) and in the chronic state some goals 


\section{Acute Atrial Fibrillation}
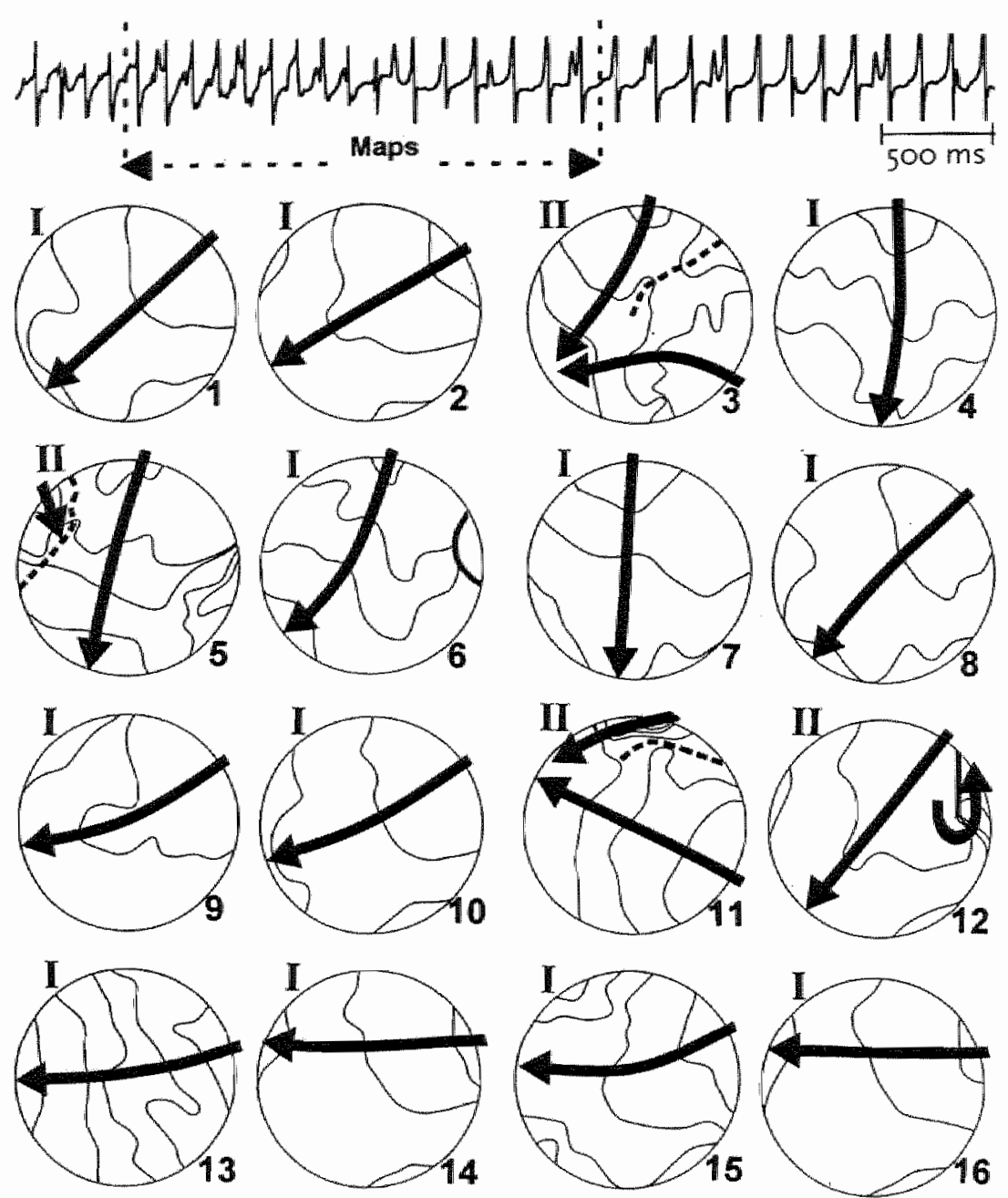

Figure 4.3

A right atrial unipolar electrogram (RA), and consecutive activation maps of the right atrial free wall during acute atrial fibrillation in a goat (1). The type of fibrillation and the sequence number of each beat is indicated at each map. Isochrones are drawn at 10 -milliseconds intervals. Thick lines represent arcs of conduction black, and dashed lines arcs of collision. Arrows indicate the direction of activation. In this case of acute atrial fibrillation, the average activation pattern was of type I fibrillation. See text for description. 


\section{Chronic Atrial Fibrillation}
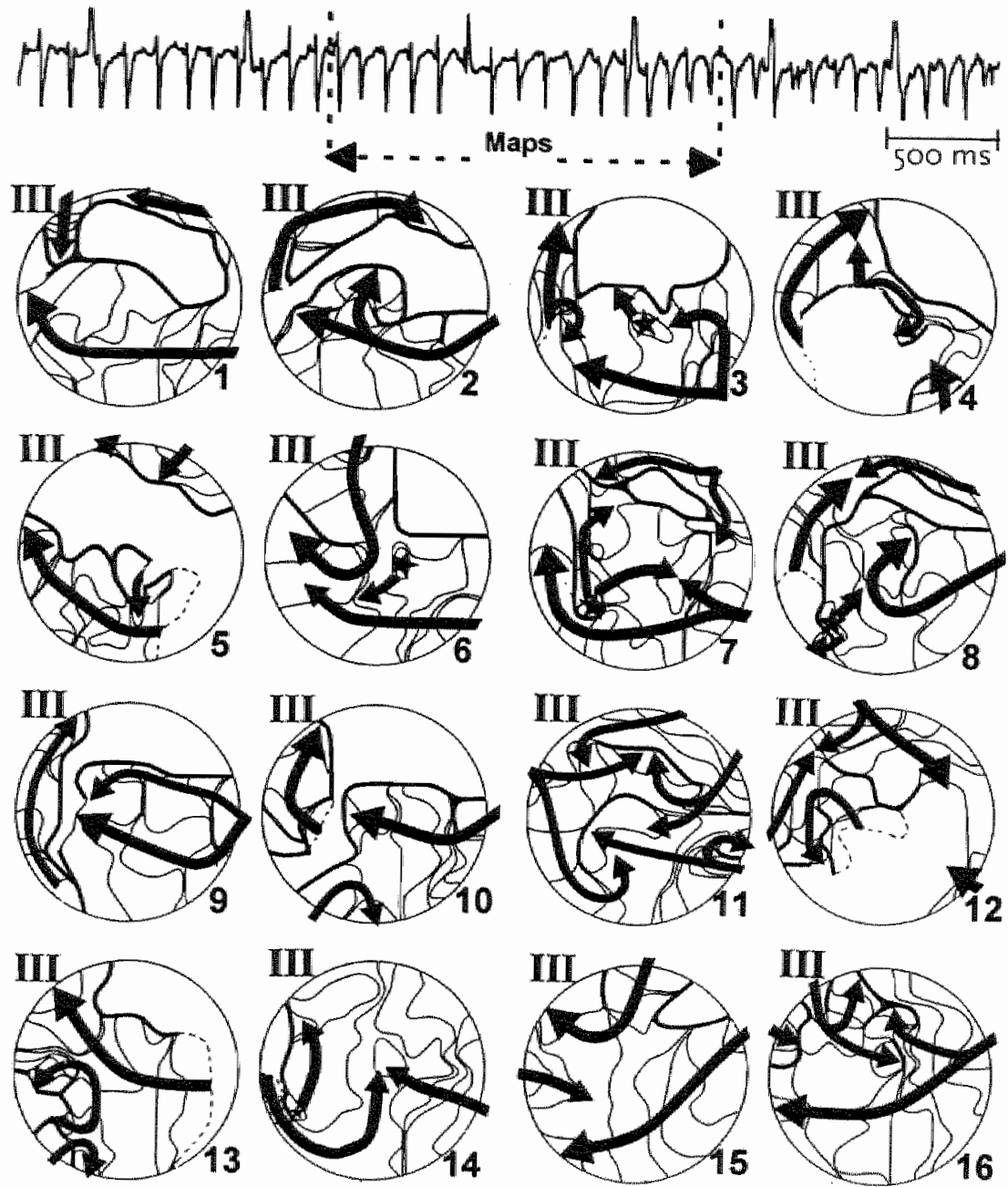

A right atrial unipolar electrogram (RA), and consecutive activation maps of the right atrial

Figure 4.4 free wall during chronic atrial fibrillation in the same goat as in Figure 3 . The maps are drawn in the same manner Crowding of isochrones depicts slow conduction. Colfision lines have been omitted for clarity reasons. Dashed isochrones indicate that a wavelet continues there from the previous map. Stars are plotted at sites of early activation in the middle of the map. Now the average activation pattern was of typelll. See text for description. 


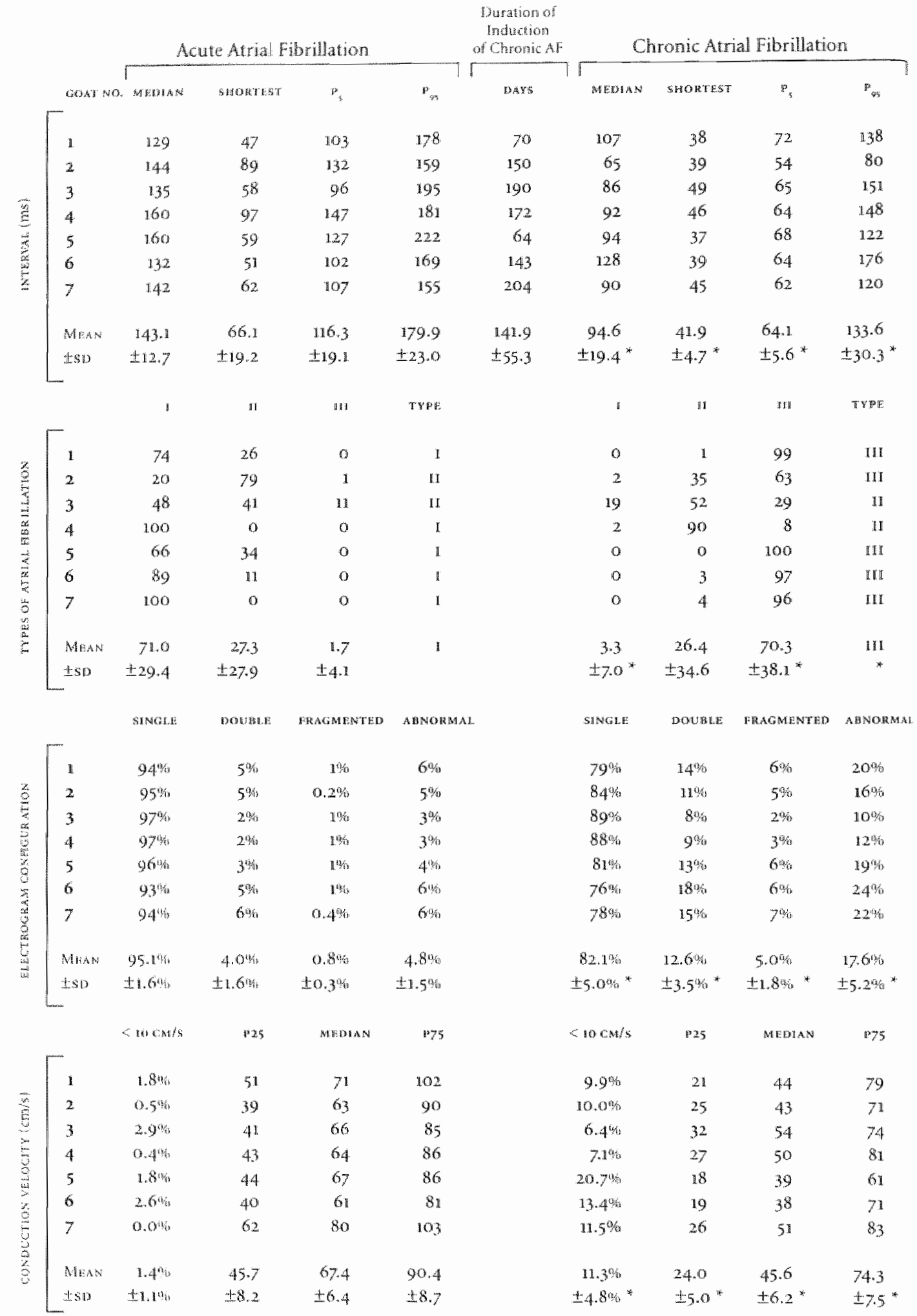


still had Type r ( 3 and 4). One goat did not show a change in the type of fibrillation. Nonetheless, even this goat demonstrated a marked increase in the degree of complexity of fibrillation, because the number of ype a beats decteased from $48 \%$ to $19 \%$ and the number of type un beats nearly tripled, from $11 \%$ to $29 \%$.

\section{Configurations of Fibrillation Electrograms}

In Figure 4.6, a typical map of chronic Af is given together with some recorded electrograms. Single potentials were recorded at sites where activation was homogeneous. Long-double potentials were seen at arcs of conduction block. Fragmented electrograms appeared at sites where conduction was slow or where a wavelet piwoted around the end of an arc of block. Thus, in the goat, both during acute and chronic atrial fibrillation a clear relation existed between the configuration of the fibrillation electrograms and the local spatial spead of activation.

The different degree of complexity during acure and chronic atrial fibrillation is also expressed by the proportion of abnormal electrograms (Table 4.1). During acute fibrillation, fragmented electrograms were rare $(0.8 \pm 0.3 \%)$ and also double potentials were only rarely seen $(4 \pm 2 \%)$. Single deflections representing fast uniform conduction were in the far majority $(95 \% \pm 2 \%)$. Although during chronic atrial fibrillation single potentials still dominated $(82 \% \pm 5 \%, P<.05)$, the proportion of abnomal potentials (long-double and fragmented) had increased from $4.8 \% \pm 1.5 \%$ to $17.6 \% \pm 5.2 \%$ ( $P<.0001)$.

\section{Conduction Velocity}

In the upper panel of Figure 4.7 conduction velocity histograms during atrial fibrillation $(n=7)$ are shown. The individual values are given in Table 4.1. The amount of abnormal conduction ( $<10 \mathrm{~cm} / \mathrm{s}$, indicating conduction block and $/ \mathrm{or}$ slow conduction) clearly increased from $1 \pm 1 \%$ during acute $A$ to $11 \pm 5 \%$ during chronic fibrillation $(p<.002)$, and more than $25 \%$ of the measurements $\left(p_{2}\right)$ became slower than $24 \mathrm{~cm} / \mathrm{s}$. The $\mathrm{P}_{2,}$ during acute Af was $46 \mathrm{~cm} / \mathrm{s}$ (decrease, $48 \%, 0<.05$ ). Actually, all conduction velocities below $30 \mathrm{~cm} / \mathrm{s}$ were significantly more frequently ocurting during chronic fibrillation ( $p<.002$ ), whereas during acute $\mathrm{AF}$ conduction velocitics between $60 \mathrm{~cm} / \mathrm{s}$ and $110 \mathrm{~cm} / \mathrm{s}$ dominated $(p<0.02)$. Also, very fast conduction $(>100$ $\mathrm{cm} / \mathrm{s}$ ) was fairly common cluring acute $\mathrm{AF}$, and more than $25 \%$ of measurements $\left(\mathrm{B}_{7}\right)$ were faster than $90 \pm 9 \mathrm{~cm} / \mathrm{s}$. During chronic fibrillation, such fast conduction occurred less frequently, and the $p_{75}$ was $74 \pm 8 \mathrm{~cm} / \mathrm{s}(-18 \%, p<.05)$. The median conduction velocity slowed down from $67 \pm 6 \mathrm{~cm} / \mathrm{s}$ to $46 \pm 6 \mathrm{cms} / \mathrm{s}(-31 \%, p<.05)$. 


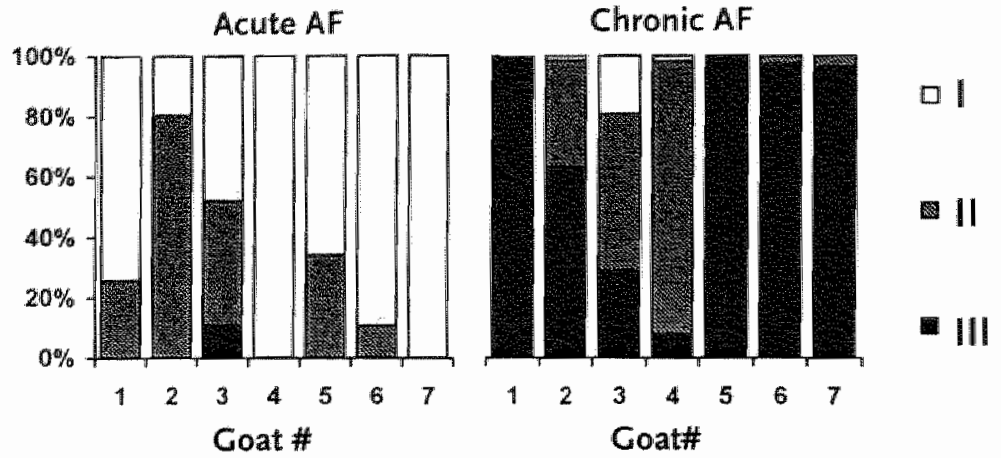

Figure 4.5

The degree of complexity during acute and chromic atrial fibrillation. The proportion of each type of beat (ordinate) is plotted for each individual goat (abscissa). During acute atrial fibrillation the proportion of type I beats (white areas) is significantly larger than during chronic AF in every goat. The proportion of type III beats (black areas) increases markedly during chronic AF in each individual. The proportion of type II beats (gray areas) changes in most goats when going from acute to chronic AF, but this varies greatly per individual and is not statistically sügnificant.

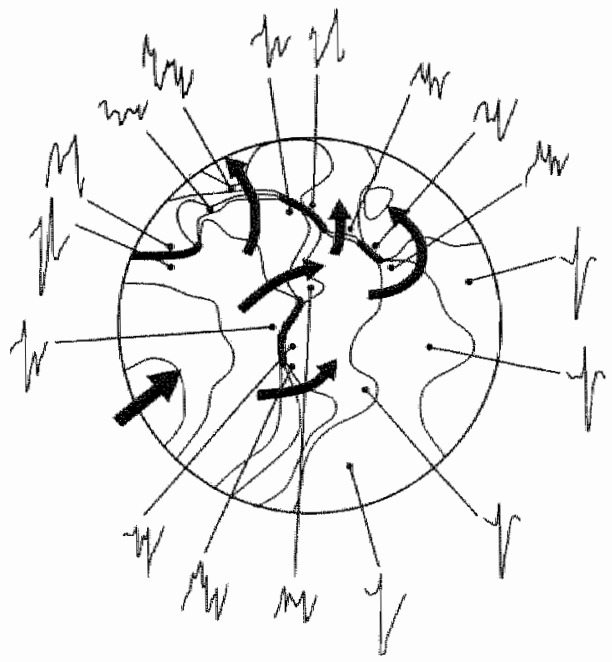

Figure 4.6

Figure 4.6: Activation map and electrograms of a type 1/1 beat during chronic Af in goat 4 . $150 c h r o n e s$ are drawn at 10-milliseconds intervals. Thick lines indicate conduction block, dashed lines indicate collision, and crowding of isochromes represents slow conduction. Arrows indicate the general direction of conduction. Several of the recorded electrograms are plotted around the map. Their recording sites are indicated by a thin straight line. See text for further description 

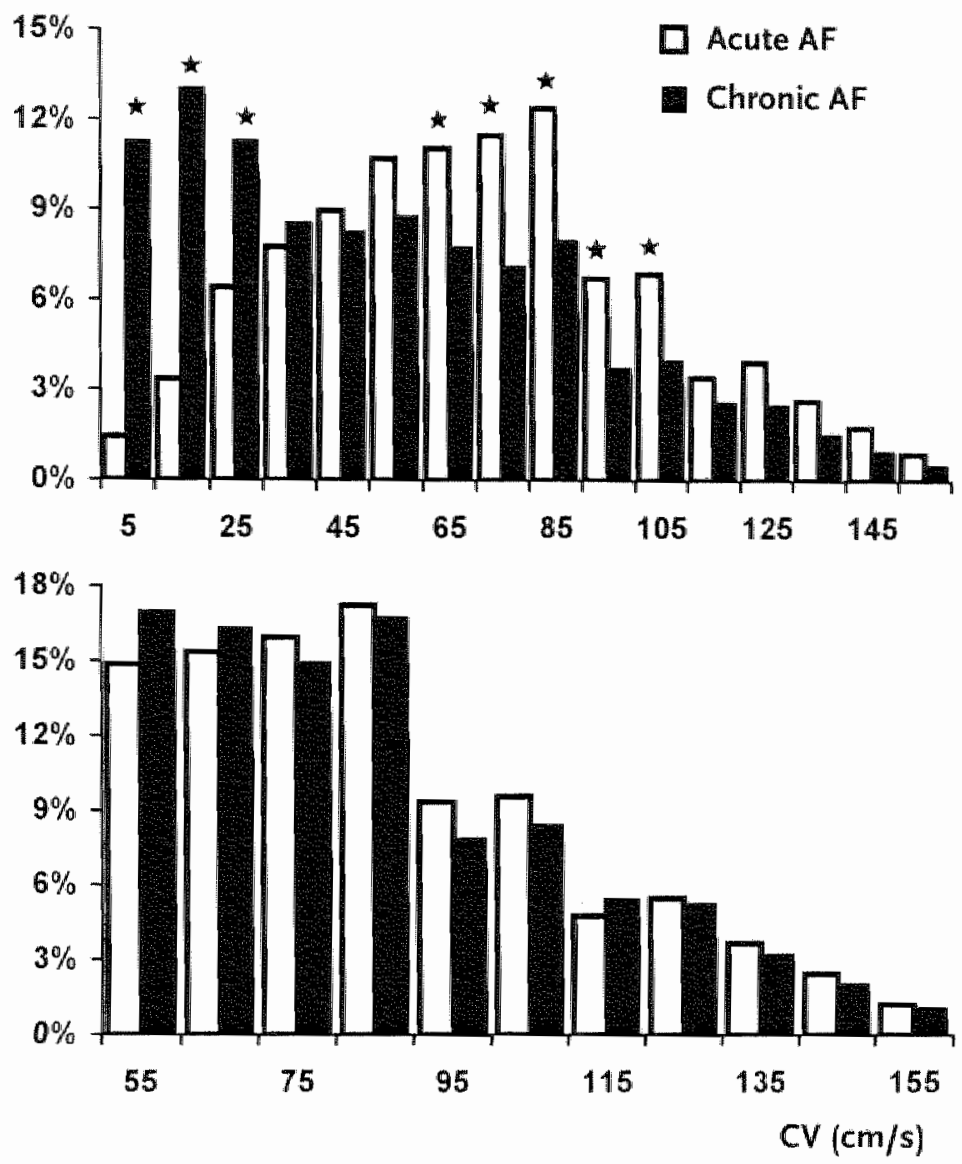

Intra-atrial conduction velocity histograms during acwte (white bars) and chronic atrial fibrilation (black bars) in the goat. For these histograms the measurements of every individual goat during 12 seconds of AF were summed. The percentage of measurements (ordinate) is plotted against the fibrillatory conduction velocity (in $\mathrm{cm} / \mathrm{s}$, abscissa). Stars indicate statistical differences between acute and chronic A.F $(P<.05)$. The upper panel shows the proportion of all conduction velocities. Median values were 67 and $46 \mathrm{~cm} / \mathrm{s}$, respectively. A totall of 205.574 measurements of conduction velocities were made. In the lower pantel, the distribution of only the conduction velocities $>50 \mathrm{~cm} / \mathrm{s}$ were plotted. See text for further description. 
To study conduction during atrial fibrillation in more detail, a separate plot was made of the distribution of farly normal conduction $(>50 \mathrm{~cm} / \mathrm{s}$, lower panel of Figure 4.7). This plot shows no sigmificant differences between acute and chronic atral fibrillation $(P>$. on). Other plots (conduction velocities $>40 \mathrm{~cm} / \mathrm{s},>30 \mathrm{~cm} / \mathrm{s}$, or $>20$ $\mathrm{cm} / \mathrm{s}$ ) still demonstrated slower conduction during chronic a compared to acute Af, but only the velocities below $50 \mathrm{~cm} / \mathrm{s}$ were more frequently ocsuring (not shown). Thus, the median conduction velocity during atrial fibrillation slowed down in the process of becoming chronic, but the difference lies only in the range of $0.50 \mathrm{~cm} / \mathrm{s}$, due to an overabundance of conduction welocities below $30 \mathrm{~cm} / \mathrm{s}$.

In order to test whether the observed slowing of conduction during chronic atrial fibrillation is due to a structural change in the conduction properties of the atria, conduction during regular atrial activation (sinus rhythm and several pacing rates) was measured. During the acute experiments, this was performed before atrial fibrillation was induced. When atrial fibrillation had become chronic the measurements were made five minutes after electrical cardioversion of AF. In Figure 4.8 , maps are shown for all goats during regular pacing (interval, 400 milliseconds), are shown both before and after chronic atrial fibrillation. All beats were conducted homogeneously. In some goats the conduction velocity during the acute experinent was a little faster than after chronic Af had been induced $(2,3,5$, and 7 ), but in other goats this was the opposite (goat 1,4 , and 6). On the average the conduction velocity during pacing at 400 -millisecond interval was not altered by 20 weeks of $A P(P>.05)$. Figure 4.9 shows the rate related conduction velocity during atrial pacing. After chronic A $F_{n}$ the average conduction velocities wert slightly faster than during control, but this difference was not statistically significant. Apparently, long-standing atrial fibrillation (142455 days) does not reduce the structural or functional conduction properties of the goat atria.

In Figure 4.9, the conduction velocities of uniformly conducled beats during atrial fibrillation are indicated in the curves (arrows). During acute atrial fibrillation uniform conduction was significantly taster $(76+6 \mathrm{~cm} / \mathrm{s})$ than during chronic Af $(56 \pm 5 \mathrm{~cm} / \mathrm{s}, \mathrm{p}<.05)$. However, this difference could be explained by the difference in median fibriliatory cycle length ( $43 \pm 13$ milliseconds and $95 \pm 19$ milliseconds, respectively), as these values fit into the rate-related welocity curves. Apparently, even during chronic atrial fibrillation, the tissue is still capable of conducting at nomal speed. Table 4.2 summarizes the individual uniform conduction velocities in the acute and chronic experiments. 

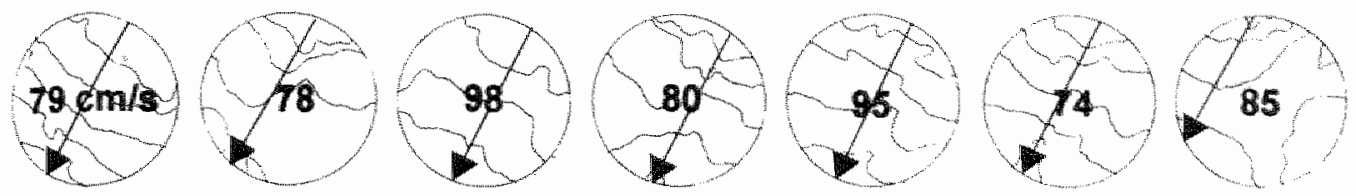

AF 7o days

150 days

190 days

172 days

64 days

143 days

204 days
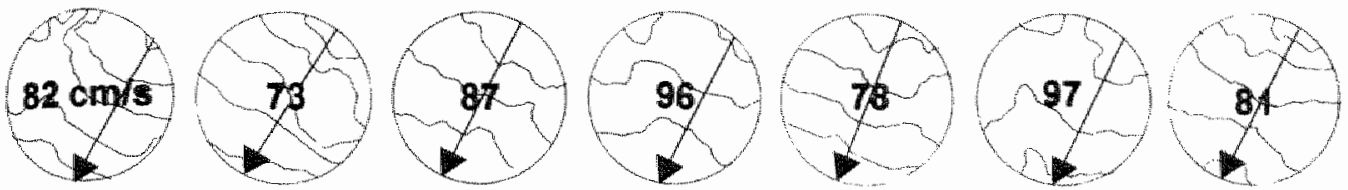

Intra atrial conduction during pacing at 400 -millisecond interwal in comtrol measurements (upper panels) and after conversion of chronic Af (lower panels). Pacing was performed at the right side of the Bachmann's bundle. for exery individual goat a representative beat is shown. Isochrones are drawn at 10-millisecond irtervals, and arrows, indicate the direction of activation. The uniform conduction velocity for each beat is plotted in the maps. The duration of continuous Af induction is shown between the maps. See text for further description.

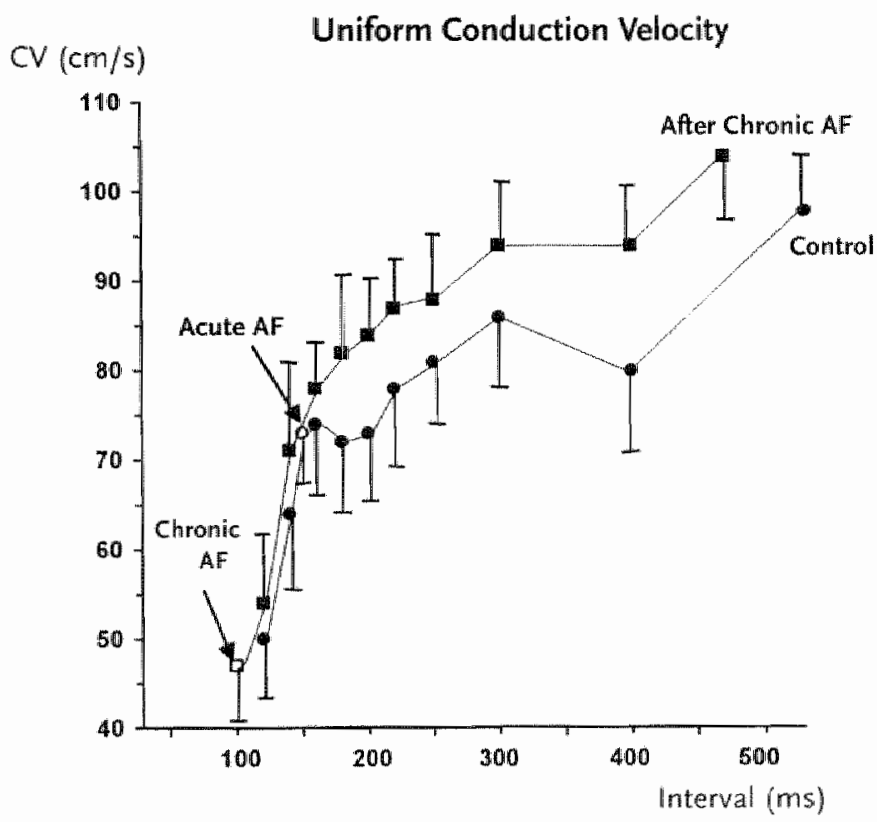

The awerage uniform conduction velocity during sinus rhythm, pacing at intervals beiween 120 Figure 4.9 and 400 milliseconds, and atrial fibrillation. The experiments were performed during control and after conversion of chronic AF. The last value of each curve is during sinus rhythm. The average sinus interval changed from $577 \pm 56$ milliseconds to 46740 milliseconds $(p<05)$ Arrows indicate the uniform conduction velocities during acute $\mathrm{F}(\mathrm{O})$ and chromic fibrillation (a). See text for further description.

Figure 4.8 


\section{Estimation of Reentry Pathlength}

Assuming that atrial fibrillation is maintained by reentry, we estimated the average reentry pathlength during atrial fibrillation by multiplying the median conduction velocity by the median cycle length. In Figure 4.10 the estimated pathlengths during acute and chronic atrial fibrillation are plotted for all seven goats. During acute AF, the estimated pathlength ranged between 8 and $12 \mathrm{~cm}$ (average $9.7 \pm 1.2 \mathrm{~cm}$ ). During chronic fibrillation, the assumed reentry pathlength was much shorter, ranging between 3 and $5 \mathrm{~cm}$ (average $4.3 \pm 0.8 \mathrm{~cm}, p<.05$ ). There was no overlap between the two populations, suggesting that atrial fibrillation does not become chronic until the average reenty pathlength has become shorter than $5108 \mathrm{~cm}$.

Duscussion

\section{Comparison of Acute and Chronic Atrial Fibrillation}

There are several indications that the electrophysiology of acute atrial fibrillation is different from chronic AF. Chronic fibrillation is notoriously more difficult to convert to sinus rhythm, ${ }^{13.1+144}$ and is commonly associated with structural cardiac disturbances. ${ }^{75}, 133,145$ It has been shown that chronic Af alters the structure of atrial cardiomyocytes. ${ }^{152-454}$ Differences in the electrophysiological behavior of acute and sustained $A F$ have been demonstrated in the study of Wiffels et all. ${ }^{130}$ The fibrillatory rate increased markedly during the time course of induction of chronic AF, refractory periods shortened significantly, and the physiological adaptation of the refractory periods to heart rate was lost or became inverted. Conduction velocities during fast regular pacing were slightly higher after 2-4 days of continuous Af induction than during control, while there was no increase in the spatial dispersion of refractory period or fibrillatory intervals. The estimated wavelength during Af decreased from $8-9 \mathrm{~cm}$ to $5-6 \mathrm{~cm}$.

Reduction of both the refractory periods and the physiological adaptation to heart rate by atrial fibrillation was demonstrated in several studies. ${ }^{130.148,455.595}$ This process of long-term adaptation of the atria to the rapid rates is called electrical remodeling. ${ }^{\text {six }}$ It has been shown to be diminished by calcium blockade, ${ }^{\text {ito }}$ and is associated with a reduction in the $\mathrm{L}-\mathrm{type} \mathrm{Ca}^{21}$ and transient outward current. ${ }^{15 *}$

\section{Activation Patterns and Electrogram Morphology}

This study shows that the activation patterns during chronic atrial fibrillation are 


\section{Estimated Reentry Pathlength}

\section{$\mathrm{CV}(\mathrm{cm} / \mathrm{s})$}

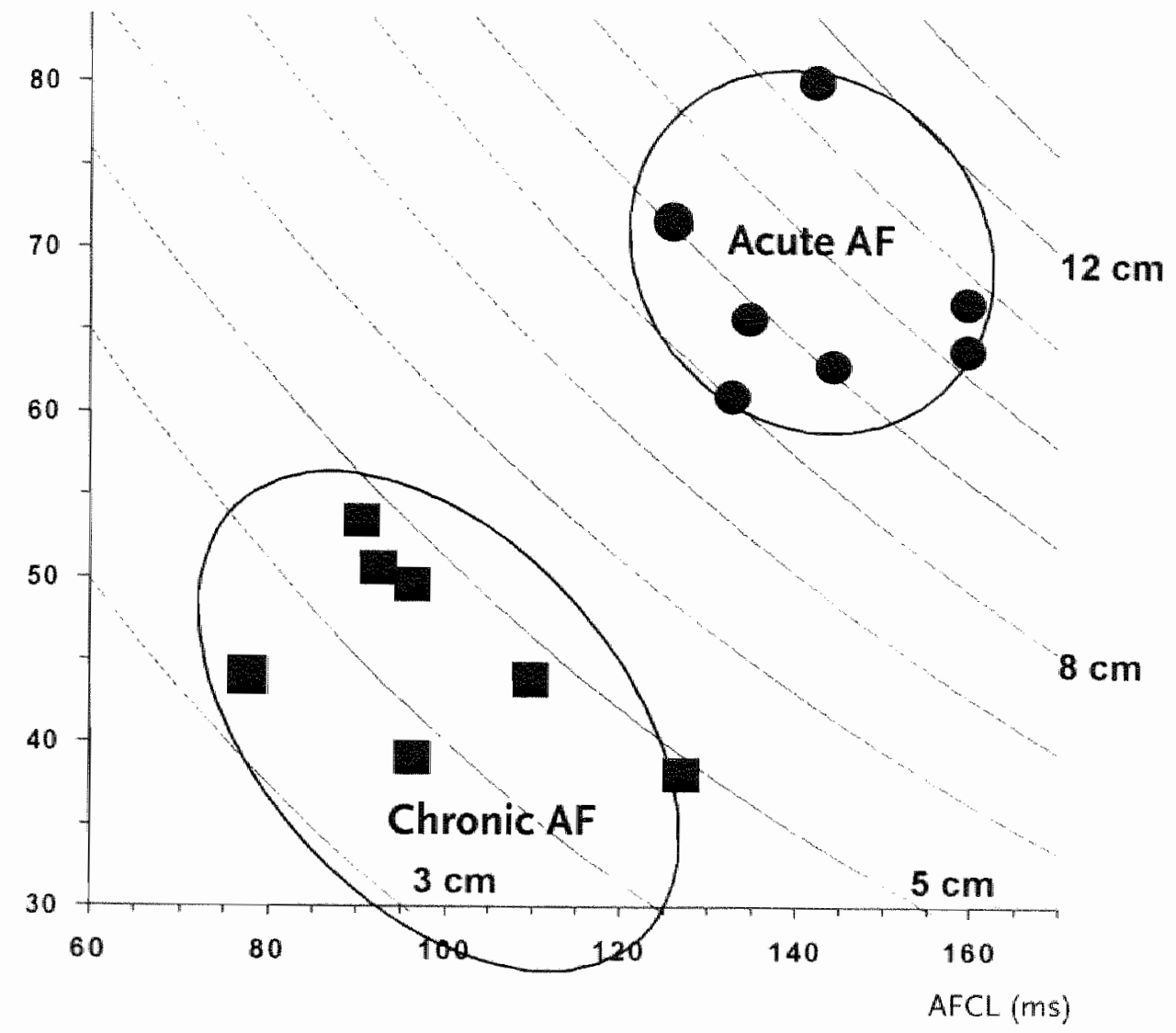

Median reentry pathlengths during acute and chronic atrial fibrillation an on the ordinate the median conduction velocity during atrial fibrilltation (AFCY) is plotted, and on the abscissa the median fibrillation interval (AFCl). The associated pathlengths (in $\mathrm{cm}$ ) are indicated by curved lines. See text for further description. 
considerably more fragmented than during acute fibrillation. In a previous study in humans, we have shown that the degree of complexity of atrial activation is strongly related to the rate of atrial fibrillation. ${ }^{\mathrm{s}}$. The fact that the fibrillatory cycle lengths during chronic fibrillation was considerably shorter than during acute Af is consistent with this. The interindividual variation in the type of fibrillation was larger in humans than in goats. Most goats $(71 \%)$ had type I fibrillation in the acute experiment and none demonstrated type In activation. In humans, acutely induced atrial fibrillation was of type II in $28 \%$, although type I Af was most common $(40 \%)$. Possibly, there is a difference between species. However, it is allso conceivable that this difference is caused by differences in the atrial substrate. Humans with a wpw-syndrome might have had a certain degree of abnormal cardiac function, whereas the goats had normal atria at the start since they had been in normal sinus rhythm from birth.

Conduction disturbances like conduction block and slow conduction, can be identified by the presence of double and fragmented electrograms. ${ }^{151}$ In this study, after induction of chronic $\mathrm{AF}$, the amount of apparently slow conduction $(<10 \mathrm{~cm} / \mathrm{s})$ was increased to the same amount as the percentage of abnormal electrograms ( $+10 \%$ and $+13 \%$, respectively). The actual percentage of abnormal electrograms was larger than conduction $<10 \mathrm{~cm} / \mathrm{s}$, both during acute $(5 \pm 2 \%$ versus. $1.4 \pm 1.1 \%)$ and chronic Af $(18 \pm 5 \%$ ws. $11 \pm 5 \%)$. It was similar to the incidence of conduction $<20 \mathrm{~cm} / \mathrm{s}(4.6 \pm 1.1 \%)$ during acute $\mathrm{AF}_{\mathrm{r}}$ and during chronic AF $(19.1 \pm 4.3 \%)$. Thus, abnormal electrograms arise at conduction velocities lower than $20 \mathrm{~cm} / \mathrm{s}$. This explains why even during the highly complex activation patterns of chronic atrial fibrillation only $18 \%$ of the electrograms were abnormal.

During chronic atrial fibrillation it appeared to be more common that wavelets arose somewhere in the middle of the mapping area. Often this did not result in a completely focal pattern of activation, since the impulse was blocked in one direction or immediately collided and/or fused with another wavelet. It remains unclear whether these "foci" are the result of the increased degree of fragmentation, facilitating wavelets to conduct in deeper layers or over trabeculae (epicardial breakthrough), or whether they indicate abnormal automaticity. A considerable incidence of "focal" patterns of activation has also been reported in the right atria free wall in patients with chronic a F. ${ }^{12}$

\section{Does Atrial Fibrillation Depress Atrial Conduction?}

In the present study there was no significant effect of chronic atrial fibrillation on 


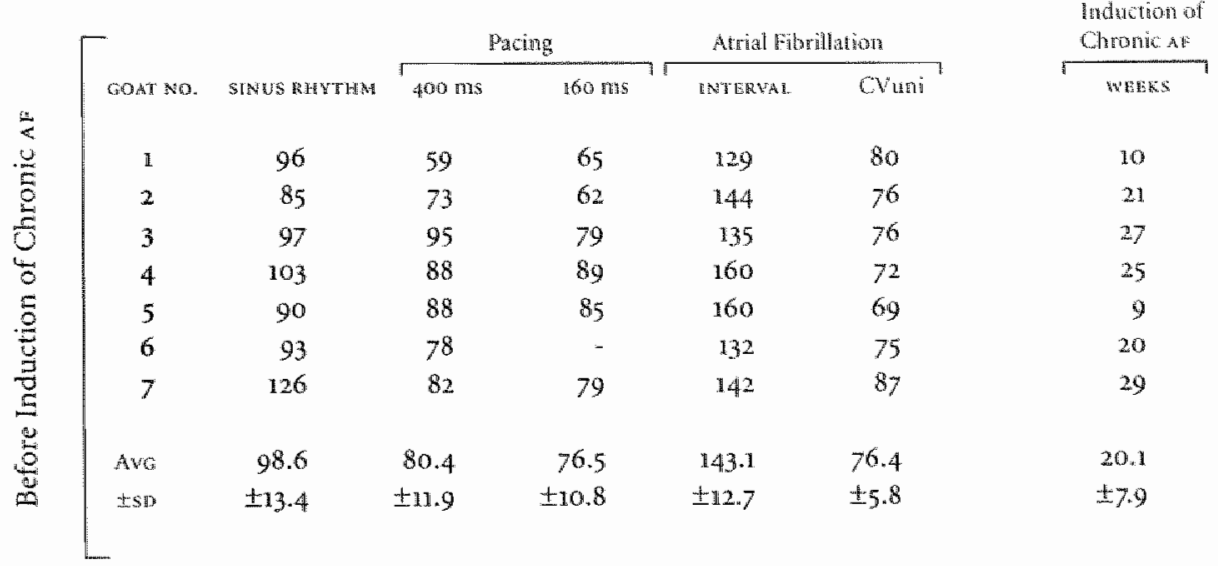

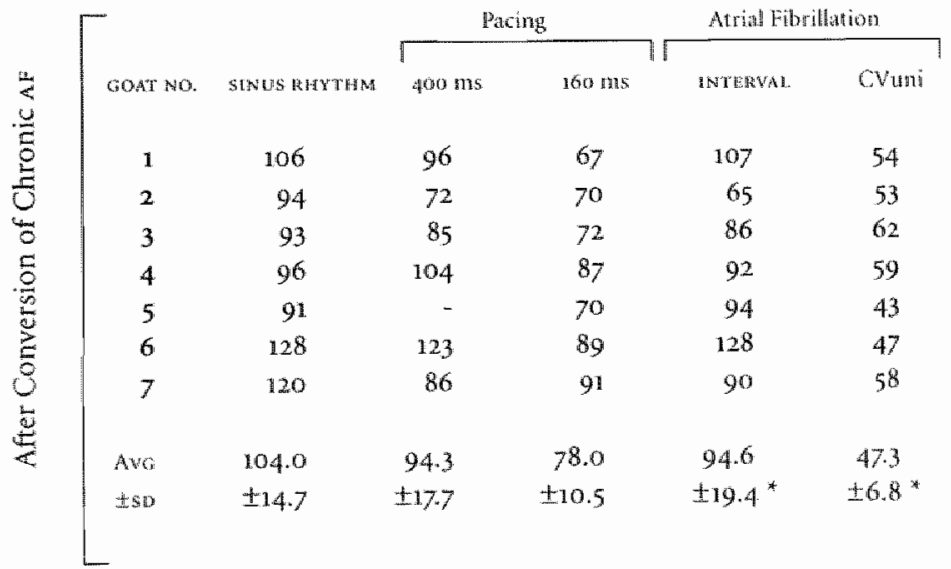

Af indicates atrial fibrillation; Interval, median fibrillation anterval in millisaconds; CVuni, conduction

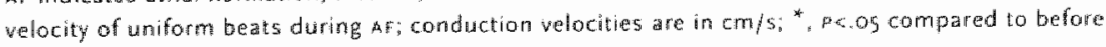
induction 
conduction velocity during uniform atrial activation (Table 4.2 and Figures 4.8 and 4.9). Actually, the rate-related velocity curves after chronic af were $9 \pm 6 \%$ faster than before $A$. This difference is in accordance with the slight increase in conduction velocity reported by Wijfels et al. ${ }^{130}$ During atrial fibrillation, the conduction velocity of uniformly conducting waves was rate related (Figure 4.9). Furthermore, in the range of conduction velocities $>50 \mathrm{~cm} / \mathrm{s}$ no differences were observed between acute and chronic atrial fibrillation. Only conduction velocities below $50 \mathrm{~cm} / \mathrm{s}$ were different, due to a surplus of conduction slower than $30 \mathrm{~cm} / \mathrm{s}$ during chronic Ap (Figure 4.7). This surplus might be explained by an increase in the number of wavelets, since the chance that a wavelet encounters tissue that is still (partially) refractory from activation by another wavelet increases accordingly. "Whus the chance for conduction block or slow conduction also increases. One might such conduction disturbances physiological, rather than functional or structural.

These findings are in contrast to the results of a recent study in dogs that were permanently paced (at 150-millisecond interval, for six weeks) in order to induce sustained atrial fibrillation. ${ }^{35}$ In the latter study conduction was reported to be significantly depressed from $110 \pm 5 \mathrm{~cm} / \mathrm{s}$ to $82 \pm 4 \mathrm{~cm} / \mathrm{s}$ after long-term rapid pacing $(-25 \%, p<.01)$. The differences between these and our findings are puzzling. It might be caused by a difference between species, possibly due to differences in atrial size. Also, diferences in the model might be relevant. In our chronic goat study, AF is inmediately reinduced by burst pacing after fibrillation has stopped. in line with the progressive increase in the duration of af episodes, the period between bursts progressively increases. This is not the case in the dog model. Constant application of electrical pulses at twice the threshold might have a different effect on atrial conduction than the permanent presence of atrial Vibrillation.

The different effects on conduction in the chronically fibrillating goat model and the chronic atrial tachycardia dog model might be partially explained by differences in degree of atrial dilatation. Measurements of conduction velocity can be intluenced by atrial dilatation. If atrial size were to increase, the number of calls under a reconding plaque with a fixed interelectrode distance would decrease accordingly. When cell-to-cell conduction remains similar, it might introduce a patadoxical increase in conduction velocity. Unfortunately, in the present study the atrial dimensions were not monitored. However, it has been shown that the atrial diameter during chronic atrial fibrillation is about $8-15 \%$ larger than in 
individuals with acute atrial fibrillation. ${ }^{16 !-164}$ Assuming a similat increase in atrial diameter in our study might explain, why after chronic af the conduction velocities during regular activation were consistently $9 \pm 6 \%$ faster than before induction of atrial fibrillation. This would then indicate that actual cell-to-cell conduction remained more or less the same. However, it might be more relevant to compare the degree of atrial dilatation during chronic $\mathrm{A}$ to healthy individuals instead of patients with paroxysmal atrial fibrillation, since the goats had been in sinus rhythm from birth. Groupstudies by Shapiro in thumans ${ }^{\text {its }}$ and $\mathbb{E}$ ivan ${ }^{150}$ and Morillo ${ }^{153}$ in dogs, show that compared to normal indiwiduals the atrial diamerer during chronic $A \mathrm{~F}$ is increased by approximately $25 \%$. This would indicate that the cell-to-cell conduction in our chronic goat model was actually depressed by approximately $16 \%$.

Changes in conduction velocity by chronic atrial fibrillation might be caused by differences in the number of gap junctions. In the atria connexin 40 and 43 are the most specific gap junction proteins. ${ }^{16 \pi} 169$ Several studies have shown that conductance between neighboring cells increased with the number of gap junctions. ${ }^{170-172}$ of Elvan ett al. ${ }^{156}$ demonstrated that the density of connexin 43 doubles in dogs with pacing-induced sustained AF. However, they state that: "gap junctions, though increased, may not be normal. Further, an increase in gap junctions and/or a change in distribution may impair orderly impulse propagation and prolong conduction times." In another study they showed that the intra-atrial conduction time was prolonged during pacing-induced chronic AF. ${ }^{1 / 3}$ However, the relation with gap junctions was not investigated. At this point it remains unclear, whether during atrial fibrillation the number of gap junctions actually decreases or increases and whether this improves or impairs cell-to-cell conduction. This might be studied further in the various models of chronic atrial fibrillation and in patients.

\section{Atrial Fibrillation Reduces the Size of Reentry Circuits}

In a study of Rensma et al. ${ }^{\text {ton }}$ it was shown that the critical wavelength for induction of atrial fibrillation is $8 \mathrm{~cm}$. At a wavelength zone between 10 and $12 \mathrm{~cm}$, spontaneous repetitive responses occurred, and abowe a wavelength of $12 \mathrm{~cm}$ no arrhythmias could be prowoked. The estimated reentry pathlength (conduction velocity $x$ fibrillation interval) is per definition longer than the wavelength (conduction velocity $x$ refractory period). Our estimation of the pathlength suggest that there is a critical pathlength for atrial fbrillation to become chronic. 
No values beween 5 and $8 \mathrm{~cm}$ were found. Possibly this discontinuty is bridged during the period that Af episodes progressively increase in duration, but still stop spontaneously.

The average pathlength during acute $A$ (10t1 $\mathrm{cm})$ would require a surface area of approximately $8 \mathrm{~cm}^{2}$ for reentry; during chronic $\mathrm{AF}(4 \pm 1 \mathrm{~cm})$ this would be 1.5 $\mathrm{cm}^{2}$. This indicates that during chronic at $\mathrm{F}$ at least five times more wavelets would fit into the atria than during acute $A$. This is in accordance with the single wavelets recorded during acute fibrillation in the right atrial free wall, and the much higher number of wavelets during chronic af (not quantified, but often between four and six). The total number of wavelets must be the sum total of this, since we mapped only a segment of the whole atria. The abundance of wavelets explains the great stability of chronic atrial fibrillation. Arrest of several wavelets does no longer lead to termination of atrial fibrillation, since sufficient wavelets remain to perpetuate the arthythmia.

The study of Wijffels et al. ${ }^{130}$ showed that already after 24 hours of AF the fibrillatory cycle length was markedly reduced (by 26\%), while the conduction velocity remained the same. Thus the pathlength would have been reduced by $26 \%$, which allows approximately twice as many wavelets in the atria. Nonetheless, atrial fibrillation still stopped spontaneously, and episodes of Af lasted only a few minutes. After chronic Af was induced, the interval was shortened only slightly further (by 6\%), and the conduction velocity during pacing was still unaltered. Therefore, they assumed that the pathlength (and the number of wavelets) was onlly slightly higher than after 24 hours and they postulated that other mechanisms had to play a role. However, since during chronic $\mathrm{Al}$ the conduction: velocity remains rate related, a small decrease of the fibrillatory interval might further decrease fibrillatory conduction velocity, and have a considerable effect on the fibrillatory pathlength. Thus the critical pathlength for chronic atrial fibrillation might be exceded. Other factors might still be necessary to induce chronic An, but it seems worthwhile to further investigate the time course of reduction of fibrillatory pathength.

\section{Limitations of the Study}

The population of the present study group is not representative for the group of patients with clinical paroxysmal or chronic atrial fibrillation. It has been demonstrated that age ${ }^{4,127}$ and underlying heart disease ${ }^{33,145}$ are strongly correlated with the incidence of atrial fibrillation. Therefore, the mechanisms 
underlying clincal af may be different from the electrically induced acute and chronic atrial fibrillation as studied in the goats. The question also remains whether the mechanism of atrial fibrillation is similar for humans and goats, and if so, whether the process of atrial fibrillation propagating atrial fibrillation is similar. Another important limitation of this study is that chronic atrial fibrillation was induced after opening of the pericardium and chronic implantation of epicardial electrodes. However, atrial contact was always good and no areas of structural conduction disturbances were found.

In this study measurements were only made at the right atrial free wall during 12 seconds of atrial fibrillation. Therefore, conclusions cannot be drawn about other parts of the atria and other episodes of atrial fibrillation. Since the amount of atrial dilatation was not measured, it is difficult to interpret the measurements of conduction velocity during chronic atrial fibrillation.

\section{Clinical Implications}

The present study demonstrates that the stability of chronic atrial fibrillation is associated with a larger number of reentrant wavelets. This might be of importance for drug treatment of atrial fibrillation. The chance for successful chemical cardioversion and/or prevention of atrial fibrillation might be affected by the degree of complexity of atrial fibrillation, and thus by the size of reentrant circuits. It has been shown that the class In drug Ibutilide, was significantly more effective in converting atrial fibrillation when the fibrillatory rate was slower. It was suggested that the drug was more effective in fibrillation with fewer wavelets. ${ }^{174}$ It seems likely that drugs that increase wavelength, may not be effective in atrial fibrillation in which the wavelength is so short that a percentual increase still allows a multitude of wavelets to remain in the atria. The type of fibrillation may therefore differentiate between patients with different therapeutic profiles. At this moment, however, these implications are still speculative and more information is needed about the effect of drugs in different types of fibrillation, before any firm conclusion can be drawn. 



\section{Chapter 5 | General Discussion $\mid$}

"No longer do I wish to be excited, stimulated; ny own heart storms enough in itself.(.)

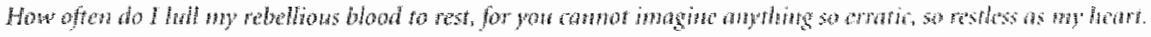

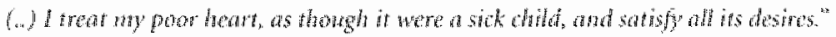


The mechanism of atrial fibrilltion has been puzzling investigators and clinicians a long time (Chapter 1). The present thesis describes the activation patterns during electrically induced atrial fibrillation (AF) in w $P$-patients and chronically instrumented goats. The aim of the thesis was to characterize the fibrillatory activation of the atria in humans, and to investigate the differences between acute and chronic $A F$, in order to reveal some details of the mechanism. Previous work during Af in dogs ${ }^{5 * 406}$ had indicated that the activation of the atria during fibrillation is based on reentry. A spectrum of activation patterns was seen, varying from single circuits to mulciple wavelets wandering around natural anatomical obstacles and functional conduction blocks. In humans only few studies were available ${ }^{5 *_{*}}$ and no systematic analysis of the activation patterns during human atrial fibrillation had been done.

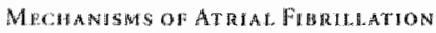

The observation multiple wawelets were simultaneously present in many of the study subjects presented in this thesis, suggest a role for the multiple wavelet hypothesis as a perpetuating mechanism during atrial. fibrillation in humans and goats. This bypothesis describes a now widely accepted mechanism of atrial fibrillation. However, other electrophysiological mechanisms may as well be involved, explaining some of the varieties of activation that we obserwed. Also, spontaneous Af in patients might involwe different mechanisms involved than the electrically induced atrial fibrillation that we studied.

The different mechanisms with their therapeutical options are summarized here and illustrated in Figure 5.1. A) Multiple wawelets, perpetuating independently from whichever possible initiating agent. ${ }^{4,4}$ Artest of one of such wavelets would hardly have any effect on perpetuation of the arrhythmia. To stop atrial fibrillation a method has to be sought that minimizes the number of wavelets, or makes it impossible for new wavelets to generate and reenter.

B) A mother wave that revolwes faster than the surrounding tissue and, can give rise to passive daughter waves that result in a highly irregular activation pattern in the remainder of the atria. ${ }^{54}, 1 \%, 1 \%$ If this mother wave could be dissolved, the atrhythmia would stop.

c) Automatic on triggered foci which fire at a high rate. A complex activation pattern arises when of such impulses block at sites that are in a refractory state. Since the activation of the atria is dependent upon the focal impulses, arrest of the foci 
would bring the atria to rest. 180.135

D) "Three dimensional atrial fibrillation", in which the multiple wavelets have become so small that they pass through in several layers of the atrial tissue and through the free rumming endocardial trabeculae. Thus, the activation pattern is highly fragmented with a considerable amount of "focal" epicardial brakhrough patterns. The mechanism would seem similat to that of ventricular fibrillation and be equally stable.

The possible role of these different mechanims during atrial fibrillation in humans and goats will be discussed in the following sections.

Mapping of Atrial. Fibriliatron

\section{General Activation}

In all instances of atrial fibrillation we found an atrial rate that was $4-6$ times faster than during sinus thythm. This resulted in electrocardiograms that were characteristic of atrial fibrillation. The atrial activation was too fast and uncoondinated to elicit $p$-waves. The ventricular tate was irregular and slower than the atrial rate. Atroventricular syncronicity was dearly absent (Chapters 2-4).

\section{Local Unipalar Electrograms}

The local electrograms during atrial fibrillation were much more irregular than during sinus rhythm and rapid pacing. Long-double potentials and tragmented ellectrograms (abnormal potentials) wete extremely rare during sinus thythm and rapid pacing in both humans and goats $(<1 \%)$, whereas they were nuch more common during fibrillation (15\% in humans, $5 \%$ during acute Af in goats, and $18 \%$ during chronic AF in goats) (Chapters 3 and 4 ). Fast, uniform conduction was shown to be present at sites where the electrogram exhibited a single sharp deflection. Arcs of conduction block were marked by double deffections in the electrogram, the rime between the wo deflections representing the difference in activation time at both sides of the block. Double deflections with a tince difference of 10 milliseconds on less, were also found at areas where wo on more wavelets collided. And finally, areas of slow conduction and piroting points at the end of an arc of block were identified by the occurrence of fragmented complexes in the local electrograms. However, it was demonstrated that in our patients these conduction disturbances were physiological rather than the result of anatomical abnormalities (Chapter 3 ). 


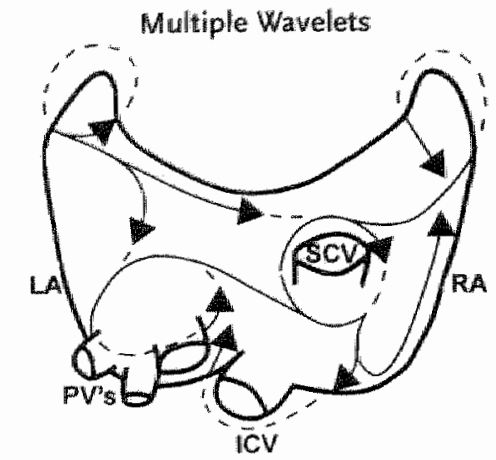

Mother Wave - Daughter Waves

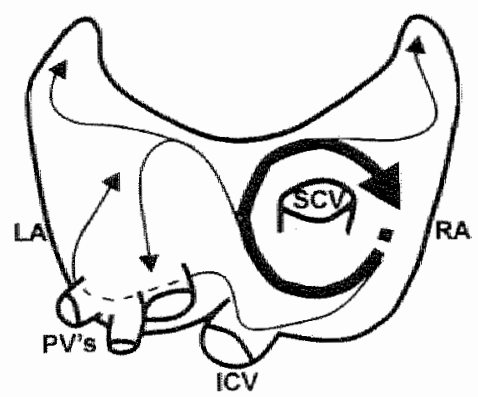

Focal Activation

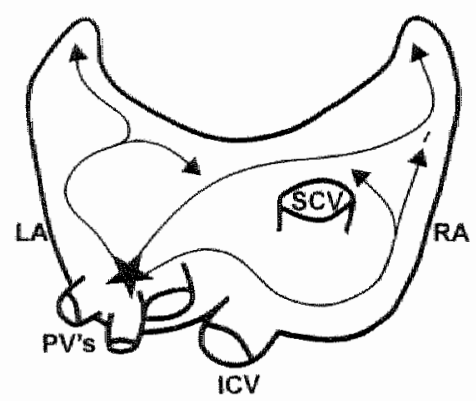

Three Dimensional Atrial Fibrillation

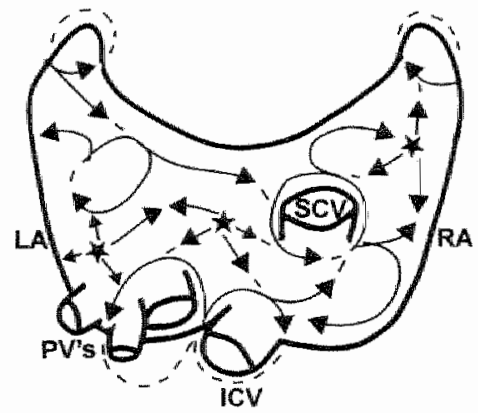

Figure 5.1

Mechanisms of atrial fibrillation. Diagrams of the heart in which the left and right atria are erlarged for clarity. Multiple wavelets: arrows indicate wavelets randomly reentering into tissue that was previously activated by itself or by another wavelet. One must inagine that the rolle which wavelets travel can vary every beat. Mother wave - daughter waves: the thick arrow indicates the mother wave, thin arrows the daughter waves. The mother wave is often fixed to an anatomical obstacle, while the daughter waves can perpetuate through all excitabie tissue. Focal activation: the star indicates the focus, which often lies within the region of the pulmonary veins. The resulting wavelets can be random and different every beat. Three dimensional atrial fibrillation: stars indicate epicardial breakthrough of wavelets, that have been conducting in a lower layer or over an endocardial trabecula. Reentrant circuits may turn around functional lines of conduction block or around an anatomical obstacle. Every beat is different, depending on excitable cells. Actually, this is not a separate mechamism, but multiple wavelet reentry on a very small scale. RA indicates the right atrium; LA, left atrium: SCw, superior cawal wein, ICW, inferior caval wein; Pv's, pulmonary veins. 


\section{Activation Patterns}

The epicardial activation patterns that were reconstructed by high density mapping demonstrated that during atrial fibrillation one or more wavelets were simultaneously present in the right atrial free wall (Chapters 2-4). The varieties in the degree of complexity of activation in individual patients were described and classified (Chapter 2). In (4.0\%) of the patients, the right atrial free wall was dominantly activated by a broad single wavelet that conducted more or less uniformly. This pattern of activation was named type if fibrillation. In other patients (28\%), the activation maps were completely different. Most of the time their atria were activated in a highly complex fashion by three or more wavelets, which often conducted wery slowly through parts of the tissue and were separated by arcs of conduction block (type ur fibrillation). An intermediate pattern of activation (type 11), consisting of irregular conduction by a single wavelet or the simultaneous presence of two wavelets in the right atrial free wall, was seen in $32 \%$ of patients. However, since the degree of complexity increased gradually, the different types of fibrillation should not be regarded as separate entities but as an illustration of variations. The type of fibrillation bore no relation to the patients' characteristics or the duration of the episode. However, this classification might be associated with the clinically observed differences between flutterfibrillation, ${ }^{34,58}$ and coarse- and fine fibrillation. ${ }^{76 \cdot 73}$

\section{Local Conduction Disturbances}

In most cases, these wavelets turned around lines of functional concluction block, and propagated through areas where conduction was very slow $(<7.5 \mathrm{~cm} / \mathrm{s})$. The wavelets commonly collided or fused, or reentered into tissue that was previously activated by itself or another wavelet. The presence of such conduction disturbances is important for perpetuation of atrial fibrillation. An arc of block forces wavefronts to split into separate wavelets, and causes dissociation in conduction times. Thereby block introduces an inhomogeneous recovery of refractoriness, and even variations in the refractory period at closely adjacent sites. When wavelets can pivot around an arc of block the chance for reentry whether it is of the leading circle type or random, is increased. Again this increases the chance of perpetuation. Local slow conduction contributes to perpetuation of atrial fibrillation by increasing the conduction time over a short distance and thus shortening the wavelength. Collision is a common feature of atrial fibrillation when multiple wavelets are present. However, collision does not 
contribute to perpetuation of atrial fibrilation. In contrast, it leads to termination of wavelets. The source of atrial fibrillation must lie elsewhere.

\section{Reentry}

In our study in the wPw patients we made a distinction between perpetuation of $A$. by closed loop reentry and random reentry (Chapter 2). During closed loop reentry, a wavele follows more or less its own track and the interval is mone or less similar at all sites within the circuit. This is not the case during random reentry, when the tissue is activated in a different direction than it followed previously. The type of reentry does not necessarily indicate different mechanisms of perpetuation of atrial fibrillation. The simultaneous presence of multiple wavelets causes the wavelets to interfere with each other, leaving it to a matter of chance whether a wavelet will reenter itself or into tissue that was previously activated by another wavelet. Yet the difference is relevant because it bears consequences for the presence of an excitable gap. A wide excitable gap decreases the likelihood for wavelets to run into refractory tissue, and the tendency to divide around an area of functional block therefore might be diminished. Thus, the number of newly generated wavelets may decrease. Also, a wide excitable gap may lead to recovery of a larger part of the atrial tissue, promoting uniform. activation. Beat-to beat variations from complex to more or less uniform. activation might be caused by wariations in the excitable gap.

During random reentry, the interval increases gradually from the first point of reentry to more distant sites (Chapter 2, Figure 2.10). These distant sites may have recovered their excitability in the mean time, and thus an excitable gap may be introduced.

Also in closed loop reentry a short excitable gap can be present. The wavefront rotates around a central are of functional conduction block. It is one of the criteria for leading circle reentry that the revolution time is determined by the refractory period of the tissue in which the circuit rotates, and that no excitable gap is present. However, factors such as the curvature of the waveront play an additional rols. Often a wavefront makes a sharp u-turn at the pivoting point. Because the number of cells at a piwoting point is small, the excitatory current generated can be low. The excitatory current becomes even lower when the coupling between fibers is low, as can be found between longitudinal fibers and in aged hearts. In these cases, the wavefront can conduct only very slowly at the pivoting point or is unable to make the turn at that point. When the wavefront 
does not turn, it is forced to propagate forward, thereby lengthening the arc of block, until it manages to piwot. The revolution time has then become longer than the refractory period, and an excitable gap occurs. Both slow conduction and lengthening of the line of block lead to the introduction of a short excitable gap. $185.88 \%$

During fibrillation the atrial activation is evidently abnomal and apparently complex. It was shown that local conduction disturbances play an important role in the perpetuation of atrial fibrillation. Howewer, the amount of conduction disturbances varied greatly between individuals with equally stable atrial fibrillation. Classification of the degree of complexity, or the amount of conduction disturbances, offered a useful tool for investigation of the mechanism of atrial fibrillation.

\section{Different Types of Atrial Fibrillation; Are Different Mechanisms Involved? Types II and III Fibrillation}

Obviously in cases where two or more wavelets were simultancously present in the right atrial free wall (type $n$ and type In; Chapter 2, and chronic af; Chapter 4) the multiple wavelet theory $y^{\mathrm{B}}$ offers a likely explanation for the mechanism of perpetuation of atrial fibrillation. First of all, these are exactly the multiple wavelets that we observed. The frequent observation of both random reentry and dosed loop reentry within the boundaries of the right atrial free wall (Chapter 2), supports this theory even further, since it shows that these wavelets can perpetuate themselves for long periods of time. Therefore, it seems unlikely that these multiple wavelets are daughter waves of a macro-reentrant mother waves. located elsewhere, or that they are derived from a rapidly firing focus, ${ }^{139-4 m, 3}$ and thus play no role in perpetuating AF. There might be a macro-reentrant circuit or focus present, but in these cases there is no need for another explanation. However, we observed focal activation in a small percentage of beats. Wo could not exclude abnormal impulse formation, but since we performed our experiments in comparatively healthy hearts, we thought abnomal impulse formation unlikely. In our acute experiments, abnormal impulse formation does not seem to play a role. Epicardial breakthrough of impulses conducting over trabeculae was considered more feasible ${ }^{\text {th }}$ However, it seemed that during chronic atrial fibrillation focal activation was more common (Chapter 4). This might be the result of the smaller size of wavelets, which allowed them to conduct in lower layers or trabeculae. However, abnormal impulse formation can not be excluded. 


\section{Type / Fibrillation}

In cases of type a fbrllation, the perpetuating mechanism is less clear. The study of Cox al an 25 dogs and a few patients, demonstrated macrorentry as a mechanism for atrial fibrillation. It seems possible that in our type 1 patients (Chapter 2), the broad single wavefronts in the right atrium are part of such a large mother wave, or that they are passive daughter waves. The rare observation of reentry within the scalle of the mapping area, also indicates that reentry in these patients occurs on a larger scale or elsewhere. However, in the goat single wavelets dominated during acute atrial fibrillation and were virtually absent during chronic atrial fibrillation (Chapter 4). Does this indicate that the propagating mechanism is completely different? The gradual increase in the duration of atrial fibrillation unt it becomes chronic, ${ }^{\text {sto }}$ suggests that there is also a gradual shift in the mechanism. Apparently, the difference between perpetuation by multiple wavelets or by a macro-reentrant mother wave hes in the scale in which reentry occurs. This option is also in accordance with the observation that the degree of complexity of activation in the patients demonstrated gradual differences instead of a clear cut-oft point between the different types (Chapter 2). The type of fibrillation, and thus the number of wavelets, that is simultaneously present is then indicative of the scale of reentry.

\section{Number of Wavelets}

It has been estimated that a minimal number of four to six wavelets should be simultaneously present in the whole atria to maintain fibrillation with multiple wavelets. In the chronically instrumented goat we estimated the fibrillatory pathlength by multiplying the median interval by the median conduction velocity (Chapter 4). The pathlength during chronic AF (average $10 \mathrm{~cm}$ ) was 2.3 times smaller than in acute fibrillation. This indicates that when no dilatation has occurred, about five times as many wavelets will fit into the atrial tissue. In case of atrial ditation, even more wavelets could be present. "This explains a greater stability of chronic fibrillation, since the chance that all wavelets die out simultaneously is acoondingly diminished. If we applied the same calculation to the patients with alectrically induced atrial fibrillation, the estimated pathength would be in the order of $11 \mathrm{~cm}$ in type $1 \mathrm{AF}$, and $8 \mathrm{~cm}$ and $5 \mathrm{~cm}$ in the patients with type II and type 1 a fibrillation, respectively (Chapter 2). This indicates that the number of reentrant wavelets in the mapping area of $3.6 \mathrm{~cm}$ diameter (surface area $10.2 \mathrm{~cm}^{2}$ ) would be about 1,2 and 5 respectively. The surface of the human 
atria was estimated at about $60 \mathrm{~cm}^{2}$ in humans, ${ }^{160}$ and the maximal number of wavelets in the whole of the atria might therefore be estimated at 6,12 , and 30 respectively. This number of wavelets in type nif fibrillation might even allow for three dimensional conduction, making it as stable as ventricular fibrillation. However, when the wavelets are wide or spread centrifugally, the actual number might be significantly smaller. Therefore, a single mother wave with a small cone is still one of the possibilities. Nonetheless, it seems probable that even in the patients with type I fibrillation more than one wavelet perpetuates As. The largest estimated pathlength in our patients was $15 \mathrm{~cm}$, resulting in a estimate of three reentrant wavelets that are capable of sustaining atrial fibrillation.

It remains possible that in some patients a single circuit might truly be the source of the induced fibrillation. The irregular atrial rate would then originate from a varying revolution time of a mother wave, or the presence of irregularly conducting daughter waves. ${ }^{58}$ In such cases the difference between fibrillation and an irregular futter would be small. Actually, the fibrillation rate in our type i patients was similar to rates that nomally are found in atrial futter. In type $1 \mathrm{AE}$ the average atrial rate was 345 bpm, which is near the upper limit of type 1 atrial flutter rates $(240-340 \mathrm{bpm})$, and in the lower limit of type 11 flutter $(340-433$ bom). A difference between thutter and fibrillation then lies in the variability in beat-to-beat atrial cycle length. This did not differ between type and type u flutter, and ranged between $1-17$ milliseconds. ${ }^{\text {s. }}$ our patients with atrial. fibrillation exhibited a larger variability, but in type 1 fibrillation the variation of interval was significantly smaller than in other patients $(54$ milliseconds versus. 94 and 104 milliseconds). It seems logical that the variations of cycle length that are caused by conduction disturbances within a dominant circuit are fairly small in comparison with the variations that are due to multiple reentraint wavelets. The amount of cycle length variability in type fibrillation seems 100 large to be attributable to a single circuit, but might be more consistent with a comparatively small number of circuits.

Most goats and patients also had beat-to-beat variations in the degree of complexity of activation and showed several beats of another type than their dominant type of fibrillation (Chapters 2 and 4 ). This indicates that the degree of complexity of activation during $A F$ is not stable in time, independent of the stability of the arrhythmia. It seems to be normal feature of the mechanism that it passes through the whole spectrum of complexity of activation in most individuals. The notion that the scale of reentry varies during atrial fibrillation is applicable to 
these temporal variations; since it is unlikely that the number of wavelets that die out and are generated is similar at all times, it is likely that the number of reentrant wavelets will vary. The apparently random movement of the wavelets in itself causes variations in the degree of complexity, and variations of the scale of rentery.

\section{A Decreasing Scale of Reentry: from Atrial Flutter to Chronic Atrial Fibrillation?}

Possibly atrial flutter and atrial fibrillation are part of a continuous spectrum of reentrant arrhythmias. A regular single circuit results in type I atrial hutter, irregularities cause type in flutter, and major irregularities in a single circuin (mother wave with daughter waves) or the simultaneous presence of a few relatively large reentrant circuits might induce type fibrillation. Then a gradual decrease of the scale of reentry, and a consequent increase in the number of wavelets, could account for type 11 and type $n$ AF, and could eventually cause AF to become chronic. It would be interesting to investigate the pathlength of wavelets in the beats just priou to termination of atrial fibrillation. It is likely that there is a critical pathlength for perpetuation of atrial fibrillation. Since the different types of fibrilation in humans were all equally stable, it is not the awerage pathlength that determines whether af perpetuates. Instead, there seems to be a maximal pathlength for $A F$. In other words, when the pathlength exceds a certain value, the chance for termination becomes $100 \%$. In a similar way the chance for intertuption might become $0 \%$, when the pathlength drops below a certain value. Possibly, the critical pathlength for chronic fibrillation is that which allows for three dimensional propagation of wavelets. This is in accordance with the observed difference between acute and chronic atrial fibrillation (Chapter 4).

A concept of similar mechanisms for atrial flutter and atrial fibrillation was also described by Cox et al. ${ }^{\text {sw }}$ Their measurements were performed in waw-patients with more or less similar characteristics to the ones studied by us, and in a carine a model of "pathological atrial fibrillation" due to chronic mitral regurgitation. It differs from the suggestion above in the sense that not the scale of reentry, but the differences in the degree of irregularity of both a dominant circuit, passive daughter waves and the atrio-ventricular conduction were suggested to determine the diagnosis of different atrial arthythmias. The mechanism of atrial flutter and fibrillation was thought to be single circuit reentry. However, an exception was made for some cases of atrial hibrillation in which no large circuit could be found. and multiple wavelets were assumed. However, the mapping system used by them 
had an interelectrode distance that was approximately the times larger than in our measurements, and therefore micro-reentry would have been difficult to wisualize. Cox et al. did not account for differences between acute and chronic atrial fibrillation. The duration of episodes was not mentioned, but it seems that atrial fibrillation was self-terminating rather than chronic, since in all individuals atrial fibrillation was induced by burst pacing. Cox"s and our concept of atrial fibrillation might be in agreement with each other, assuming that in the cases in which Cox could not determine a large reentrant circuit, teentry took place on a smaller scalle.

However, many aspects remain unclear about the mechanism of atrial fibrillation. If the difference between the different types of atrial fibrillation and atrial nulter is the scale of reentry, what then is the role of anatomical obstacles? Historically, a clear division has been made between the mechanism of reentry around an anatomical obstacle and the physiological "leading circle reentry". "ln the former, the size of the obstacle determines the time the wavefront ravels until it returns to its starting point. The circulation time is longer than the refratory period and therefore an excitable gap is present. This allows the wavefront to reenter into fully excitable tissue, and explains the stability of anatomical reentry. Leading circle reentry is dependent on a short refractory period and for slow conduction in order to be able to reenter, and originally it was thought that no excitable gap was present. " However, later a short excitable gap was demonstrated during physiological reentry. ${ }^{61,7}$ Thus, the question arises whether the implementation of anatomical obstacles changes the mechanism? And does it create an essential difference between flutter and fibrillation? On this matter the data are inconsistent; although atrial flutter is generally regarded as an arrhythmia that arises from reentry around an anatomical obstacle, $36,82,185,100.205$ it has been shown that such an obstacle is not a prerequisite. "sis the study of Cox et al. demonstrates that in some cases of atrial fibrilation the pulmonary and/or caval veins were involved in the apparent reentrant circuits, while in other cases reentry was purely functional. ${ }^{58}$ Possibly the implementation of an anatomical obstacle within a reentrant circuht, does not cause the difference between fluter and fibrillation, but is the logical consequence of a larger sized circuit.

Another observation that is difficult to explain, is that it is a faily common finding

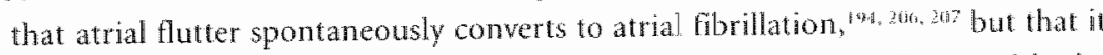
seems rate the other way around. ${ }^{2}$. The former is in acondance with the mechanism of atrial remodeling that occurs during continuous rapid atrial 
activation, and fawors atrial fibrillation. ${ }^{130,354,160,20 \% 21 !}$ But the latter is more difficult to understand. Why does atrial fibrillation rarely convert to atrial flutter when it terminates spontaneously? 2 "The idea that a specinc anatomical substrate is needed for atrial flatter might be the explanation in most cases, but as has been discussed previously, such an anatomical substrate is not a prerequisite. Possibly, the chance of atrial flutter arising without an anatomical obstacle is low.

Other factors than the pathlength, which is a product of the median interwal and the median conduction velocity, must also play a role in the mechanism of atrial fibrillation. The study of Wiffels et. al. "3h showed that the major decrease of intervals occurred within the first 24 hours of AF induction while still only short runs of Af could be induced. Chronic af did not occur until much later. In chapter 4 it was demonstrated that even during chronic atrial fibrillation the conduction velocity remains normal for the atrial rate. Thus the pathlength is almost as short after 24 hours as it is during chronic Af, but after 24 hours atrial fibrillation still stops. Not until an average of eight days of continuous induction has passed does AF become chronic. Wijffels et al. ${ }^{130,150}$ suggested that increased heterogeneity of conduction and excitability are of crucial importance for this additional effect. Development of atrial dilatation was thought to be involved, but acute overfilling of the cardiovascular system with 0.5 to I liter showed no effect on the atrial refractory period. However, it remained unclear whether the atria of the goats in chronic fibrillation were larger than before. 150 No effect was found from ischemia, $A N F_{3}$ increase in (para)sympathetic activity and a higher sensitivity to neurotransmitters. ${ }^{130, \text { sis }}$ Aso, structural conduction disturbances did not seem to be involved in the difference between acute and chronic af (Chapter 4 ).

Thus, differences in the degree of complexity of atrial fibrillation might be explained by differences in the scale of reentry, but the difference in duration of selftermunating acute $A$ f and chronic atrial fibrillation, and the difference between atrial fibrillation and flutter is probably dependent upon more factors. There may still be a sole for a critical number of reentrant wavelets, but then the total surface must have increased to a critical value by atrial diatation. Enhancement of the nonuniform anisotropy and local depression of action potentials might develop gradually and causes increased heterogeneity of conduction. ${ }^{2 \%, 23,25}$ Together with an increased spatial dispersion of refractory periods, ${ }^{210-219}$ this would increase the chance for reentry. ${ }^{2}$. ${ }^{2}$ Hereby the same number of wavelets might obtain a greater chance of perpetuation and thus ar might become chronic. However, the study of wijhtels et al. demonstrated a decrease of spatial dispersion 
of refractory periods within 24 hours of maintenance of $A=$, and this remained low after a fortnight. ${ }^{150}$ It is undear whether the natural nonuniform anisotropy in the atria was augmented by continuous induction of atrial fibrillation. Moreovet depression of action potentials was not measured. At this point many factors of the mechanism of the physiological tendency of atrial fibrillation to become chronic remain an enigna.

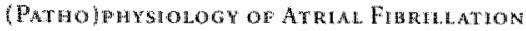

\section{Physiological Atrial Fibrillation}

\section{Acute Atrial Fibrillation}

It is important to realize that the atrial fibrillation investigated in this thesis is of a physiologicall nature rather than a pathological. Most of the patients had no spontaneous episodes of AF documented and none suffered from chronic atrial fibrillation. Cardiac abnormalities other than the accessory pathway were ruled out. Their attria showed a normal spread of activation during sinus rhythm and rapid atrial pacing. Even during atrial fibrillation the conduction velocities of type I beats were normal to the atrial rate (Chapter 2). Athough it is unknown to what extent the existence of an accessory pathway affects the characteristics of the process of atrial fibrillation, the atria of these patients were apparently normal. The goats were normal healthy individuals, and also in their hearts short runs of atrial fibrillation could be readily induced by burst pacing (Chapter 4). Induction of atrial fibrillation by pacing is not physiological, but the mechanism of perpetuation seems to be intrinsic to the normal heart. *

\section{Chronic Atrial Fibrillation}

It is difficult to picture chronic atral fibrillation as a physiological phenomenon, and obviously artificial maintenance of atrial fibrillation is unmatural. But Gordon $\mathrm{K}$ Moe demonstrated that $A$ is an arrhythmia that is independent of its progenitor" (Chapter 1), and the atrial adaptation that results in chronic atrial. fibrillation might be a physiological mechanism as well. In contrast to other animal studies, which induced atrial fibrillation with artificially obtained mitral regurgitation ${ }^{\text {s. }}$ or sterile pericarditis, ${ }^{20}$ the goats that we studied had been in normal sinus thythm all their lives and had no pathological substrate for atrial fibrillation. By artifial maintenance of atrat fibrillation, the duration of spontaneously terminating episodes increased progressively tuntil finally chronic 
atrial fibrillation developed. 3 . A previous study of Wijffels et al. in the same goat model, suggested that the physiological adaptation of the fibrillatory rate plays an important role in this domestication of atrial fibrillation. "The cascade of cause and effect, finally leading to chronic atrial fibrillation, is started by a shortening of the fibrillation interval. As soon as the fibrillation interval passes a critical threshold of 1.20 milliseconds, obviously atrial fibrillation becomes more stable and the duration of AF starts to increase. This in turn will further shorten the fibrillation interval which will prolong the duration of AF again, etc. Such a positive feedback mechanism will continue until a new steady state is reached in which atrial fibrillation has become the predominant atrial rhythm." 230 "There are additional arguments for the concept of physiological chronic atrial fibrillation. Atrial conduction velocity during regular rates was similar before and after induction of chronic atrial fibrillation, and remained normal for the atrial rate during chronic AF. Although chronic implantation of electrodes might cause scarring and therefore might form a substrate for chronic AF, several findings suggested that in our experiments this was of minor importance. Areas of structural conduction block were absent during chronic AF within the boundaries of the right atrial free wall (Chapter 4). Also, the study of Wijffels et al. demonstrated that the changes imposed on the atria by fibrillation were reversed one week after restoration of sinus rhythm. ${ }^{130}$ Thus, it seems that the adaptation of the atria to fibrillation is functional rather than structural, and is intrinsic to the normal heart.

The concept of physiological atrial fibrillation emphasizes the difference between the artificially induced AF that we studied, and the spontaneous AF that occurs in patients with more or less underlying heart disease. Possibly there are different mechanisms involved.

\section{Pathological Atrial Fibrillation}

In most patients, AF is not the only cardiac malfuction they suffer from. Atrial fibrillation is strongly associated with increasing age, 221,222 coronary heart disease, ${ }^{2: 3}$ hypertension, valvular disease, cardiac failure or rheumatic heart disease. ${ }^{221}$ Also several factors are known to promote the persistence of AF. These include increased atrial mass, atrial dilatation, acute and chronic myocardial injury, fibrosis of the sino-atrial node and increased vagal tone. 22.225 


\section{Mechanism: Multiple Wavelets?}

It is commonly believed that also in pathological Af multiple wavelets are the perpetuating mechanism for atrial fibrillation. 23 . The possible role of multiple wavelets in pathological atrial fibrillation is even more likely when there are structural abnormalities in the heart. Age, injury and fibrosis are related to increased amounts of non conductive tissue in the atria, ${ }^{2 y}$ and therefore facilitate division of a wavefront into independent wavelets and cause zigzag conduction that results in short apparent wavelength $s^{73}$. Atrial dilatation, ${ }^{22}$ ischemia, hypertension, cardiac failure, and increased vagal tone, all induce dispersion of refractory periods and/or conduction velocities ${ }^{22 k}$ and thereby increase the likelihood of development of multiple wavelets. Larger atria can harbor even more of these randomly reentering wavelets. It seems that in patients pathophysiological changes due to aging or underlying cardiac disease will be unevenly distributed through the atrial tissue. By assessment of the distribution of abnormal electrograms, areas that consistently demonstrate conduction disturbances can be identified (Chapter 3). Selective ablation of such regions with structural conduction disturbances might cure Ar: The fact that the results of the Maze operation for atrial fibrillation 229 are better than those of the corridor operation ${ }^{230-232}$ and the left atrial isolation procedure, ${ }^{23.3}$ also indicates that the scale of reentry is smaller than the dimension of one atrium.

\section{Mechanism: Mother Waves?}

Areas of scar tissue can also function as an anatomical obstacle around which a mother wave rotates. Single circuits during AF in diseased atria have been demonstrated by $\mathrm{COX},{ }^{58}$ and during chronic AF single meandering circuits have been recorded incidentally. ${ }^{179,202,234}$ Furthermore, it has been suggested recently that multiple wavelets are a transient phenomenon underlying only carly stages of Af. After this the multiple wavelets are thought to organize into an single meandering circuit for AF to sustain. Moreover it has been found, that interruption of the circuit by burst pacing succeded to terminate af in a few cases. $^{17 \%}$

\section{Mechanism: Foci?}

In diseased atria the likelihood for abnormal impulse formation may be increased, particularly in ischemic disease and atrial stretch. ${ }^{23}$ Calcium overload could cause delayed afterdepolarizations-induced triggered activity, ${ }^{2.36}$ and it was 
discussed that atrial fbrilation itself might cause such calcium overload. 150 , ito Focal origins of $\mathrm{AF}$ have recenty been indicated by several groups. $180-383.236$ They do not prove that such foci are triggered activity or abnormal automaticity but dearly show that in some patients A F initiates on the basis of a highy localized mechanism. When the focus has a fixed location, ablation might cure the patient from Af. Patients with an arthythmia of focal origin may occasionally be readily identified. The patients in the studies of Jais ${ }^{181}$ and Haissaguerrev? all suffered from paroxysmal atrial fibrillation and irregular atrial tachycardia or monomorphic extrasystoles. During atrial arrhythmia a focal source of activation was confirmed in all nine patients by a consistent and centrifugal pattern of activation as recorded from conventional ablation catheters or multi-electrode catheters. ${ }^{237}$ However, such mapping was performed during a in only two patients. Foci were found near the sinus node, in the ostium of the coronary sinus, and at the ostia or junctions of the pulmonary weins. The site with earliest activity was ablated, which proved to be successful in every patient (one patient needed two procedures). During a average follow-up of 10 two months, neither AF nor atria! tachycardia returned. In diseased atria, triggered or automatic foci may play a role during atrial fibrillation. If such foci have a variable rate or a variable number, the activation pattern might be variable also, and might appear as any type of fibrillation.

\section{Physiological Adaptation}

In any case of atrial fibrillation, whether it occurs in a normal or in a diseased heart, the arrhythmia itself seems to have a propensity to induce changes that favor progression. ${ }^{25} .221 .238$ In the chronically instrumented goat, it was shown that maintenance of 24 hours of atrial fibrillation reversed the physiological rate adaptation of the refractory period. Actually the refractory period during sinus rhythm became shorter than during fast rates. ${ }^{1 \text { in }}$ This suggests that a patient with a stable, but diseased condition of the heart, who suffers from a single episocle of atrial fibrillation that lasts for a day, has an increased risk for spontaneous reinduction after sinus rhythm has been restored. The cascade leading to chronic atrial librillation might be initiated then, while the original mechanism that caused the first episode of atrial fibrillation is not necessarily involved. Treatment of A in early stages seems to be necessary in order to prevent deterioration in to chronic As. 


\section{Current Situation}

Historically, atrial fibrillation has been regarded as a minor health problem. The majority of patients is of advanced age and often tolerates the arrhythmia quite well. ${ }^{2.3}$ Therefore, treatment has generally been limited to ventricular rate control with digitalis. However, atrial fibrillation is a cause of substantial morbidity, ${ }^{2: 4}$ and a mortality that is twice as high as in control subjects." When atrial fibrillation is left untreated it tends to become chronic spontaneously ${ }^{10}$ and causes cardiomyopathic damage. ${ }^{242}$ Moreover up to $30 \%$ suffer from thromboembolic events. ${ }^{2.3-2.25}$ Up to $20 \%$ of humans have experienced $\mathrm{AF}$ by age of $80,{ }^{246}$ of which one-third become chromic patients. ${ }^{78,2}$. 3 ? The present antifibrillatory drug strategies only successfully maintain sinus rhythm in about $50 \%$ of the patients, and are still severely hampered by systemic side effects, and pro-arrhythmia. ${ }^{241}$ Several studies even suggest that the use of antiarrhythmic drugs may be associated with increased mortality. ${ }^{241,248}$ Every anti-arrhythmic drug is capable of producing a pro-arrhythmic response and this risk is attenuated in the typical atrial fibrillation patient, in whom often multiple cardioactive agents are combined and underlying cardiovascular or systemic disease is present. Obviously the risk is also higher during chronic treatment than during acute intervention. ${ }^{249}$ Therefore, there are clear indications for treatment. However, after 150 years $^{2}$ of research it sometimes seems that only little improvement has been achieved.

The ideal treatment of atrial fibrillation would restore and maintain sinus rhythm without any pro-arrhythmic side effects, since it optimizes cardiac performance and protects against thrombo-embolism. Also, the effect of atrial remodeling, and thereby the progression of paroxysmal to chronic ar would be abolished. Recent studies have shown that the pro-arhythmic effect of $\mathrm{Al}$ in itself is reversed by restoration of sinus rhythm. ${ }^{30}$ Therefore, pharmacological or electrical cardioversion seems to be a reasonable strategy.24.249 Once sinus rhythm is restored, many patients revert AF rapidly if they are not further treated. Maintenance of sinus rhythm is even more difficult than conversion of AF. If sinus rlyythm cannot be obtained, ventricular rate should be controlled, and thrombo-embolism should be prevented. Rate control is no cure for atrial fibrillation and does not improve the thrombo-embolic risk, nor does it fully restore the hemodynamical function of the heart. The importance of rate control 
in current practice illustrates the problems that are presently encountered in the treatment of atrial fibrillation. Relief of symptoms is often the only remaining solution. Treatment of AF in patients with severe underlying disease should be aimed at treatment of that disease itself. Solitary treatment of AF in such cases is severely hampered by side effects, drug interactions and scarcity of data.

\section{Recent Developments}

\section{Excitable Gap}

To stop atrial fibrillation a method has to be sought that minimizes the number of wavelets, or makes it impossible for new wavelets to generate and reenter. Antifibrillatory drugs are thought to achieve their effect by prolongation of the atrial wavelength, thereby diminishing the number of wavelets. ${ }^{59,165,484,250-252}$ However, it has recently been shown that prolongation of the wavelength during atrial fibrillation does not occurr during successful pharmacological conversion of chronic atrial fibrillation in the goat. Instead it was observed that all tested types of drugs progressively widen the excitable gap. ${ }^{148}$

Increase of the excitable gap might add to successful pace termination of AF, which has been first suggested and tested in chronically instrumented dogs by Allessie and Kirchhof. ${ }^{617} 7$ The large variability of intervals was thought to indicate that the median interval is longer than the atrial refractory period. Allessie and Kirchhof believed it possible that the long intervals are shortened by pacing. Capture over an area with a diameter larger than two $\mathrm{cm}$ was achieved, but termination of $\mathrm{AP}$ was not obtained. Experiments in a small number of patients with chronic $\mathrm{AF}(\mathrm{n}=3),{ }^{253}$ demonstrated regional entrainment in the right atrium over a radius of at least 2.8 $\mathrm{cm}$ and a cycle length rage of 13 milliseconds or more. However, termination of $\mathrm{AF}$ was not obtained. Interestingly, in one patient defibrillation with low energy was possible during entrainment, whereas higher energy shock had failed before entrainment. ${ }^{159} 253$ Another study obtained capture during type I fibrillation in humans $(n=24){ }^{254}$ Possibly, different types of AF offer different opportunities. Whether improvement of the pacing mode (adaptive entrainment) or a combination with antifibrillatory drugs would allow pace termination remains speculative. So far, multi-site high frequency pacing has proven not to be effective. ${ }^{295}$

The notion that sharp $U$-turns at pivoting points can induce an excitable gap and fawor reentry, might also allow for treatment. Drugs that depress the upstroke of the action potential (Class I) or increase the amount of current needed to be excited, might decrease wavefront curvature. Total block of u-turn would 
presumably be most effective, but is not a prerequisite. Even a small degree of depression of wavefront curvature might be effective; if the amount of excitatory current of the small number of cells at the pivoting point forces the wavefront to postpone its turn, the circuit size increases, thus allowing fewer wavelets to be present at the same time.

\section{Alternative Approaches}

At this point it is unclear whether patients may benefit from treatment of their: indiwidual type of fibrillation. Therefore, the relevance of the different mechanisms. has to be studied. In cases of macro reentrant atrial fibrillation, inter ruption of the circuit should stop the arrhythmia. Possibly treatment analogous with atrial flutter might be successful. Rapid and irregular focal activation of the atria, causing an $\mathrm{ECO}$ to exhibit the characteristics of atrial fibrillation, might be treated as a focal phenomenon. Possibly selective ablation might cure the patient. ${ }^{181 .}{ }^{182}$ It has to be studied, however, whether cases of "focal AF" have an anatomically fixed origin.

Other ablative and surgical procedures that reduce the possibility for wavelets to reenter, are recently becoming popular. The first types of such procedures, either isolated the laft atrium, ${ }^{233}$ or created a corridor through which the sinus node impulse was conducted to the atrioventricular node. 230 Thereby, the chance for sinus rhythm was increased, but a large part of the atria was withdrawn from atrial transport function. Cox et al. 20 then developed a procedure in which a "maze' of scars was created in the atria, which allowed for conduction to most parts of the tissue, but made it unlikely that wavelets could reenter. At present, several variations and ablative versions of this "maze' operation are being investigated. $156,181,256-25 \%$ However, these procedures are fairly radical. Possibly, it suffices to ablate only regions with structural conduction disturbances. Then assessment of the distribution of abnormal electrograms might be useful.

Prolonged duration of an episode and rapid reinduction of Af favor the development of chronic atrial fibrillation. ${ }^{130}$ This effect might be addressed by a system that automatically delivers drugs, but only during atrial hibrillation, immediately after the start of an episode. An apparatus is being developed that sensing the onset of atrial fibrillation, releases a fibrillation converting drug from an internal reservoir unto the atrial cavities or cononary arteries. ${ }^{25 \%} 260 \%$ However, it is to premature too draw definite conclusions.

Further research should be aimed at the electrical activation patterns of patients with spontaneous atrial fibrillation, both in the acute and chronic state. 



\section{Appendix | Summary |}

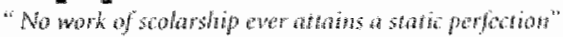

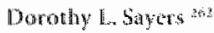




\section{Summary}

The present thesis describes the epicardial activation patterns during electrically induced atrial fibrillation in humans and goats, in an attempt to elucidate the mechanism of this fairly common arrhythmia. In chapter 1 the history of studies on the mechanism of atrial fibrillation is given. The first description of atrial fibrillation was published in 1887 . At that time it was thought to be caused by a socalled "delirium of the heart". In 1909-1910 the first uniform criteria for the diagnosis of atrial fibrillation were formulated: total irregularity of the ventricles, absence of organized atrial activation, and irregular fast oscillations of the baseline ECG Sir Thomas Lewis demonstrated in the period of 1910-1925, that disturbances of the electrical excitatory current in the atria cause atrial fibrillation. He concluded that the electrical impulse follows an irregular circular track, constantly reentering its own tail. The striking irregularity of atrial fibrillation was explained by the high rate, causing the circulating impulse and its offsprings to travel through partially refractory tissue, thus creating a sinuous course. Gordon K Moe showed in the period of 1959-1968, that atrial fibrillation $(\mathrm{AF})$ is independent of its origin, and hypothesized that an irregular impulse becomes fractionated when it divides at areas of refractory tissue, even in normal atria. The resulting offsprings would be independent of the original wavefront. "Fully developed atrial fibrillation would then be a state in which many such randomly wandering wavelets coexist." As long as the local refractory periods are sufficiently short and heterogeneous, any premature impulse can generate multiple wavelets. Their path is then determined by functional obstacles, and parameters that influence perpetuation of AF would be the mass of the atria, refractory period, conduction velocity, and excitability of the local atrial, tissue. Experimental proof of Moe's multiple wavelet theory was found in dog hearts (1985). Mulitiple wavelets wandering around natural anatomical obstacles and functional arcs of conduction block were shown. It was concluded that the critical number of wavelets for perpetuation of atrial fibrillation is between three and six. However, mapping of the excitation during atrial fibrillation in humans was still limited to a small number of patients and no systematic analysis had been done.

In chapter 2 , the electrical activation pattern during electrically induced atrial fibrillation in a group of 25 relatively young wPw-patients is described. Atrial activation is mapped using a spoon-shaped electrode containing 244 unipolar electrodes. The free wall of the right atrium is activated by more or less fragmented wavefronts. However, large individual differences in the degree of 
complexity of the activation patterns are observed, and this leads to the classification of three types of atrial fibrillation. In type 1 ( $40 \%$ of the patients). only a single wavefront conducting homogeneously, is present in the right atrium during the majority of beats, whereas in type nu $(28 \%)$, activation of the right atrium is highly fragnented and shows at least three different wavelets most of the time. Type $\mathrm{Ir}(32 \%)$ is the intermediate; with one or two inhomogeneously conducting wavelets. From type I to type III, the frequency and irregularity of the atrial rate increases, and the incidence of continuous electrical activity and reentry become higher, whereas the average conduction velocity decreases. However, no differences are found in the conducting properties of the atrial myocardium. These various types of AF in humans appear to be characterized by different numbers and dimensions of intra-atrial reentrant circuits. The possibility of these types representing different mechanisms is discussed. It is concluded that all types of fibrillation were consistent with the multiple wavelet theory, but that in type I fibrillation, a single macrorreentrant circuit may be the driving source of the arrhythmia. The suggestion that automatic foci may play a role in the perpetuation of Af cannot be confirmed. The finding of different types of atrial fibrillation offers a possible explanation for the mechanism behind the clinically observed differences between futter-fibrillation, coarse and fine fibrillation.

In chapter 3 the morphologies of the unipolar electrogram potentials reconded during the previous study are classified in order to correlate the Af electrogram configuration with the varying patterns of activation. A distinction is made between: single, short-double, long-double, and fragmented potentials. The incidence of the more complex potentials (long-double and fragmented potentials) is found to increase with the type of atrial fibrillation. Electrogram morphology is shown to be highly predictive for the occurrence of various typical patterns of conduction: rapid uniform conduction is associated with the recording of single potentials (positive predictive value [erv] of 0.96 ); around sites of collision, short-double potentials are found ( $\mathrm{pPV}$ of 0.33 ); at arcs of conduction block, long-double potentials are seen ( ppv of 0.84 ): and pivoting points or areas of slow conduction are identified by fragmented potentials ( $P$ p $v$ of 0.87). During electrically induced and self-terminating episodes of AF, no preferential anatomic sites for double or fragmented potentials are found in the right atrium. It is concluded that the morphology of unipolar electrograms during af reflects the occurrence of various specific patterns of conduction. This might be used to differentiate between different types of af and to identify 
regions with structural conduction disturbances involved in perpetuation of chronic af.

Other studies have shown that atrial fibrillation is a progressive disease. Patients with paroxysms of Af tend to become chronic sufferers. The atrial rate during the arrhythmia has been demonstrated to become faster. The question then arises whether the activation patterns during acute fibrillation are different from chronic AF. Therefore, in chapter 4 the activation patterns during electrically induced acute atrial fibrillation are compared to those recorded during chronic atrial fibrillation in seven goats. These activation patterns are recorded twice during an open chest operation, first when pacing-and recording-electrodes are implanted, and second (after $7 \pm 4$ months) when the animals are sacrificed. During both operations epicardial mapping (244 recording points) of the right atrial free wall is performed. In the acute experiments, Af is induced by burst pacing. Chronic AF (episode $>24 \mathrm{hrs}$ ) is induced in approximately one month by continuous reinduction of AF within seconds after restoration of sinus rhythm. Activation patterns during electrically induced atrial fibrillation in goats are highly comparable to those in human subjects. In comparison with the shortlasting episodes that are induced during the acute experiments, the fibrillatory rate is increased during chronic $\mathrm{AF}$. Also, the activation pattern becomes more complex (from type a to type m AF), and the number of complex potentials (longdoubles and fragmented complexes) increases accordingly. The shortest measured intervals, approximating the refractory period, decrease. Differences in conduction welocities between acute and chronic atrial fibrillation seem to be caused by a "physiological" increase in local conduction disturbances and normal rate adaptation. Thus, the conduction properties of the atria seem to remain normal. Howewer, atrial dilatation may cause an overestimate of conduction velocity, and thus, atrial conduction during chronic atrial fibrillation may actually be depressed. The estimated reentry pathlength decreases from $10 \mathrm{~cm}$ during acute $\mathrm{AF}$, to $4 \mathrm{~cm}$ during chronic $\mathrm{AF}$. It is concluded that the increasing stability of atrial fibrillation is in great part explained by a larger number of wavelets, thereby diminishing the chance of simultaneous arrest of all wavelets. The increase in the number of wavelets is attributed to a smaller circuit size (pathlength). Other factors that might play an additional role are discussed.

In the last chapter of this thesis (chapter 5), the implications for the mechanism of atrial fibrillation are discussed. The possible role of three different mechanisms that have been postulated in the literature (multiple wavelets, a single revolwing 
mother wave, and automatic or triggered foci), is reviewed. It is concluded that the results of the experiments performed in this thesis are compatible with the multiple wavelet theory, particularly during types in and in fibrillation where multiple wavelets are visible in the activation maps. The possibility is acknowledged that during type 1 af, a single mother wave might perpetuate the arrhythmia. It is suggested that the mechanism of atrial fibrillation consists of a wide spectrum of reentrant circuits of wariable size. Going from atrial flutter, via paroxysms of fibrillation, to chronic AF, the dimensions of reentry might gradually decrease, in association with an increasing number of wavelets. The data presented in this thesis cannot substantiate a role for a focal mechanism, but it is implied that spontaneous AF, in patients with diseased atria, might have a focal origin. Finally, some options for the treatment of atrial fibrillation are discussed.

In Conclusion, during electrically induced atrial fibrillation in a group of young humans, different degrees of complexity of atrial activation are found. The degree of complexity is described by classification of different types of fibrillation. Going from type I to type in, the atrial rate becomes faster and more irregular" the incidence of reentry increases, and a larger number of complex potentials is recorded. The configuration of local unipolar electrograms is predictive of local conduction disturbances. Comparison of electrically induced acute and chronic atrial fibrillation in goats demonstrates that the atrial rate increases and that atrial activation becomes more complex. Conduction remains normal for the fibrillatory rate. However, atrial dilatation might cause an overestimation of the conduction velocity. The pathlength of reentrant circuits is decreased during chronic AF. A decrease of the circuit size allows a larger number of wavelets to be present in the atria simultaneously, thereby limiting the chance for arrest of all circulating impulses and cessation of the arrhythmia. These findings may provide further insight in the mechanism of atrial fibrillation. Varying dimensions of reentrant circuits may explain the clinically observed differences between Mutterfibrillation, and coarse and fine fibrillation. However, it should not be forgotten that patients often have an underlying disease that gives rise to their $\mathrm{m}$. Thus, the clinical role of different degrees of complexily is unclear at present and further studies are needed. 

Appendix | Samenvatting | 


\section{Samenvatting}

Het hart bestat wh een sper met vier holtes; de rechter en linker boezems, en de rechter en linker kamers. De kamers (ventrikel) dienen voor het rondpompen wam bloed, naar de longslagader (rechts) en de grote lichaamsslagader (aorta, links). De boezens (atria) ontwangen bloed uit de grote lichamsaders (venae cava, rechts) en de longaders (links), en pompen dit door naar de kamers. Zo ontstaat een circulerende stroom warin zuurstof-arm bloed unt het lichaam - wa de rechter boezem en kamer - naar de longen word gepompt. In de longen wordt het bloed opnieuw wan zuurstof woorzien waarnat het - via de linker boezem en kamer - weer het lichaam wordt ingepompt, zodat de organen en weefsels van zuurstof worden woorzien.

Het hart pompt doordat een electrische impuls door de spiercellen wordt geleid. Iedere afzonderlijke cel wordt door deze impuls angezet tot samentrekken (contractie). De electische stroom die het hart aanzet tot kloppen, ontstat in de zogenamame sinusknoop. Dit is een gespecialiseerde groep cellen in de rechter boezem, wak naast de bovenste lichaamsader. Van hieruit wordt de stroom voortgeleidt over beide boezems naar de atrio-ventriculaire knoop. Dit is een groep cellen die normaal de enige electrische verbinding wormt tussen cle boezems en de kamers. De stroom activeert daarna de kamers. Gewoonlijk wordt het hart circa zo keer per minuut zo geactiveerd, en de tijd tussen twee slagen is steeds ongeveer hetzelfde. Dit nomale hatritme wordt ook wel sinusritme genoemd omdat de impuls in de simusknoop ontstaat.

Dit proefschrift gaat over boezemfibrilleren (atrium fibrilleren, afgekort AF); ecn ritmestoomis war bij de oorzaak in de boezems ligt. Fibrilleren is een zeer snel en total onregelmatig ritme (400-600 slagen per minut). Tijdens boezemfibrilleren loopt de electrische stroom niet meer wanuit de sinusknoop nat de kamers, mar zijn or in de boezems vele stroompjes (ook wel golfes of wavelers genoend) tegelik aanwezig, die kriskras door elkaar lopen. Deze golfjes kunnen soms terugkeren nar cellen die al eerder geactiverd werden, dit heet reentry. Soms keert sen golfje terug in thet weefsel dat het zelf al eerder geactivend had, of ontstat dan een kringstroom (closed loop reentry). Het komt ook voor dat ex golfie weofel heractiveet, dat eerst door een ander was geprikkeld frawdom reentry). Do atrio-ventriculaire knoop geleid slechts een deel van de vele golfjes naar de kamers, waardoor ook deze onregelmatig gaan kloppen, maar langzamer dan de boezens (100-160 slagen per minuut). Tijdens boezemfibrilleren is er dus geen goed georganiseerde hartactiviteit meer en de boezems en kamers kloppen te 
snel, te onregelmatig, en in een verschillend ritme.

Boezemfibrilleren komt zeer frequent voor bij de mens, en hoe ouder men word, des te vaker. Door de onsamenhangende, te snelle hartactiviteit klagen patienten over harkloppingen. Het hartritme past zich niet meer an bij lichameligke inspanning, doordat het niet meer door de sinusknoop geregeld wordt. Ondat de boezems niet meer als geheel samentrekken, maar allerlei gedeeltes aronderlijk wan elkaar contraheren, staat het bloed op sommige plaatsen vrijwel stil. Hierdoor kunnen bloedstolsels (thrombi) ontstan, en kan men stolsels in de bloedvaten wan de longen (longembolie) of hersenen (herseninfarct) krijgen. Dit worden thromboembolische complicaties genoemd.

Hoofdstuk 1 beschrift de geschiedenis van het onderzoek naar boezemfibrilleren. De eerste beschrijing van een hartritme dat we nu kennen als af werd gepublicerd in 1887. Men dacht toen dat dit veroorzaakt werd door een zogenaamd delirium van het hart. In $1909-1910$ werden de eerste eendulidige criteria voor de diagnose van boezemfibrilleren geformuleerd: totale inegulariteit van de kamers, afwezigheid van georganiseerde boezem activitet en onregelmatige, en snelle trillingen in de grondlijn van een hartilmpje (eCG). Sir Thomas Lewhs toonde in de periode van 1910-1925, dat verstoringen van de electrische prikkel in de boezems de ootzak zijn van boezemfibrilleren. Hij concludeerde, dat de electrische impuls een onregelmatige rondgaande route volgde; steeds in aijn eigen start bijt. De opvallende onregelmatigheid van boezemfibrilleren werd verklaard door het snelle ritme. Hierdoor moeten de circulerende prikkel en de ander golfyes, die ervan afstammen, door weefsel geleiden dat nog niet helemal hersteld is wan de vorige activiteit (partieel refractair). Sommige cellen zijn dan nog niet in stat om opnieuw geactiveerd te worden, terwijl andere cellen dir al wel kunnen of vertraagd reageren. Zo ontstaat een kronkelende route. Gordon $\mathrm{K}$ Moe het daama in de periode $1959-1968$ zien, dat boezemfibrilieren omalnakelijk is van de prikkel die het opwekt. Hij veronderstelde dat een onregelmatige impuls zich opsplits (fractioneert) als het refractair weefsel tegen komt, zelf in normate gezonde boezems. De hierüt voortkomende afstammelingen zouden onafhankelijk zijn wan het oorspronkelike golfe. "Volledig ontwikkeld boezemfibrilteren zou dan een toestand zijn waarin vele zulke in het wilde weg (random) zwervende golfjes naast elkaar bestaan." Zolang de plaatselijke herstel tijd (refractaire perionde) kort en heterogeen genoeg is, kan iedere vroeglijdige impuls meerdere golfjes opwekken. Hun pad word dan bepald door weefsel dat op dat moment niet geactiveerd kan worden functionele obstakels, 
geleidingsblokkade). Het voortbestaan van boezemfibrilleren zou dan afhangen van de hoeveelheid boezenweefsel, de refractaire periode, de geleidingssnelheid, en de prikkelbaarheid van het plaatselijke boezemweefsel. Moe's veelvoudige (multipele) golfjes theorie werd experimenteel bewezen in hondeharten (1985). Men liet multipele golfjes zien die rond zwierven om anatomische obstakels en functionele lijnen van geleidingsblokkade. Er werd geconcludeerd dat het minimale aantal golfjes dat nodig is om boezemfibrilleren te doen voortbestaan ergens tussen de drie en zes ligt. Maar, nog steeds was de activiteit tijdens boezemfibrilleren bij mensen nauwelijks in kaart gebracht, en er was nog geen systematische analyse uitgevoerd. De experimenten die in het kader van dit proefschrift zijn uitgevoerd, brengen de route die de golfjes tijdens boezemfibrilleren afleggen gedetailleerd in kaart (high-density mapping). Dit is van belang omdat er nog veel vraagtekens zijn over het mechanisme wan boezemfibrilleren en behandeling nog vele problemen oproept.

Hoofdstuk 2 beschrijft het electrische activatiepatroon tijdens boezemfibrilleren, dat kunstmatig is opgewekt (met woortijdige electrische prikkels), in 25 vrij jonge patiënten, die een abnormale tweede (accessoire) geleidende verbinding hadden tussen de boezems en kamers (Wolff-Parkinson-White syndroom). Deze patiënten hebben wrijwel normale boezems, maar hun neiging tot boezemfibrilleren is groter dan in gezonde mensen. De activiteit van de vrije wand van de rechter boezem werd in kaart gebracht met een lepelvormige electrode waarin 244 enkelpolige (unipolaire) electrodes zitten. De boezem blijkt steeds geactiveerd te worden door min of meer gefragmenteerde golfjes, maar de mate waarin de actiwatiepatronen ingewikkeld zijn (graad wan complexiteit), verschilt per patiënt. Dit leidde tot een onderverdeling (classificatie) van drie types boezemfibrilleren. Tijdens type I ( $40 \%$ van de patiënten), is er maar een enkel golfje, dat gelijkmatig voortgeleidt, aanwezig tijdens de meeste slagen. Terwijl tijdens type in ( $28 \%)$, de activiteit in de rechter boezem de meeste tijd sterk gefragmenteerd is, en er minstens drie verschillende golfjes tegelijk aanwezig zijn. Type nn (32\%) ligt tussen de andere twee in, met een of twee ongelijkmatig voortgeleide golfjes. Van type I naar type IIr, neemt de snelheid (frequentie) en onregelmatigheid van het boezemritme toe. Het aantal keren (incidentie), dat er voortdurende electrische activiteit en reentry optreed groeit, terwijl de gemiddelde geleidingssnelheid (conduction velocity) afneemt. Toch zijn er geen verschillen gevonden in de geleidingseigenschappen van het boezemweefsel. Deze verschillende types boezemfibrilleren in de mens lijken gekenmerkt te worden, door verschillen in het 
antal en formaat van reenterende circuits in de boezems. De mogelikheid dat deze types verschillende mechanismen van boezemfibrilleren weergeven wordt besproken. De conclusie is, dat alle types boezemfibrilleren overeenstemmen met de multipele-golfes-theorie, maar, dat in type I fibrilleren ook èn enkel groot reenterend circuit de bron van de ritmestoornis zou kunnen zijn. In de literatuur wordt gesuggereerd, dat een afwijkende groep cellen die konstant electrische impulsen afvuurt (focus), een rol zou kunnen spelen in het voortbestaan van boezenfibrilleren. Dit kan niet worden bevestigd in het huidige onderzoek.

In hoofdstuk 3 worden de vormkenmerken (morfologie) geclassificeend van de potentialen (tekens van lokale activiteit) die zichtbaar zijn in de unipolaire electrogrammen uit de vorige studie. Dit werd gedaan om verband te leggen tussen de vorm wan electrogrammen en de verschillende activatiepatronen. Er wordt onderscheid gemaakt tussen: enkelvoudige-, korte dubbelvoudige-, lange dubbelwoudige- en veelroudige- (gefragmenteerde) potentialen. De incidentie van de complexere potentialen (lange dubbele- en gefragmenteerde potentialen) blijkt toe te nemen met het type boezemfibrilleren. De electrogram morfologie is sterk voorspellend voor het optreden van verschillende typische geleidingspatronen: snelle gelijkmatige geleiding is gekoppeld an meting van enkelvoudige potentialen. Als enkelvoudige potentialen worden gezien is de kans, dat op die plats de geleiding snel en gelijkmatig zal zijn, bijna 100\% (positicf voorspellende waarde $[P P V], 0.96)$. Rond plaatsen waar twee golfjes op elkaar botsen (collision) worden korte dubbelvoudige potentialen aangetroffen (PPV, 0.33); bij lijnen van geleidingsblokkade worden lange dubbelvoudige potentialen gezien ( $\mathrm{ppv}, 0.8 .4)$; draaipunten (pivoting points) en gebieden waar de geleiding sterk vertraagd is, worden geidentificeerd door gefragmenteerde potentialen ( $P$ PV, 0.87 ). In deze patiënten zijn er geen voorkeursplaatsen woor de complexere potentialen angetroffen. De conclusie is, dat de morfologie van unipolaire electrogrammen tijdens boezemfibrilleren het optreden weergeeft van werschillende kemmerkende geleidingspatronen.

Andere studies hebben laten zien dat boezemfibrilleren met de tijd verergert. Patienten die eerst slechts zo nu en dan aanvallen van boezemfibrilleren (paroxysmaal AF) hebben, neigen chronische leiders te worden. Het boezemritme tijdens fibrilleren is gebleken sneller te worden. De vraag rijst hierdoor of activatiepatronen tijdens recent ontstaan boezemfibrilleren (acuit $A F$ ) anders $z i j n$ dan tijdens chronisch A.F. Daarom zijn in hoofdstulk 4 de activatiepatronen tijdens acuut boezemfibrilleren vergeleken met die van chronisch $A f$. Dit werd 
onderzocht in zeven geiten warin boezemfibrilleren electrisch werd opgewelt. De actrvatiepatronen werden beide keren gemeten tijdens een open hartoperatie. De earste keer terwijl er electrodes werden gemplanteerd op het hart, en de tweede keer ( 744 maanden later) wanneer het dier werd opgeofferd. Tijdens beide operaties werd de buitenkant wan het hart (epicondiwm) van de vrije wand wan de rechter boexem in kart gebrach. Tijdens de acute experimenten werd boezemfibrilleren opgewekt door een hele snelle serie electrische prikkels (burst pacing) toe te dienen. Deze episodes duren slechts enkele seconden. Chromisch boezenfibrilleren (duurt minimal 24 uur) werd opgewekt door gedurende ongeveer een maand steeds opnieuw boezemfibrilleren op te wekken; binnen aen seconde na dat een episode gestopt was, werd er opnieuw een burst toe gediend. Hierdoor neemt de duur van episodes van boezemfibrilleren toe tot dat het niet meer uit zichzelf stopt en dus chronisch is. De activatiepatronen in geiten blikken sterk vergelijkbaar met die in mensen. In vergelliking met acuut boezemfibrilleren, is het boezemritme tijdens chronisch af sneller geworden. De activatiepatronen zijn complexer (van type r naar type ur Af), en het aantal complexere potentialen (lange dubbelvoudige- en gefragmenteerde potentialen) neemt nawenant toe. De kortste tijdspanne, die werd gemeten tussen twee openvolgende slagen (interval), neemt af. Dit kortste interval benadert de refractaire periode. Verschillen in geleidingssnelheden tussen acuut en chronisch boezemfibrilleren lijken veroorzakt te worden, door een normal gezonde (fysiologische) toename van lokale geleidingsstoornissen, en normale aanpassing aan de frequentie van het fibrilleren (rate adaptation). De geleidingseigenschappen van de boezems lijken dus niet te zijn veranderd. Echter, wanneer de boezems opgerekt zijn (dilatatie), zou de geleidingssnelheid overschat kunnen worden, en dus zou de geleiding in de boezems in feite verminderd kumnen zijn. Dit hebben we niet gecontroleend. De geschatte lengte van het pad (pathlength), dat en golfje moet afleggen voordat het kan reenteren, neemt af wan $10 \mathrm{~cm}$ tijdens acuut $A$, tot $4 \mathrm{~cm}$ tijdens chronisch fibrilleren. De conclusie is, dat de toenemende stabiliteit van boezemfibrilleren deels word verklaand door een groter antal golfics. Hierdoor verkleind namelijk de kans dat alle golfjes tegelijk uitdoven. De toename in het aantal golfjes word mogelijk gemakt, doordat de circuit grootte (pathlength) afneemt. Andete factoren die een rol zouden kunnen spelen worden besproken.

In het laatste hoofdstuk van dit proefschrift (hoofdstuk 5) wordt de mogelijke rol besproken van de drie verschillende mechanismen die in de literatuur worden 
geopperd (multipele golfjes, een roterende "moeder"golf, en abnomale foci). Wr word geconcludeerd dat de experimenten, die in het kader van dit proelschrift zijn uitgevoerd, overeenkomen met de multipele-golfjes-theorie, vooral tijdens types al en II fibrilleren waar multipele golfes immers zichtbaar zijn in de actiwatiekaarten. Het blijft echter mogelijk, dat tijdens type I AF een enkele "moeder"golf de ritmestoornis kan doen woortbestaan, Er word geopperd dat het mechanisme van boezemfibrilleren bestat uit een wijd spectrum van reenterende circuits van variabele grootte. De omvang van reentry neemt dan geleidelijk toe, in samenhang met het aantal golfjes, als men gaat van boezemflutter (een sneile man regelmatige ritmestoornis in de boezems), via paroxysmaal af, naar chronisch fibrilleren. De gegevens die gepresenteerd zijn in dit proefschrift kunnen geen rol onderschrijven voor een focal mechanisme. Toch zou spontaan optredend boezemfibrilleren in patiënten met zieke boezens, een focale oorzaatk kunnen hebben. Tenslotte worden enkele mogelijkheden voor de behandeling van boezemfibrilleren besproken.

In conclusie; tijdens electrisch opgewekt boezemfibrilleren in een groep jonge mensen, worden verschillende gradaties in de complexiteit van de boezemactiviteit gevonden. De mate van complexiteit word gekenschetst door classificatie van verschillende typen boezemfibrilleren. Gaande van type I naar type III, versnelt het boezemritme en wordt het onregelmatiger, de incidentie van reentry neemt toe, en er worden meer complexere potentialen gemeten. De morfologie van lokale unipolaire electrogrammen voorspelt het optreden van lokale geleidingsstoornissen. Vergelijking van electrisch opgewekt acuut en chronisch boezemfibrilleren in geiten, laat zien dat het boezemritme versnelt, en dat de boezemactiviteit complexer wordt. Geleiding blijft normal voor de frequentie van het boezemfibrilleren, maar dit beeld zou overschat kunnen worden door dilatatie van de boezems. De circuigrootte is tijdens chronisch boezemfibrilleren afgenomen. Hierdoor passen er meer golfjes tegelijkertijd in de boezems en wordt de kans op het uitdoven van boezenfibrilleren verkleind. Deze bevindingen zouden werder inzicht kunnen geven in het mechanisme van boezemfibrilleren. Het werschil tussen flutter-fibrilleren, en grof en fijn fibrilleren zou gebaseerd kunnen zijn op variabele formaten van reenterende circuits. Toch moet men niet vergeten dat patiënten vaak een onderliggende zickte hebben die hun boezemfibrilleren veroorzakt. Bij hen zouden wel cens andere mechanismen een rol kunnen spelen dan in deze experimenten. Het medische belang van de graad yan complexiteit is dus nog onzeker en verder onderzoek is noodzakelijk. 

Appendix | Nawoord | 


\section{Nawoord}

In het derde jaar van mijn doctoraal studie, werd ik tijdens een wetenschaps-stage besmet met het research-virus. Ik had er al een genetische vatbaarheid voor, maar werd er in de vakgroep fysiologie volledig door gegrepen. lk genoot van de experimenten, van het gepuzzel, van de samenwerking tussen mensen uit velerlei disciplines en landen. De combinatie van doen èn denken beviel me uitermate goed, maar het allermooiste vond ik de intrinsieke motivatie, die uit het onderzoek voortvloeit. Iedere hypothese, die je onderzoekt levert antwoorden, maar ook nieuwe vragen op, en ook die wil je weer kunnen beantwoorden. Totaal naief heb ik me erop gestort, en ik was dolgelukkig met de mogelijkheid om er na mijn doctoraal als promovendus mee door te gaan.

De naiviteit is me weleens duur komen te staan, maar het plezier in het onderzoek werd alleen maar sterker. Ik ontdekte steeds nieuwe uitdagingen. Steeds dieper graven, maar het overzicht behouden. Enorme creatieve vrijheid, maar ook dwang om kritisch te blijven. Het genoegen dat je blijft leren, met de frustratie dat je weinig zèker weet. De stimulerende werking van overleg met collega's, wat dan weer leidt tot het verlammende besef, dat alle verschillende observaties maar moeilijk in èèn concept passen. Savoir pour savoir, maar ook voor het hoger doel; behandeling van patiënten. Diepe dalen en hoge toppen. Deze dingen behelzen voor mij de wereld van de wetenschap.

En dan is er nu eindelijk mijn proefschrift. Promoveren doe je niet in je eentje. Collega's en zij, die me lief zijn, zijn onontbeerlijk geweest voor de totstandkoming hiervan. Dit boekje stelt mij in de gelegenheid in het openbaar dank te zeggen aan hen, die in bijzondere mate aan mijn vorming hebben bijgedragen. Tot mijn spijt ben ik gedwongen mij daarbij te beperken.

Allereerst mijn promotor Prof Dr. M.A. Allessie. Beste (grote) Maurits, voor sommige dingen kan ik je moeilijk bedanken, maar andere dingen zijn makkelijker onder woorden te brengen. Ik heb veel wan je geleerd. Met jou te werken is een intellectuele ervaring. Je enthousiasme voor onderzoek is altijd aanstekelijk voor me geweest. Je weet me steeds te prikkelen om dieper te graven, om alles wat in de data verborgen zit, eruit te halen. Je beruchte kritische houding, perfectionisme, onstuitbare ideeënstroom, en zeer persoonlijke opvattingen over schrijven en presenteren, zijn fundamenten van dit proefschrift. le hebt me regelmatig tot wanhoop gedreven, maar je draagt ook de ingrediënten aan voor goede wetenschap. Bedankt hiervoor.

Dr. C.J.H.J. Kirchhof. Beste Charles/Charley, je hebt me het dagelijks leven van een 
wetenschapper bijgebracht. Het leek me in eerste instantie een wat deplorabel vooruitzicht, want eten, slapen en wrije tijd kwamen er nauwelijks in woor. Het ging zelfs zó ver, dat je net als de honden waarmee je experimenteerde, slechts eenmaal per dag een maaltijd at. Maar ik leerde al snel van je, dat het léuk is om je in je experimenten en analyses te storten, dat het pas ècht interessant wordt als je goed in je literatuur zit en, dat de schoonheid van onderzoek ' $m$ zit in perfectie.

Dr. M.C.E.F. Wijffels. Beste (kleine) Maurits, ik heb je altijd bewonderd om de goedgehumeurde gedrevenheid waarmee je je onderzoek deed. Je was altijd bereid tot hulp, meedenken, en een grapje. Zonder jou had ik de analyses in de geiten niet kunnen doen. Je bracht een prettige sfeer in het lab en leverde prachtigg onderzoek. Bedankt.

De leden van de beoordelingscommissie, Prof Dr. H.J.. Wellens, Prof Dr. H.J.G.M. Crijns, Prof Dr. H.J. Jongsma, Prof Dr. R.S. Reneman en Dr. J.L.R.M. Smeets. Bedankt voor de bereidheid, het manuscript kritisch door te lezen en om op de verdediging van mijn proefschrift aanwezig te zijn. Prof Wellens, ik wil u ook bedanken, voor mogelijkheid om experimenten in patiënten uit te voeren en uw waardevolle bijdragen aan de manuscripten wan hoofdstuk 2 en 3. Prof Crijns, beste Harry, ik wil jou ook bedanken, voor de aangename ontmoetingen die we op diverse congressen hebben gehad, en je stimulerende kritiek. Prof Jongsma, gezien uw indrukwekkende wetenschappelijk oeuvre, waardeer ik uw oordeel over mijn proefschrift zeer. Prof Reneman, beste Rob, dankzij u heb ik een cruciale drempel kunnen nemen. Hierwoor mijn onuitsprekelijke dank. Dr. Smeets, beste Joep, zonder jouw werk met de patiënten, had ik dit onderzoek niet kunnen doen. Ook hiervoor bedankt.

Paranimfen spelen een speciale rol. Gezien het onderwerp van mijn onderzoek mag het dan ook niet geheel toevallig heten dat ik twee "boezem"-vrienden heb gevraagd. Gert, in het dagelijks leven sta je me bij, sta je vierkant achter me, en sta je je mannetje, als ik dat nodig heb. Ik ben dan ook blij dat je op deze dag nàst me wilt staan. Rik, je bent al jaren een fantastische vriend en ik bewonder je doorzettingsvermogen; je hebt vorm weten te geven aan cen vak dat slechts uit ideeèn leek te bestaan. He onvermoeibare en soms onverklarbare inzet hiervoor, en het resultaat dat je bereikt hebt, heeft me meer dan eens geinspireerd om door te gaan. Bedankt voor de steun en je onwankelbaar vertrouwen in mijn capaciteiten. Ik ben blij dat ook jij aan mijn zijde wilt stan, maar een oranje of gebloemd rokkostuum zal toch niet kunnen.

Dr. L.V.A. Boersma, beste Lucas. je was mijn mede-promovendus en mijn huisgenoot 
(wat op en gegeven moment nogal wat verwarning teweegbracht op het lab). De discussies ower de dagelikse research troubles het ping-pongen, de golfspelletjes en de analyses van de nerd-outfits van de andere wetenschappers, leverden voor mij een wezenlijke bijdrage tot het met plezier uitvoeren van m'n onderzoek. Bedankt.

Dr. J. Brugada, dear Jep. Your tremendous energy to do research and to write has been a great example to me. I also aprecciated your scientific opinions which served as an interesting counterweight to the things I learned from (big) Maurits. I miss your bullis-kick coffee, and the good fun you always brought with you. Thank you.

Z.A. Zetelaki, dear Zoltan. You were a great roommate; while you were here, every time that If felt that things were going wrong in my research, you always gave me the idea that it was not so bad after all. 1 also miss Orsi's goulash and the fun we had. Thank you.

Dr. M.). Reiter, dear Michael. I'll never forget the time when I was a student and you were a visiting senior investigator. You came to my room to get coffee and were fuming, about everything that was going wrong in our lab. After I listened to your complaints for a while, I naively concluded: "You're doing an experiment. It's normal that everything goes wrong, so what's the problem?' Strange enough, you didn't take this as an insult, but instead became a perfect friend. You've helped me in many ways, of which the revisions of Chapter 2 in the laundromat, are one of the most remarkable. We have spend many great times together. I hope that we will continue on this foot in the future, because to me it is the perfect combination between exciting scientific contact and warm personal friendship. Thank you.

R. Dorland, beste Rick. Bedankt voor de ceuwige bereidheid om files en andere details van de geiten voor me uit te zoeken, en je droge humor. Ik zal iedere keer dat ik een geit zic keutelen weer denken an jouw "roos van vlees".

Dr. F. Mast, beste Frans. Bedankt woor het vele werk op de achtergrond en de rust die je weet over te brengen. Je me thebt meer dan eens (onbewust) van morele steun woorzlen.

J. Hollen en F: Schmitz, beste Jan en Frits. Ik zou jullhe te kort doen als ik jullie apart zou noemen omdat jullie ontosmakelijk samen de technische en komische assistentie verlenen die ik zo vaak gebruikt en misbruikt heb. Zonder jullie is ar op het lab weinig mogelijk. Bedankt.

Dr. Lis van der Zee, beste Lucie. Bedankt woor de vele koppen koffie, maar vooral voor de onontbeerlijke uitlaatklep. 
Dr. M.J.P. Kilian, beste Matthijs Je pessimisme over de oplosbararheid van experimentele problemen, in combinatie met je euwige enthousiasme over de experimentele resultaten, gaf me regelmatig nieuwe impulsen. Bedank hiervoor,

De dames van het secretariaat, met name Claire Bollen. Bedankt voor het onuitputtelijk regelen van afspraken met Maurits, beantwoorden wan wragen en toeschuiven van schrijmaterialen.

The very welcome guests; Flavia Ravelli, Javier Chorro, Kai Haberl, John Power, Clifford Garratt, Jean-Claude Deharo, Olivier Piot and Bodo. Thank you for the pleasant company in the lab and during dinners, drinks, and congresses. Each of you supplied me with interesting scientific input from at different angle and with a lot of fur.

De huidige generatie; Matthias Duytschaever, Peter Danse, lannie Ausma en Sabine Eysbouts. Julle houden me bij de tijd. Het is een genoegen om samen met jullie nog een project te doen, en jullie enthousiasme is aanstekelijk. Bedankt.

De andere colllegae van fysiologie. Bedankt voor het eindeloos vragen hoe het met mij en min proefschrift gat. Jullie weten het steeds weer positief te brengen.

De chaos-mannen; Prof Dr. I de Goede en Dr. B Hoekstra, beste Jap en Bart. Bedankt. voor de intensieve wetenschappelijke discussies die zich uitstrekten nar boeinde gesprekken in de vrije tijd.

And last but not least among the scientific collegues; Dr. M. Franz, dear Michael. Thank you for the fascinating scientific and personal discussions which were often accompanied by most original activities. I'm looking forward to working with you in the near future.

Ik ben ook dank verschuldigd aan Melianthe Wouters, Henk Lenting, en de anderen van Bureau Lenting en Terlingen. Melianthe, je hebt hier een prachtig professioneel boekje van gemaakt. Ik weet dat je er vele vrije uren in hebt gestoken, en ik heb genoten van je ideeën. Bedankt. Henk, ik vind het onvoorstelbar dat je direct bereid was om mij, als vriendin van een oud ulerling, te helpen on dit proefschrift vorm te geven. Jij en je team hebben zich belangeloos van een, voor mij moeilijk te realiseren, taak gekweten. Ik wind het geweldig. Bedankt.

Hier will ik ook enkele woorden van dank richten an mensen die me lief zijn: Mijn ouders, lieve pa en Ma. Iullie stimulans voor persoonlijke ontwikkeling zou ik ieder kind gunnen. Jullie levenskunst is uniek, en dankzij jullie ben ik wie ik ben. Samen èn los van elkaar hebben jullie een belangrijke bijdrage an het feit dat ik. nu promoveer. Lieve Wil, je hebt me altijd geleerd dat eigenwijsheid cen goede 
eigenschap is. Je hebt dat misschien een paar keer te valk gezegd, maar het heeft me geholpen om steeds datgene te doen warwan ik voel dat het goed is. Je feilloze timing om te bellen als iets me dwarszit, helpt me altijd weer om vertrouwen in mezalf te vinden, en jouw opwatting dat wetenschap het leukste vak is blikt ook woor mij te gelden. Bedanlkt. Lieve Ine, je "bewust naïwe" instelling heeft me geleerd om steeds weer het goede van iedereen en iedere situatie te zoeken. Je fonomenale vermogen om te luisteren en daarna cen rustige analyse te geven is onontbeerlijk voor me geweest. Bedankt.

M"n "reserve"-ouders; Liewe Jaap en Ineke. Vol interesse, steun en practische tips staan jullie altijd klaar. laap, je "inside" information over het vak geneeskunde heeft me al als klein pukkie, dewens doen uiten om later met jou te werken. Nu het "later" is, heb je me regelmatig een hart onder de riem gestoken om die wens door te zetten. Bedankt. Ineke, je inzet voor ons is al heel bijzonder, maar je proofreading van mijn proefschrift is weergaloos. Bedankt dat je er zo veel tijd in nebt willen steken. Nu kunnen we gaan genieten, en ilk vertrouw er op dat je weer de nodige foto's zult maken om het vast te leggen..

Het "sextet"; Lieve Josje, Olleke, Gerrie, Marita en Inge. Jullie samen bieden me steeds weer een geweldige vriendschap en ieder apart vullen jullie mij aan, met net die capaciterten die ik ontbeer. Jullie zijn meer dan eens de confrontatie met me aan gegaan als ik vast dreigde te lopen, en hebben me zo enorm geholpen. Bedankt. Olleke cn Gerrie verdienen een extra vermelding. Olleke, bedankt voor je ideeen en de hulp bij het regelen van de layout. Gerrie, dankzij jou zie ik er vandaag prachtig uit.

En dan, maar eigenlijk het belangrijkste; Lieve Gert. Mijn dank voor jou is onuitsprekelijk. Zonder jou zou ik nog steeds an het ploeteren zijn. Je hebt me eindeloos aan gehoord, en me gedwongen om te praten als ik niet meer wou of kon. Dat was voor jou niet makkelijk, mar dat nam je op de koop toe. Je hebt me wak geholpen, door je heldere analyses wan een wetenschappelijk detail warmee ik worstelde. Ze zeggen weleens dat promoveren een aanslag is op je relatie, maar jij hebt mij altijd het gevoel gegeven dat het onze band alleen matr versterkt. Ik draag dit proefschrift dan ook an jou op.

Er zijn vele andere vrienden en collega's die ik niet genoemd heb. Ook jullie wil ik bedanken voor de steun en het plezier die ik heb mogen ontvangen. 
Appendix | Curriculum Vitae | 


\section{Curriculum Vitae}

\begin{tabular}{|c|c|}
\hline 12 Juni 1967 & geboren te Groningen \\
\hline $1979-1985$ & wwo-s, St. Maartenscollege te Groningen \\
\hline $1985-1990$ & Doctoraal Studie Geneeskunde, Unwersiteit Maastricht \\
\hline $1990-1993$ & Medewerker Onderzoeker, Fysiologie, Universiteit Maastricht \\
\hline $1994-1997$ & $2^{c}$ fase Studie Geneeskunde \\
\hline 1997-heden & Afronding Proefschrift \\
\hline begin 1999 & Artsexamen \\
\hline
\end{tabular}

\section{Lijst van Publicaties}

\section{Papers}

KKonings, M Allessie: Mapping of atrial fibrillation in man. New Trends Arrhyth $1991: 7: 81-84$

I Brugada, L Boersma, C Kirchhof, Z Zetelaki, $\mathbb{H}$ Abdollah, K Konings, M Allessie: Sustained Monomorphic Ventricular Tachycardia: A single electrocardiographic expression of different patterns of reentry. PACE 1991;14:1943-1946

CJHJ Kirchhof, FJ Chorro, GI Scheffer, ] Brugada, K Konings, Z Zetelaki, M Allessie: Regional entraimment of atrial fibrillation studied by high-resolution mapping in open-chest dogs. Circulation 1993;88:736-749

KTS Konings, CHJH Kirchhof, JLRM Smeets, HIJ Wellens, OC Penn, MA Allessie: High-density mapping of electrically induced atrial fibrillation in humans. Circulation 1994;89:1665-168:0

MA Allessie, K Konings, CHJH Kirchhof, M Wijfels: Electrophysiologic mechanisms of perpetuation of atrial fibrillation. An / Cardiol 1995;77:10A-23A

MA Alessie, CIHI Kirchhof, KTS Konings: Unraveling the electrical mysteries of atrial fibrillation. Eur Heart $\int 1996 ; 17: 2-9$

KTS Konings, CHJH Kirchhof, ILRM Smeets, OC Penn, HJJ Wellens, MA Allessie: Configurations of unipolar electrograms durung atrial fibrillation in humans. Circulation 1997;95:1231-1241 
IM Smith HT Sih, KTS Konings, S Swiryn, GW Botterton, A Sahakian, V Adamov, MA Allessie: Spatial organization of atrial fibrillation in humans: a robust and readily quantifiable feature. 1998 ; to be published

KTS Konings, MCEF Wijffels, R Dorland, IF Mast, MA Allessie: Mapping during acute and chronic atrial fibrillation in the goat. 1999; to be published

\section{Chapters}

MA Allessie, K Konings, C Kirchhof: Mapping of atrial fibrillation. In: SB Ollson, MA Allessie, RWF Campbell (eds): Arrial fibrillation: Mechanisns and therapeutic strategies. Armonk, New York: Futura Publishing Company, 1994:37-49

MA Allessie, CHJH Kirchhof, KTS Konings, JLRM Smeets, OCKM Penn, HJI Wellens: Mapping and pacing of atrial fibrillation. In: AJ Camm, FW Lindemans (eds): Defibrillation and radiofrequency ablation. Armonk, New York: Futura Publishing Company, 1995:1-10

M Allessie, K Konings, M Wijffels: Electrophysiological mechanisms of atrial fibrillation. In: JP DiMarco, EN Prytowsky (eds): Atrial arrhythmias: State of the arr. Armonk, New York: Futura Publishing Company, 1995:155-161

MA Allessie, KTS Konings: Mapping of atrial fibrillation in humans. In MA Allessie, M Fromer (eds): Atrial and ventricular fibrillation and defibrillation. wechanisms and device therapy. Armonk, New York: Futura Publishing Company, 1996;\#\#

\section{Abstracts}

J Brugada, L Boersma, C Kirchhof, Z Zetelaki, H Abdollah, K Konings, M Mllessie: Monomorphic ventricular tachycardia: A single electrocardiographic expression of different patterns of reentry. Eur Heart / 1991;12:85

I Brugada, L Boersma, C Kirchhof, Z Zetelaki, H Abdollah, K Konings, M Allessic: Echowawe termination of reentrant ventricular tachycardia. Eur Heart/1991;12:84

KTS Konings, CHJH Kirchhof, MA Allessie: Different degrees of complexity of excitation patterns during atrial fibrillation in man studied by high-resolution mapping. Eur Heart J 1992:13:80 
KTS Konings, CHJH Kirchhof, MA Allessie: Different degrees of complexity in excitation patterns during atrial fibrillation in man studied by high-resolution mapping. Eur f Physiol 1992;421:R39

$M$ Wijfels, $C$ Kirchhof, $K$ Konings, $M$ Allessie: Artificially maintained atrial fbrillation causes chronic atrial fibrillation in conscious goats. Eur / Physiol 1992;420:R 89

$M$ Wiffels, $C$ Kirchhof, $K$ Konings, $M$ Allessie: Artificially maintained atrial fibrillation causes chronic atrial fibrillation in conscious goats. Eur y Physiol 1992:421:R43

KTS Konings, CHJH Kirchhof, JLRM Smeets, HJJ Wellens, OC Penn, MA Allessie: Characteristics of unipolar atrial electrograms during electrically induced atrial fibrillation in man. Circulation 1994:90:1-40

K Konings, C Kirchhof, I Smeets, H Wellens, O Penn, M Allessie: Characteristics of unipolar electrograms during electrically induced atrial fibrillation in man. $J$ Physiol 1994;479:132P

KTS Konings, CHJH Kirchhof, JLRM Smeets, HJJ Wellens, OC Penn, MA Allessie: Characteristics of unipolar atrial electrograms during electrically induced atrial fibrillation in man, Eur I Physiol 1995;430:R145

MCEF Wijffels, KTS Konings, CJHI Kirchhof, R Dorland, F Mast, MA Allessie; Changes in circuit size during the development of sustained atrial fibrillation. Circulation $1995 ; 92: 1-753$

KTS Konings,MCEF Wijffels, R Dorland, F Mast, MA Allessie: High-density mapping of the right atrium during acute and chronic atrial fibrillation in the goat. 1999; to be published 
Appendix | List of References | 


\section{List of References}

1. Lews T: A lecture on the evidence of auricular fibrillation treated historically. Br Med $1912: 1: 57-60$.

2. Hoffa $M$, Ludwig $C$ : Emige neue Versuche ueber Herzbewegung. Zeitsch Rat Med $1850 ; 9: 107-144$.

3. McWilliam JA. Fibrillar contraction of the heart. J Physiol 1887;8:296-310.

4. Sommerbrodt J: Ueber allorhythmie und arhythmie des herzen und deren wrsachen. Deutsch Archiv Kin Med 1877;19:392-423.

5. Hering $\mathrm{HE}$ : Ueber die haufige kombination von kammervenepuls mit pulsus irregularis perpetuus. Deutsch Med Wochenschr 1906;32:213-215.

6. Riegel F: Veber arhythmie des herzen. Zeitschr Exper Pathol o Ther 1909;6:619-688.

7. Lewis T: Auricular fibrillation and its relationship to dinical irregularity of the heart. Heart 1909-1910;1:306-372.

8. Mackenzie I: The inception of the rhythm of the heart by the ventricle, as the cause of continuous irregularity of the heart. Brit Med / 1904; 1:529-536.

9. Hering HE: Analyse des pulsus irregularis perpetuus. Prag Med Wochenschr 1903;28:377381 .

10. Mackenzie J: The cause of heart irregularity in influenza. Br Med / 1902;2:1411-1413.

11. Kolliker A, Muller H: Nachweis der negativen schwankung des muskelstroms am natuerlich sich contrahirenden muskel. Verhandl Phys Med Gesellsch Wurzburg 1855:6:528-533.

1.2. Engelmann: Ueber das elektrische verhalten des thatigen herzens. Arhw Ges Physiol $1878 ; 17: 68-99$.

13. Sanderson: On the time-relations of the excitatory process in the ventricle of the heart of the frog. I Physiol 1879-1880;2:384-435.

14. Sanderson: On the electrical phenomena of the excitatory process in the heart of the frog and of the tortoise, as investigated photographically. I Physio/ 1883-1884;4:32\%-338.

15. Finthoven W: Ein netues galvanometer. Anw Physik 1903;4:1059-1061.

16. Einthoven W: Le telecardiogramme. Arch Lntemat Physial 1906,4:132-164.

17. Rothberger CN, Winterberg H: Vorhoflimmern und arrhythmia perpetua. Wien Klin Wochensch 1909:22:839-844.

18. Mae GK: On the multiple wavelet hypothesis of atrial fibrillation. Arch Int Pharmacodym Ther 1962;140:183-188.

19. Mackenzic I: New methods of studying affections of the heart. 1-1W. Brit Med'/ 1905;1:519$522,587-589,702-705,759-762$.

20. Cushny AR, Edmunds CW: Paroxysmal irregularity of the hent and auricular fibrillation. Am I Mad Soi 1907:133:67-77. 
21. Lewis $\mathbb{T}$. The mechanism and graphic registration of the hear beat. London: Shat \& sons ltd. 1925.

22. Lewis T, Meakins I, White PD: The excitatory process in the dog's heart. Part I. "The auricles. Phit Trans Roy Soc 1914:205:375-420.

23. Engelmann TW: Ueber den einfuss der systole auf die motorische leitung in der herzammer, mit bemerkungen zur theorie allorhythmischer herzstorungen. Archive Ges Physiol $1895-1896,62: 543-566$.

24. Winterberg $H$; Studien ueber herzflimmern. 1 . Mitreilung. Ueber die wirkung des $N$. vagus und accellerans auf das flimmern des herzens. Archiw Ge Physiol 1907:117:223-256.

25. Lewis T: Observations upon fluter and fibrillation. Part 9. -The nature of atricular fibrillation as it occurs in patients. Heart 1921;8:193-219.

26. Mayer AG: Rhythmical pulsation in scyphomedusa. Papers from the Tortugas Laboratory, Washington 1908;1:115-131.

27. Mayer AG: Nerve-conduction in Cassiopea Xamachana. Popers from the Department of Marine Biology 1917;11:1-21.

28. Mines GR: On dynamic equilibrium in the heart. J Plysiol 1913:46:349-383.

29. Mines GR: On pulsus alternans. Proc Camb Phil Soc 1913;17:34-42.

30. Mines GR: On circulating excitation in heart muscles and their possible relation to tachycardia and fibrillation. Trons Roy Soc Can 1914;8:43-52.

31. Lewis T: Observations upon flutter and Gbrillation. Parl 4. -impure thutter; theory of circus movement. Heart 1918-1920;7:293-345.

32. Garrey WE: "The nature of fibrillary contraction of the heart; its relation to tissue mass and form. Am J Physiol 1914;33:397-414.

33. Lewis T: An address on the pathology of heart function. Lancet 1914;2:883-888.

34. Lewis T, Feil HS, Stroud WD: Observations upon flutter and fibrillation. Part 2. - The nature of auricular flutter. Heart 1918-1920;7:191-246.

35. Lewis $T_{0}$ Drury AN, Bulger $H A$ : Observations upon fluter and fibrillation. Part 6 . The refractory period and rate of propagation in the auricle: their relation to block the anticular walls and to flutter. Heart 1921;8:83-135.

36. Robinson $\mathrm{GC}$. The influence of the vagus nerves on the faradized aurictes in the dogs heart. I Exp Med 1913;17:429-443.

37. Lewis $\mathrm{T}$, Feil HS, Stroud WD: Observations upon flutter and fibrillation. Part 3. Some effects of rhythrmic stimularion of the auricle. Heart 1918-1920;7:247-292.

38. Lewis T. Drury AN, lliescu CC: A demonstration of cirus movement in clinical fibrillation of the andicles. Heart 1921:3:361-379.

39. Scherf D: Studies on auricular tachycadia caused by aconitine administration. Proc Soc Exp Biol Med 1947:64:233-239. 


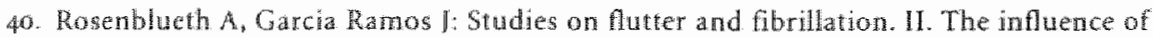
artificial obstacles on experimental auricular futter. An Heare [ 1947;33:677-684.

41. Moe GK, Abildskov IA: Atrial fbrillation as a self-sustaining arthythmias independent of focal discharge. An Heari J 1959;58:59 70.

42. Mendez $C_{8}$ Grunzit CC, Moe GK: Influence of cycle length upon refractory period of auricles, wentricles and $A V$ node in the dog. Am I Physiol 1956;184:287-289.

43. West TC, Landa JE: Minimal mass required for induction of a sustaned arrhythnia in isolated atrial segments. Am / Physiol 1962;202:232-236.

44. Moore EN, Spear JF: Natural occurrence and experimental initiation of atrial fibrillation in different animal species. In: Kulbertus HE, Olsson SB, Schlepper M: (eds). Atrial fibrillation. Molndal, Swedem: Lindgren \& Soner, 1982:33-41.

45. Hoff HE, Geddes LA: Cholinergic factor in auricular fibrillation. I Appl Plysiol 1955;8:177192.

46. Burn JH, Vaughan Williams EM, Walker JM: Effects of acetylcholine in the heart-lung preparation induding production of auricular fibrillation. / Physiol 1955;128:277-293.

47. Holland WC, Burn IH: Production of tibrillation in isolated atria of rabbit beart, Br Med I 1957:1031-1033.

48. Ravelli $F$, Allessie MA Atrial stretch decreases refractoriness and induces atrial fibrillation in the isolated rabbit heart. Circulation 1995;92:1-754 (abstract).

49. Moe GK, Rheinboldr WC, Abildskov JA: A computer model of atrial fibrillation. Am Heart) $1964 ; 67: 200-220$.

5o. Moe GK: Computer simulation of atrial fibrillation. In: Stacy RW, Waxman BD: (eds). Computers in biomedical research. New York: Academic press, 1965:217-238.

51. Moe GK. Mechanism of fibrillation in a mathematical model. 2nd Int Phamacological meeting; 1964; Prague. Perganton press. 115-118

52. Moe GK: A conceptual model of atrial fibrillation. J Electrocardiol 1968;1:145-146.

53. Alessie MA, Lammers WIEP, Bonke FM, Hollen SI: Intra-atrial reentry as a mechanism for: atrial futter induced by acetylcholine and rapid pacing in the dog. Circulation 1984;70:123. 135.

54. Allessie MA, Lammers WIEP, Bonke FlM: Total mapping of atrial excitation during acetylcholine-induced atrial fluter and fibrillation in the isolated canine heart. In: Kulbertus HE, Olsson SB, Schlepper M: (eds). Atrial fibrillation. Molndal, Sweden: Lindgren \& Soner, 1982.

55. Allessie MA, Lammers WJEP, Bonke FIM Hollen SI: Experimental evaluation of Moe's multiple wavelet hypothesis of atrial fibrillation. In: Zipes DP, Jalife I: (eds). Cardiac Electrophysiology and Antythmias. New York: Grune \& Stratton, 1985:265-275. 
56. Allessie MA, Kirchor CHH. Bonke FM: The role of the sinus node in supraventricular arrhythmias. In: Mazgalev T, Dreifus LS, Michelson EL: (eds). Electrophystology of the sinoatrial and arrioventricular nodes. New York: Alan R Liss, 1988:53-66.

57. Allessie MA, Rensma PL, Lammers WJEP, Kirchof CHH: The role of refractoriness, conduction velocity, and wavelength in initiation of atrial fibrillation in nomal conscious dogs. In: Attuel P, Coumel P, Janse M): (eds). The atrum in health and diteme. Mount Kisco. New York: Futura Publishing Company 1989:27-41.

58. Cox 1L, Canavan TE, Schuessler RB, Cain ME, Lindsay BD, Stone CM, Smith PK, Com PB, Boimeau IP: The surgical treatment of atrial fibrillation. II. Intraperative electrophysiologic mapping and description of the electrophysiologic basis of atrial futter and atrial fibrillation. I Thor Card Surg 1991:101:406-426.

59. Wang $Z_{*}$ Page PL, Nattel $S$ : Mechanism of flecainide's antiarthythmic action in experimental atrial fibrillation. Circ Res 1992;11271-287.

60. Schuessler RB, Kawanoto T, Hand DE, Mitsumo M, Bromberg BI, Cox IL, Boincau IP: Simultaneous epicardial and endocardial activation sequence mapping in the isolated canine right atrium. Circulation 1993;88:250-263.

61. Kirchiof CJH, Chorro F, Schetter GJ, Brugadá I, Konings KTS, Zetelaki Z, Allessie MA: Regional entraimment of atrial fibrillation studied by high-resolution mapping in openchest dogs. Circulation 1993;88:736-749.

62. Nadeau RA, Roberge FA, Billette $\mathrm{J}$ : Role of the simus node in the mechanism of cholinergic atrial fibrillation. Circ Res 1970;27:129-138.

63. Azuma $\mathrm{K}$, Shinmura H, Shimizu $\mathrm{K}_{\mathrm{o}}$ Iwane $\mathrm{H}$ : Significance of the sino-atrial node on mechanism of occurrence of atrial fibrillation. Jpr Heart $\int 1.972 ; 13: 84-98$

64. Kirchof $\mathrm{CIHI}$, Alessie MA: Sinus node automaticity during atrial fibrillation in isolated rablit hears. Circulation $1992 ; 86: 263-271$.

65. Hoffman BF, Rosen MR: Cellular mechanisms for cardiac arthythmias. Circ Res 1981:49:1 15.

66. Allessie MA, Rensma PL, Brugada I, Smeets ILRM, Penn O, Kirchhof CJ H] : Reentry and atrial fobrillation. Proc Kon Ned Akad Wetensch 1990;93:351-364.

67. Rothberger CJ, Winterberg $\mathrm{H}$ : Ueber wothoftimmern und vorhothattern. Eur I Physiol 1914:160:42-90.

68. Engelmann TW: Refraktaere phase und kompensatorscher tuhe in ihrer bedeurung fuer den hererhythmus. Eur / Physiol 1894-1 895;59:309-349.

69. Garrey WE: Auricular fibrillation. Physiol Rew 1924:4:215*250,

70. Hewlett AW, Wilson FN: Coarse auricular fibrillation in man. Arch Intern Med 1915,15:786788. 
7.. Nelson RM, lenson CB, Davis RW. Differential atrial arthythmias in cardiac surgical pativents. Thor Card \$urg 1969;58:581-587.

72. Wells IL, Karp RB, Kouchoukos NT, Madean WAH, James TN, Waldo AL: Characterization of atrial fibrillation in man: Studies following open heart surgery. PACE 1978;1:426-438.

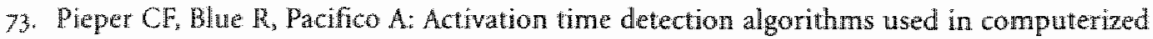
intraperative cardiac mapping: a comparison with manually determined activation times. ) Cardiovase Electrophysiol 1991;2:388-397.

74. Lammers WIEP, Schalij MJ, Kirchhof CHHJ, Allessie MA: Quantification of spatial inhomogeneity in conduction and initiation of reentrant atrial arrhythmias. Anw I Physial 1990:28:H1254-H1263.

75. Allessie MA, Hoeks APG, Schmitz GML, Reneman RS: On-line mapping system for the visualization of the electrical activation of the heart. Int J Cardiac Imag 1986;2:59-63.

76. Hoeks APG, Schnitz GML, Allessie MA. Jas H, Holken SJ, Reneman RS: Multichannel storage and display system to record the electrical actiwity of the heart. Med Biol Eng Compur 1988;26:434-438.

77. Allessie MA, Kirchhof CIHI, Scheffer GJ, Chorro FJ, Brugada J: Regional control of atrial fillyillation by rapid pacing in conscious dogs. Circulation 1991;84:1689-1697.

78. Godtfredsen ): Atrial fibrillation: course and prognosis. A follow-up study of 1212 cases. In: Kulbertus HE, Olsson SB, Schlepper M: (eds). Atrial Fibrillarion. Molndal, Swedern: Lindigren \& Soner, 1982:134-138.

79. Spach MS, Dolber PC: Relating extracellular potentials and their derivates to anisotropic propagation at a microscopic level in human cardiac muscle. Cinc Res 1986;58:356-371.

80. Kalbfleisch S1, EL-Atassi R, Calkins H, Langberg J, Morady F: Inducibility of atrial fibrillation before and after radiofrequency catheter ablation of accessory atrioventricular connections. J Cardiowasc Electrophysiol 1993;4499-503.

81. Konings KTS, Kirchhof CHH, Smeets ILRM, Wellens HIJ, Penn OC, Allessie MA: Highdensity mapping of electrically induced atrial fibrillation in humans. Circulation $1994: 89: 1665-1680$.

82. Schoels W, Restiwo M, Caraf MA, Gough WB. El-Sherif N: Circus mowement atrial flutter in canine sterile pericarditis model. Activation patterns during entrainment and termiration of single-loop reentry in wivo. Circulation 1991:83:1716-1730.

83. Shindzu A, Nozaki A, Rudy Y, Waldo AL: Multiplexing studies of effects of rapid atrial pacing on the area of slow conduction during atrial flutter in canine pericarditis model. Cipculation 1991:83:983-994.

84. Cosio FG, Arribas F, Palacios 1. Tascon 1, LopezwGil M. Fragmented ellectrograms and continuous electrical activity in atrial flutter. Am J Cardiol 1986;57:1309-1314. 
85. Cosio FG. Arribas F, Barbero IM, Kallneyer $C_{i}$ Goicolea A: Validation of double-spike electrograms as markers of conduction delay or block in atrial futter. Arm J Cardiol $1.988: 51: 775-780$.

86. Kay GN, Epstein AE, Plumb V]: The region of slow condaction in sustained ventricular tachycardia: Drect endocardial recordings and finctional characterization in humans. $\mathrm{Am}$ Coll Cordiol 1988;11:109.117.

87. Gardner PI, Unsell PC, Fenoglio H, Ir., Wit AL: Flectrophysiologic and anatomic basis for fractionated electrograms recorded from healed myocardial infarcts. Circulation $1985 ; 72: 596-611$.

88. Dillon SM, Allessie MA, Ursell PC, Wit AL: Infuences of anisotropic tissue structure on rentrant circuits in the epicardial border zone of subacute canine infarcts. Circ Res $1988 ; 63: 182-206$.

89. Schalii MI, Lammers WJEP, Rensma PL. Allessie MA: Anisotropic conduction and recntry in perfused epicardium of rabbit lett ventricle. Am J Plysiol 1992:32:H1.466-H,1478.

go. Spach M, Miller W: The discontinuous nature of propagation in normal canine curdiac muscle. Circ Res 1981;48:39-54.

91. Spach MS, Miller WT, Dolber PC, Kootsey IM, Sommer JR, et al.: The functional role of structural complexities in the propagation of depolatization in the atrium of the dog: cardiac conduction disturbances due to discontinuities of effective axial resistivity. Circ Res $1982 ; 50: 175-191$.

92. Spach MS, Dolber PC, Heidlage IF: Interaction of inhomogeneities of repolarization with anisotropic propagation in dog atria. Circ Res 1989;65:1612-1631.

93. Kadish AH, Shinnar M, Moore EN, Lewine JH, Balke CW, Spear JF: Interaction of fiber orientation and direcrion of impulse propagation with anatomic barriers in anisotropic canine myocardium. Circulation 1988;78:1478-1494.

94. Kadish A, Spear I: Identification of conduction block in cardiac muscle: In vitro observations in canine epicardium. Cardiovase Re; $1994228259-269$.

95. Spach MS, Barr RC, Serwer GA, Johnson EA, Kootsey IM: Collision of excilation waves in the dog purkinje system: Extracell ular identification. Circ Res 1971:29:499-511.

96. Wit AL, Allessie MA, Bonke FIM, Lammers WIEP, Smeets JLRM, Fenoglio II, Ir.: Electrophysiologic mapping to determine the mechanism of experimental ventriculat tachycardia initiated by premature impulses. Experimental approach and initial results demonstrating reentrant excitation. Am / Cardiol 1982:49:166m185.

97. Gerstenfeld EP, Sahakian AV, Swiryn SP: Evidence for transicnt linking of atral excitation during atrial fibrillation in humans. Cinculation 1992;86-375-382.

98. Allessie MA, Bonke FIM, Schopman FIG: Circus movement in rabbit atrial musclic as a 
mechanism of tachycardia. IIl. The "leading circle" concept: A new model of circus mowement in cardiac tissue without the inwolvement of an anatomical obstacle. Cre Res $1977 ; 41: 9-18$.

99. Feld GK, Shahandeh-Rad F: Mechanism of double potentials recorded during sustaned atral tutter in the canine right atral crush-injury model. Circulation 1992,86:628-641.

100. Puech $P$ Latour H. Grolleau R: Le nutter et ses limites, Ard Mal Coeur 1970;63:1 16-144.

10n. Joscphson ME, Horowitz LN, Spielman SR, Waxman HL, Greenspan AM: Role of catheter mapping in the preperative evaluation of ventricular tachycardia. An / Cardiol $1982 \times 49 ; 207 \% 220$

102. Fontaine G, Guiraudon GM, Frank RG): Double epicardial potentials in idiopathic ventricular tachycardia. Circulation 1976:54:11-78 (abstract).

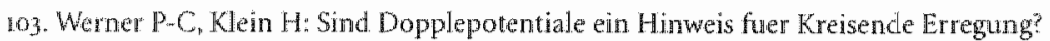
Intwatriales Katheter Mapping bei Vorhofflattern. Z Kardiol 1990;79:525-540.

104. Olshansky B, Okumura K, Henthorn RW, Waldo AL: Characterization of double potentials in human atrial futter. Studies during transient entrainment. I Am Coll Cardiol $1990 ; 15: 833-841$.

105. Olshansky B, Moreira DAR, Waldo AL: Characterization of double potentals during ventricular tachycardia: studies during transient entraimment. Circulation 1993;87:373-381. 106. Okunura K, Plumb VI, Page PL, Waldo AL: Atrial activation sequence during atrial flutter in the canine pericarditis model and its effects on the polarity of the flutter wave in the electrocardiogram. IAm Coll Cardiol 1991;17:509-518.

107. Ortiz I, Igarashi M, Gonzalez X, Johnson ND, Waldo AL: A new, reliable atrial fibrillation modet with a clinical counterpart. / Am Coll Cardiol 1993;21:183A (abstract).

108. Boinean IP, Schuessler RB, Mooney CR, Miller CB, Wylds AC, Hudson RD, Borremans IM, Brockus CW: Natural and evoked atrial futter due to circus movement in dogs. Role of abnomal atrial pathways, slow conduction, nonuniform refractory period distribution and premature beats. I Asm Coll Cardiol 1980:45:1167-1181.

rog. Durrer D, van Lier AAW, Buller I: Epicardial and intramural excitation in chronic myocartial infartion. Am Heart I 1964;68:765-776.

110. Waldo A L Kaser GA: A study of ventricular arhythmia associated with acute myocardial infartion in the canine heart. Cinculation 1973;47:1222-1228.

11. El-Sherif N, Scherlag BHF Lazzara R, Hope RR: Reentrant arrhythmias in the late myocardial infartion period. 1. Conduction characteristics in the infarct zone. Curculation $1977 ; 55 ; 686-702$.

112. Shimizu A. Nozaki A, Rudy $Y$, Waldo AL: Characterization of double potentials in a functionally determined reentrant circuit -Multiplexing studies during interruption of 
atrial Tutter in the camine pericarditis model. I Am Coll Cardiol 1993:22:2022-2032.

113. Klein H, Karp RB, Konchoukos NT, Zorm GL, Ir, James TN, Waldo AL: Intrapperative electrophysiologic mapping of the ventricles during simus thythm in partients with at previous myocardial infarction. Identification of the alectrophysiologic substrate of ventricular armythmias. Circulation $1982,668847-853$.

174. Cassidy DM, Vassalle JA, Buxton AE, Doherty W, Marchlinski FE, Josephson ME: The valut of catheter mapping during sinus whthm to localize site of origin of ventricular fachycardia. Circulation 1984;69:1103 1110.

115. Saoudi $N$, Atallah $G$, Kirkorian $G$, Touboul P: Catheter ablation of the atrial myocardum in human type I atrial tutter. Circhlation 1990;81:762-771.

116. Olshansky $B$, Okumura K, Hess PG, Waldo AL: Denonstration of an area of slow conduction in human atrial thutter. I Am Coll Cardiol 1990;16:1639-1648.

17. Fontaine G, Gwiradon FR, Tereau X. Pavie A, Cabrol C, Chomette G, Grasgogeat Y: Surgical management of ventricular tachycardia not related to myocardial ischemia. In: Josephson ME, Wellens HII: (eds). Tachycandor. Mechanism, dingnosis and meatment. Philadelpha: Hea \& Febiger, 1984:451-473.

118. Ursell PC, Gardner PI, Abala A, Fenoglio J), Jr. Wit AL: Structurat and electrophysiological changes in the epicardial border zone of canine myocadial infancts during infarct healing. Cinc Res $1985,56: 436 m 451$.

119. Brugada I. Mont L, Boersma L, Kirchhof, C., Allessie A: Differential effects of heptamol, potassium, and tetrodotoxin on reentrant ventricular tachycardia around a fixed obstacle in anisotropic myocardium. Circulation 1991;84:1307-1318.

120. Cox IL, Schuessler RB, D'Agostino HJ, Stone CM, Chang B, Cain ME, Con PB, Boneau IP: The surgical treatment of atrial fobrillation. III. Development of a definitive surgical procedure. I Thor Card Surg 1991;101:569-583.

121. Swarty IF, Pellersels G, Silvers I, Patten L, Cervantez D: A catheter-based curative approach to atral fibrillation in humans. Circhation 1994;90:1-335 (abstrat).

122. Holm M, Johansson $R$, Olsson SB, Brandt I, Luhrs $C: A$ new method for analysis of atial activation during chronic atrial fbrillation on man. IEEE Trans Bioned Evg 1996;43:198. 210 .

123. Lip GY, Tean KN, Dum FG: Treatment of atral hbrillation in a district general hospital. $\mathrm{Br}$ Heart I 1994;71:92-95.

124. Brand FN, Abbott RD, Kannel WB, Wolf PA: Characteristics and prognosis of lone anial fibrillation. 30-Year follow-up in the Framinghan study. I Am Med Assec 1025:254:34493453 .

125. Sakamoto H, Okamoto R. Imataka K, leki K, Fuji I: Prediction of early developynent of 
Chronic nourhenmatic atrial fibrillation. Jpn Hean J 1995;36:191-199.

126. Kopecky 5L, Gersh B). McGoon MD, whisnant JP, Holmes DR, Astrup DM, Frye RL. The natural history of lone atrial fibrillation: a population based study over three decades. $N$ Engly Med 1987,317:669-674.

227. Goudtredsen J: Atrial fibrillation: epidemiology, pathogenesis and natural history. In: Dalen If: (ed). Atral frbrillation and its sequelat: Am I Med, Cont Educ Sernes, 1993:5-10.

128. Takahashi $N$, Seki $A$, lmataka $K$, Finjii J: Climical features of paroxysmal atrial fibrillation: an observation of 94 patients. Jph Heart J1981:22:143-149.

129. Apert IS, Petersen P, Godtfredsen ]: Atrial fibrillation: natural history, complications and management. Am Rev Med 1988;39:41-52.

30. Wiffels MCFF, Kirchhof CHH, Dorland R, Allessie MA. Atrial fibrillation begets atrial fibrillation. A study in awake chronically instrumented conscious goats. Circulation $1995 * 92: 1954-1968$.

131. Fouchand J, Le F, Py A, Guerin F: Isolated atrial fibrillation. The risk of embolism and its prevention. Presse Med 1992;21:1117-1122.

132. Petersen P, Godtfredsen I: Atral fibrillation: a rewiew of course and prognosis. Acta Med Sand $1984 ; 216: 5-9$.

133. Mnrgatroyd $\mathrm{FD}$, Camm A): Cument concepts in atrial fibrillation. Br I Hosp Med $1993,49,546-549,552,555-557$.

134. Crozier IG, Ikram $H$, Kenealy $M$, Levy $L$ : Flecainide acetate for conversion of acute supraventricular tachycardia to sinus rhythm. Am J Cardiol 1987;59:607-609.

135. Crijus HIGM, van Wijk LM, wan Gilst WH, Kingma Hل, wan Gelder IC, Lie KI: Acute conversion of atrial fibrillation to sinus rhythm: clinical efficacy of flecainide acetate. Comparison of two regimes. Fur Heant $/ 1988 ; 9: 634-638$.

136. wan Gelder IC, Crijns HIGM, van Gilst WH, van Wijk LM, Hamer HPM, Lie KI: Efficacy and safety of flecainide acetate in the mantenance of sinus rhythm after electrical cardiowersion of chronic atral fibrillation or atrial tutter. Am J Condiol 1989;64:1317-1321.

137. wan Gelder $1 \mathrm{C}$. Crims HICM, wan Gilst WH, Verwer R, Lie KI: Prediction of uneventful cardioversion and mantenance of sinus rhythm from direct-current electrical cardioversion of chronic atral fibrillation and futter. Am / Cardiol 199 $1: 68: 41-46$.

138. Suttorp MI, Kingma JH, Lie-A-Huten L, Mast EG: Intrawenous fecainide versus verapamil for acute conversion of paroxysmal atrial fibrillation on hutter to sinus rhythm. Am I Candiol 1989;63:693-696.

139. Suttorp MI, Kingma HI, Jessurun ER, Lie-A-Huen L. van Hemel NM, Lie KI: The value of dass IC antiarthythmic drugs for acute conversion of paroxysmal atrial fibrillation or Wutter to sinus rhythm. I An Coll Cardiol 1990;16:1722-1727. 
1.40. Gold RL, Haffajee Cl, Charos G, Sloan K, Baker S, Apert IS: Amiodarone for refractory atrial fibrillation. Am I Candiol 1986;57:124-127.

14. Waris $\mathrm{E}_{\mathrm{y}}$ Kreus $\mathrm{K}$, Salokanel I: Factors infuencing persistence of sinus thythm after DC shock treatment of atrial fibrillation. Acta Med Scand 1971:189:161-166.

142. Bjerkelund $\mathrm{C}$, Orning $\mathrm{O}$ : An evaluation of DC shock treatment of atrial arrhythmias. Acta Med Scand 1968;184:481-491.

143. Morris I], Jr, Peter RH, Mchntosh HD. Electrical conversion of atrial fibrillation. Immeatiate and long-term results and selection of patients. Ann Intern Med 1966;65:216-231.

14.4. Resnekov $L$, McDonald 1 : Appraisal of electroconversion in treatment of cardiac dysrhythmias. Br Heart 1968;30:786-811.

145. Chun SH, Sager PT, Stewenson WG, Nademanee K, Middlekauff MR, Singh BN: Long-term. efficacy of aniodarone for the maintenance of normal sinus rhythm in paticnts with refractory atrial fibrillation or thutter. Am J Cardiol 1995;76:47-50.

14.6. Botterton GW, Smith JM: Quantitative assessment of the spatial organization of atrial fibrillation in the intact human heart. Circulation 1996;93:513-518.

147. Wijfels MCEF, Dorland R. Allessie MA: Pharmacological conversion of chronic atrial fibrillation by dass $1 \mathrm{~A}, 1 \mathrm{C}$ and 3 drugs in the goat. A comparison between trydroguinidine. cibenzoline, flecanaide and d-sotalol. Circulation 1996;94:1-351 (abstract).

148. Wijfels MCEF, Dorland R, Killian MJP, Mast F, Allessie MA: Effects of hydroquinidint, cibezolithe, flecanaide and $\mathrm{d}$-sotalol on atrial wavelength, circuic size and excitable gap during chronic atrial fibrillation in the goat [Medical Thesis]. Maastricht University, 1996.

149. Wijffels MCEE, van der Zee L, Dorland R, Vos MA, Allessie MA: Atrial fibrillation shortens the duration and inverses the physiological rate-adaptation of the monophasic action potential duration in the goat. Circulation 1996;94:I-556 (abstract).

150. Wujffels MCEF, Kinchhof CJHJ, Dorland R, Power ], Allessie MA: Electrical remodeling due to atrial fibrillation in chronically instrumented conscious goats. Cincularion 1997;96:3710 3720.

151. Konings KTS, Smeets LLM, Penn OC, Wellens HJJ, Allesse MA: Configuration of unpolar arrial electrograms during electrically induced atrial forillation in humans. Circukan $1997 ; 95: 1231-1241$.

152. Ausma I, Wijfels M, Thoné E, Wouters Ls Allessie M, Borgers M: Structural changen of atrial myocardium after chronic atrial fibrillation in the goat. Circulation 1996.941-593.

153. Ausma J, Wijfels $M$, wan Eys $G$, Koide $M$, Ramatkers 1 , Allessie M, Borgers M: Dedifferentiation of atrial cardiomyocytes as a result of chronic atrial fibrilation. Am I Pathol 1997; 151:985-997.

154. Borgers M, Ausma I, Wijfels M, Allessie M: Atral fibrillation in the goat: a modcl for 
chonic hibernang myacardium. Circulation 1994;90:1-407 (abstract).

355. Gaspo R, Bosch RE, Thlajic M, Nattel S. Tunctional mechanisms underlying tachycardiainduced sustamed atrial fibrilation in a chronic dog model. Circulation 1997,96:4027-4035.

556. Elwan $A$, Huang $X$, Pressler ML, Zipes D: Radiofrequency catheter ablation of the atria eliminates pacing induced sustained atrial fibrillation and reduces connexin 43 in dogs. Circulation 199796:1675-1685.

157. Morillo CA, Klein GI, Jones DL, Guiraudon CM: Chronic rapid atrial pacing: structural, functional and electrophysiologic characteristics of a new model of sustained atrial fibrillation. Circulation 1995,91:1588-1595.

158. Yue L. Feng I, Gaspo R, Li GR, Wang Z, Nattel S: Ionic remodeling underlying action potential changes in a canine model of atrial fibrillation. Citc Res 1997;81:512-525.

159. Daoud EG, Niebauer M, Bogun F, Castellani M, Chan KK, Goyal R, Harvey M, Man CK, Strickbeger SA, Morady $\mathrm{F}$ : Atrial fibrillation induced shortening of atrial refractoriness in humans. Circulation 1995:92:1 404 (abstract).

160. Tieleman RG, de Langen CDJ, van Gelder JC, De Kam PI, Grandjean I, Bel KI, Wijffels MCEF, Allessie MA, Crijns HJGM: Verapamil reduces tachycardia-induced electrical remodeling of the atria. Circulation 1997;95:1945-1953.

161. Petersen P, Kastrup I, Brinch K, Godtredsen I, Boysen G: Relation between left atrial dimension and duration of atrial fibrillation. Am / Cardiol 1987;60:382-384.

162. wan Gelder ${ }_{C} \mathrm{C}$, Crijns HIGM, wan Gilst $\mathrm{WH}_{3}$ Hamer HPM, Lie KI: Decrease of right and left atrial sizes after direct-current electrical cardioversion in chronic atrial fobrillation. Am J Cardiol 1991;67:93-95.

163. Crijns HJGM, wan Gelder IC, wan Gilst WH, Hillege H, Gosselink M, Lie KI: Serial antiarrbythmic drug treatment to maintain sinus rhythm after electrical cardioversion for chronic atrial fibrillation or atrial flutter. Am J Cardios 1991;68:335-341.

164. Sanfilippo AI, Abascall VM, Sheehan M, Oertel LB, Harrigan P. Hughes RA, Weyman AE: Anial cnlargement as a consequence of atrial hbrillation. Circulation 1990;82:792-797.

165. Shapiro EP, Efron MB, Lima S, Ouyang $\mathrm{P}$, Siu CO, Bush D: Transient atrial dysfunction after conversion of chronic atrial fibrillation to sinus rhythm. An / Cardiol 1988;62:12021207.

166. Rensma Ph. Allessice MA, Lammers WJEP, Bonke FIM, Schalij MJ: Length of excitation wave and susceptibility to reentrant atrial arhythmias in normal conscious dogs. Circ Res $1988 ; 62: 395-410$.

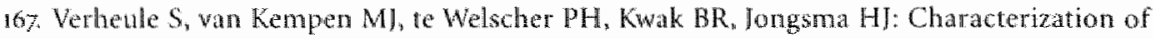
gap junction channels in adult rabbit artial and ventricular myocardium. Corro Res 1997:80:673-681. 
168. Gros D, Jarry-Guichard T, ten Velde I, de Maziete A, van Kempen MI, Davoust I, Briand IP, Moorman AF, Jongsma HI: Restricted distribution of connexin 40, a gap junctional protein, in mammahan heart. Circ Res 199474:839-851.

169. Thomas SA, Schuessler RB, Berul CI, Beardslee MA, Beyer BC, Mendelsohn ME Saffitz lt: Disparate effects of deficient expression of connexin 43 on atrial and ventricular conduction: evidence for chambar-specific molecular deteminants of conduction. Circtatation 1998:97:686-691.

170. Dahl G, Werner R: Differential closure of mRNAmduced as compared to endogenous cell to-cell channels in pairs of Xenophus oocytes. Byoptys / 1986:49:20 La (abstract).

171. Dahl G, Werner Rx Levine E: Paired oocytes: an expression system for cell-to-call channels. In: Herzberg Ex Johnson R: (eds). Gop juncions. New York: Alan R Liss, 1988:183-197.

172. Kirchtaff $S$, Nelles $\mathbb{E}$, Hagendorff $A$, Kruger $O$, Traub $O$, Willecke K: Reduced cardac conduction velocity and predisposition to arrhythmias in connexin 40 -deficient mice. Curr Biof $1998 ; 8: 299-302$.

173. Elvan $A$, Wylie $K \times$ Zipes DP: Pacing-induced chronic atrial fibrillation impairs sinus node function in dogs: electrophysiological remodeling. Chatation 1996:94:2953-2960.

174. Stambler BS, Wood MA, Ellenbogen KA: Antiarthythmic actions of intravenous ibutilide compared with procainamide during human atrial flutter and fibrillation. Electrophysiological determinants of enhanced conversion efficacy. Cinculation $1997 ; 96: 4298-4306$.

175. Tieleman RG, wan Gelder TC, Grijns HJ, de Kam PI, van den Berg MP, Haaksma J, van der Woude H], Allessie MA: Early recurrences of atral fibrillation after electrical cardioversion: a result of librillation-induced ellectrical remodeling of the atria? I Am Coll Cardiol $1998 ; 31: 167-173$.

176. Daoud EG, Knight BP, Weiss. R, Bahu M, Paladino W, Goyal R, Man KC, Strickberger SA, Morady E: Effect of verapanill and procainamide on atrial fibrillation-induced electrical remodeling in humans. Circulation 1997;96:1542-1550.

177. von Goethe JW. The sorrows of young Werther, 1774 .

178. Foster $\mathrm{E}$, Gray $\mathrm{RA}$, Jalife J: Role of the pectinate muscle structure in atrial fibrillation: a computer study. PACE $1997 ; 20: 1134$ (abstract).

179. Ong JC, Wu T-J, Karagueuzian HS, Chen P-S: Single meandering spiral wave of excitation underlies acute and chronic in situ canine atrial fibrillation. PACE 1997;20:1081 (abstrac).

180. Morillo CA, Klein GJ, Iones DL: Experimental atrial fibrillations evidence for a focal mecthanism. J An Coll Cardiol 1993,21:183A (abstract).

181. Jais P, Haissaguerre M, Shal DC, Takahashi A, Barold S, Clementy I: Effocacy and safety of linear ablation for paroxysmal atriall fibrilation in the left atrum. PACF 1997;20:1 100 
(abstract).

182. Haissaguetre M, Jais $P$, shah $D C$, Lavergme $T$, Takahashi $A$, Barold 5 , Clementy $J$ : Predominant origin of atrial panarhytmic triggers in the pulmonary weins: a distinct electrophysiologic entity. PACE 1997;20:1065 (abstrad).

183. Kuck KH, Hebe J, Schluter M, Cappato R: Irregular atrial tachycardia: complex ECG pattern caused by a single automatic focus. PACE 1997;20:1106 (abstract).

184. Wang J, Boume GW, Wang Z, Villemaire C, Talajic M, Nartel S: Comparative mechanisms of antiathythmic drug action in experimental atrial fibrillation. Circulation 1993;88:10301044.

185. Wit AL, Allessie MA: Functional models of teentry: a comparison of anisotropic and leading circle reentry. In: Waldo AL, Touboul P. (eds). Atrial futter. Armonk, New York: Futura Publishing Company; 1996.

186. Boersma UVA, Brugada 1, Kirchhof CJHI, Allessie MA: Mapping of reset of anatomic and functional teentry in anisotropic rabbit ventricular myocardium. Cinculation 1994;89:852862.

187. Allessie MA, Schalij MJ, Kirchhof ClHI, Boersma LVA, Huyberts MSP, Hollen S]: Experimental electrophysiology and arrhythmogenicity. Anisotropy and wentricular tachycardia, Eur Heart 1989;10:2-8.

188. Cabo C, Pertsow AM, Baxter WT, Davidenko JM, Gray RA, Jalife J: Wave-front curvature as a cause of slow conduction and block in isolated cardiac muscle. Circ Res 1994,75:1014-1028.

189. Waldo AL, Maclean WAH: Diagnosis and treatment of cardiac arrhythmias following open heart surgery: Emphasis on the use of epicardial wire electrodes. Mount Kisco, New York: Futura Publishing Company, 1980:85-88.

190. Kalman JM, Olgin \E, Saxon LA, Fisher WG, Lee RJ, Lesh MD: Activation and entrainment mapping defines the tricuspid annulus as the anterior barrier in typical atriall flutter. Circialation 1996;94:398-406.

191. Nakagawa H, Lazzara R, Khastgir 'T, Beckman KI, MeClelland IH, Imai S, Pitha JV, Becjker: AE, Arruda M, Gonzalez MD, et al.: Role of the tricuspid annuhs and the eustachian walve/ridge on atrial thuter. Circulation 1996;94:407-424.

192. Boineau JP: Atrial futter: a synthesis of concepts. Circulation 1985;72:249-257.

193. Frame LH, Page RL, Hoffman BH: Attial reentry around an anatomic barrier with a partially refractory excitable gap. A canine model of atrial futter. Circ Res 1986;58:495-511.

194. Guney TE, Lown B: Electrical conversion of atrial flutter to atrial fibrillation: flutter mechanism in man. Br Heart ] 1972;34:1215-1224.

195. Klein GI, Guiraudon GM, Sharma AD, Milstein S: Demonstration of macro-reentry and feasibility of operative therapy in the common type of atrial futter. Am / Cardiol 
$1986 ; 57587-591$.

196. Lewis T, Drury AN, Iliescu CC: A demonstration of citcus movement in dinical thuter of the auricles. Heart 1921;8:341-359.

197. Page PL, Plumb VI, Waldo AL: Total epicardial mapping of atrial futter in a new animal model. I Am Coll Cardiol 1983;1:716 (abstract).

198. Plumb VI, James TN, Waldo AL: Evidence that atrial flutter is due to a circus movement with an excitable gap. Circulation $1980 ; 623: 46$.

199. Rytand DA: The circus mowement (entrapped circuit wavel hypothesis and atrial flutter. Ann Intern Med 1966;65:125-159.

200. Rytand DA: Atrial lutter and the circus movement hypothesis. Cholation 1966;34:713714.

201. Schoels $W$, Gough $W B$, Restivo $M$, El-Sherif $N$ : Circus movement atrial thutter in the canine pericarditis model. Cir Res 1990;67:35 50.

202. Shoda M, Kajimoto K, Matsuda N, Unemura I, Ohnishi S, Kasanuki H: A novel mechanism of human atrial fibrillation: single macro-reentry with intra-atrial conduction block. PACE 1997;20:1065 (abstract).

203. Waldo AL, Wells JL, Phumb VI, Cooper TB, Maclean WAH: Studies of atrial flutter following open heart surgery, Ann Rev Med 1979;30:259-268.

204. Wellens HJI, Janse MJ, van Dam RT, Durrer D: Epicardial excitation of the atria in a patient with atrial flutter. Br Heart f 1971;33:233-237.

205. Wells JL, Maclean WAH, James TN, Waldo AL: Characterization of atrial futter Studies in man after open heart surgery using fixed atrial electrodes. Circulation 1979:60:665-673.

206. Anbe DT, Rubenfire $\mathrm{M}$, Drake $\mathrm{EH}$ : Conversion of atrial flutter to atrial fibrillation with carotid sinus pressure. J Electrocardiol 1969;2:377-380.

207. Bussan R, Reid ER, Scherf D: Conversion of atrial flutter into atrial fibrillation by carotid pressure. Anm Intern Med 1957;46:814-818.

208. Ortiz J, Niwano S, Abe H, Rudy Y, Jollusson NJ, Waldo AL: Mapping the conversion of atrial flutter to atrial fibrillation and atrial fibrillation wo atrial fut ter. Circ Res 1994;74:82-894.

209. Tieleman RG, de Langen CDI, van der Woude HJ, Grandjean (G, Bel KJ, Wiffels MCEF, Allessie MA, Crijns HIGM: Reduction of electrical remodeling of the atrium by verapamil. Cinculotion 1995;92:I-754 (abstract).

210.Spinale FG, Crawford FA, Hewitt KW, Carabello BA: Ventricular fallure and cellular remodeling, with chronic supraventricular tachycardia. I Thonac Cardiovase Sug $1991 ; 102: 874-882$.

2.1. Goette A, Honeycutt C, Langberg J: Ellectrical remodeling in atrial fibrillation. Time course and mechanisms. Cirulation 1996:942968-2974. 


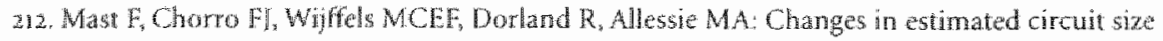
during spontancous termination of atrial forillation. Eur / Physiol 1995:430:R112 (absuract).

23. Spach MS, Kootsey IM, Sloan JD: Active modulation of electrical coupling betwean cardiac cells of the dog. A mechanism for transtent and steady state variations in conduction. velocity Cre Res 1982;51:347-362.

21. Ten Eick RE, Singer DH: Electrophysiologic properties of diseased human atrium. 1. Low diastolic potential and altered cellular response to potassium. Circ Res 1979;44:545-557.

215. Singer D, Ten Eick R: Aberrancy: Electrophysiologic aspects. Am J Cardiol 1971;28:381-401. 216. Allessie MA, Bonke FIM, Schopman FJG: Circus movement in rabbit atrial muscle as a mechanism of tachycardia. II. The role of nonumiform recovery of excitability in the occurrence of unidirectional block, as studied with multiple microelectrodes. Circ Res $1976 ; 39: 168-177$

217. Ramdat Misier AR, Opthof T, Hemel van NM, Defauw JIAM, de Bakker JMT, lanse MJ, Capelle van Fll: Increased dispersion of 'refractoriness' in patients with idiopathic paroxymal atrial fibrillation. I'Am Coll Cardiol 1992;19:1531-1535.

218. Le Hewzey 1, Boutjdir M, Gagey S, Lavergne T, Guize L: Cellular aspects of atrial wulnerability. In: Atruel $\mathrm{P}$, Olsson SB, Schepper $\mathrm{M}$ : (eds). The atrim in Health and Disease. Mount Kisco, New York: Futura Publishing Company, 1989:81-94.

219. Boutjdir M, Le Heuzey I, Lavergne T, Chauvad S, Gize L, Carpentier A, Peronneau P: Inhomogeneity of cellular refractoriness un human atrium: factor of arrhythrnia? PACE $1986: 9: 1095-1100$.

220. Kumagai $K$, Niwano $S$, Khestian $C$, Ortiz , Waldo $A L$ : Simultaneous multisite mapping during induced atrial fibrillation in the canine sterile pericarditis model - Insights into selfsustaining mechanisms. PACE 1995;18:809.

221. Kannel WB, Abbott RD, Savage DD, MCNamara PM: Epidemiologic fearures of chronic atrial fibrillation: the Framingham study. N Engl Med 1982;306:1018-1022.

2.22. Camplath $A$, Caitd FI, lackon TFM: Prevalence of abnormalities of electrocardiogram in old people. Br Heart/1974;36:1005-1011.

223. Kanned WD, Abbott RD, Sawage DD, MCNamara PM. Coronary heart disease and atrial fibrillation: the Framingham study. Am Heart / 1983;106:389-396.

224. Lie IT, Falk RH: Pathology of atral fibrillation: insights from antopsy studies. In: Falk RH, Podrid P): (eds]. Arrial fibrillaron: Mechamisms and wanagenent. New York: Rawen Press. L.t. 1992:1-18.

225.Mllessite MA, Rensma PL, Brugada J, Smeets JLRM, Penn OC, Kirchof CJHI: Pathophysiology of atrial fibrillation. In Zipes DP, Jalife I: (Cds). Cardac electrophysiology. 
From cell to bed side. Philadelphia: Samders, $1990: 548-559$.

226. Geraets DR, Kienzle $\mathrm{MG}$ : Therapy reviews. Atral Wbrillation and atrial Guter. Clin Pham $199 ; ; 12: 721-735$

227.Zarse M, Eijsbouts S, Allessie MA: Acute atrial dilatation increases heterogeneity of conduction in the langendorff-perfused rabbit heart PACE 1997201081 (abstract).

228. Spach MS, Dolber PC, Anderson PAW: Multiple tegronal diferences in cellular properties that regulate repolarization and contraction in the right atrium of adult and newborn dogs. Circ Res 1989;65:1594-1611.

229. Feinberg MS, Waggoner AD, Kater KM, Cox JL, Lindsay BD, Perez JE. Restoration of atrial function after the Maze procedure for patients with attial fibrilation. Assessment by doppler echocardiography. Circulaton 1994;90:11-285-11-292.

230. Leitch JW, Klein G], Yee R, Guiration GM: Simus node-atrioventricular node isolation: Iong term results with the "corridor" operation for atral fibrillation. I Am Coll Cardiol $1991 ; 17: 970-975$.

231. Guraudon GM, Klen GF, Guiraudon CM, Yee R: Treatment of atrial fibrillation: Preservation of silnoventricular impulse conduction (the corridor operation). In: Olsson SB, Allessie MA, Campbell RWF: (eds). Amial Fibrillaton. Mechanisws and Therapentic Strategies. Armonk, New York: Futura Publishing Company, 1994:349-371.

232. Guiraudon GM, Campbell CS, Jones DL: Combined sinoatrial node atro-ventricular node isolation: A surgical alternate to His bundle ablation in patients with atral fibrillation. Circulation 1985;72:111-20.

233. Willians IM, Ungerleider RM, Lotland GK, et al.: Left atrial isolation A new technique for the treatment of supraventricular arrhythmias. Thorac Cardowat Surg 1980;80:373-380.

23atk Kadish A, Schilling R, Peters N, Hauck J, Dawies W: Endocardial mapping of human atrial fibrillation using a novel non-contad mapping system. PACE 1997;20:1063 (abstract).

235. Le Grand $B_{*}$ Hatem $S$, Deroubaix $E$, Conenl JP. Coraboenf $E$. Depressed transient outward

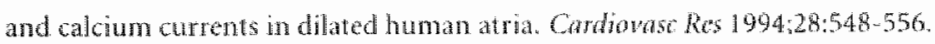

236. Fenelon G, Shepard RK, Turner DA. Manders T, Stambler BS: Evidence of a Cocal origin als the mechanism of atrial tachyourdia in dogs with wentricalar pacing induced congestive heart failure. PACE 1997;20:1095 (abstract.

237. Jais P Haissaguerre M, Shah DC, Chouari S, Gencel L, Hocini M, Clemenly I: A focal source of atial fibrillation trated by discrete radiofrequency ablation. Circulation $1997: 95: 572-576$.

238. Sallhadin $\mathrm{P}$, Bran $\mathrm{M}$, de Marneffe M, Denolin H: Management of patients with chronic atrial fibrillation. Br ] Clim Phamacol 1982;13:295-296.

239. Aberg t: Atrial fibrillation. A revew of 463 ases from philadelphia General Hospitalfrom 
1955 to 1965. Acta Med Scard 1968:184:-25 25431.

240. Furberg CD, Psaty BM, Manolio TA. Gardin JM, Smith VEx Rautaharju PM: Prevalence of atrial fibrillation in elderly subjects (The cardiovascular health study). Am / Cardiol $199474236-241$.

241. Prystowsky EN, Benson W, Furster V, Hart RG, Kay N, Myerburg RI, Naccarell: GW, Wyse G. Management of patients with atrial fibrillation. A statement for bealthcare professionals from the subcommittee on electrocardiography and electrophysiology, american heart association. Circulation 1996;93:1262-1277.

242. Grogan M, Smith HC, Gersh BJ, Wood DL: Left ventricular dysfunction due to atrial fibrillation in patients initially believed to have idiopathic dillated cardiomyopathy. Am f Cardiol 1992;69:1570-1573.

243. Wolf PA Abbott RD, Kannel WB: Atrial fibrillation as an independent risk factor for stroke: the Framingham study. Stroke 1991;22:983-988.

244. Wolf PA, Abbott RD, Kannel WB: Atrial fibrillation: a major contributor to stroke in the elderly: the Framingham study. Arch Yntern Med 1987;147:1561-1564.

245. The stroke prevention in atrial fibrillation investigators: Predictors of thromboembolism in atrial fibrillation: I. Clinical features of patients at risk. Anm Im Mod 1992;1 16:1-5.

246. Reiffel JA: Atrial fabrillation. CSE 1997:7-11.

247. Godfredsen J: Atrial fibrillation: etiology, course and prognosis. A follow-up study of 1212 cases [Medical thesis], 1975.

248. Coplen SE, Antman EM, Berlin IA, Hewitt P, Chalmers TC: Efficacy and safety of quinidine therapy for maintenance of sinus rhythm after cardiowersion. A meta-anallysis of randomized control trials. Circulation 1990:82:1106-1116.

249.Canpbell RWF: Atrial fibrillation: steering a magement course between thromboembolism and proarrhymmic risk. Eur Heart J 1995:16:28-31.

250. Kurchhof CIHT, Wiffels M. Brtigada I, Planellas J. Allessie MA: Mode of action of a new class IC drug (ORG 7797) against atrati fibrillation in conscious dogs. / Candowasc Pharmacol $1991 ; 17: 116-124$

251. Smeets JLRM. Allessie MA, Lammers WJEP, Bonke FIM, Hollen SI: The wavelength of the cardia impulse and reentmant arrhythmias in isolated rabbit atrium. "The role of heart rate, athonomic transmitters, temperature, and potassium. Circ Res 1986;58:96-108.

252. Wang I, Feng I. Nattel S: Class 111 antiarhythmic drug action in experimental atrial fibrillation. Differences in reverse use dependence and effectiveness between d-Sotalol and the new antiarthy thmic drug Ambasilide. Conchation 1994;90:2032-2040.

253. Kalman IM, Otgin JE, Karch MR, Lesh MD: Regional entrainment of atrial fibrillation in man. / Cardionase Electrophysiol 1996;7867-876. 
254. Daond EG, Pariseau B, Niebauer M, Bogun F, Goyal R, Harwey M, Man KC. Stickberger SA, Morady F: Response of type I atrial tibrillation to atrial pacing in humans. Circulation 1996;94:1036-1040.

255.Paladino W, Bahu M, Knight BP, Weiss R, Sousa J, Zivin A, Goyal R, Daoud E, Man KC, Strickberget $S A$, et al.: Falure of single and multisite high-frequency atrial pacing to terminate atrial fibrillation. Am I Cardiol 1997;80:226-227.

256. Elvan $A$, Pride HP, Eble $\mathbb{N}$, Zipes DP: Radiofrequency catheter ablation of the atria reduces inducibility and duration of atrial fibrillation in dogss. Cirulation 1995;1:2235-2244.

257 Baker BM. Smith JM, Cain ME: Nonpharmacological approaches to the treatment of atrial fibrillation and atrial futter. J Cardiowasc Electroptyssiol 1995;6:972-978.

258. Haines DE, MCRury IA: Primary atrial fibrillation ablation (PAFA) in a chronic atrial fibrillation model. Circulation 1995;92:I-265 (abstract).

259.Lurie KG, Buscemi P, Iskaos, D, Adkisson SHO, Fahy G, Shultz JI, McKnite SH Mulligan KA, Hoff JV, Benditt DG: Comparison of right atrial and peripheral Procainamide infusion levels for the treatment of atrial fibrillation. PACE 1997;20:1143 (abstract).

260. Arzbaecher $R$, Buscemi P: Pharmacologic atrial defibrillator: initial experimental studies with rapid intra-atrial infusion of procainamide. PACE 1997;20:1093 (abstract).

261. Cox JL, Boineau JP, Schuessler RB, Ferguson TB, Lindsay BD, Cain ME, Corr PB, Kater KM, Lappas DG: A review of surgery for atrial fibrillation. /Cardiovasc Electrophysiol 1991;2:541561.

262. Sayers DL: Gaudy Night. The Albatros Modern Continental Library. Leiden, NL: Sijthoff"s Uitgevers Maatschappij, 1947; volume 364. 